\title{
Property testing and parameter estimation
}

\author{
Henrique Stagni \\ TESE APRESENTADA \\ AO \\ Instituto de Matemática e Estatística \\ DA \\ Universidade De SÃo Paulo \\ PARA \\ OBTENÇÃO DO TÍTULO \\ $\mathrm{DE}$ \\ DOUTOR EM CIÊNCIAS \\ Programa: Ciência da Computação \\ Orientador: Yoshiharu Kohayakawa
}

Durante o desenvolvimento deste trabalho o autor recebeu auxílio financeiro do CNPq (141970/2015-4) e da FAPESP (2015/15986-4, 2017/02263-0).

O presente trabalho foi realizado com apoio da Coordenação de Aperfeiçoamento de Pessoal de Nível Superior - Brasil (CAPES) - Código de Financiamento 001.

São Paulo, novembro de 2020 


\section{Property testing and parameter estimation}

Esta tese contém as correções e alterações sugeridas pela Comissão Julgadora durante a defesa realizada por Henrique Stagni em 20/11/2020.

O original encontra-se disponível no Instituto de Matemática e Estatística da Universidade de São Paulo.

Comissão Julgadora:

- Prof. Dr. Yoshiharu Kohayakawa (orientador) - IME-USP

- Prof. Dr. Guilherme Oliveira Mota - IME-USP

- Prof. Dr. Tássio Naia dos Santos - Externo

- Prof. Dr. Carlos Hoppen - UFRGS

- Prof. Dr. Marcos Abraham Kiwi Krauskopf - UCH 


\section{Abstract}

STAGNI, H. Property testing and parameter estimation 2020. 88 f. Thesis (Doctorate) Instituto de Matemática e Estatística, Universidade de São Paulo, São Paulo, 2020.

A graph property $\mathcal{P}$ is said to be testable with sample complexity $q(\varepsilon)$ if, for every $\varepsilon>0$, there is a randomized decision algorithm that distinguishes objects satisfying $\mathcal{P}$ from graphs " $\varepsilon$-far" from satisfying $\mathcal{P}$, after inspecting a sample of size at most $q(\varepsilon)$ of the input graph $G$ (in particular, the sample size does not depend on $|V(G)|$ ). Although the set of testable graph properties is now well understood [AFNS09, $\mathrm{BCL}^{+} 08$ ], results for general properties $\mathcal{P}$ tipically rely on variants of Szemerédi's regularity lemma [Sze78], giving tower-type upper bounds for the sample complexity $q(\varepsilon)$. Therefore, current research in the area is focused on obtaining better bounds for the sample complexity required to test specific properties $\mathcal{P}$.

A (normalized) graph parameter $f$ is said to be estimable with sample complexity $q(\varepsilon)$ if, for every $\varepsilon>0$, there is a randomized algorithm that estimates the parameter $f(G)$ up to an additive error of $\varepsilon$, after inspecting a sample of size at most $q(\varepsilon)$ of the input $G$. If the graph parameter being estimated is the distance $\mathbf{d}_{\mathcal{P}}$ to a graph property $\mathcal{P}$, Fischer and Newman [FN07] proved that $\mathbf{d}_{\mathcal{P}}$ is estimable for every testable $\mathcal{P}$, but their proof provides a tower-type upper bound for estimating $\mathbf{d}_{\mathcal{P}}$, even if $\mathcal{P}$ can be efficiently testable.

This thesis focuses on getting better upper bounds for the sample complexity required to estimate certain parameters and test certain properties.

Our first contribution states that one can test the property of having a partition of size $k$ with any given prescribed pairwise densities with a sample complexity polynomial in $\varepsilon^{-1}$ and $k$. This result, which improves upon a previous (exponential in $k$ ) bound given in [GGR98], is an important tool for achieving our other contributions.

Our main contribution shows that if a hereditary property $\mathcal{P}$ is testable with sample complexity $q(\varepsilon)$, then distance $\mathbf{d}_{\mathcal{P}}$ is estimable with sample complexity at most exponential in $q(\varepsilon)$. In particular, for hereditary properties $\mathcal{P}$ known to be be efficiently testable, our method provides much better bounds than the ones relying on Szemerédi's regularity lemma. Our techniques also allow one to get more reasonable bounds for estimating other graph parameters.

We also prove negative results about testing graph properties described by linear constraints of subgraph densities, which were considered by Goldreich and Shinkar in [GS16]. We conclude this thesis by proving bounds for the complexity of testing that every hereditary property of configurations of points in the plane is testable.

Keywords: property testing, parameter estimation, edit distance, speed of subgraph classes, monotone properties, hereditary properties, regularity lemmas, order types, combinatorial types, Erdős-Hajnal property 


\section{Resumo}

STAGNI, H. Teste de propriedades e estimação de parâmetros 2020. 88 f. Tese (Doutorado) - Instituto de Matemática e Estatística, Universidade de São Paulo, São Paulo, 2020.

Uma propriedade $\mathcal{P}$ de grafos é testável com complexidade amostral $q(\varepsilon)$ se, para todo $\varepsilon>0$, existe um algoritmo aleatorizado que distingue grafos que satisfazem $\mathcal{P}$ de grafos " $\varepsilon$-longe" de a satisfazer $\mathcal{P}$, após inspecionar uma amostra de tamanho no máximo $q(\varepsilon)$ do grafo de entrada $G$ (em particular, o tamanho da amostra independe de $|V(G)|)$. Apesar do conjunto de propriedades testáveis ter sido completamente caracterizado [AFNS09, $\mathrm{BCL}^{+} 08$ ], resultados gerais sobre testabilidade costumam se basear em variantes do lema da regularidade de Szemerédi e dão cotas superiores do tipo torre de exponenciais para $q(\varepsilon)$. Portanto, a pesquisa na área tem se concentrado em obter melhores cotas para a complexidade amostral para se testar certas propriedades $\mathcal{P}$.

Um parâmetro (normalizado) de grafo $f$ é estimável com complexidade amostral $q(\varepsilon)$ se, para todo $\varepsilon>0$, existe um algoritmo aleatorizado que computa $f(G)$, a menos de um erro aditivo de $\varepsilon$, após inspecionar uma amostra de tamanho no máximo $q(\varepsilon)$ do grafo de entrada $G$. Se o parâmetro em questão é a distância $\mathbf{d}_{\mathcal{P}}$ a uma propriedade $\mathcal{P}$, Fischer e Newman [FN07] provaram que $\mathbf{d}_{\mathcal{P}}$ é estimável para toda propriedade testável $\mathcal{P}$. Contudo, o método deles fornece uma cota do tipo torre, mesmo para propriedades $\mathcal{P}$ que podem ser eficientemente testadas.

O objetivo desta tese é fornecer melhores cotas superiores para a complexidade amostral para estimar certos parâmetros e testar certas propriedades.

Nossa primeira contribuição afere que é possível testar se um grafo admite uma partição de tamanho $k$ com densidades pré-especificadas entre pares de partes com complexidade amostral polinomial em $\varepsilon$ e $k$. Esse resultado, que representa uma melhora em relação à cota exponencial em $k$ obtida em [GGR98], é usado como ferramenta em nossas outras contribuições.

Nossa principal contribuição afere que se uma propriedade hereditária $\mathcal{P}$ é testável com complexidade amostral $q$, então $\mathbf{d}_{\mathcal{P}}$ é estimável com complexidade amostral apenas exponencial em $q$. Em particular, para propriedades hereditárias $\mathcal{P}$ que podem ser eficientemente testáveis, nosso método fornece cotas melhores do que as baseadas no lema da regularidade de Szemerédi. As técnicas empregadas também nos permitem obter cotas mais razoáveis para estimar outros parâmetros de grafos.

Provamos também resultados negativos a respeito de propriedades consideradas por Goldreich e Shinkar [GS16], descritas por restrições lineares das densidades de subgrafos. Concluímos a tese mostrando que propriedades hereditárias de configurações de pontos no plano são testáveis.

Palavras-chave: teste de propriedades, estimação de parâmetros, distância de edição, velocidade de classes de grafos, propriedades monótonas, propriedades hereditárias, lemas de regularidade, o-tipos, tipos combinatórios, propriedade de Erdős e Hajnal 


\section{Contents}

1 Introduction $\quad 1$

2 Preliminaries $\quad 5$

2.1 Inequalities for random variables $\ldots \ldots \ldots \ldots \ldots \ldots$

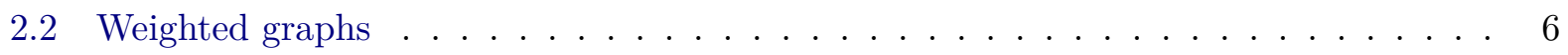

2.3 Simple graphs . . . . . . . . . . . . . . . . . . . . . . . . 7

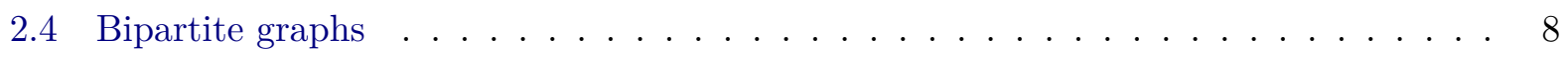

2.5 Graph properties . . . . . . . . . . . . . . . . . 8

2.6 Graph norm and distance . . . . . . . . . . . . . . . . . . 9

2.7 Homomorphisms of graphs . . . . . . . . . . . . . . . . . . . . 11

2.7 .1 Homomorphism density for graphs . . . . . . . . . . . . . 11

2.7.2 Homomorphism density for bipartite graphs . . . . . . . . . . . . . . . 12

2.8 Positive correlation between homomorphism densities . . . . . . . . . . . . . 13

3 Partitions, regularity and removal lemmas $\quad 17$

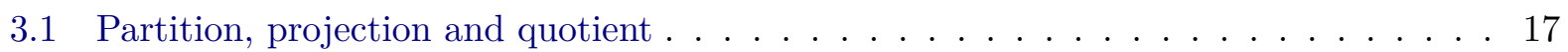

3.2 Regular and quad-regular pair . . . . . . . . . . . . . . . . . . . . 19

3.3 Frieze-Kannan regularity . . . . . . . . . . . . . . . . . . . . 21

3.4 Szemerédi regularity and variants . . . . . . . . . . . . . . . 24

3.5 Counting Lemmas . . . . . . . . . . . . . . . . . . . . . . . 25

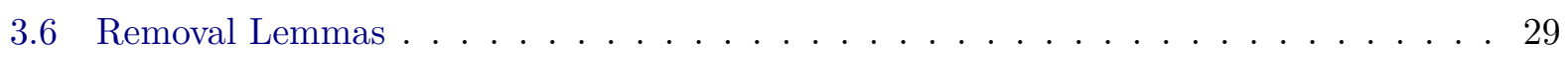

3.6.1 Removal Lemma for a single graph . . . . . . . . . . . . . . . . . . . . . 29

3.6.2 Removal Lemma for families of graphs . . . . . . . . . . . . . . . . . . 29

3.6 .3 Induced Removal Lemma . . . . . . . . . . . . . . . . . . . . . . . 30

4 Property testing and parameter estimation $\quad 31$

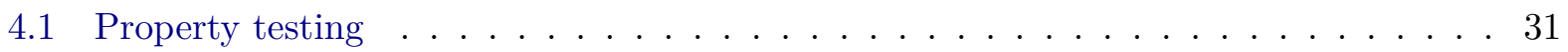

4.1.1 Testability of hereditary properties and Induced Removal Lemmas . . . . . . 33

4.2 Parameter estimation . . . . . . . . . . . . . . . . . 34

4.3 Literature . . . . . . . . . . . . . . . . . . . . . 34

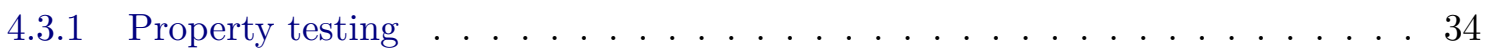

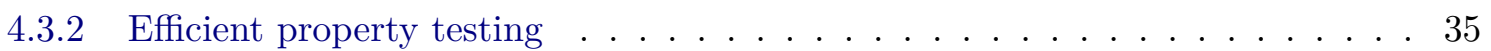

4.3 .3 Parameter estimation . . . . . . . . . . . . . . . 35

4.3.4 Efficient parameter estimation . . . . . . . . . . . . . 36 
$5 \quad$ Partitions of a graph and sampling $\quad 37$

5.1 Related work . . . . . . . . . . . . . . . . . . 38

5.2 Intersection profile with a family of given sets . . . . . . . . . . . . 39

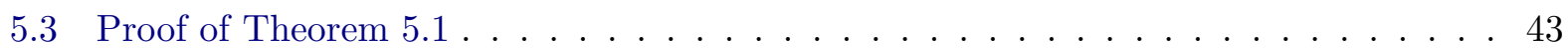

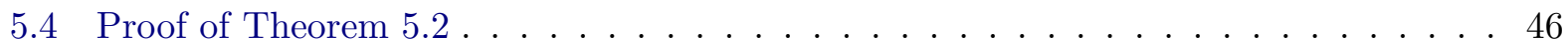

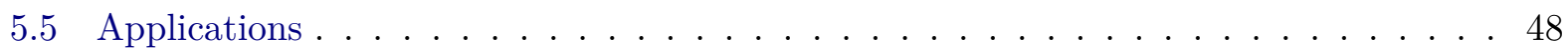

6 Efficient testing of partition problems $\quad 51$

6.1 Fixing regularity with few edge modifications . . . . . . . . . . . . . . 52

6.2 Proof of Theorem $6.3 \ldots \ldots \ldots \ldots \ldots \ldots \ldots$

$\begin{array}{lll}\mathbf{7} & \text { Estimation of parameters by quotient queries } & \mathbf{5 7}\end{array}$

7.1 Parameters given by optimization problems over $G / \wp_{K}^{\leqslant} \ldots \ldots \ldots \ldots$

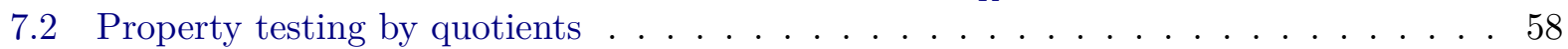

8 Estimating the entropy of monotone graph properties $\quad 61$

8.1 Recoverable graph properties . . . . . . . . . . . . . . . 6 . 63

8.1 .1 Recovering partitions . . . . . . . . . . . . . . 63

8.1 .2 Recoverable properties . . . . . . . . . . . . . . . 64

8.1 .3 Monotone graph properties are recoverable . . . . . . . . . . . . . 64

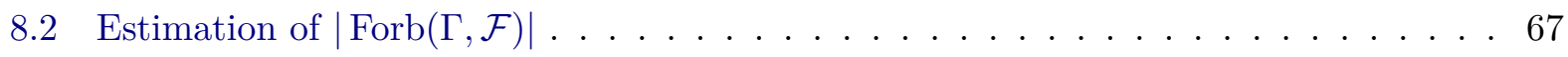

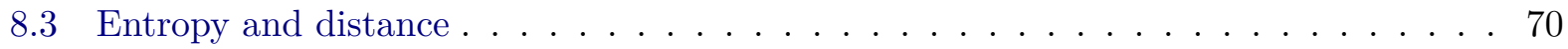

8.4 Discussion . . . . . . . . . . . . . . . . . . . 71

$\begin{array}{lll}9 & \text { Estimation of distance to hereditary graph properties } & \mathbf{7 3}\end{array}$

9.1 Attestable properties . . . . . . . . . . . . . . . . . . . 74

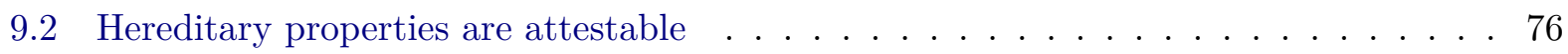

9.3 Shedding some light on shadows f . . . . . . . . . . . . . . . 78

10 On graph properties admitting a proximity oblivious tester $\quad \mathbf{8 1}$

10.1 Proof of Theorem $10.5 \ldots \ldots \ldots \ldots \ldots \ldots$

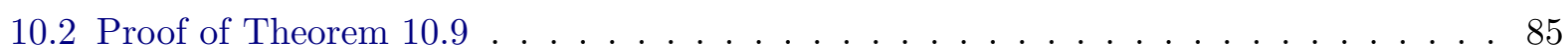

10.3 On the proof of Proposition 3.14 in $[\mathrm{GS} 16] \ldots \ldots \ldots \ldots \ldots$

11 Testing hereditary properties of configurations of points $\quad 91$

11.1 Formal set-up and main results . . . . . . . . . . . . . . . . 92

11.1.1 Property testing for configurations . . . . . . . . . . . . . 93

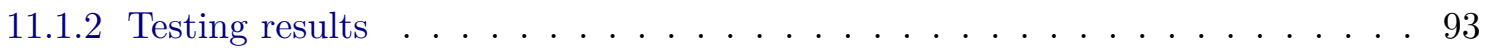

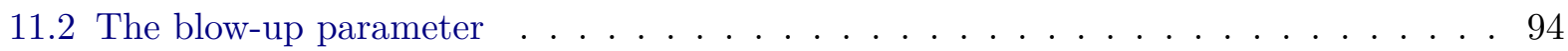

11.3 The Erdős-Hajnal property and upper bounds for $k_{F}^{*} \ldots \ldots \ldots \ldots$

11.4 Proofs of Theorem 11.2 and Theorem $11.3 \ldots \ldots \ldots \ldots$

11.5 Discussion . . . . . . . . . . . . . . . . . . . . . . 99

11.6 Proof sketch for Lemma $11.10 \ldots \ldots$. . . . . . . . . . . . . 99

11.7 The Erdős-Hajnal property and upper bounds for $k_{\mathcal{P}}^{*} \ldots \ldots \ldots$. . . . . . . 99

$\begin{array}{ll}\text { Bibliography } & 103\end{array}$ 
Symbol Index 


\section{Chapter 1}

\section{Introduction}

A graph property $\mathcal{P}$ is said to be testable if, for every $\varepsilon>0$, there exist a positive integer $q_{\mathcal{P}}=q_{\mathcal{P}}(\varepsilon)$, called the query complexity for testing $\mathcal{P}$, and a randomized algorithm $\mathcal{T}_{\mathcal{P}}$, called a tester, which may perform at most $q_{\mathcal{P}}$ queries in the input graph, satisfying the following property. For an $n$-vertex input graph $\Gamma$, the algorithm $\mathcal{T}_{\mathcal{P}}$ distinguishes with probability at least $2 / 3$ between the cases in which $\Gamma$ satisfies $\mathcal{P}$ and in which $\Gamma$ is $\varepsilon$-far from satisfying $\mathcal{P}$, that is, in which no graph obtained from $\Gamma$ by the addition or removal of at most $\varepsilon n^{2} / 2$ edges satisfies $\mathcal{P}$. This may be stated in terms of graph distances: given two graphs $\Gamma$ and $\Gamma^{\prime}$ on the same vertex set $V(\Gamma)=V\left(\Gamma^{\prime}\right)$, we may define the normalized edit distance between $\Gamma$ and $\Gamma^{\prime}$ by $\mathbf{d}_{1}\left(\Gamma, \Gamma^{\prime}\right)=\frac{2}{|V|^{2}}\left|E(\Gamma) \triangle E\left(\Gamma^{\prime}\right)\right|$, where $E(\Gamma) \triangle E\left(\Gamma^{\prime}\right)$ denotes the symmetric difference of their edge sets. If $\mathcal{P}$ is a graph property, we let the distance between a graph $\Gamma$ and $\mathcal{P}$ be

$$
\mathbf{d}_{1}(\Gamma, \mathcal{P})=\min \left\{\mathbf{d}_{1}\left(\Gamma, \Gamma^{\prime}\right): V\left(\Gamma^{\prime}\right)=V(\Gamma) \text { and } \Gamma^{\prime} \in \mathcal{P}\right\}
$$

For instance, if $\Gamma=K_{n}$ and $\mathcal{P}=\operatorname{Forb}\left(K_{3}\right)$, Turán's Theorem ensures that $\left(\begin{array}{l}n \\ 2\end{array}\right)-\left\lfloor n^{2} / 4\right\rfloor$ edges need to be removed to produce a graph that is $K_{3}$-free. In particular, $\mathbf{d}_{1}\left(K_{n}, \operatorname{Forb}\left(K_{3}\right)\right) \rightarrow 1 / 2$. A graph property $\mathcal{P}$ is testable if there is a tester with bounded query complexity that distinguishes with probability at least $2 / 3$ between the cases $\mathbf{d}_{1}(\Gamma, \mathcal{P})=0$ and $\mathbf{d}_{1}(\Gamma, \mathcal{P})>\varepsilon$.

In a similar vein, a function $z: \mathcal{G} \rightarrow \mathbb{R}$ from the set $\mathcal{G}$ of finite graphs into the real numbers is called a graph parameter if it is invariant under relabeling of vertices. A graph parameter $z: \mathcal{G} \rightarrow \mathbb{R}$ is estimable if for every $\varepsilon>0$ and every large enough graph $\Gamma$ with probability at least $2 / 3$, the value of $z(\Gamma)$ can be approximated up to an additive error of $\varepsilon$ by an algorithm that only has access to a subgraph of $\Gamma$ induced by a set of vertices of size $q_{z}=q_{z}(\varepsilon)$, chosen uniformly at random. The query complexity of such an algorithm is $\left(\begin{array}{c}q_{z} \\ 2\end{array}\right)$ and the size $q_{z}$ is called its sample complexity. Estimable parameters have been considered in [FN07] and were defined in the above level of generality in $\left[\mathrm{BCL}^{+} 08\right]$. They are often called testable parameters. Borgs et al. $\left[\mathrm{BCL}^{+} 08\right.$, Theorem 6.1] gave a complete characterization of the estimable graph parameters which, in particular, also implies that the distance from monotone graph properties is estimable. Their work uses the concept of graph limits and does not give explicit bounds on the query complexity required for this estimation.

More precise definitions of the concept of property testing and parameter estimation are given in Chapter 4.

The main contribution of this thesis consists on giving better upper bounds for the sample complexity required to estimate some graph parameters. 
One of the most studied graph parameter in this context is the edit-distance (normalized between 0 and 1) $\mathbf{d}_{\mathcal{P}}: G \mapsto \mathbf{d}_{1}(G, \mathcal{P})$ from an input graph $G=(V, E)$ to a given graph property $\mathcal{P}$, i.e., the minimum fraction of pairs $x y \in V$ whose adjacency must be changed in order to make $G$ satisfy $\mathcal{P}$. A result from Fischer and Newman [FN07] asserts that for every testable property $\mathcal{P}$, the parameter $\mathbf{d}_{\mathcal{P}}$ is estimable. Unfortunately, their result gives an upper bound for the sample complexity required $q(\varepsilon)$ to estimate $\mathbf{d}_{\mathcal{P}}$ is a tower of exponentials whose height is at least the sample complexity required for testing $\mathcal{P}$.

One of the main contributions of this thesis is a result (see Chapter 9, Theorem 9.2) stating that, for any given hereditary graph property $\mathcal{P}$, the sample complexity required to estimate $\mathbf{d}_{\mathcal{P}}$ is at most exponential in the sample complexity required to test $\mathcal{P}$. This represents an important improvement over previous results, specially for properties $\mathcal{P}$ that are known to be efficiently testable. This mentioned result of Chapter 9 is actually a consequence of an intermediate result (Theorem 9.7) giving a bound for estimating the distance to properties $\mathcal{P}$ that are "attestable" — which roughly means that every graph $G \in \mathcal{P}$ has a partition of small size that attests closeness to $\mathcal{P}$ (i.e. such that every graph with a similar partition must be close to some graph $H \in \mathcal{P}$ ). This intermediate result allows us to give even better upper bounds for certain properties.

One of the tools used to prove the results of Chapter 9 consisted in showing that a typical sample of $q$ vertices chosen at random from a graph $G$ provides a good approximation (i.e. such that every density between classes is within an additive error of $\varepsilon$ ) of every partition of a given size $k$. We show in Chapter 5 that this is true, for $q=\operatorname{poly}(\varepsilon, k)$, improving over previous similar results (see [GGR98]), which requires $q$ to be exponential in $k$. Moreover, we extend such results to also relate the partitions of a sample and the partitions of graph not only with respect to the densities, but also with respect to how "regular" they are.

Such results of Chapter 5 also allow us to get better bounds for the sample complexity required to test certain graph properties (see Chapter 6), and certain graph parameters (see Chapter 8).

Finally, by using similar techniques, we were also able to prove negative results about proximityoblivious property testing (which is a more restricted version of property testing) in Chapter 10, disproving a conjecture made by Goldreich and Shinkar in [GS16]. We also used analogous techniques in order to prove results for property testing of configuration of points in the plane (see Chapter 11).

\section{Organization}

This thesis is organized as follows.

We start by given basic definitions and notations that are going to be used throughout the thesis in Chapter 2. General definitions and results about graph regularity are then given in Chapter 3. In Chapter 4, we give the formal definitions related to property testing and parameter estimation and review the main results in the literature.

In Chapter 5 we prove our results relating partitions of a graph $G$ and partitions of a typical sample of $G$.

In Chapter 6 we give the first application of the theorems of Chapter 5. More specifically, we show that the property $\mathcal{P}$ of graphs having a partition with some given densities and regularity constraints is efficiently testable.

To modularize the applications of the results of Chapter 5 in Chapters 8 and 9 , we present two 
intermediate results in Chapter 7.

In Chapter 8 we show that for every graph family $\mathcal{F}$, the parameter $h_{\mathcal{F}}(G)$ that counts the number of spanning subgraphs of $G$ that are free of (not necessarily induced) copies of members of $\mathcal{F}$ is efficiently (with respect to the removal lemma for $\mathcal{F}$ ) estimable.

In Chapter 9, we use techniques similar to Chapter 8 to improve the bounds required to estimate $\mathbf{d}_{\mathcal{P}}$ for any hereditary property $\mathcal{P}$.

We reserve the last two chapters for contributions unrelated with our results from Chapter 5. In Chapter 10, we prove statements about proximity oblivious testing. In Chapter 11, we prove that every hereditary property of configuration of points in the plane is testable.

In the diagram of dependency between chapters below, a highlighted chapter indicates it contains original results developed by the author and co-authors. Chapters 10 and 11 work on notions of property testing which, although formally different from, are closely related to the ones in Chapters 2 to 4 .

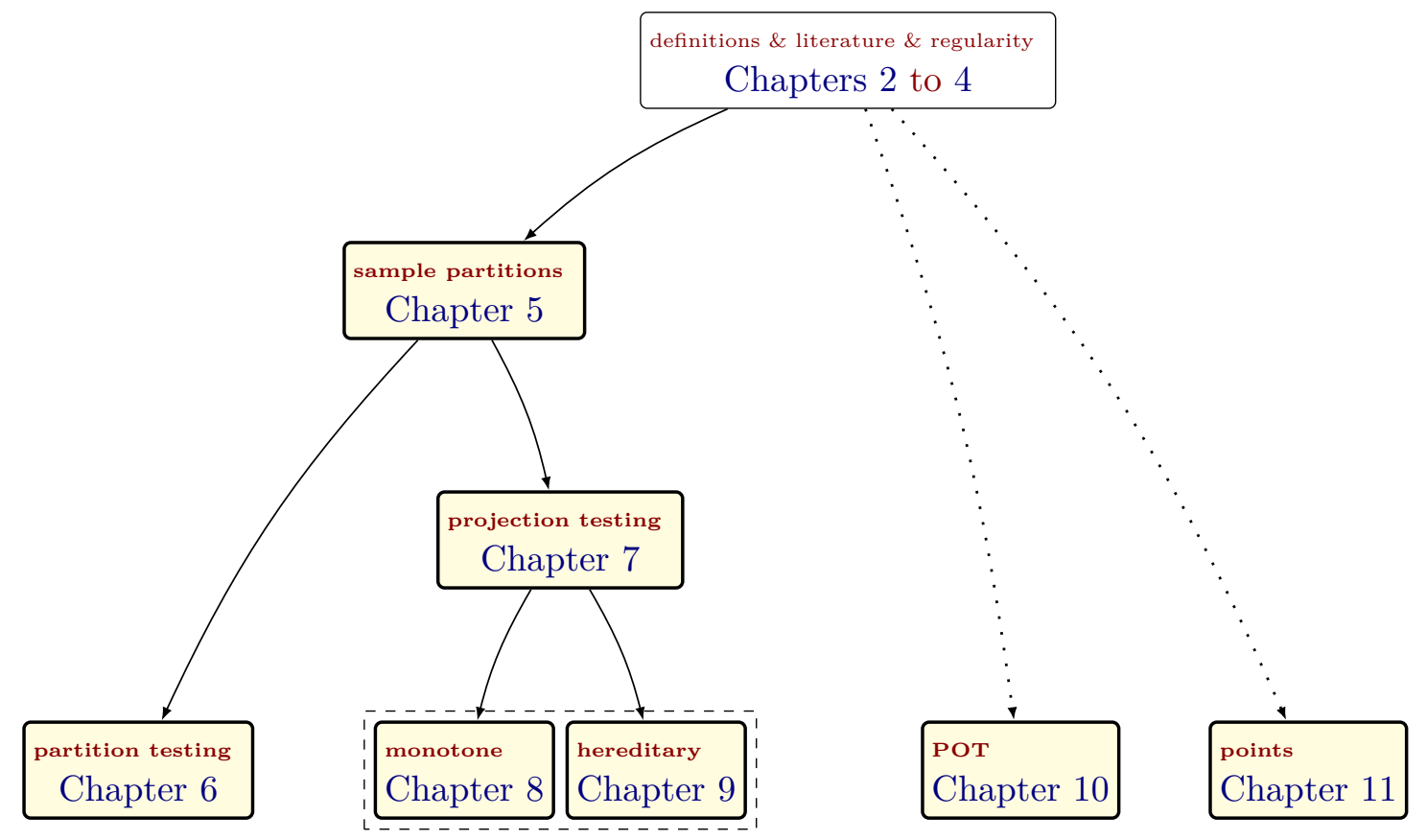

The results in Chapters 5 and 6 will appear in [SS20]. The results in Chapter 8 appeared first in $\left[\mathrm{HKL}^{+} 16\right]$ and then in $\left[\mathrm{HKL}^{+} 20\right]$. The content of Chapter 9 appeared first in $\left[\mathrm{HKL}^{+} 17\right]$ and then in $\left[\mathrm{HKL}^{+}\right]$. Some common material appearing in $\left[\mathrm{HKL}^{+} 16, \mathrm{HKL}^{+} 20, \mathrm{HKL}^{+} 17, \mathrm{HKL}^{+}\right]$is presented in Chapter 7. References [GSS20a, GSS20b] contain the results in Chapter 10. Finally, the content of Chapter 11 appeared in [HKSS18]. 


\section{Chapter 2}

\section{Preliminaries}

Let $V$ be a finite set. The family of all subsets of $V$ of size $q$ will be denoted by $\left(\begin{array}{l}V \\ q\end{array}\right)$. An element $v$ chosen uniformly at random from a set $V$ is denoted by $v \in_{\mathrm{U}} V$. As an example, $Q \in_{\mathrm{U}}\left(\begin{array}{l}V \\ q\end{array}\right)$ is an element of $\left(\begin{array}{l}V \\ q\end{array}\right)$ chosen uniformly at random, i.e., a set of exactly $q$ elements chosen uniformly and without replacement from $V$. Given a subset $S \subseteq V$, we denote $V \backslash S$ simply by $\bar{S}$ whenever there is no chance of confusion regarding the ground set $V$.

If three real numbers $a, b, c$ are such that $|a-b| \leq c$, then we say $a=b \pm c$. The set $\{1,2, \ldots, n\}$ will be denoted by $[n]$.

A pair $(x, y)$ will be denoted simply by $x y$, whenever there is no chance of confusion. Note that, under this notation, $x y$ and $y x$ represent different pairs.

We write $\operatorname{poly}\left(x_{1}, x_{2}, \ldots\right)$ for any quantity $y$ such that $|y| \leq C x_{1}^{a_{1}} x_{2}^{a_{2}} \cdots+b$, where $C, a_{1}, a_{2}, \ldots$ and $b$ are constants. Furthermore, $\operatorname{EXP}\left(x_{1}, x_{2}, \ldots\right)$ stands for any quantity $y$ with $y=2^{O\left(x_{1} \cdot x_{2} \cdots\right)}$.

We set $\operatorname{TOWER}(0)=1$ and, for every $x>0$, we set $\operatorname{TOWER}(x)=2^{\operatorname{TOWER}(\lceil x\rceil-1)}$. Similarly, we set $\operatorname{WOWZER}(0)=1$ and, for every $x>0$, $\operatorname{WOWZER}(x)=\operatorname{TOWER}(\operatorname{WOWZER}(\lceil x\rceil-1))$.

\subsection{Inequalities for random variables}

In what follows, $\mathbb{P}(E)$ denotes the probability of an event $E$ and $\mathbb{E}(X)$ denotes the expected value of a random variable $X$.

Lemma 2.1 (Jensen's inequality). Let $X$ be a random variable and $f: \mathbb{R} \rightarrow \mathbb{R}$ be a convex function. If $\mathbb{E}(f(X))$ and $\mathbb{E}(X)$ are finite, then

$$
\mathbb{E}(f(X)) \geq f(\mathbb{E}(X))
$$

The following is a consequence of Hölder's inequality (for $n$ variables).

Lemma 2.2. Let $\left\{X_{i}\right\}_{i=1}^{n}$ be $n$ random variables with $\mathbb{E}\left(\left|X_{i}\right|^{n}\right)<\infty$ for every $1 \leq i \leq n$. Then

$$
\prod_{i=1}^{n} \mathbb{E}\left(\left|X_{i}\right|^{n}\right) \geq \mathbb{E}\left(\left|\prod_{i=1}^{n} X_{i}\right|\right)^{n} .
$$

Lemma 2.3 (Hoeffding's inequality [Hoe63, Theorem 2]). If $\left\{Z_{i}\right\}_{i=1}^{k}$ are independent random 
variables with $a_{i} \leq Z_{i} \leq b_{i}(i \in[k])$, then for every $t>0$,

$$
\mathbb{P}\left(\left|S_{k}-\mathbb{E}\left(S_{k}\right)\right| \geq t\right) \leq 2 \exp \left\{\frac{-2 t^{2}}{\sum_{i=1}^{k}\left(b_{i}-a_{i}\right)^{2}}\right\}
$$

where $S_{k}=\sum_{i=1}^{k} Z_{i}$.

Hoeffding also discuss in section 6 of [Hoe63] that the same bounds from the previous lemma also holds when each $X_{i}$ comes from sampling from a finite population (in particular, $\left\{X_{i}\right\}_{i=1}^{n}$ are not independent).

Lemma 2.4 (Hoeffding's inequality (sampling without replacement) [Hoe63, Theorem 4]). Let $U=$ $\left\{c_{i}\right\}_{i=1}^{n}$ be a random population of numbers, with $a \leq c_{i} \leq b$ for each $i \in[n]$. Let $\left\{Z_{i}\right\}_{i=1}^{k} \in_{U}\left(\begin{array}{c}U \\ k\end{array}\right)$ be chosen uniformly without replacement from $U$. Then,

$$
\mathbb{P}\left(\left|S_{k}-\mathbb{E}\left(S_{k}\right)\right| \geq t\right) \leq 2 \exp \left\{\frac{-2 t^{2}}{k(b-a)^{2}}\right\}
$$

where $S_{k}=\sum_{i=1}^{k} Z_{i}$.

It is worth mentioning that the random variable $S_{k}$ is actually more concentrated than the one obtained from a sample with replacement, i.e., one can get concentration inequalities that are sharper than Lemma 2.4 (see for instance [BM15]).

The following can be seen as a generalization of Hoeffding's inequality.

Lemma 2.5 (McDiarmid's inequality [McD89, Lemma 1.2]). Let $(\Omega, \mathbb{P})$ be a discrete probability space, let $k$ be a positive integer and $C>0$. Let $Z_{1}, \ldots, Z_{k}$ be independent random variables, where each $Z_{i}$ takes values in some $\Omega_{i}$. Suppose a function $f: \Omega_{1} \times \cdots \times \Omega_{k} \rightarrow \mathbb{R}$ is such that $|f(x)-f(y)| \leq C$ whenever $x=\left(x_{1}, \ldots, x_{k}\right)$ and $y=\left(y_{1}, \ldots, y_{k}\right)$ differ only in one coordinate. Then,

$$
\mathbb{P}(|f(Z)-\mathbb{E}(f(Z))| \geq \lambda) \leq 2 \exp \left\{-\lambda^{2} / 2 k C^{2}\right\} .
$$

As in the case of Hoeffding's inequality, one can get similar bounds when $Z_{1}, \ldots, Z_{k}$ is a sample taken with replacement from a finite population. The following result can be easily obtained from more general results obtained from bounded martingales (see, for instance, [McD89, Theorem 7.4]).

Lemma 2.6 (McDiarmid's inequality (sampling without replacement)). Let $(\Omega, \mathbb{P})$ be a discrete probability space and $k$ be a positive integer and $C>0$. Let $\left\{Z_{1}, \ldots, Z_{k}\right\} \in_{U}\left(\begin{array}{c}V \\ k\end{array}\right)$ be chosen uniformly without replacement from $\Omega$. Suppose a function $f: \Omega_{1} \times \cdots \times \Omega_{k} \rightarrow \mathbb{R}$ is such that $|f(x)-f(y)| \leq C$ whenever $x=\left(x_{1}, \ldots, x_{k}\right)$ and $y=\left(y_{1}, \ldots, y_{k}\right)$ differ only in one coordinate or can be obtained from one another by permuting two coordinates. Then,

$$
\mathbb{P}(|f(Z)-\mathbb{E}(f(Z))| \geq \lambda) \leq 2 \exp \left\{-\lambda^{2} / 2 k C^{2}\right\}
$$

\subsection{Weighted graphs}

Let $Z \subseteq \mathbb{R}$ and let $V$ be a finite set. We assume $V$ has a linear order $<$. We denote by $\mathcal{G}_{Z}(V) \subsetneq Z^{V^{2}}$ $\mathcal{G}_{Z}(V) \quad$ the set of all symmetric matrices $G$ on $V \times V$ with entries in $Z$, i.e., for which $G(x, y)=G(y, x) \in Z$ for every pair $(u, v) \in V^{2}$. 
We will consider an element $G \in \mathcal{G}_{Z}(V)$ as being a $Z$-weighted graph with vertex set $V(G)=V$ and such that every pair $x y$ is associated with a weight $G(x, y) \in Z$.

We will be mainly interested in weighted graphs $G \in \mathcal{G}_{Z}(V)$ for which either

(i) $Z=\{0,1\}$ - in that case $G$ is a "simple graph" (see Section 2.3), and $\mathcal{G}_{\{0,1\}}(V)$ will be denoted simply by $\mathcal{G}(V)$;

(ii) $Z=[0,1]$ - typically when dealing with "reduced graphs" of a simple graph with respect to a partition (see Section 3.1), and $\mathcal{G}_{[0,1]}(V)$ will be denoted simply by $\mathcal{G}^{*}(V)$;

(iii) $Z=[-1,1]$ - typically when considering the difference between two [0,1]-weighted graphs).

Given a weighted graph $R \in \mathcal{G}_{\mathbb{R}}(V)$ and a constant $\eta \in \mathbb{R}$, we write $R+\eta$ to denote the graph $R^{\prime} \in \mathcal{G}_{\mathbb{R}}(V)$ such that $R^{\prime}(u, v)=R(u, v)+\eta$ for every $u, v \in V$.

Given two weighted graphs $R, R^{\prime} \in \mathcal{G}_{\mathbb{R}}(V)$, we write $R \leq R^{\prime}$ if $R(u, v) \leq R^{\prime}(u, v)$ for every $u, v \in V$. If $G, \Gamma \in \mathcal{G}(V)$ are simple graphs for which $G \leq \Gamma$, we say $G$ is a spanning subgraph of $\Gamma$.

For every $G \in \mathcal{G}_{\mathbb{R}}(V)$ and for every two (not necessarily disjoint) subsets $X, Y \subseteq V$, we set

$$
G(X, Y)=\sum_{x y \in(X, Y)} G(x, y)
$$

and we set the density between $X$ and $Y$ as

$$
\mathrm{d}_{G}(X, Y)=\frac{G(X, Y)}{|X||Y|} .
$$

When the underlying graph $G$ is clear from the context, we write $\mathrm{d}_{G}(X, Y)$ simply as $\mathrm{d}(X, Y)$.

Given a graph $G \in \mathcal{G}_{Z}(V)$ and a subset $Q \subseteq V$ of vertices, we define the graph induced by $Q$ as $G[Q]=\left.G\right|_{Q \times Q} \in \mathcal{G}_{Z}(Q)$.

\subsection{Simple graphs}

We define

$$
\mathcal{G}(V)=\left\{G \in \mathcal{G}_{\{0,1\}}(V): G(x, x)=0 \text { for every } x \in X\right\}
$$

as the set of graphs (or simple graphs when we feel the need of emphasizing) with vertex set $V$. We also define $\mathcal{G}=\bigcup_{V} \mathcal{G}(V)$ for every $V$ finite. The set of edges of $G \in \mathcal{G}(V)$ will be defined as

$$
E(G)=\{x y: G(x, y)=1 \text { and } x<y\}
$$

We also denote by $G=(V, E)$ a graph $G$ for which $V(G)=V$ and $E(G)=E$.

Given a graph $G=(V, E)$ and two (not necessarily disjoint) sets $A, B \subseteq V$, we set e $(A, B)=$ $G(A, B)$ (note that if $A \cap B=\emptyset$, then e $(A, B)$ count the number of edges between $A$ and $B)$. We also set $\mathrm{e}(G)=\mathrm{e}(V, V) / 2=|E|$ as the number of edges of $G$.

Two graphs $G=(V, E)$ and $G^{\prime}=\left(V^{\prime}, E^{\prime}\right)$ are isomorphic if there is a bijection $\varphi: V \hookrightarrow V^{\prime}$ preserving adjacency, i.e. for which $u v \in E$ if and only if $\varphi(u) \varphi(v) \in E^{\prime}$ for every $u, v \in V$.

Let $G=(V, E)$ and $F=\left([f], E_{F}\right)$ be graphs and let $U \subseteq V$ be a set of size $|U|=f$. We say $U$ spans an induced copy of $F$ in $G$ if $G[U]$ is isomorphic to $F$. We say $U$ spans a copy of $F$ in $G$ if $G[U]$ is isomorphic to some graph $F^{\prime} \geq F$. 
The automorphism group $\operatorname{Aut}(G)$ of a graph $G=(V, E)$ is the group of permutations of $V$ preserving adjacency, i.e

$$
\operatorname{Aut}(G)=\{\sigma: V \hookrightarrow V: u v \in E \Leftrightarrow \sigma(u) \sigma(v) \in E\}
$$

$\operatorname{aut}(G) \quad$ We also set $\operatorname{aut}(G)$ as the number $|\operatorname{Aut}(G)|$ of automorphisms of $G$.

\subsection{Bipartite graphs}

$\mathcal{H}_{Z}(X, Y) \quad$ Let $Z \subseteq \mathbb{R}$ and let $X$ and $Y$ be finite sets. We denote by $\mathcal{H}_{Z}(X, Y)=Z^{X \times Y}$ the set of all matrices $H$ over $X \times Y$ with entries in $Z$.

The elements of $\mathcal{H}_{Z}(X, Y)$ are called $Z$-weighted bipartite graphs. Elements of $\mathcal{H}_{\{0,1\}}(X, Y)$ are $\mathcal{H}(X, Y) \quad$ called bipartite graphs and we set $\mathcal{H}(X, Y):=\mathcal{H}_{\{0,1\}}(X, Y)$.

$E(H), \mathrm{e}(H) \quad$ Let $H \in \mathcal{H}(X, Y)$ be a bipartite graph. We set $E(H)=\{x y: H(x, y)=1\}$ and we denote $H$ $H=(X, Y, E) \quad$ by $H=(X, Y, E(H))$. As in the case of graphs, we also set $\mathrm{e}(H)=|E(H)|$.

Bipartite graphs, as defined in this section, will be used mainly to focus on the edges between two given sets of a graph. More precisely, given a graph $G \in \mathcal{G}_{Z}(V)$ and two (not necessarily disjoint)

$G[X, Y] \quad$ sets $X, Y \subseteq V$, then we define the induced bipartite graph $G[X, Y]:=\left.G\right|_{X, Y} \in \mathcal{H}_{Z}(X, Y)$. For instance, if $G=(V, E)$ and $X, Y \subseteq V$ are two (not necessarily disjoint) sets of vertices, then we have $G[X, Y]:=\left(X, Y,\left.E\right|_{X \times Y}\right)$, where $\left.E\right|_{X \times Y}=\{x y \in E: x \in X, y \in Y\}$. If the graph $G$ is clear from context, we simply write the pair $(X, Y)$ to refer to the bipartite graph $G[X, Y]$.

Remark 2.7. We stress that the term "bipartite graph" is not being used here as a synonym for "2-colorable graph".

$C_{4} \quad$ Remark 2.8. The 4-cycle $C_{4}=\mathbb{Q}_{0}^{\circ}$ will occur in this thesis in several places. It will be clear 送 from the context whether we are considering $C_{4}$ as a member of, say, $\mathcal{G}([4])$ or as a member of, say, $\mathcal{H}([2],[2])$.

Let $H \in \mathcal{H}_{Z}(X, Y)$. As in the case of graphs we write

$H(X, Y)$

$$
H(A, B)=\sum_{a \in A, b \in B} H(a, b)
$$

for every $A \subseteq X$ and $B \subseteq Y$. For every $X^{\prime} \subseteq X$ and $Y^{\prime} \subseteq Y$ we define $H\left[X^{\prime}, Y^{\prime}\right]=\left.H\right|_{X^{\prime} \times Y^{\prime}} \in$ $\mathcal{H}_{Z}\left(X^{\prime}, Y^{\prime}\right)$.

$\mathrm{d}(H) \quad$ We define the density $\mathrm{d}(H)$ of a bipartite graph as $\mathrm{d}(H):=|E(H)| /|X||Y|$.

Remark 2.9. We observe that, formally, a graph $G \in \mathcal{G}(V)$ can also be considered as a bipartite graph $H \in \mathcal{H}(V, V)$, with $H(u, v)=G(u, v)$, for every $u, v \in V$. Naturally, in this bipartite graph $H$, we have $H(u, v)=H(v, u)$ for every $u, v \in V$ and $H(u, u)=0$ for every $u \in V$.

\subsection{Graph properties}

A graph property is a set of graphs $\mathcal{P} \subseteq \mathcal{G}$ which is invariant under isomorphisms. 
A graph property $\mathcal{P}$ is monotone if it is closed under the removal of vertices and edges. The property

$$
\operatorname{Forb}(F)=\{G \in \mathcal{G}: G \text { has no copy of } F\}
$$

of graphs having no copies of some graph $F$ is a typical example of a monotone graph property. For every (possibly infinite) family $\mathcal{F}$ of graphs, let

$$
\operatorname{Forb}(\mathcal{F})=\bigcap_{F \in \mathcal{F}} \operatorname{Forb}(F)
$$

be the property of graphs with no copies of members of $\mathcal{F}$. Then every monotone property $\mathcal{P}$ can be written as $\operatorname{Forb}(\mathcal{F})$ for some family $\mathcal{F}$ - in particular one can take $\mathcal{F}$ to be the family of all edge-minimal graphs not in $\mathcal{P}$.

A graph property $\mathcal{P}$ is hereditary if it is inherited by induced subgraphs - i.e, if $G \in \mathcal{P}$ implies $G[S] \in \mathcal{P}$ for every $S \subseteq V(G)$. The property

$$
\operatorname{Forb}^{\bullet}(F)=\{G \in \mathcal{G}: G \text { has no induced copy of } F\}
$$

of graphs having no induced copies of some graph $F$ is a typical example of a hereditary graph property. For every (possibly infinite) family $\mathcal{F}$ of graphs, let

$$
\operatorname{Forb}^{\bullet}(\mathcal{F})=\bigcap_{F \in \mathcal{F}} \operatorname{Forb}^{\bullet}(F)
$$

Every hereditary property $\mathcal{P}$ can be written as $\operatorname{Forb}^{\bullet}(\mathcal{F})$ for some family $\mathcal{F}$ - in particular one can take $\mathcal{F}$ to be the family of all vertex-minimal graphs not in $\mathcal{P}$.

\subsection{Graph norm and distance}

For any given $G, H \in \mathcal{G}_{\mathbb{R}}(V)$, with $|V|=n$, define the $\langle G, H\rangle$ as

$$
\langle G, H\rangle=\frac{1}{n^{2}} \sum_{x y \in V^{2}} G(x, y) H(x, y)
$$

and the $\ell_{1}$ and $\ell_{2}$ norms of a graph $G_{\mathbb{R}}(V)$ as

$$
\|G\|_{1}=\frac{1}{n^{2}} \sum_{x y \in V^{2}}|G(x, y)| \text { and }\|G\|_{2}=\langle G, G\rangle^{\frac{1}{2}} .
$$

respectively.

We define the distance between two graphs $G$ and $H$ with $V(G)=V(H)$ as

$$
\mathbf{d}_{1}(G, H)=\|G-H\|_{1}=\frac{1}{n^{2}} \sum_{x y \in V^{2}}|G(x, y)-H(x, y)|,
$$

where $n=|V(G)|=|V(H)|$. Note that for the particular case in which $G=\left(V, E_{G}\right)$ and $H=\left(V, E_{H}\right)$ 
are simple graphs, then

$$
\mathbf{d}_{1}(G, H)=\frac{2\left|E_{G} \triangle E_{H}\right|}{|V|^{2}}=\left(\begin{array}{c}
|V| \\
2
\end{array}\right)^{-1}\left|E_{G} \triangle E_{H}\right|+O\left(\frac{1}{n}\right),
$$

where $\triangle$ denotes the symmetric difference operator.

Note that $\mathbf{d}_{1}(G, H)$ may be strictly positive even if $G$ and $H$ are isomorphic. One can define the distance between the isomorphism classes of $G$ and $H$ as $\min _{H^{\prime}} \mathbf{d}_{1}\left(G, H^{\prime}\right)$, where the minimum is taken over all isomorphic copies $H^{\prime}$ of $H$ on $V=V(G)=V(H)$. In this thesis, we will not need this concept.

The cut norm of a weighted graph $G \in \mathcal{G}_{\mathbb{R}}(V)$, with $|V|=n$, is defined as

$\|G\|_{\square}$

$$
\|G\|_{\square}=\frac{1}{n^{2}} \max _{S, T \subseteq V}|G(S, T)| .
$$

The following proposition asserts the cut norm is essentially equivalent to a norm restricted to cuts of the form $G(S, S)$.

Proposition 2.10. For every graph $G \in \mathcal{G}_{\mathbb{R}}(V)$,

$$
\|G\|_{\square} \leq\|G\|_{\square} \leq 2\|G\|_{\square}
$$

where

$\|G\|_{\Xi}$

$$
\|G\|_{\square}=\max _{S \subseteq V} \frac{|G(S, S)|}{n^{2}} .
$$

Proof. Let $S, T \subseteq V$ be such that $\|G\|_{\square}=G(S, T) / n^{2}$. Then, by the inclusion-exclusion principle,

$$
\begin{aligned}
2\|G\|_{\square} n^{2}=G(S, T) & +G(T, S) \\
& =G(S \cup T, S \cup T)-G(S \backslash T, S \backslash T)-G(T \backslash S, T \backslash S)+G(S \cap T, S \cap T) .
\end{aligned}
$$

Hence there must be $A \in\{S \cup T, S \backslash T, T \backslash S, S \cap T\}$ for which $|G(A, A)| \geq \frac{2}{4}\|G\|_{\square} n^{2}$, as required.

Definition 2.11. For every graph $G \in \mathcal{G}_{[-1,1]}(V)$, define

$\|G\|_{\square_{f, g}^{+}}$

$$
\|G\|_{\square_{f, g}^{+}}=\frac{1}{|V|^{2}} \max _{f, g}\left|\sum_{\substack{x \in V \\ y \in V}} f(x) \cdot G(x, y) \cdot g(y)\right|
$$

where the maximum is taken over all function $f, g \in V \rightarrow[0,1]$. For every graph $G \in \mathcal{G}_{[-1,1]}(V)$, define

$$
\|G\|_{\square_{f, g}}=\frac{1}{|V|^{2}} \max _{f, g}\left|\sum_{\substack{x \in V \\ y \in V}} f(x) \cdot G(x, y) \cdot g(y)\right|
$$

where the maximum is taken over all function $f, g \in V \rightarrow[-1,1]$.

Remark 2.12. If $H \in \mathcal{H}_{[-1,1]}(X, Y)$ is a bipartite graph, then one can also define $\|H\|_{\square},\|H\|_{\square_{f, g}^{+}}$ and $\|H\|_{\square_{f, g}}$ analogously to Definition 2.11 .

Lemma 2.13 (see [Lov12, Lemma 8.11]). For every graph $G \in \mathcal{G}_{[-1,1]}(V)$,

$$
\|G\|_{\square}=\|G\|_{\square_{f, g}^{+}} \geq \frac{1}{4}\|G\|_{\square_{f, g}} .
$$


Likewise, for every bipartite graph $H \in \mathcal{H}_{[-1,1]}(X, Y)$,

$$
\|H\|_{\square}=\|H\|_{\square_{f, g}^{+}} \geq \frac{1}{4}\|H\|_{\square_{f, g}} .
$$

\subsection{Homomorphisms of graphs}

Let $F=(V(F), E(F))$ and $G \in(V(G), E(G))$ be graphs. A function $\varphi: V(F) \rightarrow V(G)$ is called a graph homomorphism from $F$ into $G$ if $\varphi$ preserves adjacency, i.e., if $\varphi(u) \varphi(v) \in E(G)$ for every $u v \in E(F)$ In that case we simply write $F \rightarrow G$. Note that since $F$ and $G$ are simple graphs, the definition above implies, in particular, that a graph homomorphism from $F$ into $G$ cannot map two adjacent vertices of $F$ to the same vertex of $G$ (because $G$ has no loops).

If there is a homomorphism $\psi$ from $F$ into $F^{\prime}$ and $\psi$ is surjective, then $F^{\prime}$ is called a homomorphic copy of $F$. In particular, if $\varphi: V(F) \rightarrow V(G)$ is a graph homomorphism, then the graph $G[\Im \varphi]$ spanned by the vertices in the image of $\varphi$ is a homomorphic copy of $F$. Moreover, if $\varphi$ is injective then then $(\varphi(u))_{u \in V(F)} \in V(G)^{V(F)}$ is called a labeled copy of $F$ in $G$.

A function $\varphi: V(F) \rightarrow V(G)$ is called an induced graph homomorphism from $F$ into $G$ if $\varphi(u) \varphi(v) \in E(G)$ if, and only if, $u v \in E(F)$. The definitions of induced homomorphic copy and induced labeled copy are defined analogously.

Given two graphs $F$ and $G$, one can consider the probability that a random mapping $\varphi: V(F) \rightarrow$ $V(G)$ is a (induced) graph homomorphism. The following section defines these two quantities formally in the more general case in which $G$ is a weighted graph.

\subsubsection{Homomorphism density for graphs}

In this section, let $F=([f], E(F))$ be a graph and let $G \in \mathcal{G}_{\mathbb{R}}(V)$ be a weighted graph.

For any given $\varphi:[f] \rightarrow V$ we define the homomorphism weight $\mathrm{hw}_{\varphi}(F ; G)$ as

$$
\operatorname{hw}_{\varphi}(F ; G)=\prod_{i j \in E(F)} G(\varphi(i), \psi(j))
$$

and the induced homomorphism weight $\operatorname{hw}_{\varphi}^{\mathbf{e}}(F ; G)$ as

$$
\operatorname{hw}_{\varphi}^{\boldsymbol{e}}(F ; G)=\prod_{i j \in E(F)} G(\varphi(i), \psi(j)) \prod_{i j \in E(\bar{F})}(1-G(\varphi(i), \psi(j))) .
$$

Note that for the particular case in which $G \in \mathcal{G}(V)$ is a simple graph, we have $\operatorname{hw}_{\varphi}(F ; G)=1$ if $\varphi$ is a homomorphism from $F$ into $G$ and $\operatorname{hw}_{\varphi}(F ; G)=0$ otherwise.

Next, we define the homomorphism density of $F$ in $G$ as

$$
\mathrm{t}(F ; G)=\frac{1}{|V|^{f}} \sum_{\varphi:[f] \rightarrow V} \operatorname{hw}_{\varphi}(F ; G),
$$

i.e., as the average of $\mathrm{hw}_{\varphi}(F ; G)$ over all mappings $\varphi:[f] \rightarrow V(G)$. We also define the injective homomorphism density of $F$ in $G$ as

$$
\mathrm{t}_{\hookrightarrow}(F ; G)=\frac{1}{(|V|)_{f}} \sum_{\varphi:[f] \hookrightarrow V} \operatorname{hw}_{\varphi}(F ; G),
$$


i.e., as the average of $\operatorname{hw}_{\varphi}(F ; G)$ over all injective mappings $\varphi$ : $[f] \hookrightarrow V(G)$.

In particular, if $G \in \mathcal{G}(V)$, then $\mathrm{t}(F ; G)$ is the probability that a random mapping $\varphi:[f] \rightarrow V$ is a homomorphism of $F$ into $G$. Likewise, $\mathrm{t}_{\iota}(F ; G)$ is the probability that a sequence $\left(v_{i}\right)_{i=1}^{f} \in V^{[f]}$ of vertices of $V$ chosen at random without replacement is a labeled copy of $F$ in $G$. Sometimes, it $\operatorname{Hom}(F, G) \quad$ will be convenient to consider the total number $\operatorname{Hom}(F, G):=\mathrm{t}(F ; G) n^{f}$ of homomorphisms from $F$ to $G$.

We also define the quantities

$\mathrm{t}^{\bullet}(F ; G)$

$$
\mathrm{t}^{\boldsymbol{e}}(F ; G)=\frac{1}{|V|^{f}} \sum_{\varphi:[f] \rightarrow V} \operatorname{hw}_{\varphi}^{\boldsymbol{e}}(F ; G)
$$

and

$\mathrm{t}_{\leftrightarrow}^{\mathbf{e}}(F ; G)$

$$
\mathrm{t}_{\hookrightarrow}^{\boldsymbol{\Theta}}(F ; G)=\frac{1}{(|V|)_{f}} \sum_{\varphi:[f] \hookrightarrow V} \operatorname{hw}_{\varphi}^{\boldsymbol{\Theta}}(F ; G)
$$

analogously for the induced case. Note that if $G \in \mathcal{G}(V)$, then $\mathrm{t}_{\hookrightarrow}^{\mathbf{0}}(F ; G)$ is the probability that a sequence $\left(v_{1}, \ldots, v_{f}\right)$ of vertices of $V$ chosen at random without replacement spans an induced labeled copy of $F$ in $G$.

Finally, we define the induced density of $F$ in $G$ as

$\mathrm{p}^{\bullet}(F ; G)$

$$
\mathrm{p}^{\bullet}(F ; G)=\frac{f !}{\operatorname{aut}(F)} \mathrm{t}_{\leftarrow}^{\bullet}(F ; G)
$$

and the density of $F$ in $G$ as

$\mathrm{p}(F ; G)$

$$
\mathrm{p}(F ; G)=\sum_{F^{\prime}: F^{\prime} \geq F} \mathrm{p}^{\bullet}\left(F^{\prime} ; G\right) .
$$

Note that if $G \in \mathcal{G}(V)$ is a graph, then $\mathrm{p}^{\bullet}(F ; G)$ is the probability that a set $U \in_{\mathrm{U}}\left(\begin{array}{l}V \\ f\end{array}\right)$ spans an induced copy of $F$, since the number of induced copies of $F$ in $G$ is given by $f !\left(\begin{array}{c}|V| \\ f\end{array}\right) \mathrm{t}_{\hookrightarrow}^{\mathbb{O}}(F ; G)$. Likewise, $\mathrm{p}(F ; G)$ is the probability that such a $U$ spans a copy of $F$.

\subsubsection{Homomorphism density for bipartite graphs}

It will be useful to extend the definitions of Section 2.7.1 for bipartite graphs. In what follows, let $F=([s],[t], E(F))$ be a bipartite graph and $H \in \mathcal{H}_{[-1,-1]}(X, Y)$ be a weighted bipartite graph.

For any given $\varphi:[s] \rightarrow X$ and $\psi:[t] \rightarrow Y$ we define the homomorphism weight $\mathrm{hw}_{\varphi, \psi}(F ; H)$ as

$\operatorname{hw}_{\varphi, \psi}(F ; H)$

$$
\operatorname{hw}_{\varphi, \psi}(F ; H)=\prod_{i j \in E(F)} H(\varphi(i), \psi(j))
$$

and the induced homomorphism weight $\mathrm{hw}_{\varphi, \psi}^{\boldsymbol{e}}(F ; H)$ as

$$
\operatorname{hw}_{\varphi, \psi}^{\bullet}(F ; H)=\prod_{i j \in E(F)} H(\varphi(i), \psi(j)) \prod_{i j \in E(\bar{F})}(1-H(\varphi(i), \psi(j))) .
$$

Then the homomorphism density $\ddot{\mathrm{t}}(F ; H)$ and the injective homomorphism density $\ddot{\mathrm{t}}_{\iota}(F ; H)$ are 
defined analogously to their respective counterparts. More precisely, we set

$$
\begin{aligned}
& \ddot{\mathrm{t}}(F ; H)=\frac{1}{|X|^{s}|Y|^{t}} \sum_{\substack{\varphi:[s] \rightarrow X \\
\psi:[t] \rightarrow Y}} \operatorname{hw}_{\varphi, \psi}(F ; H), \\
& \ddot{\mathrm{t}}_{\hookrightarrow}(F ; H)=\frac{1}{(|X|)_{s}(|Y|)_{t}} \sum_{\substack{\varphi:[s] \hookrightarrow X \\
\psi:[t] \hookrightarrow Y}} \operatorname{hw}_{\varphi, \psi}(F ; H), \\
& \ddot{\mathrm{t}}^{\bullet}(F ; H)=\frac{1}{|X|^{s}|Y|^{t}} \sum_{\substack{\varphi:[s] \rightarrow X \\
\psi:[t] \rightarrow Y}} \operatorname{hw}_{\varphi, \psi}^{\boldsymbol{e}}(F ; H), \\
& \ddot{\mathrm{t}}_{\hookrightarrow}^{\boldsymbol{\Theta}}(F ; H)=\frac{1}{(|X|)_{s}(|Y|)_{t}} \sum_{\substack{\varphi:[s] \hookrightarrow X \\
\psi:[t] \hookrightarrow Y}} \operatorname{hw}_{\varphi, \psi}^{\boldsymbol{e}}(F ; H) .
\end{aligned}
$$

For the bipartite case, we will mainly make use of $\ddot{\mathrm{t}}(F ; H)$ (in particular for $F=C_{4}=\mathbb{Z}_{0}^{0}$, see Definition 3.7), we will use the shorter notation $\ddot{\mathrm{t}}_{F}(H):=\ddot{\mathrm{t}}(F ; H)$.

Also, for $A \subseteq X$ and $B \subseteq Y$ we will write $\ddot{\mathrm{t}}_{F}(H[A, B])$ simply as $\ddot{\mathrm{t}}_{F}(A, B)$, when there is no chance of confusion about the underlying graph $H$.

\subsection{Positive correlation between homomorphism densities}

The following discussion is related to the well known conjecture of Sidorenko (see [Sid91, Sid93]). Let $F=([s],[t], E)$ be a bipartite subgraph of $K_{s, t}$. We write $F=\biguplus_{k=1}^{m} F_{k}$ if there are graphs $F_{k}=$ $\left([s],[t], E_{k}\right)$ with disjoint edge sets whose union $\bigcup_{k=1}^{m} E_{k}$ is $E$. One could ask for which such decompositions $F=\bigcup_{k=1}^{m} F_{k}$ the following holds:

$$
\ddot{\mathrm{t}}_{F}(H) \geq \prod_{k=1}^{m} \ddot{\mathrm{t}}_{F_{k}}(H), \quad \text { for every bipartite graph } H \in \mathcal{H}(X, Y) \text {. }
$$

Although assertion (2.1) does not hold in general (see e.g. [Lon66] for a counterexample for $F=$ $P_{4}=P_{3} \cup P_{2}$ ), there are many particular cases for which it does. In particular, Sidorenko's conjecture ([Sid93, Conjecture 1]) claims assertion (2.1) holds for every decomposition $F=\biguplus_{k=1}^{m} F_{k}$ with $\mathrm{e}\left(F_{k}\right)=1$ for all $k \in[m]$, which translates simply to $\ddot{\mathrm{t}}_{F}(H) \geq \mathrm{d}(H)^{\mathrm{e}(F)}$ for every bipartite graph $H$. The following proposition asserts assertion (2.1) does hold whenever $F=K_{s, t}$.

Lemma 2.14. For every decomposition $K_{s, t}=\bigcup_{k=1}^{m} F_{k}$ and every bipartite graph $H$,

$$
\ddot{\mathrm{t}}_{K_{s, t}}(H) \geq \prod_{k=1}^{m} \ddot{\mathrm{t}}_{F_{k}}(H) \text {. }
$$

In particular, for every subgraph $F$ of $K_{2,2}=\mathfrak{Q}_{0}^{\circ}$, we have

$$
\ddot{\mathrm{t}}_{K_{2,2}}(H) \geq \ddot{\mathrm{t}}_{F}(H) \mathrm{d}(H)^{4-\mathrm{e}(F)}
$$

Proof. First, we remark that ineq. (2.3) indeed follows from ineq. (2.2) by considering the decomposition $K_{2,2}=F \bullet \biguplus_{e \notin E(F)}([2],[2],\{e\})$. We will derive ineq. (2.2) by showing that the following 
holds for every subgraph $F$ of $K_{s, t}$ :

$$
\ddot{\mathrm{t}}_{K_{s, t}}(H)^{\mathrm{e}(F)} \geq \ddot{\mathrm{t}}_{F}(H)^{s t}, \quad \text { for every bipartite graph } H \text {. }
$$

Indeed, by applying the above assertion for each $F_{k}$ and multiplying the respective inequalities, one gets ineq. (2.2). Assertion (2.4) is actually a consequence of a much more general results in [Hat10]. Because of the more general and complex setup in [Hat10], we prove assertion (2.4) below for the reader's convenience.

Suppose $F=([s],[t], E(F))$ and for each $j \in[t]$ define $N(j)=\{i \in[s]: i j \in E(F)\}$ as the neighborhood of a vertex $j \in[t]$. Let $x_{1}, \ldots x_{s} \in X$ and $y_{1}, \ldots, y_{t} \in Y$ be chosen independently and uniformly at random. In what follows, we denote $\left(x_{1}, \ldots, x_{s}\right)$ and $\left(y_{1}, \ldots, y_{t}\right)$ by $\bar{x}$ and $\bar{y}$, respectively. For each $j \in[t]$, define

$$
\mu_{N(j)}=\frac{1}{|Y|} \mid\left\{y \in Y: x_{i} y \in E(H) \text { for every } i \in N(j)\right\}
$$

as the random variable (on $\bar{x}$ ) which evaluates to the fraction of vertices $y \in Y$ that, together with $\bar{x}$, span a homomorphic copy of $K_{|N(j)|, 1}$. We have

$$
\ddot{\mathrm{t}}_{F}(H)=\mathbb{P}_{\bar{x}, \bar{y}}\left(\bigwedge_{j=1}^{t}\left[x_{i} y_{j} \in E(H): \forall i \in N(j)\right]\right)=\mathbb{E}_{\bar{x}} \mathbb{E}_{\bar{y}}\left(\prod_{j=1}^{t} \mathbb{1}_{\left[x_{i} y_{j} \in E(H): \forall i \in N(j)\right]}\right) .
$$

Since, for a given $\bar{x}$, the events $\left[x_{i} y_{j} \in E(H): \forall i \in N(j)\right]$ are all independent, we get

$$
\left.\ddot{\mathrm{t}}_{F}(H)=\mathbb{E}_{\bar{x}}\left(\prod_{j=1}^{t} \mathbb{E}_{\bar{y}} \mathbb{1}_{\left[x_{i} y_{j} \in E(H): \forall i \in N(j)\right]}\right)\right)=\mathbb{E}_{\bar{x}}\left(\prod_{j=1}^{t} \mu_{N(j)}\right) .
$$

Now we take the previous equality to the $t$-th power and apply Hölder's inequality to get

$$
\ddot{\mathrm{t}}_{F}(H)^{t}=\left(\mathbb{E}_{\bar{x}}\left(\prod_{j=1}^{t} \mu_{N(j)}\right)\right)^{t} \leq \prod_{j=1}^{t} \mathbb{E}_{\bar{x}} \mu_{N(j)}^{t}=\prod_{j=1}^{t} \ddot{\mathrm{t}}_{F_{j}}(H)
$$

where the equality is simply $(2.5)$ applied to $F_{j}:=([s],[t], N(j) \times[t])$, i.e., the complete graph between $N(j)$ and $[t]$. Next, define the random variable (analogous to $\mu$ )

$$
\nu_{[t]}=\frac{1}{|X|} \mid\left\{x \in X: x y_{j} \in E(H) \text { for every } j \in[t]\right\}
$$

One can verify that $\ddot{t}_{F_{j}}(H)=\mathbb{E}_{\bar{y}} \nu_{[t]}^{|N(j)|}$ by an argument analogous to (2.5) applied to $F_{j}$. After substituting in ineq. (2.6), we get

$$
\ddot{\mathrm{t}}_{F}(H)^{t} \leq \prod_{j=1}^{t} \ddot{\mathrm{t}}_{F_{j}}(H)=\prod_{j=1}^{t} \mathbb{E}_{\bar{y}} \nu_{[t]}^{|N(j)|} \leq \prod_{j=1}^{t}\left(\mathbb{E}_{\bar{y}} \nu_{[t]}^{s}\right)^{N(j) / s}=\left(\mathbb{E}_{\bar{y}} \nu_{[t]}^{s}\right)^{\mathrm{e}(F) / s}
$$

where the inequality follows from the concavity of the function $x \mapsto x^{N(j) / s}$ (Lemma 2.1). It is not difficult to see that $\mathbb{E}_{\bar{y}} \nu_{[t]}^{s}=\ddot{\mathrm{t}}_{K_{s, t}}(H)$, again by applying to $K_{s, t}$ an argument analogous to (2.5). Therefore, we get $\ddot{t}_{F}(H)^{s t} \leq \ddot{\mathrm{t}}_{K_{s, t}}(H)$, as desired. 
Moreover, recall that (see Remark 2.9) every graph $G \in \mathcal{G}(V)$ is also a bipartite graph $G \in$ $\mathcal{H}(V, V)$. When considering $G$ as a member of $\mathcal{H}(V, V)$, we write $\mathcal{H}(G)$ for $G$. In the other direction, for any bipartite graph $F \in \mathcal{H}([s],[t])$, one can consider the (2-colorable) graph $\mathcal{G}(F)=([s] \sqcup[t],\{i j$ : $(i, j) \in E(F))$. The following is a corollary of Lemma 2.14 .

Lemma 2.15. For every decomposition $K_{s, t}=\biguplus_{k=1}^{m} F_{k} \in \mathcal{H}([s],[t])$ and every graph $G \in \mathcal{G}(V)$,

$$
\mathrm{t}\left(\mathcal{G}\left(K_{s, t}\right) ; G\right) \geq \prod_{k=1}^{m} \mathrm{t}\left(\mathcal{G}\left(F_{k}\right) ; G\right)
$$

In particular, for every subgraph $F$ of $C_{4}=\mathcal{G}\left(K_{2,2}\right)$, we have

$$
\mathrm{t}\left(C_{4} ; G\right) \geq \mathrm{t}(F ; G) \mathrm{d}(H)^{4-\mathrm{e}(F)} .
$$

Proof. Note that for every $F \in \mathcal{H}([s],[t])$, the homomorphic density $\ddot{\mathrm{t}}_{F}(\mathcal{H}(G))$ corresponds to the probability of choosing sequences of vertices $\left(x_{i}\right)_{i=1}^{s} \in_{\mathrm{U}} V^{s}$ and $\left(y_{j}\right)_{j=1}^{t} \in_{\mathrm{U}} V^{t}$ independently at random and having $x_{i} y_{j} \in E(G)$ for every $i j \in E(F)$. That probability, in turn, is exactly $\mathrm{t}(\mathcal{G}(F) ; G)$. Hence, we have $\ddot{\mathrm{t}}_{F}(\mathcal{H}(G))=\mathrm{t}(\mathcal{G}(F) ; G)$ for every $F \in \mathcal{H}([s],[t])$.

Then, it follows from Lemma 2.14 that

$$
\mathrm{t}\left(\mathcal{G}\left(K_{s, t}\right) ; G\right)=\ddot{\mathrm{t}}_{K_{s, t}}(\mathcal{H}(G)) \geq \prod_{k=1}^{m} \ddot{\mathrm{t}}_{F_{k}}(\mathcal{H}(G))=\prod_{k=1}^{m} \mathrm{t}\left(\mathcal{G}\left(F_{k}\right) ; G\right) .
$$

As in Lemma 2.14, ineq. (2.9) follows by considering the decomposition of $\mathcal{H}\left(C_{4}\right)$ into $F$ and each edge not in $E(F)$. 


\section{Chapter 3}

\section{Partitions, regularity and removal lemmas}

\subsection{Partition, projection and quotient}

An equipartition of a set $V$ is a partition $\left\{V_{i}\right\}_{i=1}^{k}$ of $V$ satisfying ||$V_{i}|-| V_{j} \| \leq 1$ for every $1 \leq i, j \leq k$. A partition/equipartition $\mathcal{V}$ of a weighted graph $\mathcal{G}_{\mathbb{R}}(V)$ is simply a partition/equipartition $\mathcal{V}=$ $\left\{V_{i}\right\}_{i=1}^{k}$ of its vertex set.

A partition $\mathcal{W}=\left\{W_{r}\right\}_{r=1}^{t}$ of $V$ is a refinement of a partition $\mathcal{V}=\left\{V_{i}\right\}_{i=1}^{k}$ of $V$ if for every $W_{r}$ there exists $V_{i}$ such that $W_{r} \subseteq V_{i}$. If this is the case, we write $\mathcal{V} \preceq \mathcal{W}$.

As an example, let $\mathcal{V}$ be a partition of $V$ and let $S \subseteq V$ and $\bar{S}=V \backslash S$. Then the partition $\mathcal{W}=$ $\left\{S \cap V_{i}\right\}_{i=1}^{k} \cup\left\{\bar{S} \cap V_{i}\right\}_{i=1}^{k}$ is a refinement of $\mathcal{V}$. We denote such partition $\mathcal{W}$ as $(\mathcal{V} \oplus S)$

Definition 3.1 (projection, $G_{\mathcal{V}}$ ). Given a weighted graph $G \in \mathcal{G}_{\mathbb{R}}(V)$ and a partition $\mathcal{V}=\left\{V_{i}\right\}_{i=1}^{k}$, we define the projection $G_{\mathcal{V}} \in \mathcal{G}_{\mathbb{R}}(V)$ of $G$ into $\mathcal{V}$ by putting

$$
G_{\mathcal{V}}(x, y)=\mathrm{d}\left(V_{i}, V_{j}\right) \text {, where } i, j \text { are indices for which } x \in V_{i}, y \in V_{j},
$$

for every $(x, y) \in V^{2}$. In other words $G_{\mathcal{V}}$ is defined by averaging $G$ over each of the cells $V_{i} \times V_{j}$. $\diamond$

The following proposition illustrates the use of the term "projection" for $\pi$.

Proposition 3.2. Let $\mathcal{V}=\left\{V_{i}\right\}_{i=1}^{k}$ be a partition of $G \in \mathcal{G}_{\mathbb{R}}(V)$. Then,

$$
\left\langle G-G_{\mathcal{V}}, G_{\mathcal{V}}\right\rangle=0
$$

In particular, we must have

$$
\|G\|_{2}^{2}=\|G \mathcal{V}\|_{2}^{2}+\|G-G \mathcal{V}\|_{2}^{2}
$$

Proof.

$$
\left\langle G-G_{\mathcal{V}}, G_{\mathcal{V}}\right\rangle=\frac{1}{n^{2}} \sum_{i j \in[k]^{2}} \sum_{x y \in V_{i} \times V_{j}}\left(G-G_{\mathcal{V}}(x, y)\right) G_{\mathcal{V}}(x, y) .
$$

But since $G_{\mathcal{V}}(x, y)=\mathrm{d}\left(V_{i}, V_{j}\right)$ for every $x y \in V_{i} \times V_{j}$, we get that

$$
\left\langle G-G_{\mathcal{V}}, G_{\mathcal{V}}\right\rangle=\sum_{i j \in[k]^{2}} \mathrm{~d}\left(V_{i}, V_{j}\right) \sum_{x y \in V_{i} \times V_{j}}\left(G(x, y)-\mathrm{d}\left(V_{i}, V_{j}\right)\right)=0,
$$


since $\sum_{x y \in\left(V_{i}, V_{j}\right)} G(x, y)=G\left(V_{i}, V_{j}\right)=\sum_{x y \in V_{i} \times V_{j}} \mathrm{~d}\left(V_{i}, V_{j}\right)$.

If $\mathcal{V}=\left\{V_{i}\right\}_{i=1}^{k}$ is an equipartition of a graph $G \in \mathcal{G}^{*}(V)$, every block $V_{i} \times V_{j}$ in the projection projection $G_{\mathcal{V}}$ has (essentially) the same size. In that case, the only information $G_{\mathcal{V}}$ carries is the value $\mathrm{d}\left(V_{i}, V_{j}\right)$ for each pair $i j \in[k]$, and thus we define the following graph in $\mathcal{G}([k])$ condensing this information.

Definition 3.3 (quotient, $G / \mathcal{V}$ ). Let $\mathcal{V}=\left\{V_{i}\right\}_{i=1}^{k}$ be an equipartition of a graph $G \in \mathcal{G}(V)$. Then, we define the quotient $G / \mathcal{V} \in \mathcal{G}^{*}([k])$ by putting

$G / \mathcal{V}$

$$
G / \mathcal{V}(i, j)=\mathrm{d}\left(V_{i}, V_{j}\right)
$$

for every pair $i j \in[k]^{2}$.

Despite the fact that equipartition classes do not have exactly the same size, the following simple proposition shows that, indeed, $G_{\mathcal{V}}$ and $G / \mathcal{V}$ carry essentially the same information regarding homomorphic densities of small graphs $F$.

Proposition 3.4. Let $\mathcal{V}=\left\{V_{i}\right\}_{i=1}^{k}$ be an equipartition of a graph $G \in \mathcal{G}(V)$ with $n=|V| \geq 2 k$. Then, for every graph $F=([f], E(F))$,

$$
\begin{aligned}
\mathrm{t}\left(F ; G_{\mathcal{V}}\right) & =\mathrm{t}(F ; G / \mathcal{V}) \pm \frac{2 k f}{n} \\
\mathrm{t}^{\bullet}\left(F ; G_{\mathcal{V}}\right) & =\mathrm{t}^{\bullet}(F ; G / \mathcal{V}) \pm \frac{2 k f}{n}
\end{aligned}
$$

Proof. For every $V_{i}$ we have $\left|V_{i}\right| /|V|=(n / k \pm 1) / n=\frac{1}{k}\left(1 \pm \frac{k}{n}\right)$. Hence, there is a function $w:[k]^{f} \rightarrow[0,1]$ for which we can write $\mathrm{t}\left(F ; G_{\mathcal{V}}\right)$ as

$$
\begin{aligned}
\mathrm{t}(F ; G \mathcal{V}) & =\sum_{\left(x_{1}, \ldots, x_{f}\right)} w\left(x_{1}, \ldots, x_{f}\right) \frac{\left|V_{x_{1}}\right|}{|V|} \ldots \frac{\left|V_{x_{f}}\right|}{|V|} \\
& =\sum_{\left(x_{1}, \ldots, x_{f}\right)} w\left(x_{1}, \ldots, x_{f}\right)\left(\frac{1}{k}\right)^{f}\left(1 \pm \frac{k}{n}\right)^{f} \\
& =\sum_{\left(x_{1}, \ldots, x_{f}\right)} w\left(x_{1}, \ldots, x_{f}\right)\left(\frac{1}{k}\right)^{f}\left(1 \pm \frac{2 k f}{n}\right) \\
& =\mathrm{t}(F ; G / \mathcal{V})\left(1 \pm \frac{2 k f}{n}\right)=\mathrm{t}(F ; G / \mathcal{V}) \pm \frac{2 k f}{n} .
\end{aligned}
$$

The proof for $\mathrm{t}^{\boldsymbol{\bullet}}\left(F ; G_{\mathcal{V}}\right)$ is identical.

Given a set $V$ and an integer $K \leq|V|$, we denote the set of all equipartitions of $V$ into $k$ classes by $\wp_{k}(V)$ and the set of all equipartitions of $V$ into at most $K$ classes by $\wp_{K}^{\leq}(V)=\bigcup_{k \leq K} \wp_{k}(V)$. $\wp_{K}, \wp_{K}^{\leqslant} \quad$ We also set $\wp(V)=\bigcup_{k<\infty} \wp_{k}(V)$.

For every set $\mathcal{S} \subseteq \wp(V)$ of equipartitions of $V$ and every graph $G \in \mathcal{G}(V)$, we define the set

$$
G / \mathcal{S}=\{\Gamma / \mathcal{V}: \mathcal{V} \in \mathcal{S}\}
$$

of all reduced graphs of $\Gamma$ with vertex size at most $K$. In particular $G / \wp_{K}^{\leqslant}(V)$ denotes the set of all quotients of $G$ by equipartitions of size at most $K$. 


\subsection{Regular and quad-regular pair}

Definition 3.5 (regular pair). A bipartite weighted graph $H \in \mathcal{H}_{[0,1]}(X, Y)$ is said to be $\gamma$-regular, for some $\gamma>0$, if

$$
\mathrm{d}(H[A, B])=\mathrm{d}(H) \pm \gamma \quad \text { for every } A \subseteq X, B \subseteq Y \text { with }|A| \geq \gamma|X| \text { and }|B| \geq \gamma|Y|
$$

Likewise, if $X, Y \subset V$ are (not necessarily disjoint) subsets of vertices of a graph $G \in \mathcal{G}^{*}(V)$, then we say that the pair $(X, Y)$ is $\gamma$-regular if

$$
\mathrm{d}(A, B)=\mathrm{d}(X, Y) \pm \gamma \quad \text { for every } A \subseteq X, B \subseteq Y \text { with }|A| \geq \gamma|X| \text { and }|B| \geq \gamma|Y| . \quad \diamond
$$

It is not difficult to see that the notion of regularity defined above is related to the cut norm of a bipartite graph.

Proposition 3.6. Let $H \in \mathcal{H}_{[0,1]}(X, Y)$ be a bipartite graph of density $d=\mathrm{d}(X, Y)$. If $(X, Y)$ is $\gamma$-regular, then $\|\Delta\|_{\square} \leq \gamma$, where $\Delta=H-d$.

Proof. Note that, since $\Delta$ is the weighted graph obtained by setting $\Delta(x, y)=H(x, y)-d$ for every $x \in X$ and $y \in Y$, we must have $\Delta \in \mathcal{H}_{[-1,1]}(X, Y)$. Let $A \subseteq X$ and $B \subseteq Y$ be fixed. We need to show that $|\Delta(A, B)| \leq \gamma|X||Y|$. If either $|A| \leq \gamma|X|$ or $|B| \leq \gamma|Y|$, then we trivially have $|\Delta(A, B)| \leq \gamma|X||Y|$. On the other hand, if both $|A| \geq \gamma|X|$ and $|B| \geq \gamma|Y|$, then

$$
|\Delta(A, B)|=|(d(A, B)-d)||A||B||\leq \gamma| A|| B|\leq \gamma| X|| Y \mid .
$$

It follows that $\|\Delta\|_{\square} \leq \gamma$.

In this thesis, it will be more convenient to work with the following less usual notion of regularity (see Chapters 5 and 6).

Definition 3.7 (quad-regular pair, $\operatorname{Reg}_{\text {gaco }_{0}}(X, Y)$ ). A bipartite weighted graph $H \in \mathcal{H}_{[0,1]}(X, Y)$ is said to be $\gamma$-quad-regular, for some $\gamma>0$, if

$$
\ddot{\mathrm{t}}_{C_{4}}(H) \leq \mathrm{d}(H)^{4}+\gamma \text {. }
$$

If $X, Y \subset V$ are (not necessarily disjoint) subsets of vertices of a weighted graph $G \in \mathcal{G}^{*}(V)$, then we say that $(X, Y)$ is $\gamma$-quad-regular if $G[X, Y]$ is $\gamma$-quad-regular, or, equivalently, if

$$
\ddot{\mathrm{t}}_{C_{4}}(X, Y) \leq \mathrm{d}(X, Y)^{4}+\gamma \text {. }
$$

We also set

$$
\operatorname{Reg}_{a_{a}}(X, Y)=\ddot{\mathrm{t}}_{C_{4}}(H)-\mathrm{d}(H)^{4}
$$

i.e., $\operatorname{Reg}_{\text {go }}(X, Y)$ is the minimum $\gamma$ for which $(X, Y)$ is $\gamma$-regular.

The next result asserts the notion of quad-regularity is, in some sense, equivalent to the more conventional notion of regularity defined in Definition 3.5.

Lemma 3.8. Let $H \in \mathcal{H}_{[0,1]}(X, Y)$ be a weighted bipartite graph. Then, 
(i) if $(X, Y)$ is $\frac{1}{8} \gamma^{8}$-quad-regular, then $(X, Y)$ is $\gamma$-regular;

(ii) if $(X, Y)$ is $\frac{1}{60} \gamma$-regular, then $(X, Y)$ is $\gamma$-quad-regular.

Proof. Let $d=d(X, Y)$ be the density of $H$ and let $\Delta=H-d$, i.e., $\Delta \in \mathcal{H}_{[-1,1]}(X, Y)$ is a bipartite weighted graph with $\Delta(x, y)=H(x, y)-d$ for every $x \in X, y \in Y$.

Proof of item (i): Let $(X, Y)$ be $\gamma$-quad-regular of density $d=\mathrm{d}(X, Y)$. Let $A \subseteq X$ and $B \subseteq Y$ with $|A| \geq \gamma|X|$ and $|B| \geq \gamma|Y|$ be given sets of density $d_{A, B}=\mathrm{d}(A, B)$.

We begin by showing that if $\ddot{\mathrm{t}}_{C_{4}}(\Delta)$ is small, then so is the deviation $\left|d_{A, B}-d\right|$ of the pair $(A, B)$.

$$
\begin{aligned}
|X|^{2}|Y|^{2} \ddot{\mathrm{t}}_{C_{4}}(\Delta) & =\sum_{\substack{x \in X \\
x^{\prime} \in X}} \sum_{\substack{y \in Y \\
y^{\prime} \in Y}} \Delta(x, y) \Delta\left(x, y^{\prime}\right) \Delta\left(x^{\prime}, y\right) \Delta\left(x^{\prime}, y^{\prime}\right) \\
& =\sum_{\substack{x \in X \\
x^{\prime} \in X}}\left(\sum_{y \in Y} \Delta(x, y) \Delta\left(x^{\prime}, y\right)\right)^{2} \geq \sum_{\substack{x \in A \\
x^{\prime} \in A}}\left(\sum_{y \in Y} \Delta(x, y) \Delta\left(x^{\prime}, y\right)\right)^{2} \\
& \geq|A|^{-2}\left(\sum_{\substack{x \in A \\
x^{\prime} \in A}} \sum_{y \in Y} \Delta(x, y) \Delta\left(x^{\prime}, y\right)\right)^{2} \\
& =|A|^{-2}\left(\sum_{y \in Y}\left(\sum_{x \in A} \Delta(x, y)\right)^{2}\right)^{2} \geq|A|^{-2}\left(\sum_{y \in B}\left(\sum_{x \in A} \Delta(x, y)\right)^{2}\right)^{2} \\
& \geq|A|^{-2}\left(|B|^{-1}\left(\sum_{y \in B} \sum_{x \in A} \Delta(x, y)\right)^{2}\right)^{2} \\
& =|A|^{-2}|B|^{-2} \Delta(A, B)^{4}=|A|^{2}|B|^{2}\left(d_{A, B}-d\right)^{4}
\end{aligned}
$$

Since $|A| \geq \gamma|X|$ and $|B| \geq \gamma|Y|$, we conclude that

$$
\ddot{\mathrm{t}}_{C_{4}}(\Delta) \geq \gamma^{4}\left(d_{A B}-d\right)^{4} \text {. }
$$

On the other hand, we can upper bound $\ddot{\mathrm{t}}_{C_{4}}(\Delta)$ as follows.

$$
\begin{aligned}
\ddot{\mathrm{t}}_{C_{4}}(\Delta) & =\ddot{\mathrm{t}}_{C_{4}}(H-d) \\
& =\mathbb{E}_{\varphi, \psi} \mathrm{hw}_{\varphi, \psi}\left(C_{4} ;(H-d)\right) \\
& =\mathbb{E}_{\varphi, \psi} \prod_{i j \in E\left(C_{4}\right)}(H(\varphi(i), \psi(j))-d) \\
& =\sum_{F \leq C_{4}} \mathbb{E}_{\varphi, \psi} \mathrm{hw}_{\psi, \varphi}(F ; H)(-d)^{4-\mathrm{e}(F)} \\
& =\sum_{F \leq C_{4}}(-1)^{\mathrm{e}(F)} \ddot{\mathrm{t}}_{F}(H) d^{4-\mathrm{e}(F)} .
\end{aligned}
$$

Note that, for every $F \leq C_{4}$, the term $\ddot{\mathrm{t}}_{F}(H) d^{4-\mathrm{e}(F)}$ in Eq. (3.3) is at most $\ddot{\mathrm{t}}_{C_{4}}(H)$ (by ineq. (2.3)) and at least $d^{4}$ (by the fact that Sidorenko's conjecture holds for all subgraphs of $C_{4}$ ). Hence, it follows from Eq. (3.3) that

$$
\ddot{\mathrm{t}}_{C_{4}}(\Delta) \leq \sum_{\substack{F \leq C_{4}: \\ \mathrm{e}(F) \text { even }}} \ddot{\mathrm{t}}_{C_{4}}(H)-\sum_{\substack{F \leq C_{4}: \\ \mathrm{e}(F) \text { odd }}} d^{4}=8\left(\ddot{\mathrm{t}}_{C_{4}}(H)-d^{4}\right) \leq \gamma^{8},
$$


since we started by assuming $\ddot{\mathrm{t}}_{C_{4}}(H)-d^{4} \leq \frac{1}{8} \gamma^{8}$. By combining ineq. (3.2) and (3.4), we conclude that $\left(d_{A B}-d\right)^{4} \leq \gamma^{4}$, i.e., that $d_{A B}=d \pm \gamma$. Therefore, $(X, Y)$ is a $\gamma$-regular pair.

Proof of item (ii): For the second item, first note that since $(X, Y)$ is $\frac{1}{60} \gamma$-regular then $\|\Delta\|_{\square} \leq \frac{1}{60} \gamma$ by Proposition 3.6. Moreover, for every fixed $x^{\prime} \in X$ and $y^{\prime} \in Y$, define the functions $f_{x^{\prime}, y^{\prime}}(x)=$ $\Delta\left(x, y^{\prime}\right) \Delta\left(x^{\prime}, y^{\prime}\right)$ and $g_{x^{\prime}, y^{\prime}}(y)=\Delta\left(x^{\prime}, y\right)$ for every $x \in X, y \in Y$. Then, note that

$$
\begin{aligned}
\ddot{\mathrm{t}}_{C_{4}}(\Delta) & =\frac{1}{|X|^{2}|Y|^{2}} \sum_{\substack{x^{\prime} \in X \\
y^{\prime} \in Y}} \sum_{\substack{x \in X \\
y \in Y}} \Delta\left(x, y^{\prime}\right) \Delta\left(x^{\prime}, y^{\prime}\right) \Delta(x, y) \Delta\left(x^{\prime}, y\right) \\
& =\frac{1}{|X|^{2}|Y|^{2}} \sum_{\substack{x^{\prime} \in X \\
y^{\prime} \in Y}} \sum_{\substack{x \in X \\
y \in Y}} f_{x^{\prime}, y^{\prime}}(x) \cdot \Delta(x, y) \cdot g_{x^{\prime}, y^{\prime}}(y) \\
& \leq \frac{1}{|X||Y|} \sum_{\substack{x^{\prime} \in X \\
y^{\prime} \in Y}}\|\Delta\|_{\square_{f, g}}=\|\Delta\|_{\square_{f, g}},
\end{aligned}
$$

where $\|\Delta\|_{\square_{f, g}}$ is as in Remark 2.12 .

Finally, by proceeding analogously to Eq. (3.3), we can write

$$
\begin{aligned}
\ddot{\mathrm{t}}_{C_{4}}(H)=\ddot{\mathrm{t}}_{C_{4}}(d+\Delta)=\sum_{F \leq C_{4}} d^{4-\mathrm{e}(F)} \ddot{\mathrm{t}}_{F}(\Delta)=d^{4}+\sum_{\substack{F \leq C_{4} \\
\mathrm{e}(F)>0}} d^{4-\mathrm{e}(F)} \ddot{\mathrm{t}}_{F}(\Delta) \\
\quad \stackrel{(2.3)}{\leq} d^{4}+15 \ddot{\mathrm{t}}_{C_{4}}(\Delta) \leq d^{4}+15\|\Delta\|_{\square_{f, g}} \stackrel{(2.13)}{\leq} d^{4}+60\|\Delta\|_{\square} \leq d^{4}+\gamma,
\end{aligned}
$$

which implies the pair $(X, Y)$ is $\gamma$-quad-regular.

For a different proof of the second item of Lemma 3.8 above, which also provides a better constant, see Corollary 3.25.

The following result from $\left[\mathrm{BCL}^{+}\right.$08, Theorem 2.10] (see also [RV07, Corollary 1.7], [AFdlVKK03, Theorem 6]), states that if the cut norm of a weighted graph $\Delta$ is bounded, then so is the cut norm of a typical sample of $\Delta$.

Theorem 3.9. For every $\gamma>0$ and $\delta>0$, there is $\gamma^{\prime}=\operatorname{poly}(\gamma)$ and a positive integer $q=$ poly $\left(\gamma^{-1} \log \delta^{-1}\right)$ such that if $\Delta \in[-1,1]^{V \times V}$ is a weighted graph satisfying

$$
\|\Delta\|_{\square} \leq \gamma^{\prime}
$$

and $Q \in_{U}\left(\begin{array}{l}V \\ q\end{array}\right)$, then

$$
\|\Delta[Q]\|_{\square} \leq \gamma
$$

with probability at least $1-\delta$.

\subsection{Frieze-Kannan regularity}

Lemma 3.10. Let $\mathcal{V}=\left\{V_{i}\right\}_{i=1}^{k}$ be a partition of a graph $G \in \mathcal{G}_{\mathbb{R}}(V)$. For any given $S \subseteq V$,

$$
\left\|G_{\mathcal{W}}\right\|_{2}^{2}-\left\|G_{\mathcal{V}}\right\|_{2}^{2} \geq\left(\frac{1}{n^{2}} \Delta(S, S)\right)^{2},
$$


where $\Delta=G-G \mathcal{V}$ and $\mathcal{W}=\mathcal{V} \oplus S$.

Proof. For every $i \in[k]$, let $S_{i}=S \cap V_{i}$. Since, by (3.1), the left hand side of ineq. (3.8) is equal to $\left\|G_{\mathcal{V}}-G_{\mathcal{W}}\right\|_{2}^{2}$, we get

$$
\begin{aligned}
\left\|G_{\mathcal{W}}\right\|_{2}^{2}-\left\|G_{\mathcal{V}}\right\|_{2}^{2} & =\frac{1}{n^{2}} \sum_{x y \in V^{2}}\left(G_{\mathcal{W}}(x, y)-G_{\mathcal{V}}(x, y)\right)^{2} \\
& \geq\left(\frac{1}{n^{2}} \sum_{x y \in V^{2}}\left|G_{\mathcal{W}}(x, y)-G_{\mathcal{V}}(x, y)\right|\right)^{2} \\
& \geq\left(\frac{1}{n^{2}} \sum_{i j \in[k]^{2}} \sum_{x y \in\left(S_{i}, S_{j}\right)}\left|G_{\mathcal{W}}(x, y)-G_{\mathcal{V}}(x, y)\right|\right)^{2} \\
& \geq\left(\frac{1}{n^{2}} \sum_{i j \in[k]^{2}}\left|G_{\mathcal{W}}\left(S_{i}, S_{j}\right)-G_{\mathcal{V}}\left(S_{i}, S_{j}\right)\right|\right)^{2} \\
& =\left(\frac{1}{n^{2}} \sum_{i j \in[k]^{2}}\left|G\left(S_{i}, S_{j}\right)-G_{\mathcal{V}}\left(S_{i}, S_{j}\right)\right|\right)^{2} \\
& =\left(\frac{1}{n^{2}} \sum_{i j \in[k]^{2}}\left|\Delta\left(S_{i}, S_{j}\right)\right|\right)^{2} \\
& \geq\left(\frac{1}{n^{2}}|\Delta(S, S)|\right)^{2} .
\end{aligned}
$$

Definition 3.11 (FK-regular partition). Given $\gamma>0$ and a graph $G=(V, E)$, a partition $\mathcal{V}=$ $\left\{V_{i}\right\}_{i=1}^{k}$ of $V$ is called FK-regular if

$$
\left\|G-G_{\mathcal{V}}\right\|_{\square} \leq \gamma
$$

or, equivalently, if

$$
\left|G(A, B)-\sum_{i j \in[k]^{2}} \mathrm{~d}\left(V_{i}, V_{j}\right)\right| A \cap V_{i}|| B \cap V_{j}|| \leq \gamma|V|^{2},
$$

for every $A, B \subseteq V$.

Lemma 3.12 ([FK99]). For every $\gamma>0$, there is an integer $T=T_{3.12}=\operatorname{poly}\left(\gamma^{-1}\right)$ satisfying the following. For every graph $G=(V, E)$, and every partition $\mathcal{V}_{1}$ of $G$ into $k_{1}$ classes, there is a partition $\mathcal{V}=\left\{V_{i}\right\}_{i=1}^{k}$ of $G$, into $k_{1} \leq k \leq k_{1} \cdot 2^{T}$ classes, for which $\mathcal{V}$ is $\gamma$-FK-regular. Moreover, $\mathcal{V}$ refines the given partition $\mathcal{V}_{1}$.

Proof. Let $T=\left\lceil 4 \gamma^{-2}\right\rceil+1$. Consider partitions $\left\{\mathcal{V}_{t}\right\}_{t=1}^{T}$ obtained in the following way:

(i) $\mathcal{V}_{1}$ is as given by the statement of the lemma.

(ii) $\mathcal{V}_{t}=\mathcal{V}_{t-1} \oplus S_{t}$, where $S_{t} \subseteq V$ is a set that maximizes $\left|\left(G-G \mathcal{V}_{t-1}\right)(S, S)\right|$ (for every $t \geq 2$ )

Let $G_{t}=G_{\mathcal{V}_{t}}$ and note that $G_{t-1}=\left(G_{t}\right)_{\mathcal{V}_{t-1}}$ for every $t \geq 2$. Hence,

$$
0 \leq\left\|G_{1}\right\|_{2}^{2} \leq\left\|G_{2}\right\|_{2}^{2} \leq \cdots \leq\left\|G_{T}\right\|_{2}^{2} \leq 1
$$

By the pigeonhole principle, there must be some $2 \leq t \leq T$ for which

$$
\left\|G_{t}\right\|_{2}^{2}-\left\|G_{t-1}\right\|_{2}^{2} \leq \frac{1}{T-1} \leq \frac{1}{4} \gamma^{2} .
$$


Lemma 3.10 asserts that we should have

$$
\frac{1}{4} \gamma^{2} \geq\left\|G_{t}\right\|_{2}^{2}-\left\|G_{t-1}\right\|_{2}^{2} \stackrel{(3.10)}{\geq}\left(\frac{1}{n^{2}}\left(G-G_{t-1}\right)\left(S_{t}, S_{t}\right)\right)^{2} \geq \frac{1}{4}\left\|\left(G-G \mathcal{V}_{t-1}\right)\right\|_{\square}^{2},
$$

where the last inequality comes from the choice of $S_{t}$ and Proposition 2.10. Therefore $\mathcal{V}=\mathcal{V}_{t-1}$ is a partition as required.

The bound for the number of classes given by Lemma 3.12 is close to best possible, since Conlon and Fox [CF12] found graph instances where the number of classes in any $\gamma$-FK-regular partition is at least $k \geq 2^{1 /\left(2^{60} \gamma^{2}\right)}$ (for a previous result, see Lovász and Szegedy [LS07]).

Given a real number $d$ and two (not necessarily disjoint) sets $X, Y \subseteq V$, we define the cut $d K_{X, Y}$ as the matrix $M \in \mathbb{R}^{V \times V}$ satisfying $M(x, y)=d$ if $(x, y) \in(X, Y)$, and $M(x, y)=0$ otherwise (the underlying set $V$ will always be clear from context). The following lemma, stated for graphs, was originally proven in [FK99] for arbitrary matrices. It shows that every graph can be approximated (with respect to the cut norm) by a small sum of cuts.

Lemma 3.13 (Cut Decomposition [FK99, Theorem 2]). For every $\gamma>0$, there is an integer $T=$ $T_{3.13}=\operatorname{poly}\left(\gamma^{-1}\right)$ satisfying the following. For every graph $G=(V, E)$, with $|V| \geq T$, there are sets $X_{1}, \ldots, X_{t}, Y_{1}, \ldots, Y_{t} \subseteq V$ and real numbers $d_{1}, \ldots, d_{t}$ with $t \leq T$ for which

$$
G=\sum_{r=1}^{t} d_{r} K_{X_{r}, Y_{r}}+\Delta,
$$

where the "error" weighted-graph $\Delta \in \mathcal{G}_{\mathbb{R}}(V)$ satisfies $\|\Delta\|_{\square} \leq \gamma$. Moreover, there is an absolute constant $D_{3.13}$ for which $\sum_{r=1}^{t} d_{r}^{2} \leq D_{3.13}$.

We will actually use (in Chapter 5) the following slightly simplified version of Lemma 3.13, which approximates the graph as sums of "symmetric" cuts. Given a set $S \subseteq V$ and $d \in \mathbb{R}$, we define the symmetric cut $d K_{S}:=d K_{S, S}$.

Lemma 3.14 (Cut Decomposition (symmetric version)). For every $\gamma>0$, there is an integer $T=$ $T_{3.14}=\operatorname{poly}\left(\gamma^{-1}\right)$ satisfying the following. For every graph $G=(V, E)$, with $|V| \geq T$, there are sets $S_{1}, \ldots, S_{t} \subseteq V$ and real numbers $d_{1}, \ldots, d_{t}$ with $t \leq T$ for which

$$
G=\sum_{r=1}^{t} d_{r} K_{S_{r}}+\Delta
$$

where the "error" weighted-graph $\Delta \in \mathcal{G}_{\mathbb{R}}(V)$ satisfies $\|\Delta\|_{\square} \leq \gamma$. Moreover, there is an absolute constant $D_{3.14}$ for which $\sum_{r=1}^{t} d_{r}^{2} \leq D_{3.14}$.

Proof. We will show one can take $D_{3.14}=D_{3.13}$ and $T_{3.14}(\gamma)=4 T_{3.13}(\gamma)$ for any given $\gamma>0$. Indeed, for every graph $G=(V, E)$ with $|V| \geq T$, apply Lemma 3.13 to $G$ to get an integer $t \leq T$, sets $X_{1}, \ldots, X_{t}, Y_{1}, \ldots, Y_{t} \subseteq V$ and numbers $d_{1}, \ldots, d_{t}$ satisfying

$$
G=\sum_{r=1}^{t} d_{r} K_{X_{r}, Y_{r}}+\Delta,
$$

with $\|\Delta\|_{\square} \leq \gamma$. Since a (weighted) graph is, in particular, a matrix in $\{0,1\}^{V \times V}$ (as formally 
defined in Chapter 2), we can transpose each side of Eq. (3.11) above to get

$$
G^{T}=\sum_{r=1}^{t} d_{r} K_{Y_{r}, X_{r}}+\Delta^{T}
$$

By summing Eqs. (3.11) and (3.12) and dividing by two, we get

$$
G=\frac{1}{2}\left(G+G^{T}\right)=\sum_{r=1}^{t} \frac{1}{2} d_{r}\left(K_{X_{r}, Y_{r}}+K_{Y_{r}, X_{r}}\right)+\Delta .
$$

By applying the inclusion-exclusion principle, one can rewrite the $r$-th term (for every $1 \leq r \leq t$ ) on the right side of Eq. (3.13) as

$$
\frac{1}{2} d_{r}\left(K_{X_{r}, Y_{r}}+K_{Y_{r}, X_{r}}\right)=\frac{1}{2} d_{r}\left(K_{X_{r} \cup Y_{r}}-K_{X_{r} \backslash Y_{r}}-K_{Y_{r} \backslash X_{r}}+K_{X_{r} \cap Y_{r}}\right) .
$$

After replacing each of these terms in Eq. (3.13), we get

$$
G=\sum_{r^{\prime}=1}^{4 t} d_{r^{\prime}}^{\prime} K_{S_{r^{\prime}}}+\Delta
$$

where $d_{4 r-3}^{\prime}=\frac{1}{2} d_{r}$ and $S_{4 r-3}=X_{r} \cup Y_{r}, d_{4 r-2}^{\prime}=-\frac{1}{2} d_{r}$ and $S_{4 r-2}=X_{r} \backslash Y_{r}, d_{4 r-1}^{\prime}=-\frac{1}{2} d_{r}$ and $S_{4 r-1}=Y_{r} \backslash X_{r}, d_{4 r}^{\prime}=\frac{1}{2} d_{r}$ and $S_{4 r}=X_{r} \cap Y_{r}$ (for every $1 \leq r \leq t$ ). Finally, we note that

$$
\sum_{r^{\prime}=1}^{4 t}{d_{r^{\prime}}^{\prime}}^{2}=4 \sum_{r=1}^{t}\left(\frac{1}{2} d_{r}\right)^{2}=\sum_{r=1}^{t} d_{r}^{2} \leq D_{3.13},
$$

as required.

The concept of $F K$-regularity is also known as weak regularity. The following lemma is also a consequence of Lemma 3.12 - since it is not difficult to see one can turn an $\gamma^{\prime}$-FK-regular partition FK-regular equipartition (without significantly increasing $\gamma^{\prime}$ or the size of the partition)

Lemma 3.15 (Frieze-Kannan Regularity Lemma). For every $\gamma>0$ and every $k_{0}>0$, there is $K=k_{0} \cdot 2^{\mathrm{poly}(1 / \gamma)}$ such that every graph $\Gamma$ on $n \geq K$ vertices admits a $\gamma$-FK-regular equipartition into $k$ classes, where $k_{0} \leq k \leq K$.

As mentioned before, Conlon and Fox [CF12] found graphs where the number of classes in any $\gamma$-FK-regular partition (in particular, any equipartition) is at least $2^{1 /\left(2^{60} \gamma^{2}\right)}$.

\subsection{Szemerédi regularity and variants}

For every $\gamma>0$, an equipartition $\mathcal{V}=\left\{V_{i}\right\}_{i=1}^{k}$ of a graph $G$ is $\gamma$-regular if $\left(V_{i}, V_{j}\right)$ is $\gamma$-regular for all but at most $\gamma k^{2}$ of the pairs $i j \in[k]^{2}$.

Lemma 3.16 (Szemerédi's Regularity Lemma [Sze78]). For every $\gamma>0$, there is $K=K_{3.16}(\gamma)=$ $\operatorname{TOWER}\left(\operatorname{poly}\left(\gamma^{-1}\right)\right)$ satisfying the following. For every graph $G$ there is a $\gamma$-regular equipartition $\left\{V_{i}\right\}_{i=1}^{k}$, with $k \leq K$. 
Gowers [Gow97] showed that there are graphs for which there are no $\gamma$-regular equipartitions $\left\{V_{i}\right\}_{i=1}^{k}$, with $k<F(\gamma)$, with $F(\gamma)=\operatorname{TOWER}\left(\operatorname{poly}\left(\gamma^{-1}\right)\right)$.

Alon, Fischer, Szegedy, Krivelevich proved the stronger version of regularity stated below in $[\mathrm{AFKS00]}$.

Lemma 3.17 (Strong Regularity Lemma). For every decreasing function $\mathcal{E}: \mathbb{N} \rightarrow(0,1]$, there is $K=K_{3.17}(\mathcal{E})$ satisfying the following. For every graph $G$, there is an equipartition $\mathcal{V}=\left\{V_{i}\right\}_{i=1}^{k}$ and a refined equipartition $\mathcal{W}=\left\{V_{i, j}\right\}_{i \in[k]}^{j \in[\ell]}$, with $V_{i, j} \subseteq V_{i}$ for every $j \in[\ell]$, satisfying the following

(i) $k \ell \leq K$

(ii) $\mathcal{V}$ is $\mathcal{E}(0)$-regular;

(iii) $\mathcal{W}$ is $\mathcal{E}(k)$-regular;

(iv) all but at most $\mathcal{E}(0) k^{2}$ of the pairs $i i^{\prime} \in[k]^{2}$ are such that: $\mathrm{d}\left(V_{i}, V_{i^{\prime}}\right)=\mathrm{d}\left(V_{i, j}, V_{i^{\prime}, j^{\prime}}\right) \pm \mathcal{E}(0)$ for all but at most $\mathcal{E}(0) \ell^{2}$ of the pairs $j j^{\prime}$.

Moreover, $K_{3.17}(\mathcal{E}) \leq F^{\left(\left\lceil\operatorname{poly}\left(\mathcal{E}(0)^{-1}\right)\right\rceil\right)}(1)$, where $F(t) \leq \operatorname{TOWER}(t / \mathcal{E}(t))$.

Note that even for a constant function $\mathcal{E}(t)=\gamma$ for every $t \in \mathbb{N}$, the upper bound to $K_{3.17}$ is a WOWZER $\left(\operatorname{poly}\left(\gamma^{-1}\right)\right)$. Conlon and Fox [CF12] showed there are graphs $G$ that require such a bound.

Most applications of Lemma 3.17, like the induced graph removal lemma, makes use of the following corollary - which can be easily obtained by considering the equipartitions $\mathcal{V}$ and $\mathcal{W}$ from Lemma 3.17 and choosing a class from $\left\{V_{i, j}\right\}_{j \in[\ell]}$ (for each $i \in[k]$ ) uniformly at random.

Lemma 3.18 (Strong regularity key corollary). For every decreasing function $\mathcal{E}: \mathbb{N} \rightarrow(0,1]$, there is $\delta=\delta_{3.18}(\mathcal{E})$, such that every graph $G=(V, E)$ with $|V| \geq \delta^{-1}$ has an equipartition $\left\{V_{i}\right\}_{i=1}^{k}$ with each $V_{i}$ containing a set $W_{i}$ of size $\left|W_{i}\right| \geq \delta|V|$ satisfying the following:

(i) every pair $\left(W_{i}, W_{j}\right)$ is $\mathcal{E}(k)$-regular $(1 \leq i, j \leq k)$;

(ii) all but at most $\mathcal{E}(0) k^{2}$ pairs $1 \leq i \leq j \leq k$ satisfy $\mathrm{d}\left(V_{i}, V_{j}\right)=\mathrm{d}\left(W_{i}, W_{j}\right) \pm \mathcal{E}(0)$.

Conlon and Fox showed in [CF12] that one can obtain Lemma 3.18 by a different method and get a better upper bound for $\delta^{-1}$. More precisely, they showed $\delta_{3.17}(\mathcal{E})^{-1} \leq F^{\left(\left\lceil\operatorname{poly}\left(\mathcal{E}(0)^{-1}\right)\right\rceil\right)}(1)$, where $F(t)=\exp \{t / \mathcal{E}(t)\}$. In particular, one gets a bound of $\delta^{-1}=\operatorname{TOWER}\left(\operatorname{poly}\left(\gamma^{-1}\right)\right)$ for $\mathcal{E}(t)=\gamma$.

\subsection{Counting Lemmas}

In this section, we presult results allowing one to approximate the homomorphic density (both induced or not) of copies of a small graph $F$ in $G$ based on an FK-regular partition of $G$. Such results are generally called (global) counting lemma results.

In what follows, we will need the following simple fact.

Fact 3.19. Let $a_{1}, \ldots, a_{t}$ and $b_{1}, \ldots, b_{t}$ be real numbers. Then

$$
\prod_{i=1}^{t} a_{i}-\prod_{i=1}^{t} b_{i}=\sum_{j=1}^{t} \prod_{i<j} a_{i} \cdot\left(a_{j}-b_{j}\right) \cdot \prod_{i>j} b_{i} .
$$


Proof. Observe that

$$
\sum_{j=1}^{t} \prod_{i<j} a_{i} \cdot\left(a_{j}-b_{j}\right) \cdot \prod_{i>j} b_{i}=\sum_{j=1}^{t} \prod_{i \leq j} a_{i} \prod_{i>j} b_{i}-\sum_{j=1}^{t} \prod_{i<j} a_{i} \prod_{i \geq j} b_{i}=\prod_{i=1}^{t} a_{i}-\prod_{i=1}^{t} b_{i},
$$

which is the desired result.

Lemma 3.20 (see [Lov12, Theorem 10.24]). Let $G_{1} \in \mathcal{G}^{*}(V)$ and $G_{2} \in \mathcal{G}^{*}(V)$ be two weighted graphs over $V$ and let $F=([f], E(F))$ be a simple graph. Then

$$
\left|\mathrm{t}^{\bullet}\left(F ; G_{1}\right)-\mathrm{t}^{\bullet}\left(F ; G_{2}\right)\right| \leq\left(\begin{array}{c}
f \\
2
\end{array}\right)\left\|G_{1}-G_{2}\right\|_{\square} .
$$

Proof. For every pair $u v \in\left(\begin{array}{c}{[f]} \\ 2\end{array}\right)$, any vertices $x, y \in V$ and $i \in\{1,2\}$, define

$$
g_{i}^{(u, v)}(x, y)= \begin{cases}G_{i}(x, y) & \text { if } u v \in E(F) \\ 1-G_{i}(x, y) & \text { if } u v \notin E(F)\end{cases}
$$

We have

$$
n^{f}\left(\mathrm{t}^{\bullet}\left(F ; G_{1}\right)-\mathrm{t}^{\bullet}\left(F ; G_{2}\right)\right)=\sum_{\left(x_{1}, \ldots, x_{f}\right)}\left(\prod_{u v \in\left(\begin{array}{c}
{[f]} \\
2
\end{array}\right)} g_{1}^{(u, v)}\left(x_{u}, x_{v}\right)-\prod_{u v \in\left(\begin{array}{c}
{[f]} \\
2
\end{array}\right)} g_{2}^{(u, v)}\left(x_{u}, x_{v}\right)\right),
$$

where the sum is over all $n^{f}$ sequences of $f$ vertices in $V$. We consider an arbitrary linear ordering < of the elements $u v \in\left(\begin{array}{c}{[f]} \\ 2\end{array}\right)$, and apply Fact 3.19 to get

$$
\begin{aligned}
& n^{f}\left(\mathrm{t}^{\boldsymbol{\bullet}}\left(F ; G_{1}\right)-\mathrm{t}^{\boldsymbol{\Theta}}\left(F ; G_{2}\right)\right) \\
& =\sum_{\left(x_{1}, \ldots, x_{f}\right)} \sum_{u v \in\left(\begin{array}{c}
{[f]} \\
2
\end{array}\right)}\left(\prod_{a b<u v} g_{1}^{(a, b)}\left(x_{a}, x_{b}\right)\right) \cdot\left(g_{1}^{(u, v)}\left(x_{u}, x_{v}\right)-g_{2}^{(u, v)}\left(x_{u}, x_{v}\right)\right) \cdot\left(\prod_{a b>u v} g_{2}^{(a, b)}\left(x_{a}, x_{b}\right)\right) \\
& =\sum_{u v \in\left(\begin{array}{c}
{[f]} \\
2
\end{array}\right)} \sum_{\left(x_{1}, \ldots, x_{f}\right)}\left(\prod_{a b<u v} g_{1}^{(a, b)}\left(x_{a}, x_{b}\right)\right) \cdot\left(g_{1}^{(u, v)}\left(x_{u}, x_{v}\right)-g_{2}^{(u, v)}\left(x_{u}, x_{v}\right)\right) \cdot\left(\prod_{a b>u v} g_{2}^{(a, b)}\left(x_{a}, x_{b}\right)\right) .
\end{aligned}
$$

For every $u v \in\left(\begin{array}{c}{[f]} \\ 2\end{array}\right)$, one can rewrite the summation over $\left(x_{1}, \ldots, x_{f}\right)$ so that the vertices $x_{u}$ and $x_{v}$ are "chosen" after the remaining vertices $\vec{x}:=\left\{\left(x_{w}\right)\right\}_{w \neq u, v}$, as follows:

$$
\begin{aligned}
& n^{f}\left(\mathrm{t}^{\boldsymbol{\Theta}}\left(F ; G_{1}\right)-\mathrm{t}^{\boldsymbol{\Theta}}\left(F ; G_{2}\right)\right) \\
& =\sum_{u v \in\left(\begin{array}{c}
{[f]} \\
2
\end{array}\right)} \sum_{\vec{x}} \sum_{x_{u}, x_{v}}\left(\prod_{a b<u v} g_{1}^{(a, b)}\left(x_{a}, x_{b}\right)\right) \cdot\left(g_{1}^{(u, v)}\left(x_{u}, x_{v}\right)-g_{2}^{(u, v)}\left(x_{u}, x_{v}\right)\right) \cdot\left(\prod_{a b>u v} g_{2}^{(a, b)}\left(x_{a}, x_{b}\right)\right),
\end{aligned}
$$

where the sum $\sum_{\vec{x}}$ is over all sequences $\vec{x}=\left(x_{w}\right)_{w \in[f] \backslash\{u, v\}}$ of $f-2$ vertices of $V$, indexed by vertices $w \in[f]$ with $w \neq u, v$.

Next, let $u v \in\left(\begin{array}{c}{[f]} \\ 2\end{array}\right)$ and $\vec{x}$ be fixed. Let $T(v)=\left\{a b \in\left(\begin{array}{c}{[f]} \\ 2\end{array}\right):\{a, b\} \cap\{v\} \neq \emptyset\right\}$ be the set of pairs $a b$ that touch $v$. Then we can write

$$
\prod_{a b<u v} g_{1}^{(a, b)}\left(x_{a}, x_{b}\right) \cdot \prod_{a b>u v} g_{2}^{(a, b)}\left(x_{a}, x_{b}\right)=\alpha^{\vec{x}}\left(x_{u}\right) \cdot \beta^{\vec{x}}\left(x_{v}\right)
$$


where the functions $\alpha^{\vec{x}}, \beta^{\vec{x}}: V \rightarrow[0,1]$ are defined as follows:

$$
\begin{aligned}
\alpha^{\vec{x}}\left(x_{u}\right) & =\prod_{\substack{a b<u v \\
a b \notin T(v)}} g_{1}^{(a, b)}\left(x_{a}, x_{b}\right) \cdot \prod_{\substack{a b>u v \\
a b \notin T(v)}} g_{2}^{(a, b)}\left(x_{a}, x_{b}\right) ; \\
\beta^{\vec{x}}\left(x_{v}\right) & =\prod_{\substack{a b<u v \\
a b \in T(v)}} g_{1}^{(a, b)}\left(x_{a}, x_{b}\right) \cdot \prod_{\substack{a b>u v \\
a b \in T(v)}} g_{2}^{(a, b)}\left(x_{a}, x_{b}\right) .
\end{aligned}
$$

Indeed, $\alpha^{\vec{x}}\left(x_{u}\right)$ clearly does not depend on $x_{v}$ and $\beta^{\vec{x}}\left(x_{v}\right)$ does not depend on $x_{u}$ since otherwise we would have $\{a, b\}=\{u, v\}$ for some term in the definition of $\beta^{\vec{x}}$.

Hence, by setting $\Delta=G_{1}-G_{2}$, we have

$$
\begin{aligned}
n^{f}\left|\mathrm{t}^{\boldsymbol{\Theta}}\left(F ; G_{1}\right)-\mathrm{t}^{\boldsymbol{\Theta}}\left(F ; G_{2}\right)\right| \leq & \sum_{u v \in\left(\begin{array}{c}
{[f]} \\
2
\end{array}\right)} \sum_{\vec{x}} \sum_{x_{u}, x_{v}}\left(\alpha^{\vec{x}}\left(x_{u}\right)\left(g_{1}^{(u, v)}\left(x_{u}, x_{v}\right)-g_{2}^{(u, v)}\left(x_{u}, x_{v}\right)\right) \beta^{\vec{x}}\left(x_{v}\right)\right) \mid \\
\leq & \left|\sum_{u v \in E(F)} \sum_{\vec{x}} \sum_{x_{u}, x_{v}}\left(\alpha^{\vec{x}}\left(x_{u}\right) \Delta\left(x_{u}, x_{v}\right) \beta^{\vec{x}}\left(x_{v}\right)\right)\right| \\
& +\left|\sum_{u v \notin E(F)} \sum_{\vec{x}} \sum_{x_{u}, x_{v}}\left(\alpha^{\vec{x}}\left(x_{u}\right)\left(-\Delta\left(x_{u}, x_{v}\right)\right) \beta^{\vec{x}}\left(x_{v}\right)\right)\right| .
\end{aligned}
$$

By Lemma 2.13, the absolute value of each of the sums over $x_{u}, x_{v}$ can be bounded by $\|\Delta\|_{\square} n^{2}$. By doing so, we get that

$$
\begin{aligned}
\left|\mathrm{t}^{\bullet}\left(F ; G_{1}\right)-\mathrm{t}^{\bullet}\left(F ; G_{2}\right)\right| & \leq \frac{1}{n^{f}}\left(|E(F)| \cdot n^{f-2} \cdot\|\Delta\|_{\square} n^{2}+\left(\left(\begin{array}{l}
f \\
2
\end{array}\right)-|E(F)|\right) \cdot n^{f-2} \cdot\|\Delta\|_{\square} n^{2}\right) \\
& =\left(\begin{array}{l}
f \\
2
\end{array}\right)\|\Delta\|_{\square},
\end{aligned}
$$

as required.

Lemma 3.21. Let $G_{1} \in \mathcal{G}^{*}(V)$ and $G_{2} \in \mathcal{G}^{*}(V)$ be two weighted graphs over $V$ and let $F=$ $([f], E(F))$ be a simple graph. Then

$$
\left|\mathrm{t}\left(F ; G_{1}\right)-\mathrm{t}\left(F ; G_{2}\right)\right| \leq \mathrm{e}(F)\left\|G_{1}-G_{2}\right\|_{\square} .
$$

Proof. Let $\Delta=G_{1}-G_{2}$. For every pair $u v \in\left(\begin{array}{c}{[f]} \\ 2\end{array}\right)$, any vertices $x, y \in V$ and $i \in\{1,2\}$, define

$$
g_{i}^{(u, v)}(x, y)= \begin{cases}G_{i}(x, y) & \text { if } u v \in E(F) \\ 1 & \text { if } u v \notin E(F)\end{cases}
$$

The proof follows exactly the proof of Lemma 3.20, except that in Eq. (3.15) we now have $g_{1}^{(u, v)}\left(x_{u}, x_{v}\right)-g_{2}^{(u, v)}\left(x_{u}, x_{v}\right)=0$ for $u v \notin E(F)$. Hence, we get that

$$
n^{f}\left|\mathrm{t}\left(F ; G_{1}\right)-\mathrm{t}\left(F ; G_{2}\right)\right| \leq\left|\sum_{u v \in\left(\begin{array}{c}
{[f]} \\
2
\end{array}\right)} \sum_{\vec{x}} \sum_{x_{u}, x_{v}}\left(\alpha^{\vec{x}}\left(x_{u}\right)\left(g_{1}^{(u, v)}\left(x_{u}, x_{v}\right)-g_{2}^{(u, v)}\left(x_{u}, x_{v}\right)\right) \beta^{\vec{x}}\left(x_{v}\right)\right)\right|
$$




$$
\leq\left|\sum_{u v \in E(F)} \sum_{\vec{x}} \sum_{x_{u}, x_{v}}\left(\alpha^{\vec{x}}\left(x_{u}\right) \Delta\left(x_{u}, x_{v}\right) \beta^{\vec{x}}\left(x_{v}\right)\right)\right| .
$$

As in the proof of Lemma 3.20, we can again use Lemma 2.13 to bound each of the sums over $x_{u}, x_{v}$ by $\|\Delta\|_{\square} n^{2}$. By doing so, we get that

$$
\left|\mathrm{t}\left(F ; G_{1}\right)-\mathrm{t}\left(F ; G_{2}\right)\right| \leq \frac{1}{n^{f}}\left(|E(F)| \cdot n^{f-2} \cdot\|\Delta\|_{\square} \cdot n^{2}\right)=\mathrm{e}(F)\|\Delta\|_{\square},
$$

as required.

Lemma 3.22 (see [Lov12, Theorem 10.24]). Let $\mathcal{V}$ be a $\gamma$-FK-regular equipartition of a graph $G \in \mathcal{G}$. Then, for every graph $F=([f], E(F)) \in \mathcal{G}$ we have

$$
\mathrm{t}^{\boldsymbol{\bullet}}(F ; G)=\mathrm{t}^{\boldsymbol{\bullet}}(F ; G \mathcal{V}) \pm\left(\begin{array}{l}
f \\
2
\end{array}\right) \gamma=\mathrm{t}^{\boldsymbol{\bullet}}(F ; G / \mathcal{V}) \pm\left(\left(\begin{array}{l}
f \\
2
\end{array}\right) \gamma+\frac{2 k f}{n}\right) .
$$

Proof. Since $\mathcal{V}$ is a $\gamma$-FK-regular equipartition of $G \in \mathcal{G}$, we have $\left\|G-G_{\mathcal{V}}\right\|_{\square} \leq \gamma$. Hence, by Lemma 3.20 we get

$$
\mathrm{t}^{\bullet}(F ; G)=\mathrm{t}^{\bullet}(F ; G \mathcal{V}) \pm\left(\begin{array}{l}
f \\
2
\end{array}\right) \gamma .
$$

The other inequality follows from Proposition 3.4.

Lemma 3.23 ([BCL ${ }^{+} 08$, Theorem 2.7(a)]). Let $\mathcal{V}$ be a $\gamma$-FK-regular equipartition of a graph $G \in \mathcal{G}$. Then, for any graph $F \in \mathcal{G}$ we have

$$
\mathrm{t}(F ; G)=\mathrm{t}\left(F ; G_{\mathcal{V}}\right) \pm \mathrm{e}(F) \gamma=\mathrm{t}(F ; G / \mathcal{V}) \pm\left(\mathrm{e}(F) \gamma+\frac{2 k f}{n}\right)
$$

Proof. Since $\mathcal{V}$ is a $\gamma$-FK-regular equipartition of $G \in \mathcal{G}$, we have $\left\|G-G_{\mathcal{V}}\right\|_{\square} \leq \gamma$. Hence, by Lemma 3.21 we get

$$
\mathrm{t}(F ; G)=\mathrm{t}(F ; G \mathcal{V}) \pm \mathrm{e}(F) \gamma
$$

The other inequality follows from Proposition 3.4.

We finish this section by noting that one can mimic the proof of Lemma 3.21 for bipartite graphs to get the following result.

Lemma 3.24. Let $H \in \mathcal{H}_{[0,1]}(X, Y)$ and $H^{\prime} \in \mathcal{H}_{[0,1]}(X, Y)$ be two weighted bipartite graphs over $(X, Y)$ and let $F=([s],[t], E(F))$ be a bipartite graph. Then

$$
\left|\ddot{\mathrm{t}}_{F}(H)-\ddot{\mathrm{t}}_{F}\left(H^{\prime}\right)\right| \leq \mathrm{e}(F)\left\|H-H^{\prime}\right\|_{\square} \text {. }
$$

Although we will not use Lemma 3.24 in the remainder of this thesis, we notice that it can be used to improve the constant in the second item of Lemma 3.8.

Corollary 3.25. Let $H \in \mathcal{H}_{[0,1]}(X, Y)$ be a weighted bipartite graph. If $(X, Y)$ is $\frac{1}{4} \gamma$-regular, then $(X, Y)$ is $\gamma$-quad-regular.

Proof. First, we define $\Delta=H-d$, where $d=\mathrm{d}(X, Y)=H(X, Y) /|X||Y|$. By Proposition 3.6 we must have $\|\Delta\|_{\square} \leq \frac{1}{4} \gamma$. 
Hence, one can apply Lemma 3.24 with $H^{\prime}=d$ to get

$$
\left|\ddot{\mathrm{t}}_{C_{4}}(H)-\ddot{\mathrm{t}}_{C_{4}}(d)\right| \leq 4\|\Delta\|_{\square} \leq \gamma \text {. }
$$

Since $\ddot{\mathrm{t}}_{C_{4}}(d)=d^{4}$, we obtain $\ddot{\mathrm{t}}_{C_{4}}(H) \leq d^{4}+\gamma$, that is, $H=(X, Y)$ is $\gamma$-quad-regular.

\subsection{Removal Lemmas}

In this section and the next, we discuss a family of deep results that have become known as Removal Lemmas.

\subsubsection{Removal Lemma for a single graph}

A Removal Lemma for a graph $F$ states that graphs that are far from being $F$-free must have many copies of $F$.

Lemma 3.26 (Removal Lemma). For every $\varepsilon>0$ and every graph $F$, there exists $\delta=\delta(\varepsilon, F)>0$ for which the following holds. If $G$ is an n-vertex graph, with $n \geq \delta^{-1}$, satisfying $\mathbf{d}_{1}(G, \operatorname{Forb}(F)) \geq \varepsilon$, then $\mathrm{p}(F ; G) \geq \delta$.

In other words, Lemma 3.26 above states that graphs with at most $\delta n^{|V(F)|}$ copies of $F$ can be made $F$-free by removing at most $\varepsilon n^{2}$ edges. The Removal Lemma for triangles (i.e. for $F=K_{3}$ ) was proved by Ruzsa and Szemerédi in [RS78]. The more general version above was first stated explicitly in the literature by Alon, Duke, Lefmann, Rödl and Yuster [ADL $\left.{ }^{+} 94\right]$ and by Füredi [Für95].

Lemma 3.26 can be proved by regularity methods. Such proofs bounds $\delta^{-1}$ by $\operatorname{TOWER}\left(\operatorname{poly}\left(\varepsilon^{-1}\right)\right)$. Fox [Fox11] (cf. [MS19]) showed that Lemma 3.26 holds with $\delta^{-1}$ bounded by TOWER $\left(O\left(\log \left(\varepsilon^{-1}\right)\right)\right)$. Moreover if $F$ is bipartite, then $\delta^{-1}$ is polynomial in $\varepsilon^{-1}$ and, though it is not possible to get polynomial bounds when $F$ is not bipartite (see [Alo02]), the best known lower bound for $\delta^{-1}$ is only quasi-polynomial in $\varepsilon^{-1}$, i.e., we only know that $\delta^{-1} \geq(1 / \varepsilon)^{\text {poly }(\log (1 / \varepsilon))}$.

\subsubsection{Removal Lemma for families of graphs}

One can immediately derive a version of Lemma 3.26 for finite families $\mathcal{F}$, since a graph $\varepsilon$-far from $\operatorname{Forb}(\mathcal{F})$ must be $\varepsilon /|\mathcal{F}|$-far from $\operatorname{Forb}(F)$, for at least one member $F \in \mathcal{F}$. Alon and Shapira [AS08b] proved that the Removal Lemma holds for arbitrary (possibly infinite) families $\mathcal{F}$ of graphs, by making use of the Strong Regularity Lemma (Lemma 3.17). That result, which will be useful in Chapter 8 , is stated below.

Lemma 3.27 (Removal Lemma for $\mathcal{F}$ ). For every $\varepsilon>0$ and every (possibly infinite) family $\mathcal{F}$ of graphs, there exist $M=M(\varepsilon, \mathcal{F}), \delta=\delta(\varepsilon, \mathcal{F})>0$ and $n_{0}=n_{0}(\varepsilon, \mathcal{F})$ such that the following holds. If a graph $G$ on $n \geq n_{0}$ vertices satisfies $\mathbf{d}_{1}(G, \operatorname{Forb}(\mathcal{F})) \geq \varepsilon$, then there is $F \in \mathcal{F}$ with $|V(F)| \leq M$ such that $G$ has at least $\delta n^{|V(F)|}$ copies of $F$.

Conlon and Fox [CF12] showed that Lemma 3.27 holds with $\delta^{-1}, n_{0} \leq \operatorname{TOWER}\left(\operatorname{poly}\left(\varepsilon^{-1}\right)\right)$. Although this remains the best known bound for the general case, there are many families $\mathcal{F}$ for which Lemma 3.27 holds with significantly better dependency on $\varepsilon$. Lemma 3.27 also holds with $\delta^{-1}, M, n_{0} \leq \operatorname{poly}\left(\varepsilon^{-1}\right)$ for certain infinite families $\mathcal{F}$. For instance, results from [GGR98] 
provide such polynomial bounds when $\operatorname{Forb}(\mathcal{F})$ is the property of "being $k$-colorable" (for every fixed integer $k$ ) or the property of "having a bisection of size at most $\rho n^{2}$ " (for every fixed $\rho>0$ ) or many other properties that can be expressed as "partition problems".

\subsubsection{Induced Removal Lemma}

We recall that for graphs $F$ and $\Gamma$, we let $\mathrm{t}_{\hookrightarrow}^{\bullet}(F ; \Gamma)$ be the probability that a random mapping $\varphi: V(F) \rightarrow V(\Gamma)$ is an induced homomorphism, i.e., a function preserving adjacency and non-adjacency between $F$ and $\Gamma$. The following seminal result, proved by Alon and Shapira [AS08a], will be important in Chapter 9 .

Lemma 3.28 (Induced Removal Lemma). For every $\varepsilon>0$ and every (possibly infinite) family $\mathcal{F}$ of graphs, there exist $M=M_{3.28}(\varepsilon, \mathcal{F}), \delta=\delta_{3.28}(\varepsilon, \mathcal{F})>0$ and $n_{0}=n_{3.28}^{(0)}(\varepsilon, \mathcal{F})$ such that the following holds. If a graph $\Gamma$ on $n \geq n_{0}$ vertices satisfies $\mathbf{d}_{1}(\Gamma, \operatorname{Forb}(\mathcal{F})) \geq \varepsilon$, then there is a graph $F \in \mathcal{F}$ with $|V(F)| \leq M$ such that $\mathrm{t}_{\hookrightarrow}^{\bullet}(F ; \Gamma) \geq \delta$.

This version of the induced Removal Lemma was proved using a strong version of the Regularity Lemma and therefore had upper bounds on $1 / \delta$ and $n_{0}$ of size WOWZER(poly $\left.(1 / \varepsilon)\right)$ [AFKS00]. The best known upper bound on the induced Removal Lemma is due to Conlon and Fox [CF12], but is still of tower type. Alon and Shapira [AS06] characterized all graphs $F$, with the possible exception of $F \in\left\{P_{4}, C_{4}\right\}$, such that the induced Removal Lemma for $\mathcal{F}=\{F\}$ holds for $\delta(\varepsilon, F)=\operatorname{poly}(\varepsilon)$. The case $F=P_{4}$ was shown to satisfy this property by Alon and Fox [AF15], while Gishboliner and Shapira [GS19] made progress in the case $F=C_{4}$ obtaining an single exponential bound. The question of deciding which hereditary properties admit a Removal Lemma that may be proven without the use of the Regularity Lemma was raised by Goldreich [Gol11b], and by Alon and Fox [AF15], among others, and is currently under research. 


\section{Chapter 4}

\section{Property testing and parameter estimation}

\subsection{Property testing}

Given a graph $G \in \mathcal{G}(V)$ and a graph property $\mathcal{P}$, we define the distance from $G$ to $\mathcal{P}$ as

$$
\mathbf{d}_{1}(G, \mathcal{P})=\min \left\{\mathbf{d}_{1}(G, H): H \in \mathcal{P} \text { with } V(H)=V(G)\right\}
$$

We say that $G$ is $\varepsilon$-close to $\mathcal{P}$ if $\mathbf{d}_{1}(G, \mathcal{P}) \leq \varepsilon$. Otherwise, we say that $G$ is $\varepsilon$-far from $\mathcal{P}$.

We say an algorithm has oracle access to a graph $G$ if, for every pair $u v \in V^{2}$, the algorithm can make a query to test whether $G(u, v)=0$ or $G(u, v)=1$. This is a necessary formalism required for describing a sublinear algorithm, since it should not access the entire input.

Definition 4.1 ( $\varepsilon$-tester). Given $\varepsilon>0$ and a graph property $\mathcal{P}$, an $\varepsilon$-tester $\mathcal{T}$ for $\mathcal{P}$ is a randomized decision algorithm that, when given oracle access to the input graph $G$, satisfies the following:

(i) if $G \in \mathcal{P}$, then $\mathcal{T}$ accepts $G$ with probability at least $\frac{2}{3}$;

(ii) if $G$ is $\varepsilon$-far from $\mathcal{P}$, then $\mathcal{T}$ rejects $G$ with probability at least $\frac{2}{3}$.

Moreover, if $\mathcal{T}$ accepts every graph $G \in \mathcal{P}$ with probability 1 , then $\mathcal{T}$ is said to have one-sided error.

The following simple proposition asserts that one could replace $\frac{2}{3}$ in Definition 4.1 by any constant strictly greater than $\frac{1}{2}$.

Proposition 4.2. Let $\varepsilon>0$ be fixed and suppose there is an $\varepsilon$-tester $\mathcal{T}$ for $\mathcal{P}$ which makes at most $q(\varepsilon)$ queries to the input graph. Then, for every $\delta>0$, there is an $\varepsilon$-tester $\mathcal{T}^{\prime}$ with error probability at most $\delta$, i.e., an algorithm satisfying the following:

(i) if $G \in \mathcal{P}$, then $\mathcal{T}^{\prime}$ accepts $G$ with probability at least $1-\delta$;

(ii) if $G$ is $\varepsilon$-far from $\mathcal{P}$, then $\mathcal{T}$ rejects $G$ with probability at least $1-\delta$.

Moreover, $\mathcal{T}^{\prime}$ makes at most $q^{\prime}(\varepsilon)=O(q(\varepsilon) \log (1 / \delta))$ queries to the input graph. 
Proof. We will show it is enough to consider an algorithm $\mathcal{T}^{\prime}$ that, when given as input a graph $G$, invokes $\mathcal{T}(G)$ successively $k=18\lceil\ln (2 / \delta)\rceil$ times, and then accepts $G$ if and only if $\mathcal{T}$ accepted $G$ at least $\frac{1}{2} k$ times.

Indeed, for every $i \in[k]$, let $X_{i}$ be the indicator random variable for the event that $G$ is accepted in the $i$-th run of $\mathcal{T}$ and let $S_{k}=\sum_{i=1}^{k} X_{i}$. If $G \in \mathcal{P}$, then $\mathbb{E}\left(S_{k}\right)=\sum_{i=1}^{k} \mathbb{E}\left(X_{i}\right) \geq\left(\frac{1}{2}+\frac{1}{6}\right) k$. It follows that

$$
\mathbb{P}(\mathcal{T} \text { rejects } G)=\mathbb{P}\left(S_{k}<\frac{1}{2} k\right) \leq \mathbb{P}\left(S_{k}<\mathbb{E}\left(S_{k}\right)-\frac{1}{6} k\right) .
$$

On the other hand, if $G$ is $\varepsilon$-far from $\mathcal{P}$, then $\mathbb{E}\left(S_{k}\right)=\sum_{i=1}^{k} \mathbb{E} X_{i} \leq\left(\frac{1}{2}-\frac{1}{6}\right) k$. It follows that

$$
\mathbb{P}(\mathcal{T} \text { accepts } G)=\mathbb{P}\left(S_{k} \geq \frac{1}{2} k\right) \leq \mathbb{P}\left(S_{k} \geq \mathbb{E}\left(S_{k}\right)+\frac{1}{6} k\right) .
$$

In both cases, Lemma 2.3 implies $\mathcal{T}$ outputs the "wrong" decision with probability at most

$$
2 \exp \left\{-\frac{2 k^{2}}{36 k}\right\}=2 e^{\ln \delta / 2}=\delta,
$$

as desired.

Remark 4.3. An analogous argument to the one above allows one to start with an $\varepsilon$-tester with error probability at most $\frac{1}{2}-\delta$ for any fixed $\delta>0$ (i.e., a tester which is not too reliable) and produce an $\varepsilon$-tester with error probability, say, $\frac{1}{3}$ as in Definition 4.1 (see also Proposition 10.2).

Definition 4.4 (testable, query complexity). A graph property $\mathcal{P}$ is said to be testable with query complexity $q:(0,1] \rightarrow \mathbb{N}$ if, for every $0<\varepsilon \leq 1$, there is an $\varepsilon$-tester $\mathcal{T}$ for $\mathcal{P}$ that makes at most $q(\varepsilon)$ queries to the input graph.

Remark 4.5 (uniform testing). Let $\mathcal{P}$ be a testable graph property. A uniform tester for $\mathcal{P}$ is an algorithm $\mathcal{U}$ which, when given as input $\varepsilon>0$ and oracle access to a graph $G$, behaves as an $\varepsilon$-tester for $\mathcal{P}$ with input $G$. We first remark that Definition 4.4 does not require the existence of such a uniform tester $\mathcal{U}$ for $\mathcal{P}$. We note that given any (possibly non-recursive) function $Q(\varepsilon)$, there are monotone testable properties $\mathcal{P}$ for which, for every $\varepsilon>0$, any $\varepsilon$-tester for $\mathcal{P}$ is required to make at least $Q(\varepsilon)$ queries to the input graph (see [AS08b, Theorem 4]). We refer the reader to [AS08c] for further discussions regarding the existence of uniform testers.

Remark 4.6 (query complexity independent of $|V(G)|)$ ). In the literature, sometimes a property is said to be testable if there is an $\varepsilon$-tester that makes at most $q(\varepsilon, n)$ queries, where $n=|V(G)|$ is the size of the input graph and $q(\varepsilon, n)$ is sublinear in $n$. In this thesis, we adopt the terminology in Definition 4.4.

Definition 4.7 (canonical tester, $\mathrm{ACC}_{n}$ ). An $\varepsilon$-tester $\mathcal{T}$ for a property $\mathcal{P}$ is said to be canonical if there exist an integer $q$ and a family of graph properties $\left\{\mathrm{ACC}_{n}\right\}_{n=1}^{\infty}$ for which the tester behaves as follows. When given oracle access to an input graph $G=(V, E)$ with $n:=|V| \geq q$, the tester $\mathcal{T}$ chooses a set $Q \in_{\mathrm{U}}\left(\begin{array}{l}V \\ q\end{array}\right)$ of $q$ vertices from $V$ uniformly at random and makes $\left(\begin{array}{l}q \\ 2\end{array}\right)$ queries in order to determine the induced graph $G[Q]$. Finally, the tester accepts $G$ if and only if $G[Q] \in \mathrm{ACC}_{n}$. The integer $q$ is called the sample size of $\mathcal{T}$. 
The following crucial result of Goldreich and Trevisan [GT03, Theorem 2] (see also [GT05]) allows us to assume any tester is canonical.

Theorem 4.8 ([GT03, Theorem 2]). Let $\mathcal{T}^{\prime}$ be an $\varepsilon$-tester for a graph property $\mathcal{P}$ that makes at most $q^{\prime}$ queries when given oracle access to an input graph. Then, there exists a canonical $\varepsilon$-tester $\mathcal{T}$ for $\mathcal{P}$ with sample size at most $q=O\left(q^{\prime 2}\right)$.

Definition 4.9 (testable, sample complexity). We say that a graph property $\mathcal{P}$ is testable with sample complexity $q:(0,1] \rightarrow \mathbb{N}$ if for every $\varepsilon>0$ there is a canonical tester with sample size at most $q(\varepsilon)$. In this case we say that "sample complexity of $\mathcal{P}$ " is at most $q(\varepsilon)$.

The following corollary summarizes the fact we can use the terms testable with sample complexity or testable with query complexity interchangeably, because we are not concerned with a polynomial increase in the query complexity.

Corollary 4.10. Let $\mathcal{P}$ be a graph property.

(i) If $\mathcal{P}$ is testable with query complexity $q^{\prime}(\varepsilon)$, then $\mathcal{P}$ is testable with sample complexity $q(\varepsilon)$, with $q(\varepsilon)=O\left(q(\varepsilon)^{2}\right)$.

(ii) If $\mathcal{P}$ is testable with sample complexity $q(\varepsilon)$, then $\mathcal{P}$ is testable with query complexity $q^{\prime}(\varepsilon)$, with $q^{\prime}(\varepsilon)=\left(\begin{array}{c}q(\varepsilon) \\ 2\end{array}\right)=O\left(q(\varepsilon)^{2}\right)$.

Definition 4.11 (easily testable). A property is $\mathcal{P}$ is said to be easily testable if, for every $\varepsilon>0$, there is an $\varepsilon$-tester for $\mathcal{P}$ with sample complexity $q(\varepsilon)=\operatorname{poly}\left(\varepsilon^{-1}\right)$.

\subsubsection{Testability of hereditary properties and Induced Removal Lemmas}

Note that the Induced Removal Lemma (Lemma 3.28) implies that every hereditary graph property $\mathcal{P}=\operatorname{Forb}^{\bullet}(\mathcal{F})$ can be tested by sampling a set $Q \in_{\mathrm{U}}\left(\begin{array}{l}V \\ q\end{array}\right)$, with $q=\operatorname{poly}\left(\delta_{3.28}(\varepsilon)^{-1}, M_{3.28}(\varepsilon)\right)$, from the input graph $G=(V, E)$ and accepting if and only if $G[Q] \in \mathcal{P}$.

Conversely, a moment's thought reveals that the proposition below has the following consequence: the testability of $\mathcal{P}=\operatorname{Forb}^{\boldsymbol{O}}(\mathcal{F})$ in fact implies the existence of an Induced Removal Lemma for $\mathcal{F}$.

Proposition 4.12. Let $\mathcal{P}$ be a hereditary graph property and suppose $\mathcal{P}$ is testable with query complexity $q(\varepsilon)$. Then, for every $\varepsilon>0$, there is $q^{\prime}=\operatorname{poly}(q(\varepsilon))$ such that the algorithm that samples a set $Q \in_{U}\left(\begin{array}{l}V \\ q^{\prime}\end{array}\right)$ of the input graph $G=(V, E)$ and accepts if and only if $G[Q] \in \mathcal{P}$ is an $\varepsilon$-tester for $\mathcal{P}$ with one-sided error.

Proof. See [GT03, Proposition D.2]. For an analogous argument for configuration of points see the proof of Theorem 11.3.

Inspecting the discussion above connecting the testability of $\mathcal{P}=\operatorname{Forb}(\mathcal{F})$ and the Induced Removal Lemma for $\mathcal{F}$, one sees that the numerical parameters involved are polynomially related. Let $\delta=\delta_{3.28}(\varepsilon, \mathcal{F})$ and $M=M_{3.28}(\varepsilon, \mathcal{F})$ be the parameters in the Induced Removal Lemma (Lemma 3.28), and let $q$ be the sample complexity required to test $\mathcal{P}=\operatorname{Forb}(\mathcal{F})$. We then have $\delta^{-1}=\operatorname{poly}(q), M=\operatorname{poly}(q)$ and $q=\operatorname{poly}\left(\delta^{-1}, M\right)$. 


\subsection{Parameter estimation}

A function $z: \mathcal{G} \rightarrow \mathbb{R}$ from the set $\mathcal{G}$ of all finite graphs into the real numbers is called a graph parameter if it is invariant under isomorphism. We also define weighted graph parameters as functions that are invariant under weight-preserving relabeling.

The following function, which will be studied in this thesis, is a typical example of a graph parameter.

Definition $4.13\left(\mathbf{d}_{\mathcal{P}}\right)$. For every graph property $\mathcal{P}$, define the graph parameter

$$
\begin{aligned}
\mathbf{d}_{\mathcal{P}}: \mathcal{G} & \rightarrow[0,1] \\
\Gamma & \mapsto \mathbf{d}_{1}(\Gamma, \mathcal{P})
\end{aligned}
$$

that maps every graph $\Gamma$ to its distance from $\mathcal{P}$.

A graph parameter $f: \mathcal{G} \rightarrow \mathbb{R}$ is "estimable" if it can be be approximated up to an additive error by just inspecting a very small sample of the input graph.

Definition 4.14 (estimable). A graph parameter $f: \mathcal{G} \rightarrow \mathbb{R}$ is estimable if there exists a function $q:(0,1] \rightarrow \mathbb{N}$ such that, for every $\varepsilon>0$, and every graph $\Gamma=(V, E)$,

$$
\mathbb{P}(|f(\Gamma)-f(\Gamma[Q])| \leq \varepsilon) \geq \frac{2}{3},
$$

where $Q \in_{\mathrm{U}}\left(\begin{array}{l}\Gamma \\ q\end{array}\right)$. We say that $f$ is estimable with sample complexity $q$ for every $q$ as above..

\subsection{Literature}

In this short section, we discuss literature directly relevant to this thesis, from a more general point of view.

For more information about property testing we refer the reader to [Gol11a] and the references therein.

\subsubsection{Property testing}

The systematic study of property testing was initiated by Goldreich, Goldwasser and Ron [GGR98], who established the testability of many graph properties, such as $k$-colorability. Another seminal paper is [GT03], in which Goldreich and Trevisan showed (among other results) that property testing can be reduced to making decisions based on the subgraph induced by a sample of vertices chosen uniformly at random from the input graph (see Theorem 4.8).

The fact that $\operatorname{Forb}(F)$ is testable was implicitly known, because it is a consequence of the Removal Lemma for $F$ (see Section 3.6). The induced removal lemma for a single graph $F$ was later established in [AFKS00], and as a consequence the testability of $\operatorname{Forb}^{\bullet}(F)$ was proved. Extensions of such removal lemmas for infinite families led to the following more general results: Alon and Shapira [AS08b] proved that every monotone graph property is testable, and, more generally, that the same holds for hereditary graph properties [AS08a]. We remark that, conversely, the testability of any given hereditary property $\operatorname{Forb}^{\bullet}(\mathcal{F})$ also implies (see Proposition 4.12) the existence of 
an induced Removal Lemma for $\mathcal{F}$ (with numerical constants polynomially related to the sample complexity of the tester).

Finally, Alon, Fischer, Newman and Shapira [AFNS09] found a combinatorial characterization of testable graph properties based on regular partitions. In contrast, an analytic characterization of testable graph properties was obtained in $\left[\mathrm{BCL}^{+} 08\right]$.

\subsubsection{Efficient property testing}

Once a property is shown to be testable, it becomes relevant to determine the sample complexity required to test it.

Quite remarkably, Goldreich, Goldwasser and Ron, already in [GGR98], established a rich family of properties to be easily testable. Notably, this family includes $k$-colorability, for which testability was previously known (implicitly) only with tower-type sample complexity [RD85].

Let us now turn to properties of type $\operatorname{Forb}(F)$. Alon [Alo02] proved that $\operatorname{Forb}(F)$ is easily testable with one-sided error if and only if $F$ is bipartite. By Proposition 4.12, it actually follows that $\operatorname{Forb}(F)$ is easily testable if and only if $F$ is bipartite. We remark in passing that, also by the discussion in Section 4.1.1, the sample complexity of testing $\operatorname{Forb}(F)$ is tied to the numerical constants of the Removal Lemma for $F$ (see Section 3.6). In particular, the best known general upper bound for the sample complexity of testing $\operatorname{Forb}(F)$ is $\operatorname{TOWER}(\log (1 / \varepsilon))$, due to Fox [Fox11].

The property $\operatorname{Forb}^{\bullet}(F)$ is known to be easily-testable for $F \in\left\{P_{2}, \overline{P_{2}}, P_{3}, \overline{P_{3}}\right\}$ [AS06] and for $F \in\left\{P_{4}, \overline{P_{4}}\right\}$ [AF15]. For every other graph $F \notin\left\{C_{4}, \overline{C_{4}}\right\}$, we know that $\operatorname{Forb}^{\bullet}(F)$ is not easily-testable [AS06]. Recently, Gishboliner and Shapira [GS19] proved that Forb $^{\bullet}\left(C_{4}\right)$ (and, symmetrically, $\left.\operatorname{Forb}^{\bullet}\left(\overline{C_{4}}\right)\right)$ can be tested with sample complexity $\operatorname{EXP}(\operatorname{poly}(1 / \varepsilon))$. They also showed chordality can be tested with sample complexity $\operatorname{EXP}(\operatorname{poly}(1 / \varepsilon))$, which was later improved to poly $(1 / \varepsilon)$ by De Joannis de Verclos [dJdV19]. In general, the best known upper bound for the sample complexity of testing $\operatorname{Forb}^{\bullet}(F)$ is due to Conlon and Fox [CF12], but it is still of tower type.

Gishboliner and Shapira [GS17] obtained positive and negative results for families $\mathcal{F}$ to be such that $\operatorname{Forb}^{\bullet}(\mathcal{F})$ is easily testable. In particular, they showed that if a finite family $\mathcal{F}$ contains a bipartite graph, a split-graph and a cobipartite graph, then $\operatorname{Forb}^{\bullet}(\mathcal{F})$ is easily testable. In the other direction, they showed that every finite family $\mathcal{F}$ for which $\operatorname{Forb}^{\bullet}(\mathcal{F})$ is easily testable must contain a bipartite and a cobipartite graph. Despite the efforts of several authors, a full characterization of families $\mathcal{F}$ for which $\operatorname{Forb}^{\bullet}(\mathcal{F})$ is easily testable is still missing.

\subsubsection{Parameter estimation}

Estimable parameters were considered in [FN07] and are often also called testable parameters in the literature.

Most of the literature regarding estimable graph parameters focus on the parameter $\mathbf{d}_{\mathcal{P}}$, which gives the distance to a given property $\mathcal{P}$. Estimating $\mathbf{d}_{\mathcal{P}}$ is closely related to the notion of tolerant testing, which was introduced by Parnas, Ron and Rubinfeld [PRR06], and is a generalization of standard property testing. Let $0 \leq \rho_{1}<\rho_{2} \leq 1$. A $\left(\rho_{1}, \rho_{2}\right)$-tolerant tester for a graph property $\mathcal{P}$ is an algorithm that receives a graph $\Gamma$ as input and distinguishes between the cases $\mathbf{d}_{1}(\Gamma, \mathcal{P}) \leq \rho_{1}$ and $\mathbf{d}_{1}(\Gamma, \mathcal{P}) \geq \rho_{2}$ with probability at least $2 / 3$ and constant query complexity.

In a seminal piece of work, Fischer and Newman [FN07] proved that every testable graph property $\mathcal{P}$ has a $(d-\varepsilon, d)$-tolerant tester, for every positive $d$ and $\varepsilon$. The distance from a graph to 
$\mathcal{P}$ can then be estimated by successively running such tolerant testers. In other words, they showed that a graph property $\mathcal{P}$ is testable if, and only if, $\mathbf{d}_{\mathcal{P}}$ is estimable.

Working in a different framework, Borgs, Chayes, Lovász, Sós and Vesztergombi $\left[\mathrm{BCL}^{+} 08\right.$, Theorem 6.1] gave a complete characterization of the graph parameters that are estimable. In particular, their result implies that the distance $\mathbf{d}_{\mathcal{P}}$ from a hereditary graph property $\mathcal{P}$ is estimable. Their work uses the concept of graph limits and does not give explicit bounds on the query complexity required for this estimation.

\subsubsection{Efficient parameter estimation}

Given a testable property $\mathcal{P}$, Fischer and Newman [FN07] gave explicit upper bounds for the sample complexity of estimating $\mathbf{d}_{\mathcal{P}}$. However, such bounds are much larger than the sample complexity $q(\varepsilon)$ of testing $\mathcal{P}$ - more specifically, the sample complexity obtained in [FN07] is at least $\operatorname{TOWER}(q(\varepsilon))$.

For monotone properties $\mathcal{P}=\operatorname{Forb}(\mathcal{F})$, Alon, Shapira and Sudakov [ASS09] proved an upper bound for the sample complexity of estimating $\mathbf{d}_{\mathcal{P}}$ which depends only on the parameters given by Lemma 3.18. In particular, for finite families $\mathcal{F}$, their result gives a sample complexity of $\operatorname{TOWER}\left(\operatorname{poly}\left(\varepsilon^{-1}\right)\right)$ for estimating $\mathbf{d}_{\mathcal{P}}$. We remark that their approach can be extended to hereditary properties $\mathcal{P}=\operatorname{Forb}^{\bullet}(\mathcal{F})$.

It follows that the bounds given by [ASS09] for the sample complexity of estimating $\mathbf{d}_{\mathcal{P}}$ essentially match the ones for the sample complexity of testing $\mathcal{P}$, for typical, "hard" monotone (and also hereditary) properties $\mathcal{P}$. However, any approach relying on the regularity lemma (as in the case of [ASS09]) will always leave a huge gap between the sample complexity of testing $\mathcal{P}$ and of estimating $\mathbf{d}_{\mathcal{P}}$ for properties $\mathcal{P}$ that are efficiently testable (such as easily testable ones or even properties such as $\operatorname{Forb}^{\bullet}\left(C_{4}\right)$ that are testable with exponential sample complexity).

In $\left[\mathrm{HKL}^{+} 16, \mathrm{HKL}^{+} 20, \mathrm{HKL}^{+} 17, \mathrm{HKL}^{+}\right.$] (see also Chapters 8 and 9), upper bounds are given for the sample complexity of estimating the distance $\mathbf{d}_{\mathcal{P}}$ from a hereditary graph property $\mathcal{P}=\operatorname{Forb}^{\bullet}(\mathcal{F})$. Those bounds depend only on the numerical parameters of the induced removal lemma for $\mathcal{F}$. In particular, the aforementioned gap is greatly reduced for families $\mathcal{F}$ for which there is an efficient removal lemma, that is, for properties $\mathcal{P}=\operatorname{Forb}^{\bullet}(\mathcal{P})$ which can be efficiently tested.

Very recently, Fiat and Ron [FR20] provided a framework that, in some sense, relates to the ones described in Chapters 8 and 9. They showed that $\operatorname{Forb}^{\bullet}\left(P_{3}\right), \operatorname{Forb}^{\boldsymbol{\Theta}}\left(P_{4}\right)$ and chordality are properties $\mathcal{P}$ for which $\mathbf{d}_{\mathcal{P}}$ can be estimated with polynomial sample complexity. They also proved that distance to $\operatorname{Forb}^{\bullet}\left(C_{4}\right)$ can be estimated with exponential sample complexity. 


\section{Chapter 5}

\section{Partitions of a graph and sampling}

In order to motivate the results in this chapter, let us consider the property $\mathcal{P}$ of graphs $G$ having an equipartition $\left\{V_{1}, V_{2}\right\}$ of density at least $\frac{1}{4}$. This property is known to be testable (see [GGR98]; this will also be a consequence of Theorem 5.1 below). Therefore, it must admit a canonical $\varepsilon$-tester, i.e., a tester that accepts the input graph $G=(V, E)$ if and only if $G[Q] \in \operatorname{ACC}_{n}$, where $Q \in_{\mathrm{U}}\left(\begin{array}{l}V \\ q\end{array}\right)$, $\mathrm{ACC}_{n}$ is some graph property and $n=|V|$. A very natural candidate for $\mathrm{ACC}_{n}$ is the property of graphs admitting an equipartition $\left\{Q_{1}, Q_{2}\right\}$ with $\mathrm{d}\left(Q_{1}, Q_{2}\right) \geq \frac{1}{4}-\varepsilon^{\prime}$, for some small $\varepsilon^{\prime}>0$.

Indeed, if $G \in \mathcal{P}$, that is, if $G$ has an equipartition $\left\{V_{1}, V_{2}\right\}$ with $\mathrm{d}\left(V_{1}, V_{2}\right) \geq 1 / 4$, then set $Q_{i}^{\prime}:=V_{i} \cap Q$ for $i=1,2$. By standard concentration results (see Lemma 2.5), one can show that, with high probability, $\left|Q_{i}^{\prime}\right|=\left(\frac{1}{2} \pm \delta\right) q(i=1,2)$ and $\mathrm{e}\left(Q_{1}^{\prime}, Q_{2}^{\prime}\right) \geq \frac{1}{4}(q / 2)^{2} \pm \delta q$, for some $\delta=O(\varepsilon)$. By arbitrarily moving up to $\delta q$ vertices from one part to another, one ends up with an equipartition $\left\{Q_{1}, Q_{2}\right\}$ of $G[Q]$ with density at least $\mathrm{d}\left(Q_{1}, Q_{2}\right) \geq \frac{1}{4}-\varepsilon^{\prime}$, for a suitable $\varepsilon^{\prime}>0$.

Unfortunately, proving that the tester behaves correctly when $G$ is $\varepsilon$-far from $\mathcal{P}$ is not that straightforward. In that case, we know that there can be no equipartition $\left\{V_{1}, V_{2}\right\}$ of $G$ with $\mathrm{d}\left(V_{1}, V_{2}\right)>\frac{1}{4}-\varepsilon$. But it is not clear whether this fact implies, with high probability, that there is no equipartition $\left\{Q_{1}, Q_{2}\right\}$ of $G[Q]$ with $\mathrm{d}\left(Q_{1}, Q_{2}\right)>\frac{1}{4}-\varepsilon^{\prime}$.

In this chapter, we show that the above implication does hold, by proving the following more general result about equipartitions. For any given $\varepsilon>0$ and integer $k$, we show that if a typical sample $G[Q]$ of size $q=|Q|=\operatorname{poly}\left(\varepsilon^{-1}, k\right)$ has an equipartition into $k$ classes with densities $\left\{d_{i j}\right\}_{i, j=1}^{k}$, then $G$ has an equipartition into $k$-classes with densities $\left\{d_{i j} \pm \varepsilon\right\}_{i, j=1}^{k}$.

Theorem 5.1. For every $\varepsilon>0, \delta>0$, and every integer $k>0$, there is an integer $q_{5.1}=$ $q_{5.1}(\varepsilon, k, \delta)=\operatorname{poly}\left(\varepsilon, k, \log \frac{1}{\delta}\right)>0$ satisfying the following. Let $Q$ be a set of $q \geq q_{5.1}$ vertices taken uniformly at random from a graph $G$ of size $n=|V(G)| \geq q$. Then, with probability at least $1-\delta$, the following holds.

(i) For every equipartition $\left\{Q_{i}\right\}_{i=1}^{k}$ of $G[Q]$, there is an equipartition $\left\{V_{i}\right\}_{i=1}^{k}$ of $G$ satisfying

$$
\mathrm{d}\left(V_{i}, V_{j}\right)=\mathrm{d}\left(Q_{i}, Q_{j}\right) \pm \varepsilon
$$

for every $1 \leq i \leq j \leq k$.

(ii) For every equipartition $\left\{V_{i}\right\}_{i=1}^{k}$ of $G$, there is an equipartition $\left\{Q_{i}\right\}_{i=1}^{k}$ of $G[Q]$ satisfying

$$
\mathrm{d}\left(Q_{i}, Q_{j}\right)=\mathrm{d}\left(V_{i}, V_{j}\right) \pm \varepsilon
$$


for every $1 \leq i \leq j \leq k$.

The next main result of this chapter is an extended version of Theorem 5.1, which assert that the equipartitions of a graph and the equipartitions of a typical sample are related, not only in terms of the densities between pair of classes, but also in terms of how regular each pair of classes are. For convenience, we state our result using the notion of quad-regular pair (Section 3.2), which is closely related to the more usual notion on regularity (see Lemma 3.8).

Theorem 5.2. For every $\varepsilon>0, \delta>0$ and every integer $k>0$, there is an integer $q_{5.2}=$ $q_{5.2}(\varepsilon, k, \delta)=\operatorname{poly}\left(\varepsilon^{-1}, k, \log \delta^{-1}\right)>0$ satisfying the following. Let $Q$ be a set of $q \geq q_{5.2}$ vertices taken uniformly at random from a graph $G$ of size $n=|V(G)| \geq q e^{\mathrm{poly}\left(\varepsilon^{-1}\right)}$.

Then, with probability at least $1-\delta$, the following hold alongside with assertions (i) and (ii) from Theorem 5.1 .

(iii) For every equipartition $\left\{Q_{i}\right\}_{i=1}^{k}$ of $G[Q]$, there is an equipartition $\left\{V_{i}\right\}_{i=1}^{k}$ of $G$ satisfying

$$
\operatorname{Reg}_{\mathfrak{a}_{0}}\left(V_{i}, V_{j}\right) \leq \operatorname{Reg}_{\mathfrak{a x o}_{0}}\left(Q_{i}, Q_{j}\right)+\varepsilon
$$

for every $1 \leq i \leq j \leq k$.

(iv) For every equipartition $\left\{V_{i}\right\}_{i=1}^{k}$ of $G$, there is an equipartition $\left\{Q_{i}\right\}_{i=1}^{k}$ of $Q$ satisfying

$$
\operatorname{Reg}_{\mathscr{z}_{0}}\left(Q_{i}, Q_{j}\right) \leq \operatorname{Reg}_{\mathscr{a}_{0}^{0}}\left(V_{i}, V_{j}\right)+\varepsilon
$$

for every $1 \leq i \leq j \leq k$.

A result similar to Theorem 5.2 - stating that a graph and a typical sample have similar regular partitions - was proven in [AFNS09, Lemma 5.2]. Since their proof relies on first applying the regularity lemma in $G$, a similar proof would give bounds for $q$ that depends on the size of a $\gamma^{\prime}$-regular-partition. Moreover, the bound for $q_{5.2}$ has no dependency on the actual values of $\operatorname{Reg}_{g_{0}}\left(V_{i}, V_{j}\right)$ (or $\left.\operatorname{Reg}_{g_{\infty}}\left(Q_{i}, Q_{j}\right)\right)$ being considered.

Next we give a brief outline of the proof of Theorem 5.1 and Theorem 5.2. In Section 5.2, we show that given a family $\left\{S_{r}\right\}_{r=1}^{t}$ of subsets of a ground set $V$, and a typical sample $Q$ of $V$, for each equipartition of $Q$ (respectively $V$ ), there is an equipartition of $V$ (respectively $Q$ ) that has a similar "intersection profile" on $\left\{S_{r}\right\}_{r=1}^{t}$, i.e., that intersects each $S_{r}$ in a similar way. We then proceed to prove Theorem 5.1 in Section 5.3 by showing that, for every graph $G$, there is a certain family $\left\{S_{r}\right\}_{r=1}^{t}$ - which will be essentially given by its cut decomposition (Lemma 3.14) — which allows one to approximately compute the densities $\mathrm{d}\left(V_{i}, V_{j}\right)$ of a partition $\left\{V_{i}\right\}_{i=1}^{t}$ based solely on the "intersection profile" $\left\{V_{i} \cap S_{r}\right\}_{r}^{i}$ of $\left\{V_{i}\right\}_{i=1}^{k}$ on $\left\{S_{r}\right\}_{r=1}^{t}$. Finally, we prove Theorem 5.2 by considering a richer family $\left\{S_{r}\right\}_{r=1}^{t}$, for which the "intersection profile" of a partition $\left\{V_{i}\right\}_{i=1}^{t}$ on it recovers not only the densities $\mathrm{d}\left(V_{i}, V_{j}\right)$ but also the homomorphism densities $\ddot{\mathrm{t}}_{C_{4}}\left(V_{i}, V_{j}\right)$.

\section{$5.1 \quad$ Related work}

There is much literature related with Theorem 5.1, primarily for the case $k=2$. Frieze and Kannan [FK99] proved that one can approximate the MAxCUT of a graph $G$ (up to an additive error 
or $\varepsilon n^{2}$ ) by sampling only a constant $f(\varepsilon)$ number of vertices from $G$. A number of other works make use of sampling to approximate the MAXCUT of a graph (see for instance [dlV96, AKK99, MS08]).

Alon, de la Vega, Kannan and Karpinski [AFdlVKK03] showed, in particular, that one can approximate the MAXCUT of a graph (up to an additive error of $\varepsilon n^{2}$ ) directly by the MAXCUT of a typical sample of size poly $\left(\varepsilon^{-1}\right)$ - this differs from the aforementioned results, which relied in more convoluted (randomized) computations.

For arbitrary values of $k$, Goldreich, Goldwasser and Ron [GGR98] provided a way of testing (in the sense of property testing) if a graph has an equipartition with a given set of densities $\left\{d_{i j}\right\}_{i, j=1}^{k}$. We study a generalization of this problem in Chapter 6. From the result in [GGR98], one can actually recover the main statement of Theorem 5.1. However, such a proof of Theorem 5.1 would not only be more complex, but would lead to a bound for $q$ which is exponential in $k$ - the tester given in [GGR98] has sample complexity poly $\left(\varepsilon 2^{k}\right)$.

Summing up, the main contribution of Theorem 5.1 when compared to previous results in the literature is a bound which is polynomial in both $\varepsilon$ and $k$.

An easy corollary of Theorem 5.1 is that, given any family $\mathcal{S} \subseteq \mathcal{G}^{*}([k])$ of weighted graphs, one can estimate, within an additive error of $\varepsilon$, the distance

$$
\mathrm{d}_{\mathcal{S}}^{*}(G)=\min \left\{\mathbf{d}_{1}(G / \mathcal{V}, \mathcal{S}): \text { for every } \mathcal{V} \in \wp_{k}(G)\right\}
$$

with sample complexity poly $(1 / \varepsilon, k)$. Indeed, one can simply output $\mathrm{d}_{\mathcal{S}}^{*}(G[Q])$, where $Q$ is a sample of $q=q_{5.1}(\varepsilon, k)$ vertices, and the result follows from the two assertions in Theorem 5.1 together. Very recently, Fiat and Ron [FR20, Lemma 1.1] showed that one can estimate the distance $\mathrm{d}_{\mathcal{S}}^{*}(G)$ with sample complexity poly $(1 / \varepsilon, \log k)$ if $\mathcal{S}$ is a "semi-homogeneous" family of weighted graphs. We call a family $\mathcal{S} \subseteq \mathcal{G}^{*}([k])$ of weighted graphs semi-homogeneous if there are graphs $R_{0}, R_{1} \in \mathcal{G}([k])$, with $R_{1} \leq R_{0}$, for which, $\mathcal{S}=\mathcal{S H}\left(R_{0}, R_{1}\right)=\left\{R \in \mathcal{G}^{*}([k]): R_{1} \leq R \leq R_{0}\right\}$. Note that $R \in \mathcal{S H}\left(R_{0}, R_{1}\right)$ if and only if $R(i, j)=0$ for every ij $\notin E\left(R_{0}\right)$ (i.e., the non-edges of $R_{0}$ force 0 -valued entries in $R$ ) and $R(i, j)=1$ for every $i j \in E\left(R_{1}\right)$ (i.e., the edges of $R_{1}$ force 1-valued entries in $R$ ). Every other pair $i j$ is not constrained. The better bound on the estimation of $\mathrm{d}_{\mathcal{S}}^{*}(G)$, when $\mathcal{S}$ is semi-homogeneous, is then used by Fiat and Ron to provide upper bounds for estimating the distance to certain hereditary graphs properties. Their approach is similar to the one we present here in Chapters 8 and 9 and we further discuss their results at the end of Section 9.1.

\subsection{Intersection profile with a family of given sets}

Let $V$ be a ground set of size $n$ and let $\left\{S_{r}\right\}_{r=1}^{t}$ be a family of subsets of $V$. For any equipartition $\left\{U_{i}\right\}_{i=1}^{k}$ of a subset $U \subseteq V$, we refer to the following (normalized) intersection sizes $\left\{\mid U_{i} \cap\right.$ $\left.S_{r}|/| U \mid\right\}_{r \in[t]}^{i \in[k]}$ as the intersection profile of $\left\{U_{i}\right\}_{i=1}^{k}$ on $\left\{S_{r}\right\}_{r=1}^{t}$.

In this section, we will show the following key Lemma 5.3, whose proof was inspired in the proof of [AFdlVKK03, Theorem 9] (regarding random sub-programs of a linear program). Roughly speaking, Lemma 5.3 asserts that if $V$ has no partition with an intersection profile on $\left\{S_{r}\right\}_{r=1}^{t}$ close to some given values $\left\{\alpha_{r}^{(i)}\right\}$, then a typical sample $Q \subseteq V$ has no such partition as well.

Lemma 5.3. Let $t$ and $k$ be positive integers and $\eta>0$. Let $\left\{S_{r}\right\}_{r=1}^{t}$ be sets of vertices of a ground set $V$ of size $|V|=n \geq t k / \eta$. Suppose there are numbers $\left\{\alpha_{r}^{(i)}\right\}_{i \in[k], r \in[t]}$ in $[0,1]$ for which no 
partition $\left\{V_{i}\right\}_{i=1}^{k}$ of $V$ satisfies

$$
\forall r \in[t], \forall i \in[k]: \frac{\left|S_{r} \cap V_{i}\right|}{n}=\alpha_{r}^{(i)} \pm 3 \eta
$$

Then, with probability at least $1-e^{q \eta^{2} / 2}$, a set $Q \in_{U}\left(\begin{array}{c}V \\ q\end{array}\right)$ of $q$ elements chosen uniformly at random has no partition $\left\{Q_{i}\right\}_{i=1}^{k}$ satisfying

$$
\forall r \in[t], \forall i \in[k]: \frac{\left|S_{r} \cap Q_{i}\right|}{q}=\alpha_{r}^{(i)} \pm \eta
$$

Proof. The general strategy to prove this lemma will be as follows: we first write the constraints given by ineq. (5.5) (regarding the intersection profile of $\left\{V_{i}\right\}_{i=1}^{k}$ ) as a system of linear inequalities, which, by hypothesis, has no solution. Hence, by a variant of Farka's lemma, we show there is a linear combination of such inequalities that witnesses the infeasibility of the system. We then show that a typical sample "inherits" a similar linear combination, which by its turn witnesses the infeasibility of a system of inequalities related to (5.6).

Suppose $V$ has no partition satisfying ineq. (5.5) and consider the following system $\mathcal{S}_{V}$ of linear inequalities (on $n k$ variables $x_{j}^{(i)}, i \in[k], j \in[n]$ ).

\section{System of inequalities $\mathcal{S}_{V}$}

$$
\begin{array}{lrl}
\forall r \in[t], \forall i \in[k]: & \left(\alpha_{r}^{(i)}-2 \eta\right) n \leq \sum_{j \in S_{r}} x_{j}^{(i)} \leq\left(\alpha_{r}^{(i)}+2 \eta\right) n \\
\forall j \in[n]: & x_{j}^{(1)} \geq 0, \ldots, x_{j}^{(k)} \geq 0, \sum_{i=1}^{k} x_{j}^{(i)}=1
\end{array}
$$

Note: we named the inequalities related with the intersection profile as $\left(I_{r, i}\right)$ and the ones related with $\left\{x_{j}^{(i)}\right\}$ specifying a partition as $\left(P_{j}\right)$. Also, the last equality from $\left(P_{j}\right)$ should be formally considered as two inequalities.

Claim 5.4. The system $\mathcal{S}_{V}$ has no solution.

Proof. Indeed, if there is a solution for $\mathcal{S}_{V}$, then consider a "basic solution" $\left\{x_{j}^{(i)}\right\}_{j \in[n]}^{i \in[k]}$. We recall that a basic solution (see e.g. [BT97, Theorem 2.3]) for $\mathcal{S}_{V}$ is one in which at least $n k$ linearly independent (l.i.) inequalities ${ }^{1}$ are active, i.e., are satisfied with equality.

For each $j \in[n]$, it cannot be that every inequality from $P_{j}$ is active. In fact, either $k$ l.i. inequalities from $P_{j}$ are active - in that case every $x_{j}^{(i)} \in\{0,1\}$ for every $i \in[k]$ - or less than $k$ l.i. inequalities from $P_{j}$ are active - in that case there must be some fractional $x_{j}^{(i)}$. In any case, a solution has a total of at most $t k$ active l.i. inequalities from $I_{r, i}$.

Let $B$ be the set of vertices $j \in[n]$ for which less than $k$ l.i. inequalities are active. We must have

$$
n k \leq \#\left\{\begin{array}{c}
\text { l.i. active } \\
\text { inequalities }
\end{array}\right\} \leq|B|(k-1)+(n-|B|) k+t k=n k-|B|+t k,
$$

which implies $|B| \leq t k \leq \eta n$.

\footnotetext{
${ }^{1} \mathrm{~A}$ set $\left\{u_{k}^{t} x \leq b_{k}\right\}_{i=1}^{m}$ of inequalities is linearly independent if the coefficient vectors $\left\{u_{k}\right\}_{i=1}^{m}$ are linearly independent
} 
Finally, consider the partition $\left\{V_{i}^{\prime}\right\}_{i=1}^{k}$ of $V \backslash B$ with $V_{i}^{\prime}=\left\{j: x_{j}^{(i)}=1\right\}$. Such a partition has intersection profile $\left\{\alpha_{r}^{(i)} \pm 2 \eta\right\}_{r}^{i}$. After distributing the vertices of $B$ arbitrarily, one gets a partition $\left\{V_{i}\right\}_{i=1}^{k}$ with intersection profile $\left\{\alpha_{r}^{(i)} \pm 3 \eta\right\}_{r}^{i}$, i.e., a partition that satisfies ineq. (5.5).

Now, let $Q \in_{\mathrm{U}}\left(\begin{array}{c}V \\ q\end{array}\right)$. Consider the following system of inequalities on $q k$ variables $\left\{y_{j}^{(i)}\right\}_{j \in Q}^{i \in[k]}$.

\section{System of inequalities $\mathcal{S}_{Q}$}

$$
\begin{array}{r}
\forall r \in[t], \forall i \in[k]: \quad\left(\alpha_{r}^{(i)}-\eta\right) q \leq \sum_{j \in S_{r}} y_{j}^{(i)} \leq\left(\alpha_{r}^{(i)}+\eta\right) q \\
\forall j \in[q]: \quad y_{j}^{(1)} \geq 0, \ldots, y_{j}^{(k)} \geq 0, \sum_{i=1}^{k} y_{j}^{(i)}=1
\end{array}
$$

Note that if $Q$ has an equipartition $\left\{Q_{i}\right\}_{i=1}^{k}$ satisfying ineq.(5.6), then one can produce a solution to $\mathcal{S}_{Q}$ by setting $y_{j}^{(i)}=1$ if $j \in Q_{i}$, and $y_{j}^{(i)}=0$ otherwise. Hence, in order to prove Lemma 5.3, it suffices to upper bound the probability that $\mathcal{S}_{Q}$ has a solution by $e^{-q \eta^{2} / 2}$. For that, we first prove the following consequence of the infeasibility of $\mathcal{S}_{V}$.

Claim 5.5. Since $\mathcal{S}_{V}$ has no solution, there must be a non-negative linear combination of the constraints in $\bigcup_{r, i} I_{r, i}$ that has no solution satisfying the constraints in $\bigcup_{j} P_{j}$. Moreover we can assume that, for each pair $(r, i)$, such a linear combination uses either the lower or the upper bound of $I_{r, i}$.

Proof. Indeed, write all inequalities $I_{r, i}$ in $\mathcal{S}_{V}$ as $A x \leq b$, for some $A \in \mathbb{R}^{2 t k \times n k}$ and $b \in \mathbb{R}^{2 t k}$, and also let $P=\left\{x \in \mathbb{R}^{n k}: x\right.$ satisfies $\left.\bigcup_{j \in[n]} P_{j}\right\}$. If $\mathcal{S}_{V}$ has no solution, then the sets $\{A x: x \in P\} \subset \mathbb{R}^{2 t k}$ and $\left\{b^{\prime}: b^{\prime} \leq b\right\} \subset \mathbb{R}^{2 t k}$ have no intersection. Since the first set is compact, for it is the image of a compact set under a linear transformation, and the second set is closed there must be a hyperplane that strictly separates them (see e.g. [BT97, Hyperplane Separation Theorem]). In other words, there must be $u \in \mathbb{R}^{2 t k}$ and $r \in \mathbb{R}$ such that

$$
u^{T}(A x)>u^{T} b^{\prime}, \quad \text { for every } x \in P \text { and } b^{\prime} \leq b
$$

Also, note we must have $u \geq 0$ (for if $u_{\ell}<0$, one could make $u^{T} b^{\prime}$ arbitrarily large by choosing a sufficiently negative $\left.b_{\ell}^{\prime}\right)$. Hence, $\left(u^{T} A\right) x \leq u^{T} b$ is a non-negative linear combination of the inequalities $A x \leq b$. On the other hand, every $x \in P$ satisfies

$$
\left(u^{T} A\right) x=u^{T}(A x)>u^{T} b,
$$

which violates that linear combination. Next, we proceed to the "moreover" assertion of the claim.

For a given $(r, i) \in[t] \times[k]$, let $\ell, \ell^{\prime} \in[2 t k]$ be the indices associated with the upper and lower bound restrictions of $I_{r, i}$. We will show we can assume that either $u_{\ell}=0$ or $u_{\ell^{\prime}}=0$, i.e., that the linear combination given by $u$ uses either the upper or the lower bound. Let $e_{\ell, \ell^{\prime}} \in \mathbb{R}^{2 t k}$ be the vector $\left(\delta_{j, \ell}+\delta_{j, \ell^{\prime}}\right)_{j=1}^{2 t k}$, i.e., the vector with entries $\ell$ and $\ell^{\prime}$ equal to 1 and all other entries zero. Given a vector $u \in \mathbb{R}^{2 t k}$ as above, define $\bar{u}=u-\mu e_{\ell, \ell^{\prime}} \geq 0$, where $\mu=\min \left\{u_{\ell}, u_{\ell^{\prime}}\right\}$. Then $\bar{u}$ is such that either $\bar{u}_{\ell}=0$ or $\bar{u}_{\ell^{\prime}}=0$. Moreover, since $\bar{u}^{T} A=u^{T} A-\mu e_{\ell, \ell^{\prime}}^{T} A=u^{T} A$ 
and $\bar{u}^{T} b=u^{T} b-\mu e_{\ell, \ell^{\prime}}^{T} b=u^{T} b-\mu 4 \eta \leq u^{T} b$, every $x \in P$ is such that $\left(\bar{u}^{T} A\right) x=\left(u^{T} A\right) x>u^{T} b \geq \bar{u}^{T} b$. Repeat this argument for every $(r, i)$ to get the claimed linear combination of $\bigcup I_{r, i}$.

From Claim 5.5 we can assume there are numbers $u_{r}^{(i)}$ — the sign of those numbers indicates which bound of the $I_{r, i}$ constraint is being used - such that by letting:

$$
c_{0}=\sum_{r=1}^{t} \sum_{i=1}^{k}\left(u_{r}^{(i)} \alpha_{r}^{(i)}+2\left|u_{r}^{(i)}\right| \eta\right) n
$$

and

$$
c_{j}^{(i)}=\sum_{r: j \in S_{r}} u_{r}^{(i)}
$$

we get that

$$
\sum_{j=1}^{n} \sum_{i=1}^{k} c_{j}^{(i)} x_{j}^{(i)} \leq c_{0} \quad \text { has no solution satisfying } \bigcup_{j} P_{j} .
$$

But this happens if and only if

$$
\sum_{j=1}^{n} \min _{i \in[k]} c_{j}^{(i)}>c_{0}
$$

since $\sum_{j=1}^{n} \sum_{i=1}^{k} c_{j}^{(i)} x_{j}^{(i)}$ is minimized (over $\left\{x_{j}^{(i)}\right\}$ satisfying $P$ ) by simply setting (for every $j \in$ [n]) $x_{j}^{(i)}=1$ for some $i=\operatorname{argmin}_{i \in[k]} c_{j}^{(i)}$ and $x_{j}^{(i)}=0$ otherwise. We have thus arrived at a single equation (ineq. (5.7)) that witnesses $\mathcal{S}_{V}$ has no solution.

Now, suppose there is a solution $\left\{y_{j}^{(i)}\right\}$ to $\mathcal{S}_{Q}$. By taking a $u_{r}^{(i)}$-linear combination of the inequalities $I_{r, i}^{\prime}$, we get

$$
\begin{aligned}
\sum_{i \in Q} \sum_{i=1}^{k} c_{j}^{(i)} y_{j}^{(i)} \leq \sum_{r=1}^{t} \sum_{i=1}^{k}\left(u_{r}^{(i)} \alpha_{r}^{(i)}+\left|u_{r}^{(i)}\right| \eta\right) q & =\sum_{r=1}^{t} \sum_{i=1}^{k}\left(u_{r}^{(i)} \alpha_{r}^{(i)}+2\left|u_{r}^{(i)}\right| \eta-\left|u_{r}^{(i)}\right| \eta\right) q \\
& =c_{0} \frac{q}{n}-\sum_{r=1}^{t} \sum_{i=1}^{k}\left|u_{r}^{(i)}\right| \eta q .
\end{aligned}
$$

By putting $M=\sum_{r=1}^{t} \sum_{i=1}^{k}\left|u_{r}^{(i)}\right|$, we get

$$
\sum_{i \in Q} \sum_{i=1}^{k} c_{j}^{(i)} y_{j}^{(i)} \leq c_{0} \frac{q}{n}-M \eta q
$$

which again, by the $\left(P_{j}\right)$ constraints on $y_{j}^{(i)}$, implies $Q$ must be such that

$$
\sum_{j \in Q} \min _{i \in[k]} c_{j}^{(i)} \leq \frac{q}{n} c_{0}-M \eta q
$$

Define $Y_{j}=\min _{i \in[k]} c_{j}^{(i)}$ for every $j \in[n]$, the expectation of $\sum_{j \in Q} Y_{j}$ is

$$
\mathbb{E}\left(\sum_{j \in Q} Y_{j}\right)=\mathbb{E}\left(\sum_{j=1}^{n} Y_{j} \mathbb{1}_{j \in Q}\right)=\frac{q}{n} \sum_{j=1}^{n} Y_{j}>\frac{q}{n} c_{0}
$$

where the last inequality follows from ineq. (5.7). By that bound on the expectation of $\sum_{j \in Q} Y_{j}$ and 
the fact that $\left|Y_{j}\right| \leq M$ for each $j$, Lemma 2.4 yields

$\mathbb{P}\left(\sum_{j \in Q} \min _{i \in[k]} c_{j}^{(i)} \leq \frac{q}{n} c_{0}-M \eta q\right) \leq \mathbb{P}\left(\sum_{j=1}^{q} Y_{j} \leq \mathbb{E}\left(\sum_{j \in Q} Y_{j}\right)-M \eta q\right) \leq \exp \left\{\frac{-2(M \eta q)^{2}}{q(2 M)^{2}}\right\}=e^{-q \eta^{2} / 2}$

Hence, with probability at least $1-e^{-q \eta^{2} / 2}$ the sample $Q$ is unable to satisfy ineq. (5.8). In that case, there cannot be a solution to $\mathcal{S}_{Q}$, as required.

As a consequence of Lemma 5.3, we have the following result.

Lemma 5.6. For every $\eta>0$ and any positive integers $k$, $t$ and $a$, there is $q=32\left\lceil a / \eta^{2}+2 k t /\left(\eta^{3}\right)\right\rceil$ satisfying the following. Let $S_{1}, \ldots, S_{t}$ be subsets of vertices of set $V$ and let $Q$ be a subset of $V$ chosen uniformly at random. Then, with probability at least $1-e^{-a}$, for every partition $\left\{Q_{i}\right\}_{i=1}^{k}$ of $Q$, there is a partition $\left\{V_{i}\right\}_{i=1}^{k}$ of $V$ such that

$$
\frac{1}{n}\left|V_{i} \cap S_{r}\right|=\frac{1}{q}\left|Q_{i} \cap S_{r}\right| \pm \eta
$$

and, likewise, for every partition $\left\{V_{i}\right\}_{i=1}^{k}$ of $V$, there is a partition $\left\{Q_{i}\right\}_{i=1}^{k}$ of $Q$ satisfying ineq. (5.9).

Proof. Consider the following collection of intersection profiles of integer multiples of $\eta / 2$.

$$
\mathcal{N}=\left\{\left(\alpha_{r}^{(i)}\right)_{i \in[k], r \in t}: \alpha_{r}^{(i)}=u_{r}^{(i)} \eta / 4, \text { for some integer } 0 \leq u_{r}^{(i)} \leq 2 / \eta\right\}
$$

We have $|\mathcal{N}| \leq(1+2 / \eta)^{k t} \leq e^{2 k t / \eta}$ For each element of $\mathcal{N}$, apply Lemma 5.3 for the family $\left\{S_{1}, \ldots, S_{t}\right\}$ with error parameter $\eta / 4$. With probability at least

$$
1-|\mathcal{N}| e^{-q \eta^{2} / 32} \geq 1-e^{2 k t / \eta} \cdot e^{-a-2 k t / \eta}=1-e^{-a}
$$

over the choice of $Q$, the assertion of Lemma 5.3 holds simultaneously for every element of $\mathcal{N}$.

Let $\left\{Q_{i}\right\}_{i=1}^{k}$ be any partition of $Q$. From the very definition of $\mathcal{N}$ there must be $\left(\alpha_{r}^{(i)}\right) \in \mathcal{N}$ for which

$$
\forall i \in[k], \forall r \in[t]: \frac{1}{q}\left|Q_{i} \cap S_{r}\right|=\alpha_{r}^{(i)} \pm \eta / 4 .
$$

By Lemma 5.3, there must be a partition of $\left\{V_{i}\right\}_{i=1}^{k}$ of $V$ such that

$$
\forall i \in[k], \forall r \in[t]: \frac{1}{n}\left|V_{i} \cap S_{r}\right|=\alpha_{r}^{(i)} \pm 3 \eta / 4=\frac{1}{q}\left|Q_{i} \cap S_{r}\right| \pm \eta
$$

as desired. The other direction of the lemma is an application of Lemma 2.5.

\subsection{Proof of Theorem 5.1}

We begin with the following lemma, which roughly asserts that for every graph $G$, there are sets $\left\{S_{r}\right\}_{r=1}^{t}$ of vertices for which one can estimate the number of edges e $(A, B)$ between any two sets $A, B \subseteq V(G)$ by just computing the intersections $\left|A \cap S_{r}\right|$ and $\left|B \cap S_{r}\right|$ - in particular, one can (approximately) recover every density $\mathrm{d}\left(V_{i}, V_{j}\right)$ between classes of a partition $\left\{V_{i}\right\}_{i=1}^{k}$ from its intersection profile on $\left\{S_{r}\right\}_{r=1}^{t}$. Moreover, it also states that a typical sample of $G$ also satisfy this statement (with respect to the same sets $\left\{S_{r}\right\}_{r=1}^{t}$ ). 
Lemma 5.7. For every $\gamma>0$ and $\delta>0$, there are integers $T=T_{5.7}(\gamma)=\operatorname{poly}\left(\gamma^{-1}\right)$ and $q=$ $q_{5.7}(\gamma, \delta)=\operatorname{poly}\left(\frac{1}{\gamma}, \log \frac{1}{\delta}\right)$ satisfying the following. For every graph $G=(V, E)$, with $|V| \geq q$, there are sets $S_{1}, \ldots, S_{t} \subset V(G)$ and real numbers $d_{1}, \ldots, d_{t}$ (with $t \leq T$ ) for which

$$
\mathrm{e}(A, B)=\sum_{i=1}^{t} d_{i} \cdot\left|A \cap S_{i}\right|\left|B \cap S_{i}\right| \pm \gamma n^{2}
$$

for every $A, B \subseteq V$.

Moreover, if $Q \in\left(\begin{array}{c}V \\ q\end{array}\right)$ is a subset of $q$ vertices chosen uniformly at random, then with probability at least $1-\delta$,

$$
\mathrm{e}(A, B)=\sum_{i=1}^{t} d_{i} \cdot\left|A \cap S_{i}\right|\left|B \cap S_{i}\right| \pm \gamma q^{2}
$$

for every $A, B \subseteq Q$.

Proof. Let $q$ and $\gamma^{\prime}$ be as given by Theorem 3.9 with inputs $\gamma$ and $\delta$ and let $T$ be as in Lemma 3.14 with input $\gamma$. Let $G$ and $Q$ be as in the statement of the lemma. By Lemma 3.14 there are sets $\left\{S_{r}\right\}_{r=1}^{t}$, numbers $\left\{d_{r}\right\}_{r=1}^{t}$ and a weighted graph $\Delta$, with $\|\Delta\|_{\square} \leq \gamma^{\prime} \leq \gamma$, for which

$$
\mathrm{e}(A, B)=\sum_{i=1}^{t} d_{i} K_{S_{i}}(A, B)+\Delta(A, B)=\sum_{i=1}^{t} d_{i}\left|A \cap S_{i}\right|\left|B \cap S_{i}\right| \pm \gamma^{\prime} n^{2},
$$

for every $A, B \subseteq V$.

Moreover, for every $A, B \subseteq Q$, we have (with probability at least $1-\delta$ ),

$$
\mathrm{e}(A, B)=\sum_{i=1}^{t} d_{i} K_{S_{i}}(A, B)+\Delta[Q](A, B)=\sum_{i=1}^{t} d_{i}\left|A \cap S_{i}\right|\left|B \cap S_{i}\right| \pm \gamma n^{2}
$$

where the last (in)equality follows from Theorem 3.9.

Lemma 5.8. For every $\zeta>0, \delta>0$ and every integer $k>0$, there is $q=q_{5.8}(\zeta, k, \delta)=$ $\operatorname{poly}\left(\zeta, k, \log \frac{1}{\delta}\right)$ satisfying the following. Let $Q \in_{U}\left(\begin{array}{l}V \\ q\end{array}\right)$ be a set of $q$ vertices taken uniformly at random from a graph $G$ of size $n=|V(G)|$. Then, with probability at least $1-\delta$, for every partition $\left\{Q_{i}\right\}_{i=1}^{k}$ of $G[Q]$, there is a partition $\left\{V_{i}\right\}_{i=1}^{k}$ of $G$ satisfying

(i) $\frac{1}{n}\left|V_{i}\right|=\frac{1}{q}\left|Q_{i}\right| \pm \zeta ;$

(ii) $\frac{1}{n^{2}} \mathrm{e}\left(V_{i}, V_{j}\right)=\frac{1}{q^{2}} \mathrm{e}\left(Q_{i}, Q_{j}\right) \pm \zeta$.

Proof. Put

$$
\gamma=\frac{1}{3} \zeta, \quad D=D_{3.14}, \quad T=T_{5.7}(\gamma), \quad \eta=\gamma /(3 \sqrt{D T}), \quad q=\max \left\{100 k T \log \frac{1}{\delta} / \eta^{3}, q_{5.7}\left(\gamma, \frac{1}{2} \delta\right)\right\}
$$

and let $\left\{d_{r}\right\}_{r=1}^{t}$ be real numbers and $\left\{S_{r}\right\}_{r=1}^{t}$ be sets of vertices of $G$ satisfying ineq. (5.10) from Lemma 5.7.

With probability at least $1-\frac{1}{2} \delta$, the set $Q \in\left(\begin{array}{c}V(G) \\ q\end{array}\right)$, taken uniformly at random, satisfies ineq. (5.11) from Lemma 5.7. With probability at least $1-\frac{1}{2} \delta-\frac{1}{2} \delta=1-\delta, Q$ also satisfies the assertion of Lemma 5.6 with respect to the following sets of vertices: $\left\{S_{r}\right\}_{i=1}^{t} \cup\{V(G)\}$. In particular, 
for every partition $\left\{Q_{i}\right\}_{i=1}^{k}$ of $Q$, there exists a partition $\left\{V_{i}\right\}_{i=1}^{k}$ of $G$ for which

$$
\frac{\left|V_{i}\right|}{n}=\frac{\left|Q_{i}\right|}{q} \pm \eta=\frac{\left|Q_{i}\right|}{q} \pm \zeta
$$

for every $1 \leq i \leq k$ - which already sets one of the requirements of the lemma - and

$$
\frac{\left|V_{i} \cap S_{r}\right|}{n}=\frac{\left|Q_{i} \cap S_{r}\right|}{q} \pm \eta
$$

for every $1 \leq r \leq t$ and $1 \leq i \leq k$.

For every $1 \leq i, j \leq k$, we have

$$
\begin{aligned}
\frac{1}{n^{2}} \mathrm{e}\left(V_{i}, V_{j}\right) \pm \gamma & \stackrel{(5.10)}{=} \sum_{r=1}^{t} d_{r} \cdot \frac{\left|S_{r} \cap V_{i}\right|}{n} \cdot \frac{\left|S_{r} \cap V_{j}\right|}{n} \\
& \stackrel{(5.13)}{=} \sum_{r=1}^{t} d_{r} \cdot \frac{\left|S_{r} \cap Q_{i}\right| \pm \eta q}{q} \cdot \frac{\left|S_{r} \cap Q_{j}\right| \pm \eta q}{q} \\
& =\frac{1}{q^{2}} \sum_{r=1}^{t}\left(d_{r} \cdot\left|S_{r} \cap Q_{i}\right| \cdot\left|S_{r} \cap Q_{j}\right| \pm 3\left|d_{r}\right| \eta q^{2}\right) \\
& \stackrel{(5.11)}{=} \frac{1}{q^{2}} \mathrm{e}\left(Q_{i}, Q_{j}\right) \pm \gamma \pm 3 \eta \sum_{r=1}^{t}\left|d_{r}\right| .
\end{aligned}
$$

Recall that Lemma 5.7 tells us that $\sum_{r=1}^{t} d_{r}^{2} \leq D$. Applying Cauchy-Schwarz, we get that

$$
\begin{aligned}
\frac{1}{n^{2}} \mathrm{e}\left(V_{i}, V_{j}\right) & =\frac{1}{q^{2}} \mathrm{e}\left(Q_{i}, Q_{j}\right) \pm \gamma \pm 3 \eta \sqrt{D t} \\
& =\frac{1}{q^{2}} \mathrm{e}\left(Q_{i}, Q_{j}\right) \pm 3 \gamma
\end{aligned}
$$

By our choice of $\gamma$, we get that $\frac{1}{n^{2}} \mathrm{e}\left(V_{i}, V_{j}\right)=\frac{1}{q^{2}} \mathrm{e}\left(Q_{i}, Q_{j}\right) \pm \zeta$.

Proof of Theorem 5.1. We will only prove item (i) from Theorem 5.1, since the assertion from item (ii) follows by a symmetric argument. Moreover, we also note that the assertion from item (ii) is actually much easier to prove than the one from item (i) from Lemma 2.5.

Given a graph $G$, apply Lemma 5.8 with $k, \delta$ and $\zeta=\min \left\{\varepsilon / 2 k^{2}, \varepsilon / 8 k\right\}$. Then with probability at least $1-\delta$, for every equipartition $\left\{Q_{i}\right\}_{i=1}^{k}$ of $G[Q]$ there is a partition $\left\{W_{i}\right\}_{i=1}^{k}$ of $G$ satisfying

(i) $\frac{1}{n}\left|W_{i}\right|=\frac{1}{q}\left|Q_{i}\right| \pm \varepsilon / 8 k=\frac{1}{k} \pm \varepsilon / 8 k$.

(ii) $\frac{1}{n^{2}} \mathrm{e}\left(W_{i}, W_{j}\right)=\frac{1}{q^{2}} \mathrm{e}\left(Q_{i}, Q_{j}\right) \pm \varepsilon / 2 k^{2}$.

Let $\left\{V_{i}\right\}_{i=1}^{k}$ be an equipartition of $G$ obtained from $\left\{W_{i}\right\}_{i=1}^{k}$ after arbitrarily redistributing up to $\frac{1}{8} \varepsilon(n / k)$ vertices from each class $W_{i}$. Since,

$$
\left|\mathrm{e}\left(V_{i}, V_{j}\right)-\mathrm{e}\left(W_{i}, W_{j}\right)\right| \leq \frac{\varepsilon n}{8 k} \max \left\{\left|V_{i}\right|,\left|W_{i}\right|\right\}+\frac{\varepsilon n}{8 k} \max \left\{\left|V_{j}\right|,\left|W_{j}\right|\right\} \leq \frac{\varepsilon n}{4 k}\left(\frac{n}{k}+\frac{\varepsilon n}{8 k}\right) \leq \frac{\varepsilon n^{2}}{2 k^{2}},
$$

we must have

$$
\mathrm{d}\left(V_{i}, V_{j}\right)=\frac{k^{2} \mathrm{e}\left(V_{i}, V_{j}\right)}{n^{2}}=\frac{k^{2} \mathrm{e}\left(W_{i}, W_{j}\right)}{n^{2}} \pm \frac{1}{2} \varepsilon=\frac{k^{2} \mathrm{e}\left(Q_{i}, Q_{j}\right)}{n^{2}} \pm k^{2}\left(\frac{1}{2} \varepsilon / k^{2}\right) \pm \frac{1}{2} \varepsilon=\mathrm{d}\left(Q_{i}, Q_{j}\right) \pm \varepsilon,
$$


as desired.

\subsection{Proof of Theorem 5.2}

As mentioned in the beginning of this chapter, the proof of Theorem 5.2 is also based on Lemma 5.6, but we will need a richer family $\left\{S_{r}\right\}_{r=1}^{t}$ on which we will compute the intersection pattern of a partition. More specifically, we will need a lemma similar to Lemma 5.7 but concerning the number of homomorphic copies of $C_{4}=\mathbb{Z}_{0}^{\circ}$ between the pair $(A, B)$, instead of the number of edges between $(A, B)$. Given a graph $G=(V, E)$ and sets $A, B \subseteq V$, let $\operatorname{Hom}_{C_{4}}(A, B)=\ddot{\mathrm{t}}_{C_{4}}(A, B)|A|^{2}|B|^{2}$ be the number of homomorphic copies of $C_{4}=\mathfrak{Z}_{0}^{\circ}$ between $A$ and $B$.

Lemma 5.9. For every $\gamma>0$, there are integers $T=T_{5.9}(\gamma)=\operatorname{poly}(1 / \gamma)$ and $q_{5.9}(\gamma)=$ $\operatorname{poly}(1 / \gamma)$ satisfying the following. For every graph $G=(V, E)$, with $|V| \geq \exp \left\{\Omega\left(\gamma^{-1}\right)\right\}$, there are sets $\widehat{S}_{1}, \widehat{R}_{1}, \ldots, \widehat{S}_{t}, \widehat{R}_{t} \subset V(G)$ and real numbers $\widehat{d}_{1}, \ldots, \widehat{d}_{t}$ with $t \leq T$ satisfying

$$
\operatorname{Hom}_{C_{4}}(A, B)=\sum_{i=1}^{t} \widehat{d}_{i} \cdot\left|A \cap \widehat{S}_{i}\right|^{2}\left|B \cap \widehat{R}_{i}\right|^{2} \pm \gamma n^{4}
$$

for every $A, B \subseteq V(G)$.

Moreover, if $Q \in\left(\begin{array}{l}V \\ q\end{array}\right)$ is a subset of $q \geq q_{5.9}$ vertices chosen uniformly at random, then

$$
\operatorname{Hom}_{C_{4}}(A, B)=\sum_{i=1}^{t} \widehat{d}_{i} \cdot\left|A \cap \widehat{S}_{i}\right|^{2}\left|B \cap \widehat{R}_{i}\right|^{2} \pm \gamma q^{4},
$$

for every $A, B \subseteq Q$, with probability at least $9 / 10$.

Finally, one can assume that $\sum_{i=1}^{t}\left(\widehat{d}_{i}\right)^{2} \leq D_{5.9}$ for some absolute constant $D_{5.9}$.

Proof. Given a graph $G$, we define the array $A_{G} \in\{0,1\}^{V^{4}}$ by putting $A_{G}(x, y, z, w)=1$ if $x y z w$ is a 4-cycle of $G$ (in this order), and $A_{G}(x, y, z, w)=0$ otherwise. We say that $A_{G}$ is "symmetric" in the sense that $A_{G}$ is invariant over the (bipartite) automorphisms of $K_{2,2}$. More generally, we define a matrix $A \in \mathbb{R}^{V^{4}}$ to be symmetric if

$$
A(x, y, z, w)=A(z, y, x, w)=A(z, w, x, y)=A(x, w, z, y)
$$

for every $(x, y, z, w) \in V^{4}$. Given a number $\widehat{d} \in \mathbb{R}$ and two sets $\widehat{S}, \widehat{R} \subseteq V$, denote by $\widehat{d} \cdot K_{\widehat{S}, \widehat{R}}$ the array $A \in \mathbb{R}^{V^{4}}$ such that $A(x, y, z, w)=\widehat{d}$ if $(x, y, z, w) \in \widehat{S} \times \widehat{R} \times \widehat{S} \times \widehat{R}$, and $A(x, y, z, w)=0$ otherwise.

Given any symmetric array $A \in \mathbb{R}^{V^{4}}$ and any four sets $X, Y, Z, W \subseteq V$, we will denote by $A(X, Y, Z, W)$ the sum $\sum A(x, y, z, w)$ over all $(x, y, z, w) \in X \times Y \times Z \times W$. We also define the norms

$$
\|A\|_{\square}=\frac{1}{|V|^{4}} \max _{X, Y \subseteq V}|A(X, Y, X, Y)| \quad \text { and } \quad\|A\|_{\square^{\prime}}=\frac{1}{|V|^{4}} \max _{X, Y, Z, W \subseteq V}|A(X, Y, Z, W)| .
$$

It is easy to check that, by the symmetry of $A$ and by the inclusion-exclusion principle, we have $\frac{1}{4}\|A\|_{\square^{\prime}} \leq\|A\|_{\square} \leq\|A\|_{\square^{\prime}}$.

Alon, de la Vega, Kannan and Karpinski proved in [AFdlVKK03, Theorem 4] a statement analogous to Lemma 3.13 for arrays of arbitrary dimension. In particular, one can apply their 
result to $A_{G}$. By using the fact that $A_{G}$ is symmetric and proceeding analogously to the proof of Lemma 3.14, we get the following corollary: for every $\gamma^{\prime}>0$ there exist pairs of sets of vertices $\left\{\widehat{S}_{i}, \widehat{R}_{i}\right\}_{i=1}^{t}$ and real numbers $\left\{\widehat{d}_{i}\right\}_{i=1}^{t}$, with $t \leq T=\operatorname{poly}\left(1 / \gamma^{\prime}\right)$, for which

$$
A_{G}=\sum_{i=1}^{t} \widehat{d}_{i} \cdot K_{\widehat{S}_{i}, \widehat{R}_{i}}+\Delta,
$$

with $\|\Delta\|_{\square} \leq \gamma^{\prime}$ and $\sum_{i=1}^{t}\left(\widehat{d}_{i}\right)^{2} \leq D$ for some absolute constant $D$.

Now, let $Q \in_{\mathrm{U}}\left(\begin{array}{l}V \\ q\end{array}\right)$ be chosen uniformly at random. By restricting Eq. (5.16) to the subarray spanned by $Q^{4}$, we get

$$
\left.A_{G}\right|_{Q^{4}}=\sum_{i=1}^{t} \widehat{d}_{i} \cdot K_{\widehat{S}_{i} \cap Q, \widehat{R}_{i} \cap Q}+\left.\Delta\right|_{Q^{4}} .
$$

By [AFdlVKK03, Theorem 6], there are absolute constants $C_{1}, C_{2}>0$ such that, for every $\gamma^{\prime}>0$, if $q \geq C_{1} \frac{1}{\gamma^{\prime 4}} \log \frac{1}{\gamma^{\prime 2}}$ then $\left\|\left.\Delta\right|_{Q^{4}}\right\|_{\square} \leq C_{2} \gamma^{\prime}$ with probability at least $9 / 10$.

Finally, set $\gamma^{\prime}=\gamma / C_{2}$ and note that for every $A, B \subseteq V$, it follows from Eq. (5.16) that

$$
\begin{aligned}
\operatorname{Hom}_{C_{4}}(A, B)=A_{G}(A, B, A, B) & =\sum_{i=1}^{t} \widehat{d}_{i} \cdot K_{\widehat{S}_{i}, \widehat{R}_{i}}(A, B, A, B)+\Delta(A, B, A, B) \\
& =\sum_{i=1}^{t} \widehat{d}_{i}\left|A \cap \widehat{S}_{i}\right|^{2}\left|B \cap \widehat{R}_{i}\right|^{2} \pm\|\Delta\|_{\square}=\sum_{i=1}^{t} \widehat{d}_{i}\left|A \cap \widehat{S}_{i}\right|^{2}\left|B \cap \widehat{R}_{i}\right|^{2} \pm \gamma^{\prime}
\end{aligned}
$$

In particular, ineq. (5.14) holds. Ineq. (5.15) follows analogously from Eq. (5.17).

Proof of Theorem 5.2. As in the proof of Theorem 5.1, we only need to prove item (iii) from Theorem 5.2, since the assertion of item (iv) follows by a symmetric argument - and it is also much easier to prove on its own by concentration results. Also, it will be enough to prove that, with probability at least $2 / 3$, for every equipartition $\left\{Q_{i}\right\}_{i=1}^{k}$ of $G[Q]$, there is an equipartition $\left\{V_{i}\right\}_{i=1}^{k}$ of $G$ for which

$$
\mathrm{d}\left(V_{i}, V_{j}\right)=\mathrm{d}\left(Q_{i}, Q_{j}\right) \pm \varepsilon \quad \text { and } \quad \ddot{\mathrm{t}}_{C_{4}}\left(V_{i}, V_{j}\right)=\ddot{\mathrm{t}}_{C_{4}}\left(Q_{i}, Q_{j}\right) \pm \varepsilon
$$

for every $1 \leq i \leq j \leq k$. Indeed, by putting $\alpha=\operatorname{Reg}_{\mathfrak{a}_{0}}\left(Q_{i}, Q_{j}\right)$, we will then get that $\ddot{\mathrm{t}}_{C_{4}}\left(V_{i}, V_{j}\right) \leq \ddot{\mathrm{t}}_{C_{4}}\left(Q_{i}, Q_{j}\right)+\varepsilon=\left(\mathrm{d}\left(Q_{i}, Q_{j}\right)^{4}+\alpha\right)+\varepsilon \leq\left(\mathrm{d}\left(V_{i}, V_{j}\right)+\varepsilon\right)^{4}+\alpha+\varepsilon \leq \mathrm{d}\left(V_{i}, V_{j}\right)^{4}+(\alpha+16 \varepsilon)$, that is, that $\operatorname{Reg}_{g_{0}}\left(V_{i}, V_{j}\right) \leq \alpha+16 \varepsilon$, and we get the exact statement of the theorem by starting with a smaller $\varepsilon$. Put

$$
\gamma=\min \left\{\varepsilon / 6 k^{2}, \varepsilon / 8 k\right\}, \quad T=\max \left\{T_{5.7}(\gamma), T_{5.9}(\gamma)\right\}, \quad D=D_{5.9}, \quad \eta=\gamma /(15 \sqrt{D T})
$$

and

$$
q=\max \left\{10 k T(\gamma) / \eta^{3}, q_{5.7}\left(\gamma, \frac{1}{3} \delta\right), q_{5.9}\left(\gamma, \frac{1}{3} \delta\right)\right\}
$$

Let $\left\{d_{i}\right\}_{i=1}^{t}$ be real numbers and $\left\{S_{i}\right\}_{i=1}^{t}$ be sets of vertices of $G$ satisfying ineq. (5.10) from Lemma 5.7 with $t \leq T$. Let $\left\{\widehat{d}_{i}\right\}_{i=1}^{t}$ be real numbers and $\left\{\widehat{S}_{i}, \widehat{T}_{i}\right\}_{i=1}^{t}$ be pairs of sets of vertices of $G$ satisfying ineq. (5.14) from Lemma 5.9 with $t \leq T$ (note that we can assume for convenience that 
the numbers of $S_{i}$ and $\left(S_{i}, T_{i}\right)$ are the same).

With probability at least $1-\frac{2}{3} \delta$, the set $Q \in\left(\begin{array}{c}V(G) \\ q\end{array}\right)$, taken uniformly at random, satisfies both ineq. (5.11) and ineq. (5.15) from Lemma 5.7 and Lemma 5.9. With probability at least $1-\delta, Q$ also satisfies the assertion of Lemma 5.6 with respect to the following sets of vertices: $\left\{S_{i}, \widehat{S}_{i}, \widehat{R}_{i}\right\}_{i=1}^{t} \cup$ $\{V(G)\}$. In particular, for any fixed equipartition $\left\{Q_{i}\right\}_{i=1}^{k}$ of $Q$, there exists a partition $\left\{W_{i}\right\}_{i=1}^{k}$ of $G$ for which

$$
\begin{gathered}
\frac{\left|W_{i} \cap S_{r}\right|}{n}=\frac{\left|Q_{i} \cap S_{r}\right|}{q} \pm \eta, \quad \frac{\left|W_{i} \cap \widehat{S}_{r}\right|}{n}=\frac{\left|Q_{i} \cap \widehat{S}_{r}\right|}{q} \pm \eta, \quad \frac{\left|W_{i} \cap \widehat{R}_{r}\right|}{n}=\frac{\left|Q_{i} \cap \widehat{R}_{r}\right|}{q} \pm \eta \\
\text { and } \\
\frac{\left|W_{i}\right|}{n}=\frac{\left|Q_{i}\right|}{q} \pm \eta .
\end{gathered}
$$

By the proof of Theorem 5.1 we already have $\frac{1}{n^{2}} \mathrm{e}\left(W_{i}, W_{j}\right)=\frac{1}{q^{2}} \mathrm{e}\left(Q_{i}, Q_{j}\right) \pm \frac{1}{2} \varepsilon / k^{2}$. Moreover, for every $1 \leq i, j \leq k$, we also have

$$
\begin{aligned}
\frac{1}{n^{4}} \operatorname{Hom}_{C_{4}}\left(W_{i}, W_{j}\right) \pm \gamma & \stackrel{(5.14)}{=} \sum_{r=1}^{t} \widehat{d}_{r} \cdot \frac{\left|\widehat{S}_{r} \cap W_{i}\right|^{2}}{n^{2}} \cdot \frac{\left|\widehat{R}_{r} \cap W_{j}\right|^{2}}{n^{2}} \\
& \stackrel{(5.18)}{=} \frac{1}{q^{4}} \sum_{r=1}^{t} d_{r} \cdot\left(\left|S_{r} \cap Q_{i}\right| \pm \eta q\right)^{2} \cdot\left(\left|R_{r} \cap Q_{j}\right| \pm \eta q\right)^{2} \\
& =\frac{1}{q^{4}} \sum_{r=1}^{t}\left(\widehat{d}_{r} \cdot\left|S_{r} \cap Q_{i}\right|^{2} \cdot\left|R_{r} \cap Q_{j}\right|^{2} \pm 15 \eta q^{4}\right) \\
& \stackrel{(5.15)}{=} \frac{1}{q^{4}} \operatorname{Hom}_{C_{4}}\left(Q_{i}, Q_{j}\right) \pm \gamma \pm 15 \eta \sum_{r=1}^{t}\left|\widehat{d}_{r}\right| .
\end{aligned}
$$

Recall that Lemma 5.9 tells us that $\sum_{r=1}^{t}\left(\widehat{d}_{r}\right)^{2} \leq D$. Applying Cauchy-Schwarz, we get that

$$
\begin{aligned}
\frac{1}{n^{4}} \operatorname{Hom}_{C_{4}}\left(W_{i}, W_{j}\right) & =\frac{1}{q^{4}} \operatorname{Hom}_{C_{4}}\left(Q_{i}, Q_{j}\right) \pm 2 \gamma \pm 15 \eta \sqrt{D t} \\
& =\frac{1}{q^{4}} \operatorname{Hom}_{C_{4}}\left(Q_{i}, Q_{j}\right) \pm 3 \gamma .
\end{aligned}
$$

By our choice of $\gamma$, we get that $\frac{1}{n^{4}} \operatorname{Hom}_{C_{4}}\left(W_{i}, W_{j}\right)=\frac{1}{q^{4}} \operatorname{Hom}_{C_{4}}\left(Q_{i}, Q_{j}\right) \pm \frac{1}{2} \varepsilon / k^{2}$.

Since, by (5.19), $\left|W_{i}\right|=n\left(\left|Q_{i}\right| / q \pm \eta\right)=\frac{n}{k} \pm \eta n=\frac{n}{k} \pm \frac{1}{8} \varepsilon \frac{n}{k}$, we can get an equipartition $\left\{V_{i}\right\}_{i=1}^{k}$ of $V(G)$ by redistributing up to $\frac{1}{8} \varepsilon(n / k)$ vertices from each class $W_{i}$. By a similar argument as in the proof of Theorem 5.1, we have both

$$
\mathrm{d}\left(V_{i}, V_{j}\right)=\mathrm{d}\left(Q_{i}, Q_{j}\right) \pm \varepsilon \quad \text { and } \quad \ddot{\mathrm{t}}_{C_{4}}\left(V_{i}, V_{j}\right)=\ddot{\mathrm{t}}_{C_{4}}\left(Q_{i}, Q_{j}\right) \pm \varepsilon
$$

for every $1 \leq i \leq j \leq k$.

\subsection{Applications}

A straightforward application of Theorem 5.1 allows one to test a rather general class of graph properties - namely the property of graphs having an equipartition into $k$ classes with given set 
of densities $\left\{d_{i j}: 1 \leq i \leq j \leq k\right\}$. Such properties were proved to be testable before by Goldreich, Goldwasser and Ron in [GGR98]; they then used their result to prove that many other interesting graph properties are also testable. In the following chapter, we show one can test such properties with a better sample complexity, as a consequence of Theorem 5.1. Then, we also apply Theorem 5.2 to test a generalization of such properties, in which we require the pair of classes of the equipartition to satisfy certain regularity constraints as well.

Chapters 8 and 9 can also be seen, in a way, as more convoluted applications of Theorem 5.1, which provide a way of efficiently estimating certain parameters. The main idea consists of showing that such parameters can be approximated by just having access to the set of quotients of the input graph into partitions of bounded size; see Chapter 7. 


\section{Chapter 6}

\section{Efficient testing of partition problems}

As discussed in Section 5.5, one can rederive (with a better sample complexity) a rather general result of [GGR98] concerning the property of graphs having a partition into $k$ classes with specified densities between the classes. Here, we extend their result by proving that the property $\Pi_{k, \bar{d}, \bar{\gamma}}$ below — which also imposes constraints on how regular each pair of classes should be - is testable.

Definition 6.1 $\left(\Pi_{k, \bar{d}, \bar{\gamma}}\right)$. Given an integer $k>0$, a collection $\bar{d}=\left\{d_{i j}: 1 \leq i \leq j \leq k\right\}$ of numbers $0 \leq d_{i j} \leq 1$ and a collection $\bar{\gamma}=\left\{\gamma_{i j}: 1 \leq i \leq j \leq k\right\}$ of numbers $0<\gamma_{i j} \leq 1$, one can consider the graph property $\Pi_{k, \bar{d}, \bar{\gamma}}$ of $n$-vertex graphs having an equipartition $\mathcal{V}=\left\{V_{i}\right\}_{i=1}^{k}$, where $\left(V_{i}, V_{j}\right)$ is $\gamma_{i j}$-quad-regular with density $\mathrm{d}\left(V_{i}, V_{j}\right)=d_{i j} \pm 2 k^{2} / n^{2}$ for every $1 \leq i \leq j \leq k$. $\diamond$

Remark 6.2 (divisibility). The factor $2 k^{2} / n^{2}$ is only required in the formal definition of $\Pi_{k, \bar{d}, \bar{\gamma}}$ so that $\Pi_{k, \bar{d}, \bar{\gamma}}$ is not empty for certain values of $n$, because of divisibility problems. Henceforth, we will ignore such divisibility problems and assume graphs in $\Pi_{k, \bar{d}, \bar{\gamma}}$ have density $\mathrm{d}\left(V_{i}, V_{j}\right)$ exactly $d_{i j}$. Likewise, we will also assume the size of every class in an equipartition $\left\{V_{i}\right\}_{i=1}^{k}$ is exactly $\frac{n}{k}$. Indeed, when considering large enough graphs, such error terms are vanishing small and, therefore, it is straightforward to absorb them into the additive constant error terms, at the cost of a decreased readability.

For the particular case in which $\gamma_{i j}=1$ for every $1 \leq i \leq j \leq k-$ i.e., for which there is no restriction on how regular any pair of cluster is — we denote the property simply by $\Pi_{k, \bar{d}}=\Pi_{k, \bar{d}, \overline{1}}$. Goldreich, Goldwasser and Ron [GGR98] proved $\Pi_{k, \bar{d}}$ is testable with sample complexity polynomial in $\varepsilon^{-1}$, but exponential in $k$. In [GGR98], the authors showed that, for many graph properties $\mathcal{P}$, the problem of testing $\mathcal{P}$ can be reduced to the problem of testing $\Pi_{k, \bar{d}}$ for an appropriated $k$ and $\bar{d}$.

In general, for an arbitrary $\bar{\gamma}$, a result from Fischer, Matsliah and Shapira [FMS10] implies $\Pi_{k, \bar{d}, \bar{\gamma}}$ is also testable with sample complexity polynomial in $\varepsilon^{-1}$ and exponential in $k$. The following theorem states the sample complexity required to test $\Pi_{k, \bar{d}, \bar{\gamma}}$ is polynomial in both $\varepsilon^{-1}$ and $k$.

Theorem 6.3. For every graph property $\Pi_{k, \bar{d}, \bar{\gamma}}$ and every $\varepsilon>0$, there is an $\varepsilon$-tester for $\Pi_{k, \bar{d}, \bar{\gamma}}$ with sample complexity at most $\operatorname{poly}\left(\varepsilon^{-1}, k, \gamma_{\min }\right)$, where $\gamma_{\min }=\min \left\{\gamma_{i j}: 1 \leq i \leq j \leq k\right\}$. More specifically, there is a decision algorithm that, when given an input graph $G$, samples the graph induced by a set of at most $q=q_{6.3}\left(\varepsilon^{-1}, k, \gamma_{\min }\right)=\operatorname{poly}\left(\varepsilon^{-1}, k, \gamma_{\min }\right)$ vertices of $G$ and satisfies the following:

(i) if $G \in \Pi_{k, \bar{d}, \bar{\gamma}}$, then $G$ is accepted with probability at least $\frac{2}{3}$; 
(ii) if $G$ is $\varepsilon$-far from $\Pi_{k, \bar{d}, \bar{\gamma}}$, then $G$ is rejected with probability at least $\frac{2}{3}$.

Proving Theorem 6.3 for a property $\Pi_{k, \bar{d}}$, i.e., with no regularity restrictions, follows directly from Theorem 5.1. In fact, it is enough to consider the canonical $\varepsilon$-tester with ACC being the set of samples that have an equipartition $\left\{Q_{i}\right\}_{i=1}^{k}$ with $\mathrm{d}\left(Q_{i}, Q_{j}\right)=d_{i j} \pm \frac{1}{2} \varepsilon$ and then apply the statement of Theorem 5.1 with error term, say, $\frac{1}{4} \varepsilon$. Then with high probability, graphs in $\Pi_{k, \bar{d}}$ are clearly accepted but, more importantly, graphs $\varepsilon$-far from $\Pi_{k, \bar{d}}$ cannot be accepted because having an equipartition $\left\{V_{i}\right\}_{i=1}^{k}$ with $\mathrm{d}\left(V_{i}, V_{j}\right)=d_{i j} \pm \frac{3}{4} \varepsilon$, immediately translates to being $\frac{3}{4} \varepsilon$-close to $\Pi_{k, \bar{d}}$. However, such translation is not that immediate when regularity comes into play.

Hence, in the next section we will show how one can turn a pair $(X, Y)$ with $\mathrm{d}(X, Y)$ close to $d_{i j}$ and $\operatorname{Reg}_{\text {sog }}(X, Y)$ close to $\gamma_{i j}$ into a pair $(X, Y)$ with $\mathrm{d}(X, Y)=d_{i j}$ and $\operatorname{Reg}_{\text {ged }}(X, Y)=\gamma_{i j}$ by making few edge modifications. From that, the proof of Theorem 6.3 is straightforward and showed for completion in the subsequent section.

\subsection{Fixing regularity with few edge modifications}

This section is devoted to proving the following lemma.

Lemma 6.4. For every $\gamma>0$ and $0<\varepsilon<\frac{1}{15}$, the following holds for every $-\frac{1}{8} \varepsilon^{4} \leq \delta \leq \frac{1}{8} \varepsilon^{4}, 0 \leq$ $\eta \leq \frac{1}{2} \varepsilon^{3} \gamma, 0 \leq d \leq 1$ and $m \geq 20 /\left(\varepsilon^{3} \gamma\right)$. If $(X, Y)$ is a $(\gamma+\eta)$-quad-regular pair with density $(d+\delta)$ and $|X|=|Y|=m$, then it is possible to turn $(X, Y)$ into a $\gamma$-quad-regular pair with density $d$ by making at most $\mathrm{\varepsilon m}^{2}$ edge modifications.

Before proving Lemma 6.4, we will need the following simple proposition, stating one can construct an arbitrarily quad-regular pair $(X, Y)$ from a random bipartite graph.

Proposition 6.5. For every $0 \leq d \leq 1$ and every integer $m>0$, there exists a $\frac{5}{m}$-quad-regular pair $(X, Y),|X|=|Y|=m$, of density $\mathrm{d}(X, Y)=\frac{1}{m^{2}}\left\lfloor d m^{2}\right\rfloor$.

Proof. We first construct a pair $H \in \mathcal{H}(X, Y)$ by setting $x y \in E(H)$ with probability $d$, independently for every $x \in X$ and $y \in Y$. From Lemma 2.5 (with $\lambda=2 m, C=1$ and $k=2 m^{2}$ ), we have

$$
\mathbb{P}\left(\left|\mathrm{e}(X, Y)-d m^{2}\right| \geq 2 m\right) \leq 2 \exp \left\{\frac{-(2 m / 1)^{2}}{2 m^{2}}\right\}=2 e^{-2} .
$$

Since each edge participates in at most $m^{2}$ homomorphic copies of $C_{4}$, Lemma 2.5 (with $\lambda=2 m^{3}$, $C=m^{2}$ and $k=2 m^{2}$ ) provides that

$$
\mathbb{P}\left(\left|\operatorname{Hom}_{C_{4}}(X, Y)-d^{4} m^{4}\right| \geq 2 m^{3}\right) \leq 2 \exp \left\{\frac{-\left(2 m^{3} / m^{2}\right)^{2}}{2 m^{2}}\right\}=2 e^{-2} .
$$

Hence, with probability at least $1-4 e^{-2}>0$, there exists a pair $(X, Y)$ with $\mathrm{e}(X, Y)=d m^{2} \pm 2 m$ and $\operatorname{Hom}_{C_{4}}(X, Y) \leq d^{4} m^{4}+2 m^{3}$. By arbitrarily adding/removing at most $2 m+1 \leq 3 m$ edges from such a pair, we get a new pair $(X, Y)$ with $\mathrm{e}(X, Y)=\left\lfloor d m^{2}\right\rfloor$ and $\operatorname{Hom}_{C_{4}}(X, Y) \leq d^{4} m^{4}+5 m^{3}$, which is a pair as required by the proposition.

Proof of Lemma 6.4. Let $H=(X, Y, E(H))$ be $(\gamma+\eta)$-quad-regular of density $(d+\delta)$ as in the statement of the lemma. 
If $d \leq \varepsilon / 3$, then one can simply remove all its $(d+\delta) m^{2}<(2 \varepsilon / 3) m^{2}$ edges and then add a set of $d m^{2} \leq(\varepsilon / 3) m^{2}$ edges as in Proposition 6.5. The resulting pair $(X, Y)$ has $\operatorname{Reg}_{\mathscr{q}_{0}}(X, Y) \leq 4 / m \leq \gamma$. The case $d \geq 1-\varepsilon / 3$ is symmetric.

Suppose $\varepsilon / 3<d<1-\varepsilon / 3$. It will be enough to show one can turn $H=(X, Y, E(H))$ into a pair $H^{\prime}=\left(X, Y, E\left(H^{\prime}\right)\right)$, with $\mathrm{d}\left(H^{\prime}\right)=d \pm 2 / m$ and $\ddot{\mathrm{t}}_{C_{4}}\left(H^{\prime}\right) \leq d^{4}+\gamma-2 / m$, by making a most $\varepsilon m^{2}-2 m$ edge changes. Indeed, after doing so, one could then get a pair satisfying the statement of the lemma by arbitrarily adding/removing up to $\left|d\left(H^{\prime}\right)-d\right| m^{2} \leq 2 m$ edges, so that we get a pair with density exactly $d$.

Let $p$ be such that $(1-\varepsilon)(d+\delta)+\varepsilon p=d$. By the hypothesis on $d$ and $\varepsilon<1 / 10$, we have $0 \leq p \leq 1$. In order to get the graph $H^{\prime}$ from the previous paragraph, we will do the following operation independently for every pair $x y \in(X, Y)$ :

(i) with probability $(1-\varepsilon)$ we put $x y \in E\left(H^{\prime}\right)$ if and only if $x y \in E(H)$ - in this case we say $(x, y)$ is a preserving pair.

(ii) with probability $\varepsilon p$, we make $x y$ an edge in $H^{\prime}$ (regardless of its adjacency in $H$ );

(iii) with probability $\varepsilon(1-p)$ we make $x y$ a non-edge in $H^{\prime}$ (regardless of its adjacency in $H$ );

Note that one could also describe $H^{\prime}$ as being an " $\varepsilon$-combination" between $H$ and a random bipartite graph $G_{X, Y, p}$. For each pair $x y \in(X, Y)$, we flip a coin that lands heads with probability (1$\varepsilon)$ — indicating the adjacency of $x y$ in $H^{\prime}$ must be the same as in $H$, i.e. $x y$ is preserving - , or tails with probability $\varepsilon$ - indicating the adjacency of $x y$ in $H^{\prime}$ must be the same as in $G_{X, Y, p}$.

The value of of $p$ was chosen in such a way that

$$
\mathbb{E}\left(\mathrm{d}\left(H^{\prime}\right)\right)=(1-\varepsilon)(d+\delta)+\varepsilon p=d .
$$

Let $x_{1}, x_{2} \in_{\mathrm{U}} X$ and $y_{1}, y_{2} \in_{\mathrm{U}} Y$ be taken independently at random. Let InJ be the event that $x_{1}, x_{2}, y_{1}, y_{2}$ are all distinct and let $\overline{\text { INJ }}$ be its complement, whose probability is $\mathbb{P}(\overline{\operatorname{INJ}})=2 / n$.

By a slight abuse of notation, denote by $C_{4}={ }_{0}^{0} 0$ the following concrete complete bipartite graph $C_{4}:=\{[2],[2],[2] \times[2]\}$. The expected homomorphic density of $C_{4}$ in $H^{\prime}$ can be written as

$$
\mathbb{E}\left(\ddot{\mathrm{t}}_{C_{4}}\left(H^{\prime}\right)\right)=\mathbb{P}\left(x_{i} y_{j} \in E\left(H^{\prime}\right): \forall i j \in E\left(C_{4}\right)\right)=\mathbb{P}\left(A_{E\left(C_{4}\right)}\right),
$$

where $A_{E}$ denotes precisely the event $\left[x_{i} y_{j} \in E\left(H^{\prime}\right): \forall i j \in E\right]$, for every set $E \subseteq E\left(C_{4}\right)$. Let $\rho=\left\{i j \in E\left(C_{4}\right): x_{i} y_{j}\right.$ is preserving $\}$ and $\bar{\rho}=E\left(C_{4}\right) \backslash \rho$. Since (regardless of InJ happening or not!)

$$
\left\{x_{i} y_{j}: i j \in E\left(C_{4}\right)\right\}=\left\{x_{i}, y_{j}: i j \in \rho\right\} \sqcup\left\{x_{i} y_{j}: i j \notin \rho\right\},
$$

we can write $\mathbb{E}\left(\ddot{\mathrm{t}}_{C_{4}}\left(H^{\prime}\right)\right)=\mathbb{P}\left(A_{\rho}\right) \cdot \mathbb{P}\left(A_{\bar{\rho}}\right)$.

By partitioning the probability space into events $[\rho=E(F)]$, one for each bipartite subgraph $F=$ ([2], [2], $E(F))$ of $C_{4}$, we obtain

$$
\mathbb{E}\left(\ddot{\mathrm{t}}_{C_{4}}\left(H^{\prime}\right)\right)=\sum_{F \leq C_{4}} \mathbb{P}\left(A_{\rho} \mid \rho=E(F)\right) \cdot \mathbb{P}\left(A_{\bar{\rho}} \mid \rho=E(F)\right) \cdot \mathbb{P}(\rho=E(F)) .
$$

Next, we give upper bounds for each of the three terms from the equation above. From the definition of preserving edges, if $i j \in \rho$, then the pair $x_{i} y_{j}$ has the same adjacency in both $E(H)$ and $E\left(H^{\prime}\right)$. 
Hence,

$$
\begin{aligned}
\mathbb{P}\left(A_{\rho} \mid \rho=E(F)\right) & =\mathbb{P}\left(x_{i} y_{j} \in E\left(H^{\prime}\right): \forall i j \in \rho \mid \rho=E(F)\right) \\
& =\mathbb{P}\left(x_{i} y_{j} \in E(H): \forall i j \in \rho \mid \rho=E(F)\right) \\
& =\mathbb{P}\left(x_{i} y_{j} \in E(H): \forall i j \in E(F)\right) \\
& =\ddot{\mathrm{t}}_{F}\left(H^{\prime}\right) .
\end{aligned}
$$

Likewise, if $i j \notin \rho$, then $x_{i} y_{j}$ has the same adjacency in $E\left(H^{\prime}\right)$ as in a random graph $G_{X, Y, p}$.

$$
\begin{aligned}
\mathbb{P}\left(A_{\bar{\rho}} \mid \rho=E(F)\right) & =\mathbb{P}\left(x_{i} y_{j} \in E\left(H^{\prime}\right): \forall i j \notin \rho \mid \rho=E(F)\right) \\
& =\mathbb{P}\left(x_{i} y_{j} \in E\left(G_{X, Y, p}\right): \forall i j \notin E(F)\right) \\
& \leq \mathbb{P}\left(x_{i} y_{j} \in E\left(G_{X, Y, p}\right): \forall i j \notin E(F) \mid \text { INJ }\right)+\mathbb{P}(\text { INJ }) \\
& =p^{4-\mathrm{e}(F)}+\frac{2}{m} .
\end{aligned}
$$

Note that we had to condition on INJ, for otherwise there could be repeated edges $x_{i} y_{j}$. This is also required for bounding the probability of $[\rho=E(F)]$.

$$
\mathbb{P}(\rho=E(F)) \leq \mathbb{P}(\rho=E(F) \mid \mathrm{INJ})+\mathbb{P}(\operatorname{INJ})=\varepsilon^{4-\mathrm{e}(F)}(1-\varepsilon)^{\mathrm{e}(F)}+\frac{2}{m} .
$$

By substituting the last three inequalities into (6.1), we get

$$
\begin{aligned}
\mathbb{E}\left(\ddot{\mathrm{t}}_{C_{4}}\left(H^{\prime}\right)\right) & =\sum_{F}\left(\varepsilon^{4-\mathrm{e}(F)}(1-\varepsilon)^{\mathrm{e}(F)}+\frac{2}{m}\right) \cdot\left(p^{4-\mathrm{e}(F)}+\frac{2}{m}\right) \cdot \ddot{\mathrm{t}}_{F}(H) \\
& \leq \frac{6}{m}+\sum_{F}(\varepsilon p)^{4-\mathrm{e}(F)}(1-\varepsilon)^{\mathrm{e}(F)} \cdot \ddot{\mathrm{t}}_{F}(H),
\end{aligned}
$$

where the last inequality is a consequence of $\sum_{F} \ddot{t}_{F}(H)=1$. By Lemma 2.14, we know that

$$
\ddot{\mathrm{t}}_{F}(H) \leq \frac{(d+\delta)^{4}+\gamma+\eta}{(d+\delta)^{4-\mathrm{e}(F)}}=(d+\delta)^{\mathrm{e}(F)}+\frac{\gamma+\eta}{(d+\delta)^{4-\mathrm{e}(F)}}
$$

for every $F$. Moreover, if e $(F)=1$ we obviously do not need the second "error" term, since $\ddot{\mathrm{t}}_{F}(H)=$ $(d+\delta)^{\mathrm{e}(F)}$. Hence,

$$
\mathbb{E}\left(\ddot{\mathrm{t}}_{C_{4}}\left(H^{\prime}\right)\right) \leq \frac{6}{m}+\sum_{F}(\varepsilon p)^{4-\mathrm{e}(F)}((1-\varepsilon)(d+\delta))^{\mathrm{e}(F)}+(\gamma+\eta) \cdot \sum_{F: \mathrm{e}(F) \neq 1}\left(\frac{\varepsilon p}{d+\delta}\right)^{4-\mathrm{e}(F)}(1-\varepsilon)^{\mathrm{e}(F)} .
$$

The first summation is precisely the binomial expansion of $(\varepsilon p+(1-\varepsilon)(d+\delta))^{4}=d^{4}$. The second summation can be upper bounded by

$$
\begin{aligned}
\left(\frac{\varepsilon p}{d+\delta}+(1-\varepsilon)\right)^{4}-4\left(\frac{\varepsilon p}{d+\delta}\right)^{3}(1-\varepsilon) & =\left(1-\frac{\delta}{d+\delta}\right)^{4}-4\left(\varepsilon-\frac{\delta}{d+\delta}\right)^{3}(1-\varepsilon) \\
& \leq\left(1+\varepsilon^{3} / 2\right)^{4}-4\left(\varepsilon-\varepsilon^{3} / 2\right)^{3}(1-\varepsilon) \\
& <1-2 \varepsilon^{3}+15 \varepsilon^{4} \leq 1-\varepsilon^{3}
\end{aligned}
$$

where the first inequality uses $\frac{1}{3} \varepsilon \leq d \leq 1-\frac{1}{3} \varepsilon$ and the last inequality follows from the hypothesis 
that $\varepsilon<\frac{1}{15}$. Therefore, we get that

$$
\mathbb{E}\left(\ddot{\mathrm{t}}_{C_{4}}\left(H^{\prime}\right)\right) \leq \frac{6}{m}+d^{4}+(\gamma+\eta)\left(1-\varepsilon^{3}\right) \leq d^{4}+\gamma-\left(\frac{\varepsilon^{3} \gamma}{2}-\frac{6}{m}\right) \leq d^{4}+\gamma-4 / m
$$

Let $\Delta$ be the number of edge changes required to turn $H$ into $H^{\prime}$. Since $\max \{d+\delta, 1-(d+\delta)\} \leq$ $1-\frac{1}{3} \varepsilon+\delta<1-\frac{1}{4} \varepsilon$, we have

$$
\mathbb{E}\left(\Delta / m^{2}\right)=\varepsilon\left((1-p)(d+\delta)+p(1-(d+\delta)) \leq \varepsilon-\frac{1}{4} \varepsilon^{2}<\varepsilon-\frac{1}{4} \varepsilon^{3} \gamma<\varepsilon-\frac{2}{m} .\right.
$$

Note that each of the three random variables $\mathrm{d}\left(H^{\prime}\right), \ddot{\mathrm{t}}_{C_{4}}\left(H^{\prime}\right)$ and $\left(\Delta / m^{2}\right)$ can be expressed as a function satisfying the statement of Lemma 2.5 where $q=m^{2}, C \leq 1 / m^{2}$ and each $Z_{i}$ corresponds to the decision made for each pair $x y \in(X, Y)$. Thus, for each of them, the probability of deviating by $\frac{2}{m}$ from its expected value is at most $2 \exp \left\{-(2 / m)^{2} /\left(2 m^{2} / m^{4}\right)\right\}=2 e^{-2}$, which implies we must have

$$
\left|\mathrm{d}\left(H^{\prime}\right)-d\right| \leq 2 / m \quad \text { and } \quad \ddot{\mathrm{t}}_{C_{4}}\left(H^{\prime}\right) \leq d^{4}+\gamma-2 / m \quad \text { and } \quad \Delta<\varepsilon m^{2}-2 m .
$$

with probability at least $1-6 e^{-2}>0$. So there must be a concrete graph $H^{\prime}=(X, Y)$ as above.

Finally, one can turn $H^{\prime}$ into a pair $(X, Y)$ satisfying the statement of the lemma by arbitrarily adding/removing up to $\left|d\left(H^{\prime}\right)-d\right| m^{2} \leq 2 m$ edges so that we end up with a pair of density exactly $d$ (see Remark 6.2) - making such changes increases $\ddot{t}_{C_{4}}\left(H^{\prime}\right)$ by at most $2 / m$, i.e., yields $\ddot{\mathrm{t}}_{C_{4}}\left(H^{\prime}\right) \leq d^{4}+\gamma$, as desired.

\subsection{Proof of Theorem 6.3}

Let $\varepsilon^{\prime}=\frac{1}{16} \varepsilon^{4} \gamma_{\min }$ and $q=q_{5.2}\left(\varepsilon^{\prime}, k, \frac{2}{3}\right)$. Consider an algorithm that, given an input graph $G=(V, E)$, first samples a set $Q \in\left(\begin{array}{c}V \\ q\end{array}\right)$ and then accepts if and only if $G[Q]$ has an equipartition $\left\{Q_{i}\right\}_{i=1}^{k}$ satisfying the following for every $1 \leq i \leq j \leq k$ :

(i) $\mathrm{d}\left(Q_{i}, Q_{j}\right)=d_{i j} \pm \varepsilon^{\prime}$;

(ii) $\left(Q_{i}, Q_{j}\right)$ is $\left(\gamma_{i j}+\varepsilon^{\prime}\right)$-quad-regular.

We will show such an algorithm is an $\varepsilon$-tester for $\Pi_{k, \bar{d}, \bar{\gamma}}$. Assume the sample $Q \in\left(\begin{array}{l}V \\ q\end{array}\right)$ satisfies the assertion of Theorem 5.2 (with error parameter $\varepsilon^{\prime}$ ), which happens with probability at least $\frac{2}{3}$, by the choice of $q$.

Suppose $G \in \Pi_{k, \bar{d}, \bar{\gamma}}$. By Theorem 5.2, the algorithm indeed accepts, since $G[Q]$ has an equipartition $\left\{Q_{i}\right\}_{i=1}^{k}$ such that $\mathrm{d}\left(Q_{i}, Q_{j}\right)=d_{i j} \pm \varepsilon^{\prime}$ and $\operatorname{Reg}_{g_{s}}\left(Q_{i}, Q_{j}\right) \leq \gamma_{i j}+\varepsilon^{\prime}$.

On the other hand, suppose $G$ is accepted by the algorithm. Then, by Theorem 5.2, $G$ must have an equipartition $\left\{V_{i}\right\}_{i=1}^{k}$ such that $\mathrm{d}\left(V_{i}, V_{j}\right)=d_{i j} \pm 2 \varepsilon^{\prime}=d_{i j} \pm \frac{1}{8} \varepsilon^{4}$ and $\operatorname{Reg}_{g_{a}}\left(V_{i}, V_{j}\right) \leq \gamma_{i j}+2 \varepsilon^{\prime} \leq$ $\gamma_{i j}+\frac{1}{2} \varepsilon^{3} \gamma$. By applying Lemma 6.4 to each $\left(V_{i}, V_{j}\right)$, we can turn $G$ into a graph satisfying the property $\Pi_{k, \bar{d}, \bar{\gamma}}$ by making at most $k^{2} \cdot \varepsilon(n / k)^{2} \leq \varepsilon n^{2}$ edge modifications. It follows that if $G$ is $\varepsilon$-far from $\Pi_{k, \bar{d}, \bar{\gamma}}$, then $G$ must be rejected by the algorithm. 


\section{Chapter 7}

\section{Estimation of parameters by quotient queries}

In this brief chapter we summarize the implications of Theorem 5.1 and Theorem 5.2 to property testing and estimation of parameters.

We recall that Theorem 5.1 states that a typical small random sample of $G$ carries the meaningful information of all its cluster graphs of order $k$, i.e., of the set $G / \wp_{k}$. It easily follows that if a graph parameter $f$ can be efficiently approximated by some function $f^{*}$ that is defined over the set $G / \wp_{k}$ - and such function $f^{*}$ is well behaved with respect to small perturbations to cluster graphs then we can give reasonable bounds for the sample complexity required to estimate $f$. The precise statement is given in Section 7.1.

Based on this implication, we then consider the study of a different type of algorithm for testing properties and estimating parameters. Recall that the algorithms considered so far in this thesis do not access the input graph $G$ directly, but by making queries to an oracle; such queries, in the so called dense-model, are of the type: "Are $u$ and $v$ adjacent in $G$ ?", where $u$ and $v$ are vertices from $G$ (which is essentially equivalent to selecting a random sample of $G$ by [GT03, Theorem 2]). In Section 7.2, we will consider algorithms that access the input graph $G$ by performing the following type of query: "does $G$ have a partition close to $R$ ?", where $R \in \mathcal{G}^{*}([k])$ is some given weighted graph.

\subsection{Parameters given by optimization problems over $G / \wp_{K}^{\leqslant}$}

The following result states that graph parameters, that can be expressed as the optimal value of some optimization problem over the set $G / \wp_{K}^{\leqslant}$of all cluster graphs of $G$ of vertex order at most $K$, can be estimated with a query complexity that is only polynomial in $K$ and in the error parameter $\varepsilon$.

Theorem 7.1. Let $z: \mathcal{G} \rightarrow \mathbb{R}$ be a graph parameter and suppose that there is a weighted graph parameter $z^{*}: \mathcal{G}^{*} \rightarrow \mathbb{R}$ and constants $K>0$ and $c>0$ such that

(i) $z(\Gamma)=\max _{R \in \Gamma / \wp_{K}^{\leq}} z^{*}(R)$ for every $\Gamma \in \mathcal{G}$, and

(ii) $\left|z^{*}(R)-z^{*}\left(R^{\prime}\right)\right| \leq c \cdot \mathbf{d}_{1}\left(R, R^{\prime}\right)$ for all weighted graphs $R, R^{\prime} \in \mathcal{G}^{*}$ on the same vertex set.

Then $z$ is estimable with sample complexity $q(\varepsilon)=q_{7.1}(\varepsilon)=\operatorname{poly}(K, c / \varepsilon)$. 
Proof of Theorem 7.1. Fix $\varepsilon>0$ and an input graph $\Gamma \in \mathcal{G}(V)$. Let $q=q_{5.1}(\varepsilon / c, K, \delta)$, where $\delta=1 /(3 K)$. Choose $Q$ uniformly from all subsets of $V$ of size $q$ and set $\bar{\Gamma}=\Gamma[Q]$. It is enough to show that $z(\Gamma)=z(\bar{\Gamma}) \pm \varepsilon$ if the assertions Theorem 5.1(i) and (ii) hold for every equipartition of $\Gamma$ and $\bar{\Gamma}$ (respectively) of size at most $K$. Indeed, the probability of that happening is, by applying Theorem 5.1 for $k=1, \ldots, K$, at least $1-\delta K=\frac{2}{3}$.

Let $\mathcal{V} \in \wp_{K}^{\leq}(V)$ be an equipartition of $\Gamma$ such that $z(\Gamma)=z^{*}(\Gamma / \mathcal{V})$. We can assume, by above, that there is an equipartition $\overline{\mathcal{V}}$ of $\bar{\Gamma}$ such that $\mathbf{d}_{1}(\Gamma / \mathcal{V}, \bar{\Gamma} / \overline{\mathcal{V}}) \leq \varepsilon / c$. By the second condition on $z^{*}$ in the statement of Theorem 7.1 , we have $\left|z^{*}(\bar{\Gamma} / \overline{\mathcal{V}})-z^{*}(\Gamma / \mathcal{V})\right| \leq \varepsilon$, and therefore $z(\bar{\Gamma}) \leq$ $z^{*}(\bar{\Gamma} / \overline{\mathcal{V}}) \leq z^{*}(\Gamma / \mathcal{V})+\varepsilon=z(\Gamma)+\varepsilon$

A symmetric argument relying on Theorem 5.1(ii) shows that $z(\Gamma) \leq z(\bar{\Gamma})+\varepsilon$.

Theorem 7.1 was proven in $\left[\mathrm{HKL}^{+} 20\right.$, Theorem 3.2], with a worse sample complexity (since we were relying on previous worse bounds for Theorem 5.1). The following easy consequence of Theorem 7.1 will be used in Chapters 8 and 9 .

Corollary 7.2. Let $z: \mathcal{G} \rightarrow \mathbb{R}$ be a graph parameter and $c \geq 1$ such that for every $\gamma>0$, there is $K=K_{7.2}(\gamma)$ and a weighted graph parameter $z^{*}: \mathcal{G}^{*}([K]) \rightarrow \mathbb{R}$ satisfying

(i) $z(\Gamma)=\max _{\mathcal{V} \in \wp_{K}} z^{*}(\Gamma / \mathcal{V}) \pm \gamma$ for every $\Gamma \in \mathcal{G}$, and

(ii) $\left|z^{*}(R)-z^{*}\left(R^{\prime}\right)\right| \leq c \mathbf{d}_{1}\left(R, R^{\prime}\right)$ for all weighted graphs $R, R^{\prime} \in \mathcal{G}^{*}([K])$.

Then $z$ is estimable with sample complexity $q_{7.1}\left(K\left(\frac{1}{3} \varepsilon\right), 3 c / \varepsilon\right)$.

Proof. For every given $\varepsilon>0$, put $\gamma=\frac{1}{3} \varepsilon$, and let $K=K_{7.2}\left(\frac{1}{3} \varepsilon\right)$. Let $z^{*}: \mathcal{G}^{*}([K]) \rightarrow \mathbb{R}$ be as in the statement of the corollary and define the parameter $f: \Gamma \mapsto \max _{\mathcal{V} \in \wp_{K}^{\leqslant}} z^{*}(\Gamma / \mathcal{V})$. Set $q$ as in the statement of Theorem 7.1 with inputs $K$ and $\gamma$. In particular, we get that if $Q \in_{\mathrm{U}}\left(\begin{array}{c}V \\ q\end{array}\right)$, then

$$
f(\Gamma)=f(\Gamma[Q]) \pm \gamma
$$

with probability at least $\frac{2}{3}$. Since, by hypothesis, we have $z(\Gamma)=f(\Gamma) \pm \gamma$ and $z(\Gamma[Q])=f(\Gamma[Q]) \pm \gamma$, if follows that

$$
z(\Gamma)=z(\Gamma[Q]) \pm \varepsilon
$$

as desired.

We use Corollary 7.2 to estimate the distance to monotone and hereditary graph parameters in the next two chapters.

We conclude this section by observing that, despite the fact that the parameters $z$ considered in Corollary 7.2 seem to have a somewhat restricted form, it is possible to show that they include all estimable parameters, with the caveat that one may have to consider very large values of $K$.

\section{2 $\quad$ Property testing by quotients}

In this section, we introduce a different notion of testability and discuss its relation with the standard one. The remainder of the thesis does not depend on this section.

We recall that, given two weighted graphs $R, R^{\prime} \in \mathcal{G}^{*}(V)$ over the same set $V$ of vertices, we write $R \leq R^{\prime}$ if $R(i, j) \leq R^{\prime}(i, j)$ for every $i, j \in V$. Moreover, for any constant $\eta \in \mathbb{R}$ we write $R^{\prime}=R+\eta$ if $R^{\prime}(i, j)=R(i, j)+\eta$ for every $i, j \in V$. 
Definition 7.3 $((K, \eta)$-quot-access). For every integer $K>0$ and every $\eta>0$, we say an algorithm $\mathcal{T}$ has $(K, \eta)$-quot-access to a graph $G=(V, E)$ if $\mathcal{T}$ has access to an oracle $\mathcal{O}$ that answers the following type of query about equipartitions of $G$ of size at most $K$. Given a query $\left(R_{\min }, R_{\max }\right) \in\left(\mathcal{G}^{*}([k]), \mathcal{G}^{*}([k])\right)$, with $k \leq K$ and $R_{\min } \leq R_{\max }$, the oracle $\mathcal{O}$ is required to output

(i) IN, if there is an equipartition $\mathcal{V}=\left\{V_{i}\right\}_{i=1}^{k}$ of $G$ satisfying

$$
R_{\min } \leq G / \mathcal{V} \leq R_{\max }
$$

(ii) OUt, if no equipartition $\mathcal{V}=\left\{V_{i}\right\}_{i=1}^{k}$ of $G$ satisfies $R_{\min }-\eta \leq G / \mathcal{V} \leq R_{\max }+\eta$.

If neither of the above conditions holds, then the oracle is allowed to output either IN or OUT, arbitrarily.

Next, given a graph property $\mathcal{P}$, we can consider algorithms that distinguishes between graphs in $\mathcal{P}$ and graphs far from $\mathcal{P}$ by only having quot-access to the input graph.

Definition 7.4 (quot-testable). A graph property $\mathcal{P}$ is quot-testable if for every $\varepsilon>0$, there exist $\eta=\eta_{7.4}(\varepsilon)>0$, an integer $K=K_{7.4}(\varepsilon)$ and a decision algorithm $\mathcal{T}$ which behaves as follows when given $(K, \eta)$-quot-access to an input graph $G$ :

(i) Accepts $G$, if $G \in \mathcal{P}$;

(ii) Rejects $G$, if $G$ is $\varepsilon$-far from $\mathcal{P}$.

An algorithm $\mathcal{T}$ as above is called an $\varepsilon$-quot-tester for $\mathcal{P}$ with complexity $(K(\varepsilon), \eta(\varepsilon))$ - note that this complexity does not depend on the actual number of oracle queries performed by $\mathcal{T}$. We say that $\mathcal{P}$ is easily-quot-testable if for every $\varepsilon$, there is one such $\mathcal{T}$ with $K(\varepsilon)$ and $\eta(\varepsilon)^{-1}$ both bounded by $\operatorname{poly}\left(\varepsilon^{-1}\right)$.

Theorem 7.5. Let $\mathcal{P}$ be a graph property quot-testable with complexity $(K(\varepsilon), \eta(\varepsilon))$. Then $\mathcal{P}$ is testable with sample complexity $q=q_{5.1}\left(K(\varepsilon), \eta(\varepsilon) / 2, \frac{2}{3}\right)$.

Proof. Let $\varepsilon>0$ be fixed and let $K=K(\varepsilon)$ and $\eta=\eta(\varepsilon)$. We are going to design a randomized algorithm $\mathcal{O}^{\prime}$ with sample complexity $q=q_{5.1}\left(K(\varepsilon), \eta(\varepsilon) / 2, \frac{2}{3}\right)$ that, with probability at least $\frac{2}{3}$, behaves as the oracle described in Definition 7.3 - that is, such that for every graph $G=(V, E)$ and every query $\left(R_{\min }, R_{\max }\right)$, the output of $\mathcal{O}^{\prime}$ satisfies items (i) and (ii) from Definition 7.3. It is not hard to see that feeding such an algorithm to an $\varepsilon$-quot-tester for $\mathcal{P}$ yields an $\varepsilon$-tester for $\mathcal{P}$ as required.

Algorithm $\mathcal{O}^{\prime}$ simply samples a set $Q \subseteq V$ of size $q$ and, given a query $\left(R_{\min }, R_{\max }\right)$ with $R_{\min } \leq R_{\max } \in \mathcal{G}^{*}([k])$ and $k \leq K$, outputs

(i) IN, if there exists an equipartition $\mathcal{Q}=\left\{Q_{i}\right\}_{i=1}^{k}$ of $Q$ satisfying $R_{\min }-\eta / 2 \leq G[Q] / \mathcal{Q} \leq$ $R_{\max }+\eta / 2$

(ii) Out, otherwise. 
Theorem 5.1 tells us that, with probability at least $\frac{2}{3}$ over the choice of $Q$, the sample $G[Q]$ is faithful, in the sense it satisfies the assertion of Theorem 5.1 with error parameter $\eta / 2$. We will show that $\mathcal{O}^{\prime}$ behaves as in Definition 7.3 in that case. Indeed, if $G$ has an equipartition $\mathcal{V}=\left\{V_{i}\right\}_{i=1}^{k}$ for which $R_{\text {min }} \leq G / \mathcal{V} \leq R_{\max }$, then since $G[Q]$ is faithful, there must be an equipartition $\mathcal{Q}$ of $Q$ that makes $\mathcal{O}^{\prime}$ answer In. On the other hand, if the algorithm $\mathcal{O}^{\prime}$ above does answer IN, again because $G[Q]$ is faithful, there must be an equipartition $\mathcal{V}=\left\{V_{i}\right\}_{i=1}^{k}$ satisfying $R_{\min }-\eta \leq G[Q] / \mathcal{Q} \leq R_{\max }+\eta$

Corollary 7.6. If a graph property $\mathcal{P}$ is easily-quot-testable, then $\mathcal{P}$ is easily-testable.

Proof. It suffices to recall that $q_{5.1}\left(\varepsilon, K, \frac{2}{3}\right)=\operatorname{poly}\left(\varepsilon^{-1}, K\right)$.

One might ask whether the converse of Corollary 7.6 holds, that is, whether every easily-testable property $\mathcal{P}$ is also easily-quot-testable. Unfortunately, we believe the answer is negative.

On the other hand, we could introduce a more general concept, namely that of easily-reg-quottestable properties by allowing regularity constraints in each oracle query. We could then prove a result analogous to Theorem 7.5 based on Theorem 5.2 instead of Theorem 5.1. We do not elaborate this further, as this would be quite similar to the material above, and it would be somewhat more technical. However, we mention this concept here because we think it would be interesting to investigate if every easily-testable property is easily-reg-quot-testable as well.

Furthermore, even though we mentioned above that we do not think the converse of Corollary 7.6 holds, giving a characterization of easily-quot-testable properties could be an interesting topic for further research. Indeed, not only is quot-testability related to standard property testing, as discussed above, but it could be valuable to understand which kinds of properties can be tested when an algorithm is given access to the projections of an object on small dimensional spaces.

Analogously, one could also study the problem of parameters estimation under the framework of algorithms which are only given quot-access to the input graph. We discuss this approach briefly in Section 9.3. 


\section{Chapter 8}

\section{Estimating the entropy of monotone graph properties}

The focus of this chapter will be monotone properties. As we have seen in Chapter 4, a monotone property $\mathcal{P}$ can be described by the class $\operatorname{Forb}(\mathcal{F})$ of graphs containing no copy (not necessarily induced) of members of $\mathcal{F}$, for a certain family $\mathcal{F}$ of graphs. Since we only consider not necessarily induced copies in this chapter, we will say that a graph $G$ is $\mathcal{F}$-free if $G \in \operatorname{Forb}(\mathcal{F})$ and that $G$ is $F$-free if $G \in \operatorname{Forb}(F)=\operatorname{Forb}(\{F\})$.

Let $\mathcal{F}$ be a certain graph family. The main purpose of this chapter is to estimate the graph parameter the number of $\mathcal{F}$-free spanning subgraphs of the input graph $\Gamma$. Recall that $G^{\prime} \in \mathcal{G}(V)=$ $\{0,1\}^{V \times V}$ is a spanning subgraph of a graph $G \in \mathcal{G}(V)$ if $G^{\prime} \leq G$, that is, if $E\left(G^{\prime}\right) \subseteq E$.

Given a graph $\Gamma \in \mathcal{G}$ and a family $\mathcal{F}$ of graphs, we denote the set of all $\mathcal{F}$-free spanning subgraphs of $\Gamma$ by $\operatorname{Forb}(\Gamma, \mathcal{F})=\{G$ is a spanning subgraph of $\Gamma: G \in \operatorname{Forb}(\mathcal{F})\}$. We will consider the parameter $h_{\mathcal{F}}$ defined below.

Definition 8.1 (entropy of $\operatorname{Forb}(\Gamma, \mathcal{F})$ ). Given a family $\mathcal{F}$ of graphs and a graph $\Gamma=(V, E)$, we define the entropy of $\operatorname{Forb}(\Gamma, \mathcal{F})$ as

$$
h_{\mathcal{F}}(\Gamma)=\frac{1}{|V(\Gamma)|^{2}} \log _{2}|\operatorname{Forb}(\Gamma, \mathcal{F})| .
$$

For example, if $\mathcal{F}=\left\{K_{3}\right\}$ and $\Gamma=K_{n}$, computing $h_{\mathcal{F}}$ requires estimating the number of $K_{3}$-free subgraphs of $K_{n}$ up to a multiplicative error of $2^{o\left(n^{2}\right)}$ :

$$
h_{\mathcal{F}}\left(K_{n}\right)=\frac{1}{n^{2}} \log _{2}|\operatorname{Forb}(\Gamma, \mathcal{F})|=\frac{1}{n^{2}} \log _{2} 2^{\frac{1}{2}\left(\begin{array}{c}
n \\
2
\end{array}\right)+o\left(n^{2}\right)} \rightarrow \frac{1}{4} .
$$

This was done by Erdős, Kleitman and Rothschild for $\mathcal{F}=\left\{K_{r}\right\}$ [EKR76], see also Erdős, Frankl and Rödl [EFR86] for $F$-free subgraphs. Counting problems of this type were studied by several people. Consider for instance, the work of Prömel and Steger [PS92, PS96], the logarithmic density in Bollobás [Bol98], and some more recent results about the number of $n$-vertex graphs avoiding copies of some fixed forbidden graphs [BBS04, BS11]. Algorithmic aspects have been investigated by Duke, Lefmann and Rödl [DLR95] and, quite recently, by Fox, Lovász and Zhao [FLZ17].

As we shall see, estimating graph parameters $h_{\mathcal{F}}(\Gamma)$ is related to estimating distances of 
graphs from the corresponding graph property $\mathcal{P}=\operatorname{Forb}(\mathcal{F})$. Improving upon the approach of Fischer and Newman [FN07], Alon, Shapira and Sudakov [ASS09, Theorem 1.2] proved that the distance to every monotone graph property $\mathcal{P}$ is estimable using a natural algorithm, which simply computes the distance from the induced sampled graph to $\mathcal{P}$. However, one disadvantage of this approach is that the accuracy of the estimate relies heavily on stronger versions of Szemerédi's Regularity Lemma [AFKS00, Sze78]. Therefore, the query complexity given by such approach is at least of the order TOWER(poly $(1 / \varepsilon))$ Moreover, it follows from a result of Gowers [Gow97] that any approach based on Szemerédi's Regularity Lemma cannot lead to a bound that is better than $\operatorname{TOWER}(\operatorname{poly}(1 / \varepsilon))$.

In this chapter, we introduce the concept of recoverable graph properties. Roughly speaking, given a function $f:(0,1] \rightarrow \mathbb{R}$, we say that a graph property $\mathcal{P}$ is " $f$-recoverable" if every large graph $G \in \mathcal{P}$ is $\varepsilon$-close to admitting a partition $\mathcal{V}$ of its vertex set into at most $f(\varepsilon)$ classes that witnesses membership in $\mathcal{P}$, i.e., such that any graph that can be partitioned in the same way must be in $\mathcal{P}$. The precise definition of an $f$-recoverable graph property is given in Definition 8.6.

Let $\mathcal{F}$ be a family of graphs for which $\operatorname{Forb}(\mathcal{F})$ is $f$-recoverable, for some function $f:(0,1] \rightarrow \mathbb{R}$. The first main result of this chapter states that $h_{\mathcal{F}}$ can be estimated with a sample complexity that does not grow much faster than $f$.

Theorem 8.2. Let $\operatorname{Forb}(\mathcal{F})$ be an $f$-recoverable graph property, for some function $f:(0,1] \rightarrow \mathbb{R}$. Then, for all $\varepsilon>0$ there is $n_{0}$ such that, for any graph $\Gamma$ with $|V(\Gamma)| \geq n_{0}$, the graph parameter $h_{\mathcal{F}}$ of Definition 8.1 can be estimated within an additive error of $\varepsilon$ with sample complexity $\operatorname{poly}(f(\varepsilon / 6) / \varepsilon)$.

One could apply strong versions of regularity to show that every monotone property $\operatorname{Forb}(\mathcal{F})$ is $f$ recoverable. Such approach would, however, provide an upper bound of at least $\operatorname{TOWER}\left(\operatorname{poly}\left(\varepsilon^{-1}\right)\right)$ for the function $f$. We find a connection between this notion of recoverability and the graph Removal Lemma (Lemma 3.27), which can lead to better bounds for the function $f(\varepsilon)$.

We show that every monotone graph property $\operatorname{Forb}(\mathcal{F})$ is $f$-recoverable for some function $f$ that is only exponential in the bounds given by the Removal Lemma for the family $\mathcal{F}$. For the proof, we use a weighted version of the Removal Lemma (see Lemma 8.7).

Theorem 8.3. For every family $\mathcal{F}$ of graphs, the property $\operatorname{Forb}(\mathcal{F})$ is $f$-recoverable for $f(\varepsilon)=$ $n_{0} 2^{\mathrm{poly}(M / \delta)}$, where $\delta, M$ and $n_{0}$ are as in Lemma 8.7 with input $\mathcal{F}$ and $\varepsilon$.

The case of $\mathcal{F}$ finite is an instance where the bounds given by Lemma 8.7 relate polynomially with the bounds of Lemma 3.27. In particular, Theorem 8.3, together with the bounds for Lemma 3.27 obtained by Fox [Fox11] for finite families $\mathcal{F}$, implies that $\operatorname{Forb}(\mathcal{F})$ is $f$-recoverable with $f(\varepsilon)=$ TOWER $(\operatorname{poly}(\log (1 / \varepsilon)))$. Naturally, one can get much better bounds for families $\mathcal{F}$ admitting an efficient removal lemma, i.e., families $\mathcal{F}$ for which $\operatorname{Forb}(\mathcal{F})$ can be efficiently tested see Sections 3.6.3 and 4.3.2 for a brief review of the current literature regarding such families.

The remainder of the chapter is structured as follows. In Section 8.1, we introduce the concept of recoverable graph properties and prove Theorem 8.3. Theorem 8.2 is a consequence of Theorem 8.9, which is the main result in Section 8.2. In Section 8.3 we establish the relation between $h_{\mathcal{F}}(\Gamma)$ with the distance $\mathbf{d}_{1}(\Gamma, \operatorname{Forb}(\mathcal{F}))$ from the property $\operatorname{Forb}(\mathcal{F})$. We finish the chapter with some concluding remarks in Section 8.4. 


\subsection{Recoverable graph properties}

The main objective of this section is to introduce the concept of $\varepsilon$-recoverability and to state our main results in terms of it. That will allow us to express (see Section 8.2) the parameter $h_{\mathcal{F}}$ introduced in Definition 8.1, in terms of the solution of a suitable optimization problem over the set $\Gamma / \wp_{K}^{\leqslant}$of cluster graphs of $\Gamma$ of vertex size at most $K$. By doing so, we get our bounds for estimating $h_{\mathcal{F}}$ as a consequence of Theorem 7.1.

\subsubsection{Recovering partitions}

We are interested in the property of graphs that are free of copies of members of a (possibly infinite) family $\mathcal{F}$ of graphs. Since weighted graphs will represent cluster graphs associated with a partition of the vertex set of the input graph, it will be convenient to work with the following property of weighted graphs:

$$
\operatorname{Forb}_{>}(\mathcal{F})=\left\{R \in \mathcal{G}^{*}: \mathrm{t}(F ; R)=0 \text { for every } F \in \mathcal{F}\right\} .
$$

Let $R, S \in \mathcal{G}^{*}(V)$ be weighted graphs on the same vertex set $V$. We say that $S$ is a spanning subgraph of $R$, which will be denoted by $S \leq R$, if $S(i, j) \leq R(i, j)$ for every $(i, j) \in V \times V$. When there is no ambiguity, we will just say that $S$ is a subgraph of $R$. We also define

$$
\operatorname{Forb}_{\rightarrow}(R, \mathcal{F})=\left\{S \in \operatorname{Forb}_{>}(\mathcal{F}): S \leq R\right\}
$$

The following result shows that having a cluster graph in Forb $_{>}(\mathcal{F})$ witnesses membership in $\operatorname{Forb}(\mathcal{F})$.

Proposition 8.4. Let $\mathcal{F}$ be a family of graphs and let $\mathcal{V}$ be an equipartition of a graph $G$. If $G / \mathcal{V} \in$ $\operatorname{Forb}_{>}(\mathcal{F})$, then $G \in \operatorname{Forb}(\mathcal{F})$.

Proof. Let $\mathcal{V}=\left\{V_{i}\right\}_{i=1}^{k}$ be an equipartition of $G$ and let $R=G / \mathcal{V}$. Fix an arbitrary element $F \in \mathcal{F}$ and an arbitrary injective mapping $\varphi: V(F) \hookrightarrow V(G)$. Define the function $\psi: V(F) \rightarrow V(R)$ by $\psi(v)=i$ if $\varphi(v) \in V_{i}$. Now, if $\mathrm{t}(F ; R)=0$, there must be some edge $(u, w) \in E(F)$ such that $R(\psi(u), \psi(w))=0$, thus $G(\varphi(u), \varphi(v))=0$ and hence $\operatorname{hw}_{\varphi}(F ; G)=0$. Since $\varphi$ and $F$ were taken arbitrarily, we must have $G \in \operatorname{Forb}(\mathcal{F})$.

It is easy to see that the converse of Proposition 8.4 does not hold in general. Indeed, there exist graph families $\mathcal{F}$ and graphs $G \in \operatorname{Forb}(\mathcal{F})$ such that $G / \mathcal{V}$ is actually very far from being in $\operatorname{Forb}_{\rightarrow}(\mathcal{F})$ for some equipartition $\mathcal{V}$ of $G$. As an example, let $G$ be the $n$-vertex bipartite Turán graph $T_{2}(n)$ for the triangle $K_{3}$ with partition $V(G)=A \cup B$ and consider $\mathcal{V}=\left\{V_{i}\right\}_{i=1}^{t}$ with $V_{i}=A_{i} \cup B_{i}, i=1, \ldots, t$, where $\left\{A_{i}\right\}_{i=1}^{t}$ and $\left\{B_{i}\right\}_{i=1}^{t}$ are equipartitions of $A$ and $B$ respectively. Then $G / \mathcal{V}$ has weight $1 / 2$ on every edge, so that the distance of $G / \mathcal{V}$ to the family $\operatorname{Forb}_{>}\left(\left\{K_{3}\right\}\right)$ tends to $1 / 4$ for $t$ large by Turán's Theorem. More generally, if $\mathcal{V}$ is a random equipartition of a triangle-free graph $G \in \operatorname{Forb}\left(\left\{K_{3}\right\}\right)$ with large edge density, then with high probability the cluster graph $G / \mathcal{V}$ is still approximately $1 / 4$-far from being in Forb $_{>}\left(\left\{K_{3}\right\}\right)$.

On the other hand, we will prove that there exist partitions for graphs in $\operatorname{Forb}(\mathcal{F})$ with respect to which an approximate version of the converse of Proposition 8.4 does hold, that is, we will prove that every graph in $\operatorname{Forb}(\mathcal{F})$ is not too far from having a partition into a constant number of classes 
that witnesses membership in $\operatorname{Forb}(\mathcal{F})$. We say that such a partition is recovering with respect to $\operatorname{Forb}(\mathcal{F})$. Let us make this more precise.

\subsubsection{Recoverable properties}

Definition 8.5. Let $\mathcal{P}=\operatorname{Forb}(\mathcal{F})$ be a monotone graph property. An equipartition $\mathcal{V}$ of a graph $G \in \mathcal{P}$ is $\varepsilon$-recovering for $\mathcal{P}$ if

$$
\mathbf{d}_{1}\left(G / \mathcal{V}, \operatorname{Forb}_{\rightarrow}(\mathcal{F})\right) \leq \varepsilon
$$

Definition 8.6. Let $\mathcal{P}$ be a graph property. For a fixed function $f:(0,1] \rightarrow \mathbb{R}$, we say that the class $\mathcal{P}$ is $f$-recoverable if, for every $\varepsilon>0$, there exists $n_{0}=n_{0}(\varepsilon)$ such that the following holds. For every graph $G \in \mathcal{P}$ on $n \geq n_{0}$ vertices, there is an equipartition $\mathcal{V}$ of $G$ into at most $f(\varepsilon)$ classes which is $\varepsilon$-recovering for $\mathcal{P}$.

As a simple example, one can verify that the graph property $\mathcal{P}=\operatorname{Forb}(\mathcal{F})$ of being $r$-colorable is $f$ recoverable for $f(\varepsilon)=r / \varepsilon$; here and in what follows, for simplicity, we ignore divisibility conditions and drop floor and ceiling signs. Let $G$ be a graph in $\mathcal{P}$, with color classes $C_{1}, \ldots, C_{r}$. Let $k=r / \varepsilon$. Start by fixing parts $V_{1}, \ldots, V_{t}$ of size $n / k$ each, with each $V_{i}$ contained in some $C_{j}(j=j(i))$, and leaving out fewer than $n / k$ vertices from each $C_{j}, 1 \leq j \leq r$. The sets $V_{i}, 1 \leq i \leq t$, cover a subset $C_{j}^{\prime}$ of $C_{j}$ and $X_{j}=C_{j} \backslash C_{j}^{\prime}$ is left over. We then complete the partition by taking arbitrary parts $U_{1}, \ldots, U_{k-t}$ of size $n / k$ each, forming a partition of $\bigcup_{1 \leq j \leq r} X_{j}$. The cluster graph $G / \mathcal{V}$ can be made $r$-partite by giving weight zero to every edge incident to vertices corresponding to $U_{1}, \ldots, U_{k-t}$. Therefore $G / \mathcal{V}$ is at distance at most $r / k \leq \varepsilon$ from being $r$-partite. Thus, $\mathbf{d}_{1}\left(G / \mathcal{V}, \operatorname{Forb}_{\text {hom }}^{*}(\mathcal{F})\right) \leq \varepsilon$ as required.

We finish this section by noting that the definition of $f$-recoverable properties has some similarity with the notion of regular-reducible properties $\mathcal{P}$ defined by Alon, Fischer, Newman and Shapira [AFNS09]. When dealing with monotone properties $\mathcal{P}=\operatorname{Forb}(\mathcal{F})$, the main difference is that the notion of being regular-reducible requires that every graph $G \in \mathcal{P}$ should have a regular partition such that $G / \mathcal{V}$ is close to some property $\mathcal{H}^{*}$ of weighted graphs, while the definition of $f$-recoverable properties requires only that every $G$ has a partition $\mathcal{V}$ (regular or not) such that $G / \mathcal{V}$ is close to Forb $_{\rightarrow}(\mathcal{F})$. Another difference is that $\mathcal{H}^{*}$ must be such that having a (regular) cluster graph in $\mathcal{H}^{*}$ witnesses only closeness to $\mathcal{P}$, while having a (regular or not) cluster graph in $\operatorname{Forb}_{\rightarrow}(\mathcal{F})$ witnesses membership in $\mathcal{P}$.

\subsubsection{Monotone graph properties are recoverable}

Szemerédi's Regularity Lemma [Sze78] can be used to show that every monotone (and actually every hereditary) graph property is $f$-recoverable, for $f(\varepsilon)=\operatorname{TOWER}(\operatorname{poly}(1 / \varepsilon))$. In the remainder of this section, we prove that monotone properties $\mathcal{P}=\operatorname{Forb}(\mathcal{F})$ are recoverable using a weaker version of regularity along with the Removal Lemma, which leads to an improvement on the growth of $f$ for families $\mathcal{F}$ where the Removal Lemma is known to hold with better bounds than the Regularity Lemma. 
We first derive a version of the Removal Lemma stated in the introduction (Lemma 3.27) that applies to weighted graphs and homomorphic copies.

Lemma 8.7. For every $\varepsilon>0$ and every (possibly infinite) family $\mathcal{F}$ of graphs, there exist $\delta=$ $\delta(\varepsilon, \mathcal{F}), M=M(\varepsilon, \mathcal{F})$ and $n_{0}=n_{0}(\varepsilon, \mathcal{F})$ such that the following holds. If a weighted graph $R$ such that $|V(R)|>n_{0}$ satisfies $\mathbf{d}_{1}\left(R\right.$, Forb $\left._{>}(\mathcal{F})\right) \geq \varepsilon$, then there is a graph $F \in \mathcal{F}$ with $|V(F)| \leq M$ such that $t(F, R) \geq \delta$.

To prove Lemma 8.7, we use the following auxiliary result, which follows from work of Erdős and Simonovits [ES83]. For completeness we include its proof.

Proposition 8.8. Let $\widehat{F}$ and $F$ be graphs in $\mathcal{G}$ and suppose there is a surjective homomorphism $\zeta: V(F) \rightarrow V(\widehat{F})$. Then, for every graph $H$ such that $\mathrm{t}(\widehat{F} ; H) \geq \widehat{\delta}$, we must have $\mathrm{t}(F ; H) \geq \widehat{\delta}^{\ell}$, where $\ell=(|V(F)|+1)^{|V(\widehat{F})|}$.

Proof. We will consider the particular case in which $F$ is obtained from blowing up a single vertex $v$ of $\widehat{F}$ into $r$ distinct vertices $v_{1}, \ldots, v_{r}$ with the same adjacency as $v$, hence we assume that $\zeta\left(v_{j}\right)=v$ for every $j=1, \ldots, r$ and $\zeta(u)=u$ for every $u \notin\left\{v_{1}, \ldots, v_{r}\right\}$.

Let $n=|V(H)|, \widehat{a}=|V(\widehat{F})|, a=|V(F)|=\widehat{a}+r-1$ and $\widehat{F}_{-}=\widehat{F}-v$ be the graph on $\widehat{a}-1$ vertices obtained from $\widehat{F}$ by deleting $v$. Let $N=\mathrm{t}\left(\widehat{F}_{-} ; H\right) n^{\widehat{a}-1}$ be the number of homomorphisms from $\widehat{F}_{-}$to $H$ and $\varphi_{1}, \ldots, \varphi_{N} \in V(H)^{V\left(\widehat{F}_{-}\right)}$be an enumeration of such homomorphisms. Note that $N \leq n^{\widehat{a}-1}$ and $N \geq \mathrm{t}(\widehat{F} ; H) / n \geq \widehat{\delta} n^{\widehat{a}-1}$.

For every $i \in[N]$ and $u \in V(H)$, we consider the function $\varphi_{i}^{u}$ that extends $\varphi_{i}$ by mapping $v$ to $u$. Define $Z_{i}=\left\{u \in V(H): \operatorname{hw}_{\varphi_{\mathrm{i}}^{\mathrm{u}}}(\widehat{F} ; H)=1\right\}$ and $z_{i}=\left|Z_{i}\right|$. We claim there are $z_{i}^{r}$ ways of extending $\varphi_{i}$ to a homomorphism from $F$ to $H$. Indeed, every possible extension $\varphi_{i}^{\prime}: V(F) \rightarrow V(H)$ of $\varphi_{i}$, such that $\varphi_{i}^{\prime}\left(v_{j}\right) \in Z_{i}$, for every $j=1, \ldots, r$, satisfies $\operatorname{hw}_{\varphi_{\prime}^{\prime}}(F ; H)=1$. Therefore we have $\mathrm{t}(F ; H) n^{a} \geq \sum_{i=1}^{N} z_{i}^{r}$. Since $g(x)=x^{r}$ is a convex function for $x \geq 0$ and $r \geq 1$, we get (Lemma 2.1)

$$
\mathrm{t}(F ; H) n^{a} \geq N\left(\frac{\sum_{i=1}^{N} z_{i}}{N}\right)^{r} .
$$

Now we use the fact that $\sum_{i=1}^{N} z_{i}=\mathrm{t}(\widehat{F} ; H) n^{\widehat{a}} \geq \widehat{\delta} n^{\widehat{a}}$ and our previous bounds on $N$ to obtain that

$$
\mathrm{t}(F ; H) n^{a} \geq \widehat{\delta} n^{\widehat{a}-1}\left(\frac{\widehat{\delta} n^{\widehat{a}}}{n^{\widehat{a}-1}}\right)^{r}=\widehat{\delta}^{r+1} n^{\widehat{a}+r-1}=\widehat{\delta}^{r+1} n^{a} .
$$

Therefore, $\mathrm{t}(F ; H) \geq \widehat{\delta}^{r+1} \geq \widehat{\delta}^{a+1}$. The general case may be easily obtained by induction on the number of vertices of $\widehat{F}$.

Proof of Lemma 8.7. Denote by $\widehat{\mathcal{F}}$ the set of all homomorphic images of members of $\mathcal{F}$, that is, the set of all graphs $\widehat{F} \in \mathcal{G}$ such that there is a surjective homomorphism $F \rightarrow \widehat{F}$, for some $F \in \mathcal{F}$. Let $\widehat{M}, \widehat{\delta}$ and $\widehat{n}_{0}$ be as in Lemma 3.27 with input $\widehat{\mathcal{F}}$ and $\varepsilon / 2$. We take

$$
M=\max _{\substack{\widehat{F} \in \widehat{\mathcal{F}}: \\|V(\widehat{F})| \leq \widehat{M}}} \min _{\substack{F \in \mathcal{F} \\ F \rightarrow \widehat{F}}}|V(F)|,
$$

$n_{0}=\widehat{n}_{0}, \delta=(\varepsilon / 2)^{M^{2}} \widehat{\delta}^{\ell}$, where $\ell=(M+1)^{\widehat{M}}$. 
Let $R$ be a weighted graph such that $|V(R)|>n_{0}$ and $\mathbf{d}_{1}\left(R, \operatorname{Forb}_{>}(\mathcal{F})\right) \geq \varepsilon$. We first define a graph $H \in \mathcal{G}(V(R))$ such that $H(i, j)=1$ if and only if $R(i, j) \geq \varepsilon / 2$. It follows from $\mathbf{d}_{1}\left(R, \operatorname{Forb}_{\gg}(\mathcal{F})\right) \geq \varepsilon$ that $\mathbf{d}_{1}(H, \operatorname{Forb}(\widehat{\mathcal{F}})) \geq \varepsilon / 2$. Indeed, suppose to the contrary that there exists

$$
\begin{aligned}
\mathbf{d}_{1}\left(R, R^{\prime}\right) & =\frac{1}{|V(R)|^{2}} \sum_{\substack{i \in V(R), j \in V(R)}}\left|R(i, j)-R^{\prime}(i, j)\right| \\
& =\frac{1}{|V(R)|^{2}}\left(\sum_{\substack{(i, j): \\
H(i, j)=1, H^{\prime}(i, j)=0}}\left|R(i, j)-R^{\prime}(i, j)\right|+\sum_{\substack{(i, j): \\
H(i, j)=0, H^{\prime}(i, j)=0}}\left|R(i, j)-R^{\prime}(i, j)\right|\right) \\
& \leq \frac{1}{|V(H)|^{2}} \sum_{\substack{i \in V(H) \\
j \in V(H)}}\left|H(i, j)-H^{\prime}(i, j)\right|+\frac{1}{|V(R)|^{2}} \sum_{\substack{i \in V(R) \\
j \in V(R)}} \frac{\varepsilon}{2} \\
& =\mathbf{d}_{1}\left(H, H^{\prime}\right)+\frac{\varepsilon}{2}<\varepsilon .
\end{aligned}
$$

By Lemma 3.27 there must be $\widehat{F} \in \widehat{\mathcal{F}}$, with $|V(\widehat{F})| \leq \widehat{M}$, such that $\mathrm{t}(\widehat{F} ; H) \geq \widehat{\delta}$. By definition of $M$, there must be $F \in \mathcal{F}$ such that $|V(F)| \leq M$ and there is a surjective homomorphism $F \rightarrow \widehat{F}$. It follows from Proposition 8.8 that $\mathrm{t}(F ; H) \geq \hat{\delta}^{\ell}$. Since

$$
\operatorname{hw}_{\varphi}(F ; R) \geq(\varepsilon / 2)^{|E(F)|} \operatorname{hw}_{\varphi}(F ; H) \geq(\varepsilon / 2)^{M^{2}} \operatorname{hw}_{\varphi}(F ; H)
$$

for each $\varphi: V(F) \rightarrow V(R)$, we must have

$$
\mathrm{t}(F ; R)=\frac{\sum_{\varphi} \mathrm{hw}_{\varphi}(F ; R)}{|V(R)|^{|V(F)|}} \geq\left(\frac{\varepsilon}{2}\right)^{M^{2}} \cdot \frac{\sum_{\varphi} \mathrm{hw}_{\varphi}(F ; H)}{|V(H)|^{|V(F)|}} \geq\left(\frac{\varepsilon}{2}\right)^{M^{2}} \cdot \widehat{\delta}^{\ell}=\delta .
$$

We are now ready to prove Theorem 8.3 , which establishes that every monotone graph property is $f$-recoverable.

Proof of Theorem 8.3. Let $\delta, M$ and $n_{0}$ be as in Lemma 8.7 with input $\mathcal{F}$ and $\varepsilon$ and let $\gamma=\delta /\left(8 M^{2}\right)$. We may assume that $n_{0} \geq 2 M^{2} / \delta$ without loss of generality. It suffices to show that any $\gamma$-FK-regular equipartition $\mathcal{V}=\left\{V_{i}\right\}_{i=1}^{k}$ of a graph $G \in \operatorname{Forb}(\mathcal{F})$ into $k \geq n_{0}$ classes is $\varepsilon$-recovering - indeed, Lemma 3.15 guarantees every $G$ has one such partition.

Let $R=G / \mathcal{V}$ and suppose for contradiction that $\mathbf{d}_{1}\left(R, \operatorname{Forb}_{>}(\mathcal{F})\right) \geq \varepsilon$. Then, by Lemma 8.7, we have $\mathrm{t}(F ; R) \geq \delta$ for some graph $F \in \mathcal{F}$ such that $|F| \leq M$. On the other hand, Lemma 3.23 implies

$$
\mathrm{t}(F ; G) \geq \mathrm{t}(F ; R)-4 \gamma M^{2} \geq \delta-\frac{1}{2} \delta=\frac{1}{2} \delta .
$$

Finally, note that we assumed that $G \in \operatorname{Forb}(\mathcal{F}) \subseteq \operatorname{Forb}(F)$, which implies that $\mathrm{t}(F ; G)$ should be small since, in this case, only non-injective homomorphisms contribute to $\operatorname{t}(F ; G)$. Therefore,

$$
\mathrm{t}(F ; G) \leq \frac{1}{n^{|V(F)|}}\left(\begin{array}{c}
|V(F)| \\
2
\end{array}\right) n^{|V(F)|-1} \leq \frac{|V(F)|^{2}}{n} \leq \frac{M^{2}}{n_{0}}<\frac{1}{2} \delta,
$$


which contradicts Eq. (8.2). Hence, we must have $\mathbf{d}_{1}\left(G / \mathcal{V}, \operatorname{Forb}_{>}(\mathcal{F})\right)<\varepsilon$, that is, $\mathcal{V}$ is indeed an $\varepsilon$-recovering partition of $G$.

\subsection{Estimation of $|\operatorname{Forb}(\Gamma, \mathcal{F})|$}

The objective of this section is to prove Theorem 8.2. To do this, we shall approximate the parameter $h_{\mathcal{F}}$ by the solution of an optimization problem as in Theorem 7.1. Recall that $\operatorname{Forb}_{\rightarrow}(R, \mathcal{F})=$ $\{S \leq R: \mathrm{t}(F ; S)=0$ for every $F \in \mathcal{F}\}$, and set

$$
\operatorname{ex}^{*}(R, \mathcal{F})=\frac{1}{|V(R)|^{2}} \max _{S \in \operatorname{Forb}_{>}(R, \mathcal{F})} \mathrm{e}(S),
$$

which measures the largest edge density of a subgraph of $R$ not containing a copy of any $F \in \mathcal{F}$, up to a multiplicative constant.

We shall derive Theorem 8.2 from the following auxiliary result.

Theorem 8.9. Let $\mathcal{F}$ be a family of graphs such that $\operatorname{Forb}(\mathcal{F})$ is $f$-recoverable for some function $f:(0,1] \rightarrow \mathbb{R}$. Then, for any $\varepsilon>0$, there exists $K=f(\operatorname{poly}(\varepsilon))$ and $N=\operatorname{poly}(K, \varepsilon)$ such that any graph $\Gamma$ of vertex size $n \geq N$ satisfies

$$
\frac{\log _{2}|\operatorname{Forb}(\Gamma, \mathcal{F})|}{n^{2}}=\max _{R \in \Gamma / \wp_{K}^{\leq}} \operatorname{ex}^{*}(R, \mathcal{F}) \pm \varepsilon
$$

We define the following subsets of edges of a weighted graph $R$ :

$$
\begin{aligned}
& E_{0}(R)=\{(i, j) \in V(R) \times V(R): R(i, j)=0\} \\
& E_{1}(R)=\{(i, j) \in V(R) \times V(R): R(i, j)>0\} .
\end{aligned}
$$

We will also make use of the binary entropy function, defined by $H(x)=-x \log _{2}(x)-(1-$ $x) \log _{2}(1-x)$ for $0<x<1$. Note that $H(x) \leq-2 x \log _{2} x$ for $x \leq 1 / 8$. This function has the property (cf. [Juk01, Corollary 22.2]) that the following inequality holds for $\varepsilon=k / n<1 / 2$ :

$$
\sum_{i=0}^{k}\left(\begin{array}{c}
n \\
i
\end{array}\right) \leq 2^{H(\varepsilon) n} .
$$

Proof of Theorem 8.9. Let $\mathcal{F}$ be a family of graphs such that $\operatorname{Forb}(\mathcal{F})$ is $f$-recoverable, and fix $\varepsilon>0$, without loss of generality $\varepsilon<1$. Let $\varepsilon^{\prime}=(\varepsilon / 18)^{2}$. Using that $\log _{2} \frac{1}{x^{2}} \leq \frac{1}{x}$ for $0<x \leq \frac{1}{4}$, and $H(y) \leq-2 y \log _{2} y$ for $0<y \leq \frac{1}{8}$, we infer

$$
H\left(\varepsilon^{\prime}\right)+\varepsilon^{\prime} \leq-2(\varepsilon / 18)^{2} \log _{2}(\varepsilon / 18)^{2}+(\varepsilon / 18)^{2} \leq 2(\varepsilon / 18)+(\varepsilon / 18)^{2} \leq \varepsilon / 6 .
$$

We set $K=f\left(\varepsilon^{\prime 2}\right)$ and $N \geq 2 K^{2} / \varepsilon$ big enough so that $\log _{2} N / N<\varepsilon / 3$.

Let $\Gamma$ be an $n$-vertex graph, $n \geq N$. We first show that

$$
\frac{\log _{2}|\operatorname{Forb}(\Gamma, \mathcal{F})|}{n^{2}} \geq \max _{R \in \Gamma / \wp_{K}^{\leq}} \operatorname{ex}^{*}(R, \mathcal{F})-\varepsilon
$$

Let $R=\Gamma / \mathcal{V}$ be an arbitrary cluster graph in $\Gamma / \wp_{K}^{\leqslant}$with $\mathcal{V}=\left\{V_{i}\right\}_{i=1}^{k}$ for $k \leq K$. Choose $S \in$ 
$\operatorname{Forb}_{\rightarrow}(R, \mathcal{F})$ such that $\mathrm{e}(S)=k^{2} \mathrm{ex}^{*}(R, \mathcal{F})$. Further let $G \leq \Gamma$ be the subgraph of $\Gamma$ such that $G(r, s)=0$ if there is a pair $(i, j) \in E_{0}(S)$ such that $r \in V_{i}$ and $s \in V_{j}$ and $G(r, s)=\Gamma(r, s)$ otherwise. Thus, we obtain $G$ by deleting all edges from $\Gamma$ between $V_{i}$ and $V_{j}$ if $(i, j) \in E_{0}(S)$.

Since e $(S)$ maximizes $\operatorname{ex}^{*}(R, \mathcal{F})$ it follows that $G / \mathcal{V}=S$, which implies, by Proposition 8.4, that $G \in \operatorname{Forb}(\Gamma, \mathcal{F})$. Since every subgraph of $G$ also lies in $\operatorname{Forb}(\Gamma, \mathcal{F})$, we obtain

$$
\begin{aligned}
\log _{2}|\operatorname{Forb}(\Gamma, \mathcal{F})| \geq|\mathrm{e}(G)| & =\frac{1}{2} \sum_{(i, j) \in[k] \times[k]} S(i, j)\left|V_{i}\right|\left|V_{j}\right| \geq \frac{(n-k)^{2}}{k^{2}} \mathrm{e}(S) \\
& \geq \operatorname{ex}^{*}(R, \mathcal{F}) n^{2}-k n \geq\left(\operatorname{ex}^{*}(R, \mathcal{F})-\varepsilon\right) n^{2} .
\end{aligned}
$$

Note that we used the facts that $\mathrm{e}(S) \leq k^{2} / 2$ and $n>k / \varepsilon$, as well as $\left|V_{i}\right| \geq n / k-1$ for all $i$.

Now let us prove the other direction

$$
\frac{\log _{2}|\operatorname{Forb}(\Gamma, \mathcal{F})|}{n^{2}} \leq \max _{R \in \Gamma / \wp_{K}^{\leq}} \operatorname{ex}^{*}(R, \mathcal{F})+\varepsilon
$$

We first define $\mathcal{U}=\bigcup_{G \in \operatorname{Forb}(\Gamma, \mathcal{F})} G / \wp_{K}^{\leqslant}$to be the set of all possible cluster graphs of vertex size at most $K$ of graphs in $\operatorname{Forb}(\Gamma, \mathcal{F})$. Since $\operatorname{Forb}(\mathcal{F})$ is $f$-recoverable we can define a function

$$
\begin{aligned}
\eta: \operatorname{Forb}(\Gamma, \mathcal{F}) & \rightarrow \wp_{K}^{\leqslant} \times \mathcal{U} \\
G & \mapsto(\mathcal{V}, T)
\end{aligned}
$$

where $\mathcal{V}$ is an $\left(\varepsilon^{\prime 2}\right)$-recovering partition of $G$ into $k \leq K$ classes and $T=G / \mathcal{V}$. Clearly

$$
|\operatorname{Forb}(\Gamma, \mathcal{F})| \leq\left|\wp_{K}^{\leqslant} \times \mathcal{U}\right| \cdot \max _{(\mathcal{V}, T)}\left|\eta^{-1}(\mathcal{V}, T)\right|
$$

Since each mapping from $V(\Gamma) \rightarrow[K]$ gives a partition of $V(\Gamma)$ into at most $K$ classes, we have $\left|\wp_{K}^{\leq}\right| \leq K^{n} \leq n^{n}$. Moreover, given an arbitrary graph $G \in \mathcal{G}(V)$ and any partition $\mathcal{V}$ of $V$, an edge $G / \mathcal{V}(i, j)$ may assume $n^{2}$ different values. Hence, we have $|\mathcal{U}| \leq n^{2 K^{2}} \leq n^{n}$.

Finally we make the following claim, whose proof is deferred for a moment:

$$
\log _{2}\left(\max _{(\mathcal{V}, T)}\left|\eta^{-1}(\mathcal{V}, T)\right|\right) \leq\left(\max _{R \in \Gamma / \wp_{K}^{\leq}} \operatorname{ex}^{*}(R, \mathcal{F})+\frac{\varepsilon}{3}\right) n^{2}
$$

Combining this we can take the logarithm of (8.5) to get as desired:

$$
\begin{aligned}
\log _{2}|\operatorname{Forb}(\Gamma, \mathcal{F})| & \leq \log _{2}\left(n^{n}\right)+\log _{2}\left(n^{n}\right)+\left(\max _{R \in \Gamma / \wp_{K}^{\leq}} \operatorname{ex}^{*}(R, \mathcal{F})+\frac{\varepsilon}{3}\right) n^{2} \\
& \leq\left(\max _{R \in \Gamma / \wp_{K}^{\leq}} \operatorname{ex}^{*}(R, \mathcal{F})+\varepsilon\right) n^{2} \quad\left(\operatorname{as~} \log _{2} n / n \leq \varepsilon / 3\right) .
\end{aligned}
$$

It remains to prove (8.6). To this end, fix $(\mathcal{V}, T)$ in the image of $\eta$ and let $R=\Gamma / \mathcal{V}$. Choose $S^{\prime} \in$ Forb $^{*}(R, \mathcal{F})$ such that $\mathbf{d}_{1}\left(T, S^{\prime}\right) \leq \varepsilon^{\prime 2}$. This is possible because $\mathcal{V}$ is an $\left(\varepsilon^{\prime 2}\right)$-recovering partition. Set $E_{1}=E_{1}\left(S^{\prime}\right)$ and partition $E_{0}\left(S^{\prime}\right)$ into $E_{0}^{+}:=\left\{(i, j) \in E_{0}\left(S^{\prime}\right): T(i, j)>\varepsilon^{\prime}\right\}$ and $E_{0}^{-}=$ $E_{0}\left(S^{\prime}\right) \backslash E_{0}^{+}$. Since there are $b(i, j):=\left(\begin{array}{l}\left|V_{i}\right|\left|V_{j}\right| R(i, j) \\ \left|V_{i}\right|\left|V_{j}\right| T(i, j)\end{array}\right)$ ways to choose $\left|V_{i}\right|\left|V_{j}\right| T(i, j)$ edges out of 
the $\left|V_{i}\right|\left|V_{j}\right| R(i, j)$ edges between $V_{i}$ and $V_{j}$ in $\Gamma$, we obtain

$$
\left|\eta^{-1}(\mathcal{V}, T)\right| \leq \prod_{1 \leq i<j \leq k} b(i, j) \leq \prod_{(i, j) \in E_{1}} \sqrt{b(i, j)} \prod_{(i, j) \in E_{0}^{+}} b(i, j) \prod_{(i, j) \in E_{0}^{-}} b(i, j) .
$$

Let us estimate the factors of (8.7):

We can bound each of the factors $b(i, j)$ of $E_{1}$ by $2^{R(i, j)\left|V_{i}\right|\left|V_{j}\right|}$. Since $\mathbf{d}_{1}\left(T, S^{\prime}\right) \leq \varepsilon^{\prime 2}$ we have $\left|E_{0}^{+}\right| \leq \varepsilon^{\prime} k^{2}$, as otherwise it would be the case that

$$
\mathbf{d}_{1}\left(T, S^{\prime}\right) \geq \sum_{(i, j) \in E_{0}^{+}}\left|T(i, j)-S^{\prime}(i, j)\right|>\left|E_{0}^{+}\right| \varepsilon^{\prime} \geq \varepsilon^{\prime 2} k^{2}
$$

which is a contradiction. Clearly, we have $\left|E_{0}^{-}\right| \leq k^{2}$. This allows us to upper bound each of the factors of $E_{0}^{+}$trivially by $2^{\left|V_{i} \| V_{j}\right|}$, and each of the factors of $E_{0}^{-}$by $2^{H\left(\varepsilon^{\prime}\right)\left|V_{i}\right|\left|V_{j}\right|}$ using (8.3).

Now let $S \in \operatorname{Forb}^{*}(R, \mathcal{F})$ be such that

$$
S(i, j)= \begin{cases}0 & \text { if }(i, j) \in E_{0}\left(S^{\prime}\right) \\ R(i, j) & \text { otherwise. }\end{cases}
$$

Taking the logarithm of (8.7) and using $\left|V_{i}\right|\left|V_{j}\right| \leq(n+k)^{2} / k^{2}$ we get

$$
\begin{aligned}
\log _{2}\left|\eta^{-1}(\mathcal{V}, T)\right| & \leq \sum_{(i, j) \in E_{1}} \frac{R(i, j)}{2}\left|V_{i}\right|\left|V_{j}\right|+\sum_{(i, j) \in E_{0}^{-}} H\left(\varepsilon^{\prime}\right)\left|V_{i}\right|\left|V_{j}\right|+\sum_{(i, j) \in E_{0}^{+}}\left|V_{i}\right|\left|V_{j}\right| \\
& \leq\left(\sum_{(i, j) \in E_{1}} \frac{R(i, j)}{2 k^{2}}+\sum_{(i, j) \in E_{0}^{-}} \frac{H\left(\varepsilon^{\prime}\right)}{k^{2}}+\sum_{(i, j) \in E_{0}^{+}} \frac{1}{k^{2}}\right)(n+k)^{2} \\
& \leq\left(\frac{1}{2 k^{2}} \sum_{(i, j) \in E_{1}} S(i, j)+H\left(\varepsilon^{\prime}\right)+\varepsilon^{\prime}\right)(n+k)^{2} .
\end{aligned}
$$

Now by using the fact that $S \in \operatorname{Forb}^{*}(R, \mathcal{F})$ and that $H\left(\varepsilon^{\prime}\right)+\varepsilon^{\prime} \leq \varepsilon / 6$ from equation (8.4), we infer

$$
\log _{2}\left|\eta^{-1}(\mathcal{V}, T)\right| \leq\left(\operatorname{ex}^{*}(R, \mathcal{F})+\frac{\varepsilon}{6}\right)(n+k)^{2} \leq\left(\operatorname{ex}^{*}(R, \mathcal{F})+\frac{\varepsilon}{3}\right) n^{2},
$$

which implies (8.6).

Proof of Theorem 8.2. Let $\mathcal{F}$ be a family of graphs such that $\operatorname{Forb}(\mathcal{F})$ is $f$-recoverable. Set $K=$ $f(\operatorname{poly}(\varepsilon))$ and $N=\operatorname{poly}(K, \varepsilon)$ given by Theorem 8.9 applied to $\varepsilon / 3$. Theorem 8.9 ensures that, whenever $\Gamma$ is a graph on $n \geq N$ vertices, we have

$$
\left|\frac{\log _{2}|\operatorname{Forb}(\Gamma, \mathcal{F})|}{n^{2}}-\max _{R \in \Gamma / \wp_{K}^{\leq}} \operatorname{ex}^{*}(R, \mathcal{F})\right| \leq \frac{\varepsilon}{3} .
$$

Let $\widehat{z}: \mathcal{G} \rightarrow \mathbb{R}$ be the graph parameter defined by $\widehat{z}(\Gamma)=\max _{R \in \Gamma / \wp_{K}^{\leqslant}} z^{*}(R)$, where $z^{*}(R)=$ $\operatorname{ex}^{*}(R, \mathcal{F})$. We claim that, given $R$ and $R^{\prime}$ in $\mathcal{G}^{*}(V)$, we have $\left|z^{*}(R)-z^{*}\left(R^{\prime}\right)\right| \leq \mathbf{d}_{1}\left(R, R^{\prime}\right)$. Indeed, assume without loss of generality that $z^{*}(R) \geq z^{*}\left(R^{\prime}\right)$ and fix a subgraph $S \leq R$ such that $S \in$ $\operatorname{Forb}_{>}(R, \mathcal{F})$ and $z^{*}(R)=\mathrm{e}(S) /|V|^{2}$. If $S \in \operatorname{Forb}_{\rtimes}\left(R^{\prime}, \mathcal{F}\right)$, we are done, so assume that this is not the case. Let $S^{\prime}$ be a subgraph of $S$ and $R^{\prime}$ maximizing e $\left(S^{\prime}\right)$, that is, $S^{\prime}(i, j)=\min \left\{S(i, j), R^{\prime}(i, j)\right\}$. 
Clearly,

$$
\mathrm{e}\left(S^{\prime}\right) \geq \mathrm{e}(S)-\frac{1}{2} \sum_{(i, j) \in V \times V}\left|R(i, j)-R^{\prime}(i, j)\right|=\mathrm{e}(S)-\frac{|V|^{2}}{2} \mathbf{d}_{1}\left(R, R^{\prime}\right),
$$

so that $0 \leq z^{*}(R)-z^{*}\left(R^{\prime}\right) \leq\left(\mathrm{e}(S)-\mathrm{e}\left(S^{\prime}\right)\right) /|V|^{2} \leq \frac{1}{2} \mathbf{d}_{1}\left(R, R^{\prime}\right)$.

This allows us to apply Theorem 7.1 to conclude that $\widehat{z}$ is estimable with sample complexity $q(\varepsilon)=$ $\operatorname{poly}(K, 1 / \varepsilon)$. Let $Q$ be chosen uniformly from all subsets of $V$ of size $q^{\prime}=\max \{q(\varepsilon / 3), N\}$ and set $\bar{\Gamma}=\Gamma[Q]$. It follows that, with probability at least $2 / 3$, we have $|\widehat{z}(\bar{\Gamma})-\widehat{z}(\Gamma)| \leq \varepsilon / 3$. By (8.8) we have $\left|n^{-2} \log _{2}\right| \operatorname{Forb}(\Gamma, \mathcal{F})|-\widehat{z}(\Gamma)| \leq \varepsilon / 3$. On the other hand, we can also apply (8.8) to $\bar{\Gamma}$ to obtain $\left|\widehat{z}(\bar{\Gamma})-q^{\prime-2} \log _{2}\right| \operatorname{Forb}(\bar{\Gamma}, \mathcal{F})|| \leq \varepsilon / 3$. By adding the last three inequalities, we get that

$$
\left|\frac{1}{n^{2}} \log _{2}\right| \operatorname{Forb}(\Gamma, \mathcal{F})\left|-\frac{1}{{q^{\prime}}^{2}} \log _{2}\right| \operatorname{Forb}(\bar{\Gamma}, \mathcal{F})|| \leq \varepsilon
$$

as required.

\subsection{Entropy and distance}

Given a monotone property $\operatorname{Forb}(\mathcal{F})$, the entropy $h_{\mathcal{F}}$ is actually closely related to the parameter $d_{\mathcal{F}}: \Gamma \mapsto \mathbf{d}_{1}(\Gamma, \operatorname{Forb}(\mathcal{F}))$. In fact, in this section we shall prove the following simple consequence of Theorem 8.2.

Corollary 8.10. Let $\operatorname{Forb}(\mathcal{F})$ be an $f$-recoverable graph property, for some function $f:(0,1] \rightarrow \mathbb{R}$. Then, for every $\varepsilon>0$ there is $n_{0}$ such that, for any graph $\Gamma$ with $|V(\Gamma)| \geq n_{0}$, the graph parameter $d_{\mathcal{F}}: \Gamma \mapsto \mathbf{d}_{1}(\Gamma, \operatorname{Forb}(\mathcal{F}))$ can be estimated within an additive error of $\varepsilon$ with sample complexity $\operatorname{poly}(f(\varepsilon / 6) / \varepsilon)$.

For a graph $\Gamma$ and a family of graphs $\mathcal{F}$, let

$\operatorname{ex}(\Gamma, \mathcal{F})$

$$
\operatorname{ex}(\Gamma, \mathcal{F})=\max \{\mathrm{e}(G): G \in \operatorname{Forb}(\Gamma, \mathcal{F})\}
$$

Moreover, let

$\overline{\operatorname{ex}}(\Gamma, \mathcal{F})$

$$
\overline{\operatorname{ex}}(\Gamma, \mathcal{F})=\frac{2}{n^{2}} \operatorname{ex}(\Gamma, \mathcal{F})
$$

be a normalized version of $\operatorname{ex}(\Gamma, \mathcal{F})$. Since $\operatorname{Forb}(\Gamma, \mathcal{F})$ is a monotone property, it is easy to see that the graph $G$ attaining the maximum in (8.9) must also minimize the distance $\mathbf{d}_{1}(\Gamma, G)$ over every $G \in \operatorname{Forb}(\Gamma, \mathcal{F})$. In other words, we must have

$$
\mathbf{d}_{1}(\Gamma, \mathcal{F})=\frac{2}{n^{2}} \mathrm{e}(\Gamma)-\overline{\operatorname{ex}}(\Gamma, \mathcal{F})
$$

Moreover, if $G$ is a graph attending the maximum of (8.10), then every subgraph of $G$ is also in $\operatorname{Forb}(\Gamma, \mathcal{F})$. Hence, it is clear that

$$
\frac{2}{n^{2}} \log _{2}|\operatorname{Forb}(\Gamma, \mathcal{F})|=: h_{\mathcal{F}}(\Gamma) \geq \overline{\operatorname{ex}}(\Gamma, \mathcal{F})
$$

By using the regularity method, Erdös, Frankl and Rödl [EFR86] showed that, for $\Gamma=K_{n}$ and $\mathcal{F}=\{F\}$, the trivial lower bound given in (8.12) is actually asymptotically tight, i.e., that 
$h_{\mathcal{F}}\left(K_{n}\right) \leq \overline{\mathrm{ex}}\left(K_{n}, \mathcal{F}\right)+o(1)$. One may extend these results to arbitrary graphs $\Gamma$ and arbitrary families $\mathcal{F}$, by using a stronger version of the regularity lemma, given in [AFKS00].

For completeness and to get a bound for $n$ that does not depend upon the regularity lemma, we show the following result, which is a consequence of Theorem 8.9.

Corollary 8.11. Let $\mathcal{F}$ be a family of graphs such that $\operatorname{Forb}(\mathcal{F})$ is $f$-recoverable for some function $f:(0,1] \rightarrow \mathbb{R}$. Then, for any $\varepsilon>0$, there exists $n_{0}=\operatorname{poly}(f(\operatorname{poly}(\varepsilon)))$ such that any graph $\Gamma$ of order $n \geq n_{0}$ satisfies

$$
\overline{\mathrm{ex}}(\Gamma, \mathcal{F}) \leq h_{\mathcal{F}}(\Gamma) \leq \overline{\operatorname{ex}}(\Gamma, \mathcal{F})+\varepsilon .
$$

Proof. By the above discussion, it suffices to prove the upper bound $h_{\mathcal{F}}(\Gamma) \leq \overline{\operatorname{ex}}(\Gamma, \mathcal{F})+\varepsilon$. Let $K$ be as in Theorem 8.9. Let $\mathcal{V}=\left\{V_{i}\right\}_{i=1}^{k} \in \wp_{K}^{\leqslant}$be an equipartition that maximizes $\operatorname{ex}^{*}(\Gamma / \mathcal{V}, \mathcal{F})$ over all equipartitions of $\Gamma$ of size at most $K$. Let $S \in \operatorname{Forb}_{\rightarrow}(G / \mathcal{V}, \mathcal{F})$ be such that e $(S)=$ $\left(k^{2} / 2\right) \mathrm{ex}^{*}(\Gamma / \mathcal{V}, \mathcal{F})$. By the maximality of $S$, we can assume without loss of generality that, for every $1 \leq i \leq j \leq k$, either $S(i, j)=G / \mathcal{V}(i, j)$ or $S(i, j)=0$. Furthermore, $S(i, i)=0$ for all $1 \leq i \leq k$. Consider the subgraph $G$ of $\Gamma$ that we get after removing every edge between $V_{i}$ and $V_{j}$, for each $1 \leq i \leq j \leq k$ satisfying $S(i, j)=0$. By construction we have $G / \mathcal{V}=S$, which implies that $G \in \operatorname{Forb}(\Gamma, \mathcal{F})$ by Proposition 8.4. Thus,

$$
\begin{array}{rl}
\overline{\operatorname{ex}}(\Gamma, \mathcal{F}) \geq \frac{2}{n^{2}} \mathrm{e}(G)=\frac{2}{n^{2}} \sum_{1 \leq i<j \leq k} & S(i, j) \frac{n^{2}}{k^{2}}=\frac{2}{k^{2}} \mathrm{e}(S) \\
& =\operatorname{ex}^{*}(\Gamma / \mathcal{V}, \mathcal{F})=\max _{R \in \Gamma / \wp_{K}^{\leq}} \operatorname{ex}^{*}(R, \mathcal{F}) \geq h_{\mathcal{F}}(\Gamma)-\varepsilon
\end{array}
$$

where the last inequality follows from Theorem 8.9.

Proof of Corollary 8.10. From Theorem 8.2 and Corollary 8.11, it follows that one can estimate $\overline{\operatorname{ex}}(\Gamma, \mathcal{F})$ with an additive error of $\frac{1}{2} \varepsilon$ and with sample complexity poly $(f(\operatorname{poly}(\varepsilon)))$ by simply estimating $h_{\mathcal{F}}(\Gamma)$ with an additive factor of $\varepsilon / 4$. Furthermore, $\Gamma \mapsto\left(2 / n^{2}\right) \mathrm{e}(\Gamma)$ can be estimated with an additive error of $\varepsilon / 2$ with sample complexity polynomial in $1 / \varepsilon$. Hence, it follows from (8.11) that $\mathbf{d}_{1}(\Gamma, \operatorname{Forb}(\mathcal{F}))$ is estimable with an additive error of $\varepsilon$ with sample complexity $\operatorname{poly}(f(\operatorname{poly}(\varepsilon)))$.

\subsection{Discussion}

In this chapter, we introduced the concept of $f$-recoverability of a graph property $\mathcal{P}$. Using this concept, and the fact that any monotone property $\mathcal{P}=\operatorname{Forb}(\mathcal{F})$ is recoverable for a function $f$ whose size is given by the Graph Removal Lemma, we found a probabilistic algorithm to estimate the number of $\mathcal{F}$-free subgraphs of a large graph $G$ whose sample complexity does not depend on regularity.

Being a new concept, little is known about $f$-recoverability itself, and we believe that it would be interesting to investigate this notion in more detail. For instance, in our proof that any monotone property $\operatorname{Forb}(\mathcal{F})$ is $f$-recoverable, we found $\varepsilon$-recovering partitions $\mathcal{V}$ that were $\gamma$-FK-regular (in fact, we showed that any such partition is $\varepsilon$-recovering), where $\gamma(\varepsilon)$ is chosen in such a way that the Removal Lemma applies. On the other hand, our discussion after Definition 8.6 implies that the property of being $r$-colorable is $\varepsilon$-recoverable with sample complexity $r / \varepsilon$, and thus we may 
find an $\varepsilon$-recovering partition whose size is less than the size required to ensure the existence of an FK-regular partition. It is natural to ask for properties that can be recovered by small partitions; more precisely, one could ask for a characterization of properties that are $f(\varepsilon)$-recoverable for $f(\varepsilon)$ polynomial in $1 / \varepsilon$.

Here, we restricted ourselves to monotone graph properties. We should mention that the parameter

$$
\widehat{h_{\mathcal{P}}}(\Gamma)=\frac{1}{|V(\Gamma)|^{2}} \log _{2}|\{G \leq \Gamma: G \in \mathcal{P}\}|
$$

might not even be estimable for arbitrary (non-monotone) properties $\mathcal{P}$. For instance, if $\mathcal{P}$ is the hereditary property of graphs having no independent sets of size three, then the complete graph $K_{n}$ and the graph $K_{n}-E\left(K_{3}\right)$, which is obtained from $K_{n}$ by removing the edges of a triangle, have quite a different number of spanning subgraphs satisfying $\mathcal{P}$, namely $2^{n^{2} / 4}$ and 0 , respectively, although their edit distance is negligible. It follows from $\left[\mathrm{BCL}^{+} 08\right.$, Theorem 6.1$]$ that $\widehat{h_{\mathcal{P}}}$ is not estimable.

Nevertheless, the definition of $f$-recoverability can be extended to cope with general hereditary properties, which, along with Theorem 7.1, provides a way of estimating other interesting hereditary parameters. In particular, this framework is used in Chapter 9 to estimate the distance to a given hereditary property by using a more direct route, instead of the indirect route given in Section 8.3, which is based on entropy. 


\section{Chapter 9}

\section{Estimation of distance to hereditary graph properties}

In this chapter we focus on hereditary graph properties. We recall that a graph property $\mathcal{P}$ is hereditary if it can be written as $\operatorname{Forb}^{\ominus}(\mathcal{F})$ for some (possibly infinite) family $\mathcal{F}$ of graphs - i.e., the property of graphs $G$ for which $\mathrm{p}^{\bullet}(F ; G)=0$ for every member $F \in \mathcal{F}$.

We recall that a hereditary property $\operatorname{Forb}^{\bullet}(\mathcal{F})$ being testable is equivalent to the existence of an Induced Removal Lemma for $\mathcal{F}$ (see Section 3.6.3). The results in this chapter rely on Lemma 3.28.

The goal of this chapter is to relate hereditary parameter estimation directly to the bounds of Induced Removal Lemmas by avoiding Szemerédi's Regularity Lemma, matching the spirit of Chapter 8 .

For every graph property $\mathcal{P}$, recall that the parameter

$$
\begin{aligned}
\mathbf{d}_{\mathcal{P}}: \mathcal{G} & \rightarrow[0,1] \\
\Gamma & \mapsto \mathbf{d}_{1}(\Gamma, \mathcal{P})
\end{aligned}
$$

that computes the distance from a graph property $\mathcal{P}$, i.e., the minimum of $\mathbf{d}_{1}(\Gamma, G)$ over all graphs $G \in \mathcal{P}$.

Remark 9.1 (notation). This chapter often adopts the convention of using the letter $\Gamma$ for graphs not necessarily in $\mathcal{P}$ and the letter $G$ for graphs in $\mathcal{P}$.

Theorem 9.2. Let $\mathcal{P}=\operatorname{Forb}(\mathcal{F})$ be any hereditary property. Then the graph parameter $\mathbf{d}_{\mathcal{P}}$ is estimable with sample complexity $s_{9.2}(\varepsilon)=\operatorname{EXP}\left\{\operatorname{poly}\left(\delta^{-M^{2}}, M, \log n_{0}\right)\right\}$, where $M, \delta$ and $n_{0}$ are as in Lemma 3.28 with input $\varepsilon / 3$ and $\mathcal{P}$.

Theorem 9.2 provides an upper bound on the sample complexity of estimating the distance to a hereditary property $\operatorname{Forb}(\mathcal{F})$, which solely depends on the upper bounds for the associated Removal Lemma. In particular, for families $\mathcal{F}$ that admit a Removal Lemma with sample complexity polynomial in $1 / \varepsilon$, our result states that the distance to $\operatorname{Forb}(\mathcal{F})$ can be estimated with a sample complexity that is exponential in a polynomial in $1 / \varepsilon$. Such families are currently actively sought after. Recent findings include the family consisting of a path on three edges [AF15], finite families containing a bipartite, a co-bipartite and a split graph [GS16] and the family of induced cycles of length at least four [dJdV19] (for $\mathcal{F}$ as in the last example, $\operatorname{Forb}(\mathcal{F})$ is the set of chordal graphs). 
This is a substantial improvement over previous approaches, like [FN07] and [ASS09], which rely on Szemerédi's Regularity Lemma and therefore provide bounds which are at least a tower of height polynomial in $1 / \varepsilon$.

The approach in Chapter 8 is based on a removal lemma for weighted graphs. Since we wished to arrive at a result involving classical (unweighted) removal lemmas, it was necessary to relate our weighted removal lemma with the classical removal lemma. In this chapter, so that we can use the classical (induced) removal lemma and its bounds directly, we take an alternative approach: instead of recoverable properties, we consider the notion of 'attestable' properties. Roughly speaking, a graph property $\mathcal{P}$ is $f$-attestable if every large graph $G \in \mathcal{P}$ is $\varepsilon$-close to admitting a partition $\mathcal{V}$ of its vertex set into at most $f(\varepsilon)$ classes that witnesses closeness to $\mathcal{P}$ (i.e., such that any graph that can be partitioned in the same way must be close to $\mathcal{P}$ ). Recall that, in contrast, recoverable refers to membership in $\mathcal{P}$. The proof of Theorem 9.2 consists of two steps. First we prove that $f$-attestable properties are estimable with sample complexity polynomial in $f$ (see Theorem 9.7). Then we show that hereditary properties are $f$-attestable, where $f$ is exponential in the bound given by Lemma 3.28 (see Theorem 9.9).

\subsection{Attestable properties}

As we will see, the reduced graph $\Gamma / \mathcal{V}$ can provide some information about $\Gamma$ if the number of classes of $\mathcal{V}$ is large enough (but still small with respect to the order of $\Gamma$ ), especially if $\mathcal{V}$ is a regular partition (in the sense of Frieze-Kannan).

Let $\mathcal{P}$ be a hereditary graph property and suppose that we want to estimate the parameter $\mathbf{d}_{\mathcal{P}}(\Gamma)$ for a graph $\Gamma$. Our aim is to show that $\mathbf{d}_{\mathcal{P}}(\Gamma)$ can be approximated by an optimization parameter over the set $\Gamma / \wp_{K}^{s}$, for some positive integer $K$. This will allow us to apply Corollary 7.2 to get sample complexity bounds for estimating $\mathbf{d}_{\mathcal{P}}$.

Next, for every weighted graph $R \in \mathcal{G}^{*}$, we will define the graph property $\Pi_{R}$ of being " $R$ reducible" by "essentially" putting $\Gamma \in \Pi_{R}$ if and only if $R \in \Gamma / \wp$.

Definition 9.3. For every $R \in \mathcal{G}^{*}([k])$ we define the property of $R$-reducible graph properties as

$\Pi_{R}$

$\Pi_{R}=\left\{\Gamma \in \mathcal{G}:\right.$ there is an equipartition $\mathcal{V}=\left\{V_{i}\right\}_{i=1}^{k}$ of $\Gamma$ for which $\left.\mathbf{d}_{1}(\Gamma / \mathcal{V}, R) \leq 2 / k\right\}$

Note that we actually allow graphs $\Gamma$ in $\Pi_{R}$ to have a partition $\mathcal{V}$ for which $\Gamma / \mathcal{V}$ is not exactly, but is very close to, $R$. This allows the definition to become more 'robust' - requiring a partition $\mathcal{V}$ for which $\Gamma / \mathcal{V}$ is exactly $R$ would make the property fail to contain graphs of arbitrarily large orders, because of divisibility issues. It is not hard to check that Definition 9.3 avoids such anomalies.

Proposition 9.4. For every $R \in \mathcal{G}^{*}([k])$, the property of being $R$-reducible contains graphs of every vertex size in $\left[k^{3 / 2}, \infty\right)$. In particular, the distance $\mathrm{d}\left(\Gamma, \Pi_{R}\right)$ is well-defined for every $\Gamma$ with at least $k^{3 / 2}$ vertices.

For a graph property $\mathcal{P}$ and for every $\varepsilon>0$, we define

$$
\mathcal{P}_{(\varepsilon)}^{*}=\left\{R \in \mathcal{G}^{*}: \mathbf{d}_{\mathcal{P}}(\Gamma) \leq \varepsilon \text { for all } \Gamma \in \Pi_{R}\right\}
$$

In other words, $\mathcal{P}_{(\varepsilon)}^{*}$ is the set of all reduced graphs $R$ that attest $\varepsilon$-closeness to $\mathcal{P}$, in the sense that 
if a graph $\Gamma$ admits a reduced graph close to $R$, then $\Gamma$ must be $\varepsilon$-close to $\mathcal{P}$. This motivates the following definition.

Definition 9.5 ( $f$-attestable). Given a function $f:(0,1] \rightarrow \mathbb{N}$, we say that a graph property $\mathcal{P}$ is $f$-attestable if, for any $\varepsilon>0$ and any graph $\Gamma \in \mathcal{P}$ with $|V(\Gamma)| \geq f(\varepsilon)^{3 / 2}$, there exists a partition $\mathcal{V}$ of $\Gamma$ into at most $f(\varepsilon)$ classes for which $G / \mathcal{V} \in \mathcal{P}_{(\varepsilon)}^{*}$.

For an integer $K>0$ and $\varepsilon>0$, we define the graph parameter $\mathbf{d}_{\mathcal{P}}^{(K, \varepsilon)}: \mathcal{G} \rightarrow[0,1]$ such that

$$
\mathbf{d}_{\mathcal{P}}^{(K, \varepsilon)}(\Gamma)=\min _{R \in \Gamma / \wp_{K}^{\leq}} \mathbf{d}_{1}\left(R, \mathcal{P}_{(\varepsilon)}^{*}\right)
$$

So if $\mathcal{P}$ is $f$-attestable, then by definition $\mathbf{d}_{\mathcal{P}}^{(K, \varepsilon)}(\Gamma)=0$ for $K=f(\varepsilon)$ and all graphs $\Gamma \in \mathcal{P}$ with $|V(\Gamma)| \geq K^{3 / 2}$. The next lemma shows that $\mathbf{d}_{\mathcal{P}}^{(K, \varepsilon)}$ is our desired optimization parameter.

Lemma 9.6. Let $\mathcal{P}$ be an $f$-attestable graph property for a function $f:(0,1] \rightarrow \mathbb{R}$. Fix $\varepsilon>0$ and let $K=f(\varepsilon)$. Then every graph $\Gamma \in \mathcal{G}(V)$ with $|V| \geq K^{3 / 2}$ satisfies $\left|\mathbf{d}_{\mathcal{P}}(\Gamma)-\mathbf{d}_{\mathcal{P}}^{(K, \varepsilon)}(\Gamma)\right| \leq \varepsilon$.

Before we prove Lemma 9.6, note that it asserts that $\mathbf{d}_{\mathcal{P}}$ satisfies the conditions of Corollary 7.2 (with $z^{*}=\mathbf{d}_{\mathcal{P}}^{(K, \varepsilon)}$ and $c=1$ ). As a consequence, we get the main result of this section.

Theorem 9.7. Let $\mathcal{P}$ be an $f$-attestable graph property, for a function $f:(0,1] \rightarrow \mathbb{R}$. Then, the graph parameter $\mathbf{d}_{\mathcal{P}}$ is estimable with sample complexity $s_{9.7}(\varepsilon)=\operatorname{poly}(f(\varepsilon / 3), 1 / \varepsilon)$.

Now we turn to the proof of Lemma 9.6.

Proof of Lemma 9.6. Fix $0<\varepsilon<1, K=f(\varepsilon)$. Let $V=[n]$ with $n \geq K^{3 / 2}$. We first show that $\mathbf{d}_{\mathcal{P}}^{(K, \varepsilon)}(\Gamma) \leq \mathbf{d}_{\mathcal{P}}(\Gamma)$. Let $G \in \mathcal{P}$ be a graph such that $\mathbf{d}_{1}(\Gamma, G)=\mathbf{d}_{\mathcal{P}}(\Gamma)$. Since $\mathcal{P}$ is $f$-attestable, we can fix an equipartition $\mathcal{V}=\left\{V_{i}\right\}_{i=1}^{k}$, with $k \leq K$, for which $G / \mathcal{V} \in \mathcal{P}_{(\varepsilon)}^{*}$. In particular, we have

$$
\begin{aligned}
\mathbf{d}_{\mathcal{P}}^{(K, \varepsilon)}(\Gamma) & \leq \mathbf{d}_{1}(\Gamma / \mathcal{V}, G / \mathcal{V})=\frac{1}{k^{2}} \sum_{(i, j) \in[k]^{2}} \frac{\left|\Gamma\left(V_{i}, V_{j}\right)-G\left(V_{i}, V_{j}\right)\right|}{\left|V_{i}\right|\left|V_{j}\right|} \\
& \leq \frac{1}{n^{2}} \sum_{(i, j) \in[k]^{2}} \sum_{\substack{u \in V_{i} \\
v \in V_{j}}}|\Gamma(u, v)-G(u, v)|=\mathbf{d}_{1}(\Gamma, G)=\mathbf{d}_{\mathcal{P}}(\Gamma) .
\end{aligned}
$$

Next, we proceed to show that $\mathbf{d}_{\mathcal{P}}(\Gamma) \leq \mathbf{d}_{\mathcal{P}}^{(K, \varepsilon)}(\Gamma)+\varepsilon$. Let $R \in \Gamma / \wp_{K}^{\leqslant}$and $S \in \mathcal{P}_{(\varepsilon)}^{*}$ be such that $\mathbf{d}_{1}(R, S)=\mathbf{d}_{\mathcal{P}}^{(K, \varepsilon)}(\Gamma)$. Let $k=|V(R)|$ and fix an equipartition $\mathcal{V}=\left\{V_{i}\right\}_{i=1}^{k}$ of $\Gamma$ such that $R=\Gamma / \mathcal{V}$. Let us construct a graph $G \in \Pi_{S}$ by modifying $\Gamma$ as follows. For each $1 \leq i<j \leq k$ such that $R(i, j)>S(i, j)$, we remove exactly $\left\lfloor(R(i, j)-S(i, j))\left|V_{i}\right|\left|V_{j}\right|\right\rfloor$ edges from $\Gamma$ between $V_{i}$ and $V_{j}$; if $S(i, j)>R(i, j)$, we add exactly $\left\lfloor(S(i, j)-R(i, j))\left|V_{i}\right|\left|V_{j}\right|\right\rfloor$ edges between $V_{i}$ and $V_{j}$ to $\Gamma$. Indeed we have $G \in \Pi_{S}$ as

$$
\begin{aligned}
\mathbf{d}_{1}(G / \mathcal{V}, S) & =\frac{1}{k^{2}} \sum_{(i, j) \in[k]^{2}}|G / \mathcal{V}(i, j)-S(i, j)| \\
& \leq \frac{1}{k^{2}}\left(k+\sum_{(i, j): i \neq j ; R(i, j)>S(i, j)}\left|\frac{\Gamma\left(V_{i}, V_{j}\right)}{\left|V_{i}\right|\left|V_{j}\right|}-\frac{\left\lfloor(R(i, j)-S(i, j))\left|V_{i}\right|\left|V_{j}\right|\right\rfloor}{\left|V_{i}\right|\left|V_{j}\right|}-S(i, j)\right|\right)
\end{aligned}
$$




$$
\begin{aligned}
& +\frac{1}{k^{2}}\left(k+\sum_{(i, j): i \neq j ; R(i, j)<S(i, j)}\left|\frac{\Gamma\left(V_{i}, V_{j}\right)}{\left|V_{i}\right|\left|V_{j}\right|}+\frac{\left\lfloor(S(i, j)-R(i, j))\left|V_{i}\right|\left|V_{j}\right|\right\rfloor}{\left|V_{i}\right|\left|V_{j}\right|}-S(i, j)\right|\right) \\
& \leq \frac{1}{k^{2}}\left(k+\sum_{(i, j) \in[k]^{2}} \frac{1}{\left|V_{i}\right|\left|V_{j}\right|}\right)=\frac{1}{k}+\frac{k^{2}}{n^{2}} \leq \frac{2}{k} .
\end{aligned}
$$

Moreover,

$$
\begin{aligned}
\mathbf{d}_{1}(\Gamma, G) & \leq \frac{1}{n^{2}} \sum_{(i, j) \in[k]^{2}}|R(i, j)-S(i, j)|\left|V_{i}\right|\left|V_{j}\right| \\
& =\frac{1}{k^{2}} \sum_{(i, j) \in[k]^{2}}|R(i, j)-S(i, j)| \\
& =\mathbf{d}_{1}(R, S) .
\end{aligned}
$$

Since $S \in \mathcal{P}_{(\varepsilon)}^{*}$, it follows that $\mathbf{d}_{1}(G, \mathcal{P}) \leq \varepsilon$. Hence, by the triangle inequality, $\mathbf{d}_{\mathcal{P}}(\Gamma) \leq \mathbf{d}_{1}(\Gamma, G)+\varepsilon \leq$ $\mathbf{d}_{\mathcal{P}}^{(K, \varepsilon)}(\Gamma)+\varepsilon$, as required.

Let $\mathcal{P}=\operatorname{Forb}(\mathcal{F})$ be a hereditary graph property. In the next section we show $\mathcal{P}$ is $f$-attestable with $f$ exponential on the parameters of the Induced Removal Lemma for $\mathcal{F}$.

For certain properties, however, one can get better bounds for $f$. A straightforward example would be, say, the property of graphs allowing a partition into $r$ sets (for $r$ fixed), that are either cliques of independent sets - such a property is poly $(1 / \varepsilon)$-attestable. Another simple example is Forb $^{\bullet}\left(P_{3}\right)$, which is equivalent to "being a union of cliques" — such a property is poly $(1 / \varepsilon)$-attestable.

It is also possible to show (see [FR20]) that $\operatorname{Forb}^{\bullet}\left(P_{4}\right)$ and chordality are poly $(1 / \varepsilon)$-attestable, and that $\operatorname{Forb}^{\boldsymbol{\Theta}}\left(C_{4}\right)$ is $K(\varepsilon)$-attestable, where $K(\varepsilon)=\exp (\exp (\operatorname{poly}(1 / \varepsilon)))$. In the latter case, one would get from Theorem 9.7 that $\mathbf{d}_{\mathrm{Forb}}{ }_{\left(C_{4}\right)}$ is estimable with sample complexity $\exp (\exp (\operatorname{poly}(1 / \varepsilon)))$. Recently, Fiat and Ron [FR20] were able to further improve the sample complexity required to estimate $\mathbf{d}_{\mathrm{Forb}} \boldsymbol{}_{\left(C_{4}\right)}$ to $\exp (\operatorname{poly}(1 / \varepsilon))$. In order to do so, they proved that the reduced graphs $R \in \mathcal{G}^{*}([K(\varepsilon)])$ that attest $\varepsilon$-closeness to $\operatorname{Forb}^{\boldsymbol{\Theta}}\left(C_{4}\right)$ can be taken each to be in the union of a small set of semi-homogeneous families of weighted graphs (see Section 5.1).

\subsection{Hereditary properties are attestable}

We recall that the homomorphism density $\mathrm{t}^{\bullet}(F ; R)$ of $F \in \mathcal{G}$ in $R \in \mathcal{G}^{*}$ is defined as the average homomorphism weight over all mappings $\varphi: V(F) \rightarrow V(R)$. Note that if $\Gamma$ is a graph, then $\mathrm{t}^{\bullet}(F ; \Gamma)$ is the probability that a random mapping $\varphi: V(F) \rightarrow V(\Gamma)$ is an induced homomorphism from $F$ to $\Gamma$. In particular, if $\Gamma \in \operatorname{Forb}(\{F\})$, then

$$
\mathrm{t}^{\bullet}(F ; \Gamma) \leq\left(\begin{array}{c}
|V(F)| \\
2
\end{array}\right) \cdot \frac{1}{|V(\Gamma)|},
$$

since a random mapping from $V(F)$ to $V(\Gamma)$ is not injective with probability at most $\left({ }^{|V(F)|}\right) /|V(\Gamma)|$. The next result shows that if $\mathrm{t}^{\bullet}(F ; \Gamma)$ is bounded away from zero, then so is $\mathrm{t}^{\bullet}(F ; \Gamma / \mathcal{V})$.

Lemma 9.8. Let $F$ and $\Gamma$ be graphs and $f=|V(F)|$. Then $\mathrm{t}^{\boldsymbol{\bullet}}(F ; \Gamma / \mathcal{V}) \geq \mathrm{t}^{\boldsymbol{\Theta}}(F ; \Gamma)^{f^{2}}$ holds for every equipartition $\mathcal{V}=\left\{V_{i}\right\}_{i=1}^{k}$ of $\Gamma$. 
Proof of Lemma 9.8. Suppose that $\mathcal{V}=\left\{V_{i}\right\}_{i=1}^{k}$ is an equipartition of $\Gamma$. Let $\Phi=V(\Gamma)^{V(F)}$ be the set of all functions from $V(F)$ to $V(\Gamma)$. For a mapping $\alpha: V(F) \rightarrow[k]$ we set

$$
\Phi_{\alpha}=\left\{\varphi \in \Phi: \varphi(u) \in V_{\alpha(u)} \text { for all } u \in V(F)\right\}
$$

Let $\varphi \in \Phi$ be chosen uniformly at random. For all mappings $\alpha: V(F) \rightarrow[k]$ and edges $u v \in E(F)$, we have

$$
\mathbb{P}\left(\operatorname{hw}_{\varphi}^{\bullet}(F ; \Gamma)=1 \mid \varphi \in \Phi_{\alpha}\right) \leq \mathbb{P}\left(\Gamma(\varphi(u), \varphi(v))=1 \mid \varphi \in \Phi_{\alpha}\right)=\Gamma / \mathcal{V}(\alpha(u), \alpha(v)),
$$

since $\Gamma / \mathcal{V}(\alpha(u), \alpha(v))$ is the probability that $\Gamma(x, y)=1$ when $x \in V_{\alpha(u)}$ and $y \in V_{\alpha(v)}$ are chosen uniformly (and independently) at random. Analogously, we also have

$$
\mathbb{P}\left(\operatorname{hw}_{\varphi}^{\mathbf{e}}(F ; \Gamma)=1 \mid \varphi \in \Phi_{\alpha}\right) \leq 1-\Gamma / \mathcal{V}(\alpha(u), \alpha(v))
$$

for all mappings $\alpha: V(F) \rightarrow[k]$ and all non-edges $u v \notin E(F)$. We can apply the last two inequalities to bound the homomorphism density $\mathrm{t}^{\bullet}(F ; \Gamma / \mathcal{V})$ from below as follows

$$
\begin{aligned}
& \mathrm{t}^{\bullet}(F ; \Gamma / \mathcal{V})=\sum_{\alpha: V(F) \rightarrow[k]} \frac{1}{k^{f}} \cdot\left(\prod_{u v \in E(F)} \Gamma / \mathcal{V}(\alpha(u), \alpha(v)) \prod_{u v \notin E(F)}(1-\Gamma / \mathcal{V}(\alpha(u), \alpha(v)))\right) \\
& =\sum_{\alpha: V(F) \rightarrow[k]} \mathbb{P}\left(\varphi \in \Phi_{\alpha}\right) \cdot\left(\prod_{u v \in E(F)} \Gamma / \mathcal{V}(\alpha(u), \alpha(v)) \prod_{u v \notin E(F)}(1-\Gamma / \mathcal{V}(\alpha(u), \alpha(v)))\right) \\
& \geq \sum_{\alpha: V(F) \rightarrow[k]} \mathbb{P}\left(\varphi \in \Phi_{\alpha}\right) \cdot \mathbb{P}\left(\operatorname{hw}_{\varphi}^{\Theta}(F ; \Gamma)=1 \mid \varphi \in \Phi_{\alpha}\right)^{\left(\begin{array}{l}
f \\
2
\end{array}\right) .}
\end{aligned}
$$

Since $x \mapsto x^{\left(\begin{array}{l}f \\ 2\end{array}\right)}$ is convex for every $x \geq 0$, we get (Lemma 2.1) that

$$
\mathrm{t}^{\bullet}(F ; \Gamma / \mathcal{V}) \geq\left(\sum_{\alpha: V(F) \rightarrow[k]} \mathbb{P}\left(\varphi \in \Phi_{\alpha}\right) \cdot \mathbb{P}\left(\operatorname{hw}_{\varphi}^{\bullet}(F ; \Gamma)=1 \mid \varphi \in \Phi_{\alpha}\right)\right)^{\left(\begin{array}{l}
f \\
2
\end{array}\right)} \geq \mathrm{t}^{\bullet}(F ; \Gamma)^{f^{2}}
$$

as desired.

Note that the converse of Lemma 9.8 does not hold in general. For instance, the complete bipartite graph $\Gamma=K_{n, n}$ satisfies $\mathrm{t}^{\bullet}\left(K_{3} ; K_{n, n}\right)=0$, but $\mathrm{t}^{\boldsymbol{\bullet}}\left(K_{3} ; K_{n, n} / \mathcal{V}\right)$ is close to $1 / 8$ if $\mathcal{V}$ is a random equipartition of large size. However, $\mathrm{t}^{\boldsymbol{}}(F ; \Gamma)$ and $\mathrm{t}^{\boldsymbol{}}(F ; \Gamma / \mathcal{V})$ are known to be close, provided $\mathcal{V}$ is a Frieze-Kannan-regular partition (see Lemma 3.22).

Now we are ready to show that hereditary graph properties are attestable.

Theorem 9.9. For every family $\mathcal{F}$ of graphs, the property $\operatorname{Forb}(\mathcal{F})$ is $f$-attestable for $f(\varepsilon)=$ $2^{\text {poly }\left(\delta^{-M^{2}}, M, \log n_{0}\right)}$, where $\delta, M$ and $n_{0}$ are as in Lemma 3.28 with input $\mathcal{F}$ and $\varepsilon$.

Proof. Let $\delta, M$ and $n_{0}$ be as in Lemma 3.28 with inputs $\mathcal{F}$ and $\varepsilon$. Let $K$ be as in Lemma 3.15 with input

$$
k_{0}=\max \left\{n_{0}, \frac{2}{\delta}, \frac{16 M^{2}}{\delta^{M^{2}}}\right\} \quad \text { and } \quad \gamma=\frac{\delta^{M^{2}}}{16 M^{2}} .
$$

Note that $K=2^{\operatorname{poly}\left(\delta^{-M^{2}}, M, \log n_{0}\right)}$. Let $G \in \operatorname{Forb}(\mathcal{F})$ be a graph with $n \geq K$ vertices. We claim 
that if $\mathcal{V}$ is a $\gamma$-FK-regular equipartition of $G$ into $k_{0} \leq k \leq K$ classes, then $R:=G / \mathcal{V} \in \mathcal{P}_{(\varepsilon)}^{*}$. This will prove the theorem for $f(\varepsilon)=K$.

Suppose by contradiction that there is a graph $H \in \Pi_{R}$ such that $\mathbf{d}_{1}(H, \operatorname{Forb}(\mathcal{F}))>\varepsilon$. Since $|V(H)| \geq k_{0} \geq n_{0}$, Lemma 3.28 asserts there must be a graph $F \in \mathcal{F}$, with $|V(F)| \leq M$, for which $\mathrm{t}^{\bullet}(F ; H) \geq \delta$.

As $H \in \Pi_{R}$, there is a partition $\mathcal{V}^{\prime}$ of $H$ into $k$ classes for which $\mathbf{d}_{1}\left(H / \mathcal{V}^{\prime}, R\right) \leq 2 / k$. In particular, we must also have $\left\|H / \mathcal{V}^{\prime}-R\right\|_{\square} \leq \mathbf{d}_{1}\left(H / \mathcal{V}^{\prime}, R\right) \leq 2 / k$. Therefore, Lemma 3.20 implies

$$
\mathrm{t}^{\boldsymbol{\Theta}}(F ; R) \geq \mathrm{t}^{\boldsymbol{\Theta}}\left(F ; H / \mathcal{V}^{\prime}\right)-4 M^{2}\left\|H / \mathcal{V}^{\prime}-R\right\|_{\square} \geq \mathrm{t}^{\boldsymbol{\Theta}}\left(F ; H / \mathcal{V}^{\prime}\right)-\frac{8 M^{2}}{k} \geq \mathrm{t}^{\boldsymbol{\bullet}}\left(F ; H / \mathcal{V}^{\prime}\right)-\frac{\delta^{M^{2}}}{2}
$$

Moreover, we must have $\mathrm{t}^{\bullet}\left(F ; H / \mathcal{V}^{\prime}\right) \geq \delta^{M^{2}}$ by Lemma 9.8 . We conclude that

$$
\mathrm{t}^{\bullet}(F ; R) \geq \frac{1}{2} \delta^{M^{2}}
$$

On the other hand, $R$ is the reduced graph of $G$ with respect to a $\gamma$-FK-regular partition. Therefore, by Lemma 3.22, we have

$$
\mathrm{t}^{\bullet}(F ; G) \geq \mathrm{t}^{\boldsymbol{\Theta}}(F ; R)-4 M^{2} \gamma \geq \frac{\delta^{M^{2}}}{2}-4 M^{2} \gamma \geq \frac{\delta^{M^{2}}}{4} .
$$

But this contradicts $(9.1)$, which asserts that $\mathrm{t}^{\bullet}(F ; G)$ is at most $\left(\begin{array}{c}M \\ 2\end{array}\right) \frac{1}{n} \leq \frac{M^{2}}{k_{0}} \leq \frac{\delta^{M^{2}}}{16}$.

Note that Theorem 9.2 follows from Theorems 9.7 and 9.9 .

\subsection{Shedding some light on shadows}

Recall that, given a graph $G$ and an equipartition $\mathcal{V}$ of $G$, the quotient graph $G / \mathcal{V}$ is just a more concise representation of $G_{\mathcal{V}}$ - the orthogonal projection ("shadow") of $G$ onto the lower dimensional space of $\mathcal{V}$-constant weighted graphs. If one can approximate the value of a graph parameter $f(G)$ by just inspecting the shadows of $G$ on the bounded dimension spaces $\wp_{K}^{\leqslant}$, for some integer $K$, then $f$ is estimable (because those shadows of $G$ are essentially the same as the shadows of a sample $G[Q]$; see Theorem 5.1).

The strategy we used in Chapters 8 and 9 to obtain better bounds for estimating $\mathbf{d}_{\mathcal{P}}$ relied on the fact that, for every $G \in \mathcal{P}$, there is a single partition $\mathcal{V}$ for which the shadow of $G$ witnesses proximity of $G$ to $\mathcal{P}$ (either by the concept of being $f$-recoverable or $f$-attestable).

Although this strategy works for monotone and hereditary properties, it cannot work in general. Consider the following graph property:

$$
\mathcal{P}=\left\{G: \mathrm{p}\left(K_{3} ; G\right) \geq \frac{1}{9}\right\} .
$$

The property $\mathcal{P}$ above is known to be easily-testable (see [GS16]) — properties of this kind (given by linear constraints of graph densities) will be considered in Chapter 10. On the other hand, we have the following fact.

Fact 9.10. $\mathcal{P}$ is not $f$-attestable for any function $f$. 
Proof. Indeed, let $G=G_{n, \frac{1}{2}}$ be a typical random graph. Then, $\mathrm{p}\left(K_{3} ; G\right)=\frac{1}{8}+O(1 / n) \geq \frac{1}{9}$ for every large enough $n$. In particular, we have $G \in \mathcal{P}$. But for any given $K$, every reduced graph $R \in G / \wp_{K}^{\leqslant}$has $R(i, j)=\frac{1}{2} \pm o(1)$ for every $i j$. Therefore, no single $R \in G / \wp_{K}^{\leqslant}$can attest proximity to $\mathcal{P}$. Indeed, note that the complete bipartite graph $H=K_{\frac{n}{2}}, \frac{n}{2}$ is far from satisfying $\mathcal{P}$, but it has a partition $\mathcal{W}$ satisfying $H / \mathcal{W}(i, j)=\frac{1}{2} \pm o(1)$ : it suffices to consider a typical random partition $\mathcal{W}$.

The fact above show us that considering a single $R \in G / \wp_{K}^{\leq}$of a graph $G \in \mathcal{P}$ is not powerful enough to approximate $\mathbf{d}_{\mathcal{P}}(G)$. However, it might still be the case that the whole set $G / \wp_{K}^{\leqslant}$carries enough information about $\mathbf{d}_{\mathcal{P}}(G)$.

For example, note that knowing that every partition of a graph $G$ into at most $K$ classes satisfies $G / \mathcal{W}(i, j)=\frac{1}{2} \pm o(1)$ tell us a lot about $G$. In fact, for such a graph $G$ any partition $\mathcal{V}=\left\{V_{i}\right\}_{i=1}^{k}$ of size $k \ll K$ must be very regular - since there is no refinement of $\mathcal{V}$ that increases $\left\|G_{\mathcal{V}}\right\|_{2}^{2}$ substantially. In particular, that implies that $G \in \mathcal{P}$.

Summing up, approximating by quotient graphs might still be a good way of estimating parameters, but it might be the case that one has to consider the whole set of quotients, instead of just one. In that sense, it might be interesting to investigate algorithms which estimate graph parameters by only having quot-access (see Definition 7.3) to the input graph. 


\section{Chapter 10}

\section{On graph properties admitting a proximity oblivious tester}

In this chapter we study a special kind of tester, called proximity oblivious tester, which is defined as follows.

Definition 10.1. A proximity oblivious tester $(P O T)$ for a graph property $\mathcal{P}$ is an randomized decision algorithm $\mathcal{T}_{\text {Por }}$ for which there are constants $q \in \mathbb{N}, c \in(0,1]$ and a function $f:(0,1] \rightarrow(0,1]$ satisfying the following for any $\varepsilon>0$. For any given input graph $G$, the tester $\mathcal{T}_{\text {рот }}$ makes $q$ queries to $G$ (note that $q$ is not a function $\varepsilon$ ) and

(i) if $G$ satisfies $\mathcal{P}$, then $\mathcal{T}_{\text {рот }}$ ACCEPTS $G$ with probability at least $c$.

(ii) if $G$ is $\varepsilon$-far from $\mathcal{P}$, then $\mathcal{T}_{\text {por }}$ ACCEPTS $G$ with probability at most $c-f(\varepsilon)$.

The following simple proposition shows that the definition of a POT is stronger than the definition of a usual tester (Definition 4.1).

Proposition 10.2. If a property $\mathcal{P}$ has a POT, then for every $\varepsilon>0$, there is an $\varepsilon$-tester for $\mathcal{P}$.

Proof. Let $\varepsilon>0$ be given and let $\mathcal{T}_{\text {por }}$ be a POT for $\mathcal{P}$. Consider an algorithm $\mathcal{T}$ that, given an input graph $G$, invokes $\mathcal{T}(G)$ successively $k=\left\lceil 4 / f(\varepsilon)^{-2}\right\rceil$ times, and then accepts $G$ if and only if $\mathcal{T}$ accepted $G$ in at least $\left(c-\frac{1}{2} f(\varepsilon)\right) k$ of those times. It is not difficult to see that $\mathcal{T}$ is an $\varepsilon$-tester for $\mathcal{P}$.

Indeed, for every $i \in[k]$, let $\left\{X_{i}\right\}_{i=1}^{k}$ be the random variable indicating if $G$ is accepted in the $i$-th run of $\mathcal{T}_{\text {рот }}$ and let $S_{k}=\sum_{i=1}^{k} X_{i}$. If $G \in \mathcal{P}$, then $\mathbb{E}\left(S_{k}\right)=\sum_{i=1}^{k} \mathbb{E}\left(X_{i}\right) \geq c k$. It follows that

$$
\mathbb{P}(\mathcal{T} \text { rejects } G)=\mathbb{P}\left(S_{k}<c k-\frac{1}{2} f(\varepsilon) k\right) \leq \mathbb{P}\left(S_{k} \leq \mathbb{E}\left(S_{k}\right)-\frac{1}{2} f(\varepsilon) k\right) .
$$

On the other hand, if $G$ is $\varepsilon$-far from $\mathcal{P}$, then $\mathbb{E}\left(S_{k}\right)=\sum_{i=1}^{k} \mathbb{E} X_{i} \leq c k-f(\varepsilon) k$. It follows that

$$
\mathbb{P}(\mathcal{T} \text { accepts } G)=\mathbb{P}\left(S_{k} \geq c k-\frac{1}{2} f(\varepsilon) k\right) \leq \mathbb{P}\left(S_{k} \geq \mathbb{E}\left(S_{k}\right)+\frac{1}{2} f(\varepsilon) k\right) .
$$


On both cases, Lemma 2.3 implies $\mathcal{T}$ makes the "wrong" decision with probability at most

$$
\exp \left\{-\frac{f(\varepsilon)^{2}}{2} \frac{1}{k}\right\} \leq e^{-2}<\frac{1}{3},
$$

i.e. $\mathcal{T}$ is indeed an $\varepsilon$-tester for $\mathcal{P}$.

POTs were introduced by Goldreich and Ron [GR11], who studied one-sided-error POTs, namely POTs that accept every input which satisfies the property with probability 1 (this corresponds to having $c=1$ in the Definition 10.1). Later, Goldreich and Shinkar [GS16] studied general (two-sided-error) POTs in several settings, including general boolean functions, dense graphs and bounded degree graphs. For the dense graph model, they designed a POT for the property of being $\alpha$-regular (for a given $\alpha \in(0,1)$ ), as well as related for properties. Moreover, they considered properties of the following form.

Definition 10.3. Given an integer $h \geq 2$, a rational number $b$ and a function $w: \mathcal{G}([h]) \rightarrow[0,1]$, from graphs on $h$ vertices (up to isomorphisms), the property $\mathcal{D}_{h, w, b}$ is defined as

$$
\mathcal{D}_{h, w, b}=\left\{G: \sum_{H} w(H) \cdot \mathrm{p}^{\bullet}(H ; G) \leq b\right\}
$$

Throughout this chapter, a tuple $(h, w, b)$ will always denote an integer $h \geq 2$, a function $w: \mathcal{G}([h]) \rightarrow \mathbb{Q}$ and $b \in \mathbb{Q}$. The value $w(H)$ will sometimes be denoted by $w_{H}$.

Definition 10.4. We say that a tuple $(h, w, b)$ has the removal property if there is a function $f:(0,1] \rightarrow(0,1]$ such that for every $\varepsilon \in(0,1)$ and for every graph $G$, if $G$ is $\varepsilon$-far from $\mathcal{D}_{h, w, b}$ then $\sum_{H} w(H) \cdot \mathrm{p}^{\bullet}(H ; G) \geq b+f(\varepsilon)$.

Goldreich and Shinkar [GS16] observed that if $(h, w, b)$ has the removal property then $\mathcal{D}_{h, w, b}$ admits an input-size oblivious POT. Indeed, given an input graph $G$, the POT works by sampling a random induced subgraph $G[S]$ of $G$ of order $h=|S|$, and then rejecting with probability $w_{G[S]}$. If $G$ satisfies $\mathcal{D}_{h, w, b}$, then by the definition of this property, $G$ is rejected with probability $\sum_{H} w(H) \cdot \mathrm{p}^{\bullet}(H ; G) \leq b$. On the other hand, if $G$ is $\varepsilon$-far from the property then by the removal property, $G$ is rejected with probability $\sum_{H} w(H) \cdot \mathrm{p}^{\mathbf{o}}(H ; G) \geq b+f(\varepsilon)$. Thus, Definition 10.1 is satisfied with $c=1-b$.

The first result of this chapter is a converse of the above statement, showing that if $\mathcal{D}_{h, w, b}$ has an input-size oblivious POT, then $(h, w, b)$ has the removal property.

Theorem 10.5. Let $(h, w, b)$ be a tuple. If $\mathcal{D}_{h, w, b}$ has an input-size oblivious POT then $(h, w, b)$ has the removal property.

As a corollary of the above theorem, we infer that if one "representation" of a property as $\mathcal{D}_{h, w, b}$ has the removal property, then all such representations have the removal property. This is stated in the following corollary.

Corollary 10.6. Let $(h, w, b)$ and $\left(h^{\prime}, w^{\prime}, b^{\prime}\right)$ be tuples such that $\mathcal{D}_{h, w, b}=\mathcal{D}_{h^{\prime}, w^{\prime}, b^{\prime}}$. Then $(h, w, b)$ has the removal property if and only if $\left(h^{\prime}, w^{\prime}, b^{\prime}\right)$ has the removal property.

Remark 10.7. Theorem 10.5 contradicts the statement of Proposition 3.14 in [GS16], which states that there is a tuple $(h, w, b)$ such that $\mathcal{D}_{h, w, b}$ has a POT but $(h, w, b)$ does not have the removal property. We believe that the proof of this proposition is wrong. This is argued in Section 10.3. 
The following conjecture was raised by Goldreich and Shinkar in [GS16].

Conjecture 10.8 ([GS16, Problem 3.11]). Every property $\mathcal{D}_{h, w, b}$ has a POT.

Our next theorem disproves the above conjecture by showing that there are properties $\mathcal{D}_{h, w, b}$ that are not testable at all (let alone testable using a POT).

Theorem 10.9. Let $K_{4}$ denote the complete graph on 4 vertices, $D_{4}$ the diamond graph (i.e. $K_{4}$ minus an edge), $P_{3}$ the graph on 4 vertices containing a path of length 2 and an isolated vertex, $C_{4}$ the 4-cycle, $P_{4}$ the path on 4 vertices and $K_{1,3}$ the star on 4 vertices (see below). Let $h=4$, $b=\frac{5}{16}$ and let $w_{H}$ be the following weight function assigning a non-negative weight to each (up to isomorphisms) graph $H$ on 4 vertices.

\begin{tabular}{|c|c|c|c|c|c|c|c|c|c|c|c|}
\hline$H:$ & $\mathbb{D}_{0}^{0}$ & $\begin{array}{ll}\circ & \circ \\
\circ & \circ \\
\end{array}$ & $\stackrel{0}{0}$ & $0 \%$ & $\begin{array}{ll}0 \\
0 & 0 \\
\end{array}$ & 20 & $\Sigma_{0}^{0}$ & i! & $\Omega_{0}^{\circ}$ & ${ }^{\circ}$ & $\stackrel{0}{\circ}$ \\
\hline$H:$ & $K_{4}$ & $\overline{K_{4}}$ & $D_{4}$ & $\overline{D_{4}}$ & $P_{3}$ & $\overline{P_{3}}$ & $C_{4}$ & $\overline{C_{4}}$ & $K_{1,3}$ & $\overline{K_{1,3}}$ & $P_{4}$ \\
\hline$w_{H}:$ & 1 & $\frac{1}{2}$ & $\frac{5}{12}$ & $\frac{5}{12}$ & $\frac{1}{6}$ & $\frac{1}{3}$ & $\frac{1}{2}$ & $\frac{1}{3}$ & $\overline{4}$ & $\frac{1}{4}$ & $\frac{1}{4}$ \\
\hline
\end{tabular}

Then, the property

$$
\mathcal{D}_{h, w, b}:=\left\{G: \sum_{H:|V(H)|=4} w_{H} \cdot \mathrm{p}^{\bullet}(H ; G) \leq \frac{5}{16}\right\}
$$

is not testable.

Given the above theorem it is natural to ask if every property $\mathcal{D}_{h, w, b}$ can at least be tested using $o\left(n^{2}\right)$ edge-queries. We leave this as an open problem.

Chapter overview: The rest of this chapter is organized as follows. In Section 10.1 we prove Theorem 10.5. The proof of Theorem 10.9 appears in Section 10.2. The main idea behind its proof is to show that there exists a property $\mathcal{D}_{h, w, b}$ (specifically, the one defined in Eq. (10.1)) which encodes the property of being a quasi-random graph. More precisely, we show that if a graph $G$ satisfies the property given by Eq. (10.1), then its edge density must be roughly $1 / 2$ and its $C_{4}$ density roughly $1 / 16$, which is known to imply that $G$ must be quasi-random (see [CGW89] and Lemma 3.8). It is then not hard to show that the property defined by (10.1) cannot be tested using a constant number of queries. Finally, in Section 10.3 we address the discrepancy between Theorem 10.5 and one of the results of [GS16].

\subsection{Proof of Theorem $\mathbf{1 0 . 5}$}

In this section we prove Theorem 10.5 and Corollary 10.6. We will need the following auxiliary lemma.

Lemma 10.10. Let $\Pi$ be a graph property, and suppose that $\Pi$ has a canonical size-oblivious $\varepsilon$ tester $\mathcal{T}$ with sample complexity $s=s(\varepsilon)$ (and success probability $\frac{2}{3}$ ). Then for every $n \geq s^{4}$ and for every n-vertex graph $G$ which is $\varepsilon$-far from $\Pi$, the following holds. For $U$ chosen uniformly at random from $\left(\begin{array}{c}V(G) \\ s^{4}\end{array}\right)$, we have $\mathbb{P}(G[U] \in \Pi)=e^{-\Omega(s)}$. 
Proof. Let $\mathcal{A}$ be the family of all $s$-vertex graphs $A$ such that $\mathcal{T}$ accepts if it sees a subgraph isomorphic to $A$. For a graph $G$, we say that a sequence of subsets $S_{1}, \ldots, S_{s} \in\left({ }^{V(G)}{ }_{s}\right)$ is $\operatorname{good}$ if $G\left[S_{i}\right] \in \mathcal{A}$ for at least half of the values of $1 \leq i \leq s$; otherwise $S_{1}, \ldots, S_{s}$ is bad. For a sequence of vertices $W=$ $\left(x_{1}, \ldots, x_{s^{2}}\right)$, we say that $W$ is good (bad) if $\left\{x_{1}, \ldots, x_{s}\right\},\left\{x_{s+1}, \ldots, x_{2 s}\right\}, \ldots,\left\{x_{s^{2}-s+1}, \ldots, x_{s^{2}}\right\}$ is good (respectively bad). For a random $S \in\left(\begin{array}{c}V(G) \\ s\end{array}\right)$, if $G \in \Pi$ then $\mathbb{P}(G[S] \in \mathcal{A}) \geq \frac{2}{3}$, and if $G$ is $\varepsilon$-far from $\Pi$ then $\mathbb{P}(G[S] \in \mathcal{A}) \leq \frac{1}{3}$. Using a standard Chernoff-type bound, one can show that the following holds for $S_{1}, \ldots, S_{s} \in\left(\begin{array}{c}V(G) \\ s\end{array}\right)$ chosen uniformly at random and independently.

(i) If $G \in \Pi$ then $S_{1}, \ldots, S_{s}$ is good with probability at least $1-e^{-C s}$.

(ii) If $G$ is $\varepsilon$-far from $\Pi$ then $S_{1}, \ldots, S_{s}$ is bad with probability at least $1-e^{-C s}$.

In both items above, $C$ is an absolute constant.

The probability that $S_{i} \cap S_{j} \neq \emptyset$ for some $1 \leq i<j \leq s$ is at most $\left(\begin{array}{c}s \\ 2\end{array}\right) \frac{s^{2}}{n}<\frac{1}{2}$, where we used the assumption $n \geq s^{4}$. So $\left|S_{1} \cup \cdots \cup S_{s}\right|=s^{2}$ with probability at least $\frac{1}{2}$. Conditioned on the event that $S_{1}, \ldots, S_{s}$ are pairwise disjoint, the set $S:=S_{1} \cup \cdots \cup S_{s}$ has the distribution of an element of $\left(\begin{array}{c}V(G) \\ s^{2}\end{array}\right)$ chosen uniformly at random. Thus, a random sequence of vertices $W=\left(x_{1}, \ldots, x_{s^{2}}\right)$ chosen without repetitions satisfies the following.

(i) If $G$ satisfies $\Pi$ then $W$ is good with probability at least $1-2 e^{-C s}$.

(ii) If $G$ is $\varepsilon$-far from $\Pi$ then $W$ is bad with probability at least $1-2 e^{-C s}$.

Now let $G$ be a graph on $n \geq s^{4}$ vertices which is $\varepsilon$-far from $\Pi$. Consider a random pair $(U, W)$, where $U$ is chosen uniformly at random from $\left(\begin{array}{c}V(G) \\ s^{4}\end{array}\right)$, and $W=\left(x_{1}, \ldots, x_{s^{2}}\right)$ is a sequence of vertices sampled randomly without repetition from $U$. Note that $W$ is distributed as a uniform sequence of vertices of $G$ sampled without repetition. Thus, $\mathbb{P}(W$ is good $) \leq 2 e^{-C s}$. On the other hand, if $G[U] \in \Pi$, then $\mathbb{P}(W$ is good $\mid U) \geq 1-2 e^{-C s}$. By combining these two facts, we see that

$$
\mathbb{P}(G[U] \in \Pi) \leq \frac{2 e^{-C s}}{1-2 e^{-C s}} \leq 4 e^{-C s} \leq e^{-\Omega(s)} .
$$

Proof of Theorem 10.5. As stated in Proposition 10.2, a POT for $\mathcal{D}_{h, w, b}$ can be used to obtain a standard tester for $\mathcal{D}_{h, w, b}$ by invoking the POT an appropriate number of times. Moreover, it is clear that if the POT is input-size oblivious, then so is the resulting tester. Next, we apply the transformation of Goldreich and Trevisan [GT03] to get a canonical tester $\mathcal{T}$ for $\mathcal{D}_{h, w, b}$. Since this transformation preserves the property of being input-size oblivious, $\mathcal{T}$ is input size oblivious, and hence satisfies the condition of Lemma 10.10.

Denote by $s=s(\varepsilon)$ the sample complexity of $\mathcal{T}$. We may and will assume that $s$ is large enough as a function of the parameters $h$ and $b$.

Let us denote $z(G)=\sum_{H} w_{H} \cdot \mathrm{p}^{\bullet}(H ; G)$. By multiplying the inequality $\sum_{H} w_{H} \cdot \mathrm{p}^{\bullet}(H ; G) \leq b$ by an appropriate integer, we can assume that $b$ is an integer and that $w_{H}$ is an integer for every $H$. Denote by $s=s(\varepsilon)$ the sample complexity of $\mathcal{T}$. We may and will assume that $s$ is large enough as a function of the parameters $h$ and $b$. By Lemma 10.10, a randomly chosen $U \in\left(\begin{array}{c}V(G) \\ s^{4}\end{array}\right)$ satisfies $G[U] \in \mathcal{D}_{h, w, b}$ with probability at most $e^{-\Omega(s)}$. Observe that if a $k$-vertex graph $K$ does not satisfy $\mathcal{D}_{h, w, b}$, then necessarily

$$
z(K)=\sum_{H} w_{H} \cdot \mathrm{p}^{\bullet}(H ; K) \geq b+\left(\begin{array}{l}
k \\
h
\end{array}\right)^{-1}>b+k^{-h},
$$


as $b$ and all weights $w_{H}$ are integers. Thus, if $G[U] \notin \mathcal{D}_{h, w, b}$ then

$$
z(G[U])>b+|U|^{-h}=b+s^{-4 h} .
$$

Observe that $z(G)$ is the average of $z(G[U])$ over all $U \in\left(\begin{array}{c}V(G) \\ s^{4}\end{array}\right)$. Thus, using the guarantees of Lemma 10.10, we obtain

$$
z(G) \geq\left(1-e^{-\Omega(s)}\right)\left(b+s^{-4 h}\right)>b+\frac{1}{2} s^{-4 h},
$$

where the last inequality holds provided that $s$ is large enough as a function of $h$ and $b$. So we may take the function $f$ in Definition 10.4 to be $f(\varepsilon)=\frac{1}{2} s(\varepsilon)^{-4 h}$. This completes the proof.

Proof of Corollary 10.6. We have established that $(h, w, b)$ satisfies the removal property if and only if $\mathcal{D}_{h, w, b}$ has an input-size oblivious POT. The "only if" part was explained in the introduction (see also [GS16]), and the "if" part is the statement of Theorem 10.5. It is now clear that the corollary holds.

\subsection{Proof of Theorem $\mathbf{1 0 . 9}$}

Let $\mathcal{D}_{h, w, b}$ be as in the statement Theorem 10.9. Denote

$$
z(G):=\sum_{H:|V(H)|=4} w_{H} \cdot \mathrm{p}^{\bullet}(H ; G)
$$

for every graph $G$ of order at least 4 . Under this notation, $\mathcal{D}_{h, w, b}=\{G: z(G) \leq b\}$. The following lemma gives a simpler description of $\mathcal{D}_{h, w, b}$.

Lemma 10.11. $\mathcal{D}_{h, w, b}=\{G: \varphi(G) \leq 0\}$, where $\varphi(G)=2 \mathrm{t}_{\hookrightarrow}\left(C_{4} ; G\right)-\mathrm{t}_{\hookrightarrow}\left(K_{2} ; G\right)+\frac{3}{8}$.

Proof. First, note that $\mathrm{t}_{\hookrightarrow}\left(K_{2} ; G\right)=\mathrm{p}^{\mathbf{\bullet}}\left(K_{2} ; G\right)$. Next, we use the fact that

$$
\begin{aligned}
\mathrm{t}_{\hookrightarrow}\left(C_{4} ; G\right) & =\mathrm{t}_{\hookrightarrow}^{\boldsymbol{\Theta}}\left(C_{4} ; G\right)+2 \mathrm{t}_{\hookrightarrow}^{\boldsymbol{\Theta}}\left(D_{4} ; G\right)+\mathrm{t}_{\hookrightarrow}^{\boldsymbol{\Theta}}\left(K_{4} ; G\right) \\
& =\frac{\left|\operatorname{Aut}\left(C_{4}\right)\right|}{4 !} \cdot \mathrm{p}^{\boldsymbol{\Theta}}\left(C_{4} ; G\right)+2 \frac{\left|\operatorname{Aut}\left(D_{4}\right)\right|}{4 !} \cdot \mathrm{p}^{\boldsymbol{\Theta}}\left(D_{4} ; G\right)+\frac{\left|\operatorname{Aut}\left(K_{4}\right)\right|}{4 !} \cdot \mathrm{p}^{\boldsymbol{\Theta}}\left(K_{4} ; G\right) \\
& =\frac{1}{3} \mathrm{p}^{\boldsymbol{\Theta}}\left(C_{4} ; G\right)+\frac{1}{3} \mathrm{p}^{\boldsymbol{\Theta}}\left(D_{4} ; G\right)+\mathrm{p}^{\boldsymbol{\Theta}}\left(K_{4} ; G\right) .
\end{aligned}
$$

Hence,

$$
\begin{aligned}
\varphi(G) & =\frac{2}{3} \mathrm{p}^{\bullet}\left(C_{4} ; G\right)+\frac{2}{3} \mathrm{p}^{\bullet}\left(D_{4} ; G\right)+2 \mathrm{p}^{\bullet}\left(K_{4} ; G\right)-\mathrm{p}^{\bullet}\left(K_{2} ; G\right)+\frac{3}{8} \\
& =\frac{2}{3} \mathrm{p}^{\bullet}\left(C_{4} ; G\right)+\frac{2}{3} \mathrm{p}^{\bullet}\left(D_{4} ; G\right)+2 \mathrm{p}^{\bullet}\left(K_{4} ; G\right)+\mathrm{p}^{\bullet}\left(\overline{K_{2}} ; G\right)-\frac{5}{8} \\
& =\frac{2}{3} \mathrm{p}^{\bullet}\left(C_{4} ; G\right)+\frac{2}{3} \mathrm{p}^{\bullet}\left(D_{4} ; G\right)+2 \mathrm{p}^{\bullet}\left(K_{4} ; G\right)+\sum_{H:|V(H)|=4} \mathrm{p}^{\bullet}\left(\overline{K_{2}} ; H\right) \mathrm{p}^{\bullet}(H ; G)-\frac{5}{8} \\
& =\sum_{H:|V(H)|=4} 2 w_{H} \cdot \mathrm{p}^{\bullet}(H ; G)-\frac{5}{8}
\end{aligned}
$$

Therefore, $\varphi(G) \leq 0$ if and only if $\sum_{H:|V(H)|=4} w_{H} \cdot \mathrm{p}^{\bullet}(H ; G) \leq 5 / 16$ 
An important ingredient in the proof of Theorem 10.9 is the following lemma, which shows that graphs in $\mathcal{D}_{h, w, b}$ are "quasi-random" in the sense that the larger a graph $G=(V, E)$ in $\mathcal{D}_{h, w, b}$ is, the more regular is the pair $\operatorname{Reg}_{\mathbb{a g s}_{0}}(V, V)$.

Lemma 10.12. For every $\delta \in(0,1)$ there is $n_{0}(\delta)$ such that every graph $G \in \mathcal{D}_{h, w, b}$ on $n \geq n_{0}(\gamma)$ vertices satisfies the following. For every $U, V \subseteq V(G)$ with $|U|,|V| \geq \delta n$, we have

$$
\mathrm{e}(U, V)=\left(\frac{1}{2} \pm \delta\right)|U||V|
$$

Proof. We start by showing that for every $\gamma \in(0,1)$ there is $n_{0}(\gamma)$ such that if $G \in \mathcal{D}_{h, w, b}$ is a graph on $n \geq n_{0}(\gamma)$ vertices, then

$$
\mathrm{t}_{\hookrightarrow}\left(K_{2} ; G\right)=\frac{1}{2} \pm \gamma \text { and } \mathrm{t}_{\hookrightarrow}\left(C_{4} ; G\right)=\frac{1}{16} \pm \gamma .
$$

Throughout the proof we can assume, without loss of generality, that $\gamma \leq 14$. Let $\varphi: V\left(C_{4}\right) \rightarrow V(G)$ be a mapping chosen uniformly at random and let $\alpha=1-\frac{(n)_{4}}{n^{4}}$ be the probability that $\varphi$ is non-injective. By the law of total probability, we have

$$
\mathrm{t}\left(C_{4} ; G\right) \leq \mathrm{t}_{\hookrightarrow}\left(C_{4} ; G\right)(1-\alpha)+1 \alpha \leq \mathrm{t}_{\hookrightarrow}\left(C_{4} ; G\right)+\frac{6 n^{3}-11 n^{2}+6 n}{n^{4}} \leq \mathrm{t}_{\hookrightarrow}\left(C_{4} ; G\right)+\frac{12}{n} .
$$

Combine the above inequality with the lower bound of $\mathrm{t}\left(C_{4} ; G\right)$ provided by Lemma 2.15 to get

$$
\mathrm{t}_{\hookrightarrow}\left(C_{4} ; G\right) \geq \mathrm{t}\left(C_{4} ; G\right)-\frac{12}{n} \geq \mathrm{t}\left(K_{2} ; G\right)^{4}-\frac{12}{n} \geq \mathrm{t}_{\hookrightarrow}\left(K_{2} ; G\right)^{4}-\frac{\gamma^{2}}{2},
$$

where the last inequality holds if $n$ is large enough. Now, observe that every $G \in \mathcal{D}_{h, w, b}$ satisfies

$$
2 \mathrm{t}_{\hookrightarrow}\left(K_{2} ; G\right)^{4}-\mathrm{t}_{\hookrightarrow}\left(K_{2} ; G\right)+\frac{3}{8} \leq 2 \mathrm{t}_{\hookrightarrow}\left(C_{4} ; G\right)+\gamma^{2}-\mathrm{t}_{\hookrightarrow}\left(K_{2} ; G\right)+\frac{3}{8}=\varphi(G)+\gamma^{2} \leq \gamma^{2},
$$

where the last inequality follows from Lemma 10.11. Note that the function $x \mapsto 2 x^{4}-x+\frac{3}{8}$ is convex and symmetric around $1 / 2$, where it attains its minimum in $[0,1]$. Therefore, if we had $\mathrm{t}_{\hookrightarrow}\left(K_{2} ; G\right)>\frac{1}{2} \gamma+\gamma$, then we would have

$$
2 \mathrm{t}_{\hookrightarrow}\left(K_{2} ; G\right)^{4}-\mathrm{t}_{\hookrightarrow}\left(K_{2} ; G\right)+\frac{3}{8}>2\left(\frac{1}{2}+\gamma\right)^{4}-\left(\frac{1}{2}+\gamma\right)+\frac{3}{8}=2 \gamma^{4}+4 \gamma^{3}+3 \gamma^{2}>\gamma^{2} .
$$

Similarly, if we had $\mathrm{t}_{\hookrightarrow}\left(K_{2} ; G\right)<\frac{1}{2}-\gamma$, then we would have

$$
2 \mathrm{t}_{\hookrightarrow}\left(K_{2} ; G\right)^{4}-\mathrm{t}_{\hookrightarrow}\left(K_{2} ; G\right)+\frac{3}{8}>2\left(\frac{1}{2}-\gamma\right)^{4}-\left(\frac{1}{2}-\gamma\right)+\frac{3}{8}=2 \gamma^{4}-4 \gamma^{3}+3 \gamma^{2}>\gamma^{2} .
$$

In any case, we see that $\left|\mathrm{t}_{\iota}\left(K_{2} ; G\right)-\frac{1}{2}\right|>\gamma$ would stand in contradiction to ineq. (10.4). Hence, $\mathrm{t}_{\hookrightarrow}\left(K_{2} ; G\right)=\frac{1}{2} \pm \gamma$. By applying Lemma 10.11 again, we get $\mathrm{t}_{\hookrightarrow}\left(C_{4} ; G\right) \leq \frac{1}{16}+\gamma$. By using the intermediate inequality in ineq. (10.3) and $\mathrm{t}_{\varsigma}\left(K_{2} ; G\right) \geq \frac{1}{2}-\gamma$, we get

$$
\mathrm{t}_{\hookrightarrow}\left(C_{4} ; G\right) \geq\left(\frac{1}{2}-\gamma\right)^{4}-\frac{8}{n} \geq \frac{1}{16}-\gamma,
$$

where the last inequality can be easily verified, assuming that $n$ is large enough, after expanding the 
binomial expression. We have thus established ineq. (10.2). A well-known result of Chung, Graham and Wilson [CGW89] states that for every $\delta \in(0,1)$ there is $\gamma=\gamma(\delta)$ such that if a graph $G$ satisfies ineq. (10.2), then for every $U, V \subseteq V(G)$ with $|U|,|V| \geq \delta n$, we have $\mathrm{e}(U, V)=\left(\frac{1}{2} \pm \delta\right)|U||V|$ (see also Lemma 3.8) The lemma follows by combining this result with the above.

In the remainder of this chapter we will show that any graph property $\mathcal{P}$ satisfying the statement of Lemma 10.12 has no 0.1-tester (in particular $\mathcal{D}_{h, w, b}$ is not testable). The general idea is intuitive: suppose by contradiction there was a 0.1-tester for $\mathcal{P}$ which makes $q$ queries. Then, note that any graph $G \in \mathcal{P}$ with $\Omega\left(q^{2}\right)$ vertices and any blow-up of $G$ must have practically the same acceptance probability. In particular, every blow-up $G^{\prime}$ of $G$ must be 0.1-close to $\mathcal{P}$ (for otherwise there would be a gap of $\frac{1}{3}$ between the acceptance probability of $G$ and $\left.G^{\prime}\right)$. On the other hand, if one considers a very large blowup $G^{\prime}$ of $G$, then by Lemma 10.12, graphs of size $\left|V\left(G^{\prime}\right)\right|$ density $\mathrm{d}(A, B)$ close to $\frac{1}{2}$, even for sets $A$ and $B$ whose cardinalities are a $1 /|V(G)|$-fraction of $\left|V\left(G^{\prime}\right)\right|$. But $G^{\prime}$ has, by construction, an equipartition of size $|V(G)|$ with densities either 0 or 1 . In particular, $G^{\prime}$ must be actually far from $\mathcal{P}$.

We proceed to formalize this argument above. For a family of graphs $\mathcal{F}$ and a graph $G$, we define

$$
\mathrm{p}^{\bullet}(\mathcal{F} ; G)=\sum_{F \in \mathcal{F}} \mathrm{p}^{\bullet}(F ; G)
$$

It is well-known that a quasirandom graph has approximately the same distribution of small subgraphs as a random graph with the same density. By combining this with Lemma 10.12, we obtain the following corollary. Note that the expected value of $\mathrm{p}^{\bullet}\left(F ; G\left(n, \frac{1}{2}\right)\right)$ is $2^{-\left(\begin{array}{c}s \\ 2\end{array}\right)} \frac{s !}{|\operatorname{Aut}(F)|}$.

Corollary 10.13. For every $s \geq 2$ and $\delta \in(0,1)$ there is $n_{1}=n_{1}(s, \delta)$ such that every $G \in \mathcal{D}_{h, w, b}$ on $n \geq n_{1}$ vertices satisfies the following. For every family $\mathcal{F}$ of $s$-vertex graphs, we have

$$
\mathrm{p}^{\bullet}(\mathcal{F} ; G)=\sum_{F \in \mathcal{F}} 2^{-\left(\begin{array}{c}
s \\
2
\end{array}\right)} \frac{s !}{|\operatorname{Aut}(F)|} \pm \delta
$$

We are now ready to prove Theorem 10.9 .

Proof of Theorem 10.9. We start by showing that $\mathcal{D}_{h, w, b}$ is non-empty. More specifically, we prove that for every integer $n \geq 4$, there exists an $n$-vertex graph satisfying $\mathcal{D}_{h, w, b}$. Let $G \sim G\left(n, \frac{1}{2}\right)$. We now show that $\mathbb{E}(\varphi(G))=0$, which will imply that there is an $n$-vertex graph with $\varphi(G) \leq 0$, and hence $G \in \mathcal{D}_{h, w, b}$ by Lemma 10.11 . It is easy to see that $\mathbb{E}\left(\mathrm{t}_{\hookrightarrow}\left(K_{2} ; G\right)\right)=\frac{1}{2}$ and $\mathbb{E}\left(\mathrm{t}_{\hookrightarrow}\left(C_{4} ; G\right)\right)=\frac{1}{16}$. Hence

$$
\mathbb{E}(\varphi(G))=2 \mathbb{E}\left(\mathrm{t}_{\hookrightarrow}\left(C_{4} ; G\right)\right)-\mathbb{E}\left(\mathrm{t}_{\hookrightarrow}\left(K_{2} ; G\right)\right)+\frac{3}{8}=0 .
$$

It follows that there is an $n$-vertex graph with $\varphi(G) \leq 0$, and hence $G \in \mathcal{D}_{h, w, b}$ by Lemma 10.11 .

Now suppose by contradiction that $\mathcal{D}_{h, w, b}$ is testable. In particular, there exists a 0.1 -tester for $\mathcal{D}_{h, w, b}$. This implies — by [GT03, Theorem 2] (see also [GT05]) — that there is a canonical 0.1tester $\mathcal{T}$ for $\mathcal{D}_{h, w, b}$. Denote $s$ the sample complexity of $\mathcal{T}$. Then for every $n>0$ there exists a family $\mathcal{F}=\mathcal{F}(n)$ of (rejection) graphs of order $s$ satisfying the following for every $n$-vertex graph $G$.

(i) $\mathrm{p}^{\bullet}(\mathcal{F} ; G) \leq \frac{1}{3}$ if $G \in \mathcal{D}_{h, w, b}$;

(ii) $\mathrm{p}^{\bullet}(\mathcal{F} ; G) \geq \frac{2}{3}$ if $G$ is 0.1 -far from $\mathcal{D}_{h, w, b}$. 
Let $n^{\prime}=\max \left\{9 s^{2}, n_{1}\left(s, \frac{1}{18}\right), 9 s^{2}\right\}$ and $n=n_{0}\left(\frac{1}{n^{\prime}}\right)$, where $n_{1}$ is from Corollary 10.13 and $n_{0}$ is from Lemma 10.12. Let $G^{\prime}$ be an arbitrary $n^{\prime}$-vertex graph which satisfies $\mathcal{D}_{h, w, b}$, and let $G$ be the $\frac{n}{n^{\prime}}$-blow-up of $G^{\prime}$. Denote by $V_{1} \cup \ldots \cup V_{n^{\prime}}=V(G)$ the clusters of this blow-up.

We claim that $G$ is 0.1 -far from $\mathcal{D}_{h, w, b}$. Indeed, fix any $G^{*} \in \mathcal{D}_{h, w, b}$ with $n$ vertices. By our choice of $n$ via Lemma 10.12, and as $\left|V_{i}\right|=\frac{1}{n^{\prime}} \cdot n$, we must have $e_{G^{*}}\left(V_{i}, V_{j}\right)=\left(\frac{1}{2} \pm \frac{1}{n^{\prime}}\right)\left(n / n^{\prime}\right)^{2}$. But since $e_{G}\left(V_{i}, V_{j}\right) \in\left\{0,\left(n / n^{\prime}\right)^{2}\right\}$, we must change at least $\left(\frac{1}{2}-\frac{1}{n^{\prime}}\right)\left(n / n^{\prime}\right)^{2} \geq 0.4\left(n / n^{\prime}\right)^{2}$ edges between $V_{i}$ and $V_{j}$ for every $1 \leq i<j \leq n^{\prime}$, in order to turn $G$ into $G^{*}$. Therefore, the edit distance between $G$ and $G^{*}$ is at least $\left(\begin{array}{c}n^{\prime} \\ 2\end{array}\right) \cdot 0.4\left(n / n^{\prime}\right)^{2} \geq 0.1 n^{2}$, as required.

Now, let $S \in\left(\begin{array}{c}V(G) \\ s\end{array}\right)$ be chosen uniformly at random, and let $\mathcal{B}$ be the event that there exists $1 \leq i \leq n^{\prime}$ for which $\left|S \cap V_{i}\right|>1$. Note that we have $\mathbb{P}(\mathcal{B}) \leq\left(\begin{array}{l}s \\ 2\end{array}\right) / n^{\prime} \leq \frac{1}{9}$, by the choice of $n^{\prime}$. Observe that conditioned on $\mathcal{B}^{c}$, the probability that $S$ is isomorphic to an $s$-vertex $F$ is exactly $\mathrm{p}^{\bullet}\left(F ; G^{\prime}\right)$. Hence, setting $\mathcal{F}=\mathcal{F}(n)$ and $\rho=\sum_{F \in \mathcal{F}} 2^{-\left(\begin{array}{c}s \\ 2\end{array}\right)} \frac{s !}{|\operatorname{Aut}(F)|}$, we have

$$
\begin{aligned}
\mathrm{p}^{\bullet}(\mathcal{F} ; G) & =\mathbb{P}(G[S] \in \mathcal{F}) \leq \mathbb{P}\left(G[S] \in \mathcal{F} \mid B^{c}\right)+\mathbb{P}(B) \\
& \leq \mathbb{P}\left(G[S] \in \mathcal{F} \mid B^{c}\right)+\frac{1}{9} \\
& =\mathrm{p}^{\bullet}\left(\mathcal{F} ; G^{\prime}\right)+\frac{1}{9} \\
& \leq \sum_{F \in \mathcal{F}} 2^{-\left(\begin{array}{c}
s \\
2
\end{array}\right)} \frac{s !}{|\operatorname{Aut}(F)|}+\frac{1}{9}+\frac{1}{9} \\
& =\rho+\frac{2}{9}
\end{aligned}
$$

where in the last inequality we used our choice of $n^{\prime}$ via Corollary 10.13. Fix an arbitrary $n$-vertex graph $G^{*} \in \mathcal{D}_{h, w, b}$. By our choice of $n$ via Corollary 10.13, we have

$$
\mathrm{p}^{\boldsymbol{\Theta}}\left(\mathcal{F} ; G^{*}\right) \geq \sum_{F \in \mathcal{F}} \mathcal{F} 2^{-\left(\begin{array}{c}
s \\
2
\end{array}\right)} \frac{s !}{|\operatorname{Aut}(F)|}-\frac{1}{9}=\rho-\frac{1}{9} .
$$

By combining this with ineq. (10.5), we get $\mathrm{p}^{\boldsymbol{\bullet}}\left(\mathcal{F} ; G^{*}\right) \geq \mathrm{p}^{\boldsymbol{\bullet}}(\mathcal{F} ; G)-\frac{1}{3}$. But this stands in contradiction to $\mathrm{p}^{\boldsymbol{\bullet}}\left(\mathcal{F} ; G^{*}\right)<\frac{1}{3}$ (as $G^{*} \in \mathcal{D}_{h, w, b}$ ) and $\mathrm{p}^{\boldsymbol{\Theta}}(\mathcal{F} ; G) \geq \frac{2}{3}$ (as $G$ is 0.1 -far from $D_{h, w, b}$ ). This completes the proof of the theorem.

\subsection{On the proof of Proposition 3.14 in [GS16]}

Recall that Proposition 3.14 in [GS16] states that there is a tuple $(h, w, b)$ for which $\mathcal{D}_{h, w, b}$ has a POT but $(h, w, b)$ does not have the removal property (see Definition 10.4). Here we argue that the proof of this proposition is incorrect.

The above property $\mathcal{D}_{h, w, b}$ is as follows. Let $\mathcal{R}=\mathcal{R}^{N / 2}$ be the property of all graphs $G$ which are $\frac{|V(G)|}{2}$-regular, and let $\Pi$ be the property of all graphs that are either a union of isolated cliques, or a union of isolated bicliques (i.e. complete bipartite graphs). Then the property $\mathcal{D}_{h, w, b}$ used to prove Proposition 3.14 in [GS16] is $\mathcal{R} \cap \Pi$ (we use this notation to be consistent with [GS16]). It is easy to check that $\mathcal{R} \cap \Pi$ is simply the property of being a balanced biclique (so graphs with an odd number of vertices cannot satisfy $\mathcal{R} \cap \Pi$ ).

First and foremost, the proof of Proposition 3.14 is incorrect because, as we now show, the property $\mathcal{R} \cap \Pi$ cannot at all be represented as $\mathcal{D}_{h, w, b}$. Suppose by contradiction that $\mathcal{R} \cap \Pi=\mathcal{D}_{h, w, b}$ 
for some tuple $(h, w, b)$. In other words, a graph $G$ is a balanced biclique if and only if $z(G):=$ $\sum_{H} w_{H} \cdot \mathrm{p}^{\mathfrak{e}}(H ; G) \leq b$. Let $G$ be a balanced biclique on $2 n$ vertices for $2 n-1 \geq h$. As $z(G)$ is the average of $z\left(G^{\prime}\right)$ over all induced subgraphs $G^{\prime}$ of $G$ on $2 n-1$ vertices, and as $z(G) \leq b$, there must be a graph $G^{\prime}$ on $2 n-1$ vertices satisfying $z\left(G^{\prime}\right) \leq b$. But then $G^{\prime} \in \mathcal{D}_{h, w, b}$, namely $G^{\prime}$ is a balanced biclique, which is impossible as it has an odd number of vertices, a contradiction.

We think that the mistake in the proof of the aforementioned proposition from [GS16] stems from the use of Theorem 2 in [GT03], which transforms a arbitrary tester in a tester into a canonical one. The problem is that the transformation of [GT03, Theorem 2] can disturb the acceptance probability of $n$-vertex graphs by an additive factor of $\Theta(1 / n)$ - such a small perturbation is a consequence of sampling with or without replacement. Such perturbation is not important in neither [GT03, Theorem 2] or [GS16, Theorem 3.12], but it does make a difference in [GS16, Theorem 3.14], where it is critical that these acceptance probabilities are independent of $n$. Due to this perturbation, the representation of the property $\mathcal{R} \cap \Pi$ as $\mathcal{D}_{h, w, b}$ produced by the proof is such that the weights $w_{H}$ depend on $n$, which is not allowed. 


\section{Chapter 11}

\section{Testing hereditary properties of configurations of points in the plane}

Our focus in this chapter is on geometric problems. We will consider problems involving finite point sets in general position - which we call configurations. More specifically, we will study the problem of testing hereditary properties of configurations (that is, properties closed under taking subsets of points). For simplicity, we restrict ourselves to the 2-dimensional case, but it is not difficult to generalize the results here to an arbitrary dimension.

Our perspective complements the approaches in $\left[\mathrm{EKK}^{+} 00, \mathrm{CS01}, \mathrm{CSZ} 00\right]$ - which also study the problem of property testing of configurations of points - in that we use a different notion of distance for the geometric objects in question. Our notion of distance is purely combinatorial in the sense that we work in the category of order types [GP83] (that is, realizable oriented matroids [BLV78] or chirotopes [BS89]; see also [RGZ97, HKSS19]).

A basic problem that can be cast in this framework is that of testing whether a point set $S$ is in convex position, that is, whether every element of $S$ is a vertex of the convex hull $\operatorname{conv}(S)$ of $S$ (that is, an extreme point of $\operatorname{conv}(S)$ ). This problem was investigated in the seminal papers $\left[\mathrm{EKK}^{+} 00\right.$, CS01, CSZ00], with different notions of distance (see also [CFSS17] for a recent study of the high-dimensional case). Our point of view makes it natural to consider the property of being convex as a certain hereditary property of order types, and it also suggests an arguably 'natural' notion of distance.

We present two results on testing general hereditary properties of configurations, Theorems 11.2 and 11.3, and we present a result on a certain extremal function $k_{F}^{*}$ involving configurations, Theorem 11.8, that implies that certain numerical parameters appearing in Theorems 11.2 and 11.3 can be much improved for certain hereditary properties (namely, those defined as the configurations that avoid a fixed 'forbidden' configuration $F$ that satisfies the so-called Erdős-Hajnal property (see Corollary 11.9 in Section 11.3)).

Our general approach is heavily inspired on recent work of Fox and Wei [FW18] addressing property testing problems for permutations under different notions of distance. Another tool that is useful in our context is the regularity lemma for semi-algebraic hypergraphs of Fox, Pach and Suk [FPS16].

This chapter is organized as follows. In Section 11.1, we state Theorem 11.2 Theorem 11.3, after introducing the required definitions. The extremal function $k_{F}^{*}$ mentioned above is introduced in 


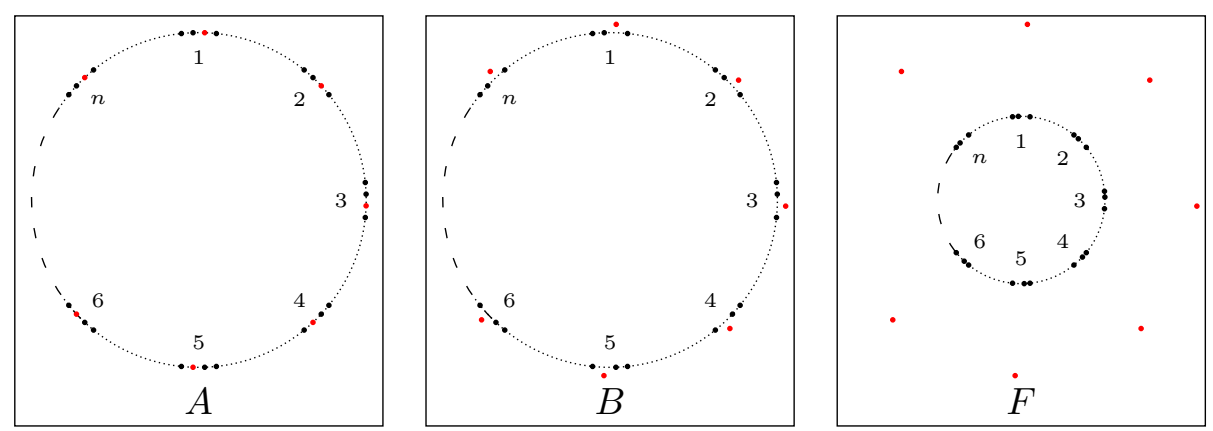

Figure 11.1: Configuration $B$ is a perturbation of a configuration $A$ of 4 n points in convex position.

Section 11.2 and Theorem 11.8 is stated and proved in Section 11.3. Section 11.4 contains the proofs of Theorems 11.2 and 11.3.

\subsection{Formal set-up and main results}

We define a configuration of points as a finite set $C \subseteq \mathbb{R}^{2}$ with no three of them colinear. The orientation $\chi\left(p_{1}, p_{2}, p_{3}\right)$ of a triple of points $\left(p_{1}, p_{2}, p_{3}\right)$ in the plane is given by

$$
\chi\left(p_{1}, p_{2}, p_{3}\right)=\operatorname{sign}\left|\begin{array}{ccc}
x_{1} & x_{2} & x_{3} \\
y_{1} & y_{2} & y_{3} \\
1 & 1 & 1
\end{array}\right|,
$$

where the $x_{i}$ and $y_{i}$ are the coordinates of the $p_{i}(1 \leq i \leq 3)$. Thus $\chi\left(p_{1}, p_{2}, p_{3}\right) \in\{-1,0,1\}$. We say that two configurations $C$ and $C^{\prime} \subseteq \mathbb{R}^{2}$ are isomorphic (denoted by $C \simeq C^{\prime}$ ) or that they have the same order-type if there is a bijection $\iota: C \rightarrow C^{\prime}$ satisfying $\chi(u, v, w)=\chi(\iota(u), \iota(v), \iota(w))$ for all $(u, v, w) \in C^{3}$. We say that a configuration $C$ contains a copy of a configuration $F$ if there is $F^{\prime} \subset C$ such that $F^{\prime}$ is isomorphic to $F$. In such cases, when there is no danger of confusion, we simply write $F \subset C$.

For every (possibly infinite) family $\mathcal{F}$ of configurations, we are interested in the property $\operatorname{Forb}(\mathcal{F})$ $\operatorname{Forb}(\mathcal{F}) \quad$ of all $\mathcal{F}$-free configurations, i.e., all configurations having no copy of members $F \in \mathcal{F}$. If $\mathcal{F}=\{F\}$, we denote the property of all $F$-free configurations simply by $\operatorname{Forb}(F)$.

It is easy to see that every hereditary property $\mathcal{P}$ can be written as $\mathcal{P}=\operatorname{Forb}(\mathcal{F})$ for some $\mathcal{F}$ Conv in fact, one can take $\mathcal{F}$ as the set of all (minimal) configurations not in $\mathcal{P}$. As an example, let Conv be the set of all point configurations in convex position, i.e., the set of all configurations $C=\left\{v_{i}\right\}_{i=1}^{n}$ for which $\chi\left(v_{i}, v_{(i+1) \bmod n}, v\right)=1$ for every $v \notin\left\{v_{i}, v_{(i+1) \bmod n}\right\}$. Then Conv can be written

$N_{4} \quad$ as Conv $=\operatorname{Forb}\left(N_{4}\right)$, where $N_{4}$ is the unique four vertex configuration not in Conv shown in Fig. 11.2.

Czumaj, Sohler and Ziegler [CSZ00] studied property testing of point configurations with respect to the Hamming distance. A configuration $C$ is said to be $\varepsilon$-far in the Hamming distance from a property $\mathcal{P}$ if, for every $P \in \mathcal{P}$, the Hamming distance between $C$ and $P$ is at least $\varepsilon|C|$. In a later work, Czumaj and Sohler [CS01] argued that this notion of distance tells very little about the geometry behind the object. Indeed, although configuration $B$ from Fig. 11.1 is obtained by applying a small perturbation to each of the red points of a convex configuration $A$, it is still $\frac{1}{4}$-far from CONV in the Hamming distance - in particular, $B$ is as far from Conv as the 
configuration $F$ from Fig. 11.1. By taking into account the Euclidian distance between points, the authors of [CS01] considered a distance measure between point configuration that is not sensitive to small perturbations.

Here, we will also consider a distance between point configurations other than the Hamming distance. Unlike [CS01], though, we define a purely combinatorial distance.

We now define our notion of distance, which is natural in this context.

Definition $11.1\left(\mathbf{d}_{1}\right)$. The distance between two point configurations $C$ and $C^{\prime}$ of the same size is defined as

$$
\mathbf{d}_{1}\left(C, C^{\prime}\right)=\min _{\iota} \frac{1}{2 n^{3}} \sum_{(u, v, w) \in C^{3}}|\chi(u, v, w)-\chi(\iota(u), \iota(v), \iota(w))|,
$$

where the minimum is taken over all bijections $\iota: C \rightarrow C^{\prime}$.

Despite the fact that $\mathbf{d}_{1}(\cdot, \cdot)$ is oblivious to the Euclidean distances between the considered points, it is interesting to note that in bears some resemblance to the geometric distance defined by Czumaj and Sohler [CS01] in the sense that $\mathbf{d}_{1}(\cdot, \cdot)$ is not sensitive to "small perturbations" of the point sets being considered. As one would expect, a "small perturbation" in our context is one that flips the orientation of a small number of triples of points. For instance, for $A$ and $B$ as in Fig. 11.1, we have $\mathbf{d}_{1}(B, \mathrm{CONV})=\mathbf{d}_{1}(B, A)=o(1)$, since only triples that intersect more than twice with some group of four points may have its orientation in $A$ different from the one in $B$. As another example, note that the configuration $F$ from Fig. 11.1 is such that $\mathbf{d}_{1}(F, \mathrm{CoNV})=\mathbf{d}_{1}(F, A)=\Omega(1)$.

\subsubsection{Property testing for configurations}

An $\varepsilon$-tester $\mathcal{T}$ for a property $\mathcal{P}$ of configurations is a decision probabilistic algorithm that, when given a configuration $C$ as input, can query the orientation $\chi(u, v, w)$ of triple of points $(u, v, w) \in C^{3}$. Moreover, the $\varepsilon$-tester $\mathcal{T}$ must satisfy the following conditions:

(i) if $C \in \mathcal{P}$, then $\mathbb{P}(\mathcal{T}$ accepts $C) \geq 1-\varepsilon$,

(ii) if $\mathbf{d}_{1}(C, \mathcal{P})>\varepsilon$, then $\mathbb{P}(\mathcal{T}$ rejects $C) \geq 1-\varepsilon$.

If $\mathcal{T}$ accepts inputs $C \in \mathcal{P}$ with probability 1 we say $\mathcal{T}$ has one-sided error. Otherwise, we say $\mathcal{T}$ has two-sided error.

A property $\mathcal{P}$ is testable with query complexity $q:(0,1] \rightarrow \mathbb{N}$ if for every $\varepsilon \in(0,1]$ there is an $\varepsilon$-tester for $\mathcal{P}$ that queries the orientation of at most $q(\varepsilon)$ triples.

The testers considered here will be canonical in the following sense: an $\varepsilon$-tester $\mathcal{T}$ is canonical if, when given an input configuration $C$, the tester samples a set $Q \subseteq C$ of $q=q(\varepsilon)$ points uniformly at random and then accepts $C$ if and only if $Q$ satisfies a certain property AcC. The quantity $q$ is called the sample complexity of $\mathcal{T}$.

\subsubsection{Testing results}

A configuration property $\mathcal{P}$ is hereditary if it is closed under taking subsets. The first result below states that every hereditary property $\mathcal{P}$ is testable (with two-sided error) with sample complexity polynomial on the error parameter $\varepsilon^{-1}$. More than that, this sample complexity is universal, that is, it does not depend on the property $\mathcal{P}$ being tested. However, the testers given by this result need to 

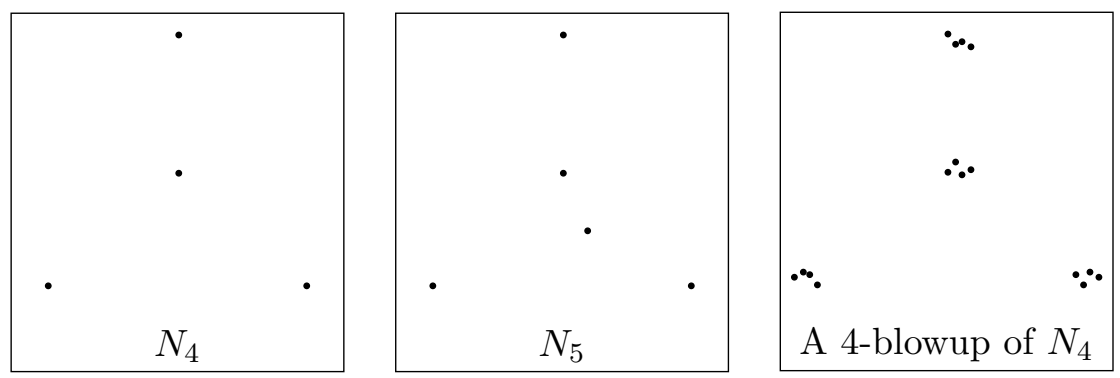

Figure 11.2: The configuration $N_{4}$ and a blow-up

assume that the input configuration is larger than some $n_{0}$, which depends on the property $\mathcal{P}$. More precisely, $n_{0}$ depends on a parameter that we call the blow-up parameter $k_{\mathcal{P}}^{*}$ of $\mathcal{P}$, which is directly inspired in [FW18] (see also the function $\psi_{\mathcal{F}}$ in [AS08b, AFNS09]). The blow-up parameter will be formally defined in Section 11.2. For now, it is enough to keep in mind that $k_{\mathcal{P}}^{*}: \mathbb{N} \backslash\{0\} \rightarrow \mathbb{N}$ is a well-defined function for every hereditary property $\mathcal{P}$.

Theorem 11.2. For every $\varepsilon>0$, there is $q=\operatorname{poly}\left(\varepsilon^{-1}\right)$ such that every hereditary property $\mathcal{P}$ admits a canonical $\varepsilon$-tester with sample complexity $q$ for input configurations of size at least $n_{0}=$ $\operatorname{poly}\left(\varepsilon^{-1}\right) k_{\mathcal{P}}^{*}\left(\operatorname{poly}\left(\varepsilon^{-1}\right)\right)$.

The tester of Theorem 11.2 is such that the property ACC of accepting samples (see Section 11.1.1) is properly contained in $\mathcal{P}$ and, thus, the tester has two-sided error. The next theorem states that every hereditary property can be tested with one sided error.

Theorem 11.3. For every $\varepsilon>0$, every hereditary property $\mathcal{P}$ admits a canonical $\varepsilon$-tester with one-sided error with sample complexity $q^{\prime}=\operatorname{poly}\left(\varepsilon^{-1}\right) k_{\mathcal{P}}^{*}\left(\operatorname{poly}\left(\varepsilon^{-1}\right)\right)$.

\subsection{The blow-up parameter}

We define the concept of blow-ups of configurations, which is analogous to its counterpart in [FW18] for permutations.

Definition 11.4 ( $k$-blow-up). Let $C \subset \mathbb{R}^{2}$ be a configuration and $k$ a positive integer. A configuration $C^{\prime}$ is a $k$-blow-up of $C$ if there is a function $\pi: C^{\prime} \rightarrow C$ satisfying $(i)\left|\pi^{-1}(v)\right|=k$ for every $v \in C$ and $(i i) \chi(x, y, z)=\chi(\pi(x), \pi(y), \pi(z))$ for every $x, y$ and $z \in C^{\prime}$ with $\pi(x), \pi(y)$ and $\pi(z)$ pairwise distinct. The sets $\pi^{-1}(v)$ with $v \in C$ are called the blocks of the blow-up $C^{\prime}$. $\diamond$

In Fig. 11.2 we show a 4-blow-up of the configuration $N_{4}$. We also note that both configurations $A$ and $B$ are 4-blow-ups of the $n$-point convex configuration.

For every hereditary property $\mathcal{P}$, we define the blow-up parameter of $\mathcal{P}$ as the function $k_{\mathcal{P}}^{*}: \mathbb{N} \backslash$ $\{0\} \rightarrow \mathbb{N}$ such that, for every $m>0$, the integer $k_{\mathcal{P}}^{*}(m)$ is the least $k^{*}$ satisfying the following: if a configuration $C$ of size $|C| \leq m$ admits a $k^{*}$-blow-up in $\mathcal{P}$, then $C$ admits an $\ell$-blow-up in $\mathcal{P}$ for every integer $\ell>0$.

Definition $11.5\left(k_{F}^{*}, k_{\mathcal{P}}^{*}\right)$. Let $\mathcal{P}$ be a hereditary property. For every configuration $C$ we let

$k_{\mathcal{P}}^{*}(C)$

$$
k_{\mathcal{P}}^{*}(C)= \begin{cases}\infty, & \text { if } \forall k \exists k \text {-blow-up of } C \text { in } \mathcal{P}, \\ \min \{k: k>0 \text { and } \nexists k \text {-blow-up of } C \text { in } \mathcal{P}\}, & \text { otherwise. }\end{cases}
$$


The blow-up parameter $k_{\mathcal{P}}^{*}: \mathbb{N} \backslash\{0\} \rightarrow \mathbb{N}$ of $\mathcal{P}$ is given by

$$
k_{\mathcal{P}}^{*}(m)= \begin{cases}0, & \text { if } k_{\mathcal{P}}^{*}(C)=\infty \text { for every } C \text { of size at most } m, \\ \max \left\{k_{\mathcal{P}}^{*}(C):|C| \leq m \text { and } k_{\mathcal{P}}^{*}(C)<\infty\right\}, & \text { otherwise }\end{cases}
$$

If $\mathcal{P}=\operatorname{Forb}(F)$ we denote $k_{\mathcal{P}}^{*}$ by $k_{F}^{*}$.

Example 11.6. Let $F=N_{4}$, where $N_{4}$ is as in Fig. 11.2. Then $\operatorname{Forb}\left(N_{4}\right)$ is the set Conv of all convex configurations. We have $k_{F}^{*}(m)=1$ for every $m>0$. Indeed, one can check that, for any $k>0$ and any $C \in \mathrm{Conv}$, it is always possible to construct a convex $k$-blow-up of $C$. Thus $k_{F}^{*}(C)=\infty$ for every $C \in \mathrm{Conv}$. On the other hand, it is trivial that $k_{F}^{*}(C)=1$ for any non-convex $C$.

Example 11.7. Let $F=N_{5}$, where $N_{5}$ is as in Fig. 11.2. Note that $N_{5}$ is isomorphic to any five point configuration in which two of them is contained in the convex hull of the other three. We claim that $k_{F}^{*}(m) \leq 2$ for every $m>0$. Indeed, since Conv $\subset \operatorname{Forb}\left(N_{5}\right)$, we must have $k_{F}^{*}(C)=\infty$ for every $C \in \mathrm{Conv}$, by the reasoning in Example 11.6. On the other hand, if $C \notin \mathrm{CoNv}$, then $C \supset N_{4}$. But since every 2-blow-up of $N_{4}$ contains, in particular, two points inside the convex hull of other three points, every 2-blow-up of $C$ must contain a copy of $N_{5}$. Thus $k_{F}^{*}(C) \leq 2$ for every $C \notin$ Conv.

\subsection{The Erdős-Hajnal property and upper bounds for $k_{F}^{*}$}

For every configuration $F$, let $\mathrm{ES}_{F}(r)$ be the least $n$ such that every configuration $C \in \operatorname{Forb}(F)$ with $|C|=n$ contains $r$ points in convex position. Clearly, $\operatorname{ES}_{F}(r) \leq \operatorname{ES}(r)$, where $\operatorname{ES}(r)$ is the least $n$ such that every $C$ with $|C|=n$ contains $r$ points in convex position. By a well known theorem of Erdös and Szekeres [ES35], we have that $\operatorname{ES}(r)$ is at most exponential in $r$. If $\operatorname{ES}_{F}(r)=$ poly $(r)$, then $F$ is said to satisfy the Erdös-Hajnal property. In [KS06], Károlyi and Solymosi exhibited some configurations that satisfy the Erdös-Hajnal property. We prove the following result on the blow-up parameter $k_{F}^{*}$.

Theorem 11.8. For every configuration $F$ of $f$ points, $k_{F}^{*}(m) \leq \operatorname{EXP}(f \operatorname{poly}(m))$. In addition, if $F$ satisfies the Erdös-Hajnal property, then $k_{F}^{*}(m)=\operatorname{poly}(m, f)$.

Theorem 11.8 gives the following corollary of Theorems 11.2 and 11.3.

Corollary 11.9. Assume the configuration $F$ satisfies the Erdös-Hajnal property and let $\mathcal{P}=$ $\operatorname{Forb}(F)$. For every $\varepsilon>0$, property $\mathcal{P}$ admits an $\varepsilon$-tester with sample complexity $q=\operatorname{poly}\left(\varepsilon^{-1}\right)$ for input configurations of size at least $n_{0}=\operatorname{poly}\left(\varepsilon^{-1}\right) ;$ moreover, $\mathcal{P}$ admits a one-sided canonical $\varepsilon$ tester with sample complexity $q=\operatorname{poly}\left(\varepsilon^{-1}\right)$.

In order to prove Theorem 11.8 we need the following lemma, which states that every large enough blow-up of a configuration is such that we can find 'line segments' $A_{i}$ within each block of the blow-up, where a 'line segment' $A_{i}$ is, roughly speaking, a collection of points in convex position for which every line defined by two of its points divides the plane in the same way.

Lemma 11.10. For any integer $m$ and configuration $F$ with $|F|=f$, there is $k=k_{11.10}(m, F) \leq$ $\operatorname{ES}_{F}(f \operatorname{poly}(m))$ satisfying the following. Let $C$ be a configuration with $|C|=m$ and $C^{\prime}$ be a $k$-blowup of $C$ with blocks $B_{1}, \ldots, B_{m}$. Assume that $C^{\prime} \in \operatorname{Forb}(F)$. Then there are subsets $A_{i} \subset B_{i}(i \in[m])$ such that, for every $i \in[m]$, 
(i) $A_{i}$ is a set $\left\{v_{1}^{(i)}, \ldots, v_{f}^{(i)}\right\}$ of $f$ points in convex position and

(ii) for every $j \in[m] \backslash\{i\}$, there is $\sigma \in\{-1,1\}$ such that $\chi\left(v_{a}^{(i)}, v_{b}^{(i)}, w\right)=\sigma$ for every $1 \leq a<b \leq f$ and every $w \in A_{j}$. In particular, $\bigcup_{i=1}^{m} A_{i}$ is an $f$-blow-up of $C$.

The proof of Lemma 11.10 gives the bound $k_{11.10}(m, F) \leq \operatorname{ES}_{F}(f \operatorname{poly}(m)) \leq \operatorname{EXP}(f \operatorname{poly}(m))$ for general $F$. Moreover, if $F$ satisfies the Erdős-Hajnal property, then one obtains $k_{11.10}(m, F) \leq$ $\operatorname{poly}(m, f)$. Thus, to prove Theorem 11.8 it suffices to prove the following theorem.

Theorem 11.11. For every configuration $F$ of $f$ points, $k_{F}^{*}(m) \leq k_{11.10}(m, F)$.

Proof. Let $k=k_{11.10}(m, F)$. By the definition of $k_{F}^{*}$, we need to show that, for any configuration $C$ with $|C|=m$, either there is no $k$-blow-up of $C$ in $\operatorname{Forb}(F)$ or, for every $\ell>0$, there is an $\ell$-blow-up of $C$ in $\operatorname{Forb}(F)$. If $C \notin \operatorname{Forb}(F)$, then clearly the former holds. We therefore consider the case in which $C \in \operatorname{Forb}(F)$.

Suppose $C \in \operatorname{Forb}(F)$ admits a $k$-blow-up $C^{k}$ with $C^{k} \in \operatorname{Forb}(F)$. Fix an arbitrary $\ell>k$. We show that there is an $\ell$-blow-up $C^{\ell}$ of $C$ with $C^{\ell} \in \operatorname{Forb}(F)$.

We apply Lemma 11.10 with $C^{\prime}=C^{k}$ and obtain sets $A_{1}, \ldots, A_{m} \subset C^{k}$ each of size $f$ satisfying assertions (i) and (ii) from Lemma 11.10. Let $A_{i}=\left\{v_{1}^{(i)}, \ldots, v_{f}^{(i)}\right\}$ for all $i \in[m]$. One can add $\ell-f$ points to each $A_{i}$ in order to obtain sets $L_{1}, \ldots, L_{m}$ such that $(A) L_{i}$ is a set $\left\{u_{1}^{(i)}, \ldots, u_{\ell}^{(i)}\right\}$ of $\ell$ points in convex position, $(B)$ for every $j \in[m] \backslash\{i\}$, there is $\sigma \in\{-1,1\}$ such that $\chi\left(u_{a}^{(i)}, u_{b}^{(i)}, w\right)=\sigma$ for every $1 \leq a<b \leq \ell$ and every $w \in L_{j}$, and $(C)$ the configuration $C^{\ell}:=\bigcup_{i=1}^{m} L_{i}$ is an $\ell$-blow-up of $C$, with blocks $L_{1}, \ldots, L_{m}$.

We claim that $C^{\ell} \in \operatorname{Forb}(F)$. Indeed, suppose by contradiction, suppose that there is a copy $F^{\prime}$ of $F$ in $C^{\ell}$. Let $F^{\prime}=\bigcup_{i=1}^{m}\left\{w_{1}^{(i)}, \ldots, w_{f_{i}}^{(i)}\right\}$, where $\left\{w_{1}^{(i)}, \ldots, w_{f_{i}}^{(i)}\right\} \subseteq L_{i}\left(0 \leq f_{i} \leq f\right)$. We show that $F^{\prime \prime}:=\bigcup_{i=1}^{m}\left\{v_{1}^{(i)}, \ldots, v_{f_{i}}^{(i)}\right\}$ is isomorphic to $F^{\prime}=\bigcup_{i=1}^{m}\left\{w_{1}^{(i)}, \ldots, w_{f_{i}}^{(i)}\right\}$, whence $F \simeq F^{\prime \prime} \subset C^{k} \in$ $\operatorname{Forb}(F)$, which is a contradiction. To prove that $F^{\prime \prime}$ and $F^{\prime}$ are isomorphic, define the bijection $\iota$ by $\iota\left(v_{j}^{(i)}\right)=w_{j}^{(i)}$ for any $i \in[m]$ and $j \in\left[f_{i}\right]$. We now prove that

$$
\chi\left(v_{j_{1}}^{\left(i_{1}\right)}, v_{j_{2}}^{\left(i_{2}\right)}, v_{j_{3}}^{\left(i_{3}\right)}\right)=\chi\left(w_{j_{1}}^{\left(i_{1}\right)}, w_{j_{2}}^{\left(i_{2}\right)}, w_{j_{3}}^{\left(i_{3}\right)}\right) .
$$

First, since both $F^{\prime}$ and $F^{\prime \prime}$ are subsets of blow-ups of $C$, if $i_{1}, i_{2}$ and $i_{3}$ are pairwise distinct, (11.4) holds. Secondly, since for any $i \in[m]$ both $\left\{w_{1}^{(i)}, \ldots, w_{f_{i}}^{(i)}\right\}$ and $\left\{v_{1}^{(i)}, \ldots, v_{f_{i}}^{(i)}\right\}$ are in convex position, we can make (11.4) hold if $i_{1}=i_{2}=i_{3}=i$ by choosing a suitable labeling of the points. Finally, without loss of generality, assume that $i_{1}=i_{2}$ and $i_{2} \neq i_{3}$. In this case (11.4) holds because of $(B)$ above and the fact that $A_{i} \subseteq L_{i}$. The claim is therefore proved. Theorem 11.11 follows.

\subsection{Proofs of Theorem $\mathbf{1 1 . 2}$ and Theorem 11.3}

The main argument used to prove Theorem 11.2 resembles the one used by Fox and Wei [FW18] to show that, under a certain notion of distance, hereditary permutation properties are testable with polynomial (on the error parameter) sample complexity. It also applies the Fox-Pach-Suk Regularity Lemma for uniform semi-algebraic hypergraphs [FPS16] to get a regular partition of configurations of points.

Definition 11.12. We say that a triple $(X, Y, Z) \subset \mathbb{R}^{2} \times \mathbb{R}^{2} \times \mathbb{R}^{2}$ is homogeneous if there is $\sigma \in\{1,-1\}$ such that $\chi(x, y, z)=\sigma$ for every $(x, y, z) \in(X, Y, Z)$. 
A partition $\mathcal{V}=\left\{V_{i}\right\}_{i=1}^{m}$ is called equitable if $\| V_{i}|-| V_{j}|| \leq 1$ for any $i, j \in[m]$.

Lemma 11.13 ([FPS16, Theorem 4.3]). For every $\gamma>0$, there exists $M=M_{11.13}(\gamma)=\operatorname{poly}(1 / \gamma)$ such that every configuration $P,|P|=n \geq 1 / \gamma$, admits an equitable partition $\mathcal{V}=\left\{V_{i}\right\}_{i=1}^{m}$ satisfying the following

(i) $1 / \gamma \leq m \leq M$,

(ii) At most $\gamma n^{3}$ triples of points lie on non-homogeneous triples $\left(V_{i}, V_{j}, V_{\ell}\right)$.

Such a partition is called a $\gamma$-homogeneous partition of $P$.

One consequence of Lemma 11.13 is that (for simplicity, say $n / m \in \mathbb{N}$ ) if one considers a $\gamma$-homogeneous partition $\left\{V_{i}\right\}_{i=1}^{m}$ of a configuration $P$ of $n$ points and an arbitrary set of points $C=\left\{v_{i}\right\}_{i=1}^{m}$ (with $v_{i} \in V_{i}$ ), then $P$ is $\gamma$-close to every $n / m$-blow-up of $C$. Hence, if $P$ is far from a hereditary property $\mathcal{P}$, it follows that every $n / m$-blow-up of $C$ is not in $\mathcal{P}$. Since a large enough sample of $P$ will contain such a set $C$ with high probability, we get the following result.

Lemma 11.14. For every $\varepsilon>0$, the following holds for $M=M_{11.13}(\varepsilon / 2)$ and $q=2 M \log (M / \varepsilon)$ and every hereditary property $\mathcal{P}$ Let $P$ be an order type of size $n \geq M$ and let $Q$ be a set of $q$ points in $P$ chosen uniformly at random. If $\mathbf{d}_{1}(P, \mathcal{P})>\varepsilon$, then, with probability at least $1-\varepsilon$, no $k$-blow-up of $Q$ is in $\mathcal{P}$, where $k=k_{\mathcal{P}}^{*}(M)$.

Proof. Let $\mathcal{V}=\left\{V_{1}, \ldots, V_{m}\right\}$ be an $\varepsilon / 2$-homogeneous partition of $P$. Note that for any $i \in$ $[m], \mathbb{P}\left(Q \cap V_{i}=\emptyset\right) \leq(1-1 /(2 m))^{q} \leq(1-1 /(2 M))^{q}$. Then

$$
\mathbb{P}\left(Q \cap V_{i}=\emptyset \text { for some } i \in[m]\right) \leq M(1-1 /(2 M))^{q} \leq M e^{-q /(2 M)} \leq \varepsilon
$$

Suppose the event $\left[Q \cap V_{i}=\emptyset\right.$ for some $\left.i \in[m]\right]$ does not happen and let $C=\left\{v_{1}, \ldots, v_{m}\right\} \subseteq Q$, where $v_{i} \in V_{i}$ for $i \in[m]$. Write $t:=\lceil n / m\rceil$. Assume that $\mathbf{d}_{1}(P, \mathcal{P})>\varepsilon$, we claim that every $t$ blow-up of $C$ is not in $\mathcal{P}$. Indeed, let $C^{\prime}$ be an arbitrary $t$-blow-up of $C$ and let $\iota: P \rightarrow C^{\prime}$ be an injection that (arbitrarily) maps each point $u \in V_{i}$ to some point $u^{\prime} \in C^{\prime}$ belonging to the $v_{i^{-}}$ block - i.e., some point $u^{\prime} \in \pi^{-1}\left(v_{i}\right)$ where $\pi: C^{\prime} \rightarrow C$ is a function as in Definition 11.4 that witnesses $C^{\prime}$ as an $t$-blow-up of $C$. Let $C_{n}=\iota(P)$ be the image of $\iota$ and thus we can view $\iota: P \rightarrow C_{n}$ as a bijection. For every triple $\left(u_{i}, u_{j}, u_{\ell}\right)$ that belongs to a homogeneous triple $\left(V_{i}, V_{j}, V_{\ell}\right)$ we have $\chi\left(u_{i}, u_{j}, u_{\ell}\right)=\chi\left(v_{i}, v_{j}, v_{\ell}\right)=\chi\left(\iota\left(u_{i}\right), \iota\left(u_{j}\right), \iota\left(u_{\ell}\right)\right)$. Hence, the triples that contribute to the distance between $P$ and $C_{n}$ (see Definition 11.1) are those that belong to non-homogeneous triples. Since $\mathcal{V}$ is an $\varepsilon / 2$-homogeneous partition, it follows that

$$
\mathbf{d}_{1}\left(P, C_{n}\right) \leq \frac{2(\varepsilon / 2) n^{3}}{2 n^{3}}=\varepsilon / 2
$$

Now, since $\mathbf{d}_{1}(P, \mathcal{P})>\varepsilon$, we get that $\mathbf{d}_{1}\left(C_{n}, \mathcal{P}\right)>\varepsilon / 2$, i.e., $C_{n}$ is not in $\mathcal{P}$. Since $\mathcal{P}$ is hereditary, $C^{\prime} \supseteq$ $C_{n}$ is not in $\mathcal{P}$ either. Thus, it follows that no t-blow-up of $C$ is in $\mathcal{P}$. Therefore $k_{\mathcal{P}}^{*}(C)<\infty$. Then, by the definition of $k_{\mathcal{P}}^{*}(M)$ (Definition 11.5), no $k$-blow-up of $C$ is $\mathcal{P}$. Since $C \subseteq Q$ and $\mathcal{P}$ is hereditary, we conclude that no $k$-blow-up of $Q$ is in $\mathcal{P}$.

The previous lemma shows that if $P$ is far from $\mathcal{P}$ then (w.h.p.) a large enough sample $Q$ of $P$ is such that every $k$-blow-up of $Q$ is not in $\mathcal{P}$. Next, we show that if $P \in \mathcal{P}$, then (w.h.p.) a sample $Q$ 
admits a $k$-blow-up in $\mathcal{P}$. This is a consequence of the fact that, given $k$ and $q$, every large enough configuration $P$ contains (w.h.p.) a $k$-blow-up of a sample $Q$ of size $q$. An analogous statement is true when considering other combinatorial objects, like graphs [ES83], permutation [FW18, Lemma $3.2]$, etc.

Lemma 11.15. For every positive integers $q$ and $k$ and $\varepsilon>0$, there exists $n_{0}=M_{11.13}\left(\varepsilon / q^{3}\right) \cdot k$ satisfying the following. If $P \in \mathcal{P},|P| \geq n_{0}$, and $Q$ is a set of $q$ points in $P$ chosen uniformly at random, then, with probability at least $1-\varepsilon$, the configuration $Q$ admits a $k$-blow-up in $\mathcal{P}$.

Proof. Let $\mathcal{W}=\left\{W_{i}\right\}_{i=1}^{m}$ be an $\varepsilon / q^{3}$-homogeneous partition of $P$. The probability that $Q$ contains a triple $\left(w_{i}, w_{j}, w_{\ell}\right) \in W_{i} \times W_{j} \times W_{\ell}$, for some non-homogeneous $\left(W_{i}, W_{j}, W_{\ell}\right)$ is at most

$$
\sum_{W_{i}, W_{j}, W_{\ell}} q^{3} \frac{\left|W_{i}\right|}{n} \frac{\left|W_{j}\right|}{n} \frac{\left|W_{\ell}\right|}{n}=\frac{q^{3}}{n^{3}} \sum_{W_{i}, W_{j}, W_{\ell}}\left|W_{i}\right|\left|W_{j}\right|\left|W_{\ell}\right| \leq \frac{q^{3}}{n^{3}} \cdot \frac{\varepsilon}{q^{3}} n^{3}=\varepsilon .
$$

Hence, with probability at least $1-\varepsilon$ every triple of points of $Q$ lies in a homogeneous triple. Moreover $\left|W_{i}\right| \geq n / m \geq k$. Therefore, one can find a $k$-blowup $Q^{\prime}$ of $Q$ such that $Q^{\prime} \subseteq P$, by arbitrarily picking $k$ vertices from each $W_{i} \in \mathcal{W}$ that satisfies $\left|W_{i} \cap Q\right| \neq \emptyset$. Since $Q^{\prime} \subseteq P$ and $P \in \mathcal{P}$, we must have $Q^{\prime} \in \mathcal{P}$.

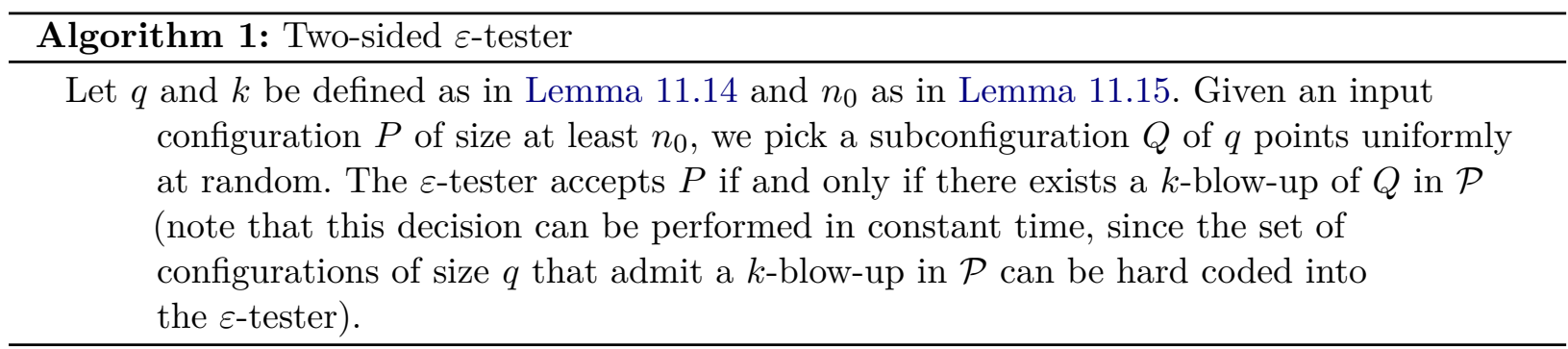

Proof of Theorem 11.2. We use the tester as described in Algorithm 1, whose correctness is guaranteed by Lemma 11.14 and Lemma 11.15 .

Once Theorem 11.2 is proved, Theorem 11.3 follows from a double sampling argument, analogous to the one used in [GT03, Proposition D.2] for graphs.

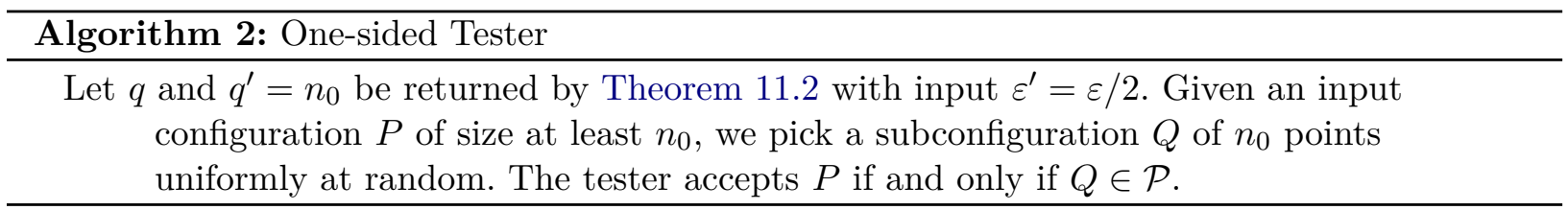

Proof of Theorem 11.3. Let $\mathcal{P}$ be a hereditary configuration property and $\varepsilon \in(0,1]$ be fixed. We use the tester as described in Algorithm 2. Let $q$ and $q^{\prime}=n_{0}$ be returned by Theorem 11.2 with input $\varepsilon^{\prime}=\varepsilon / 2$ and let ACC be the set of configurations $Q$ of size $q$ that makes the tester from Theorem 11.2 accept the input when $Q$ is sampled.

Now we show the correctness of the algorithm. If $P \in \mathcal{P}$, then it is clear that $Q \in \mathcal{P}$. We will show that if $\mathbf{d}_{1}(P, \mathcal{P})>\varepsilon$, then $\mathbb{P}(Q \in \mathcal{P})<\varepsilon$. In fact, let $Q^{\prime}$ be a subconfiguration of $Q$ of size $q$ 
picked uniformly at random. Then

$$
\mathbb{P}\left(Q^{\prime} \in \mathrm{ACC}\right) \geq \mathbb{P}\left(Q^{\prime} \in \mathrm{ACC} \mid Q \in \mathcal{P}\right) \cdot \mathbb{P}(Q \in \mathcal{P}) \geq\left(1-\varepsilon^{\prime}\right) \cdot \mathbb{P}(Q \in \mathcal{P})
$$

since otherwise the $\varepsilon^{\prime}$-tester given by Theorem 11.2 would fail when given $Q$ as input. On the other hand, since $\mathbf{d}_{1}(P, \mathcal{P})>\varepsilon^{\prime}$ and since $Q^{\prime}$ has exactly the same distribution of a subconfiguration of size $q$ picked uniformly at random from $P$, Theorem 11.2 (with input $P$ ) implies $\mathbb{P}\left(Q^{\prime} \in \mathrm{ACC}\right)<\varepsilon^{\prime}$. It follows that

$$
\mathbb{P}(Q \in \mathcal{P})<\frac{\varepsilon^{\prime}}{1-\varepsilon^{\prime}} \leq 2 \varepsilon^{\prime}=\varepsilon
$$

as desired.

\subsection{Discussion}

Theorem 11.8 can be generalized to hereditary properties of the form $\operatorname{Forb}(\mathcal{F})$ with $|\mathcal{F}|$ finite. We hope to address the case in which $\mathcal{F}$ is infinite elsewhere.

\subsection{Proof sketch for Lemma $\mathbf{1 1 . 1 0}$}

We write by $l(x, y)$ as the line defined by two points $x, y$ in the plane. Let $l$ be a line and $B$ be a set of points. Then we say $l$ crosses $B$ if there exist $b_{1}, b_{2} \in B$ such that they are at the different sides of $l$, or, equivalently, the line segment defined by $b_{1}, b_{2}$ intersects $l$. Our key claim is the following.

Claim 11.16. Let $b \in B_{i}$, then any line $l \ni b$ crosses at most one other block $B_{j}$.

Proof. Assume that $l \ni b$ crosses both $B_{j}$ and $B_{j^{\prime}}$. Pick the points $u, u^{\prime} \in B_{j}, v, v^{\prime} \in B_{j^{\prime}}$ such that $u$ and $v$ are at the same side of $l$, and $u^{\prime}, v^{\prime}$ are at the other side of $l$. Consider two lines $l(u, b)$ and $l\left(u^{\prime}, b\right)$. These two lines partition the plane into four regions. By the definition of the blow-up, we know that $\chi(b, u, v)=\chi\left(b, u, v^{\prime}\right)$ and $\chi\left(b, u^{\prime}, v\right)=\chi\left(b, u^{\prime}, v^{\prime}\right)$. Thus, $v$ and $v^{\prime}$ must be in the same region. Moreover, since $v$ and $v^{\prime}$ must be at the different sides of $l$, they must be both in one of the two regions that intersect $l$. Then note that $\chi(b, u, v) \neq \chi\left(b, u^{\prime}, v^{\prime}\right)$ holds, which is a contradiction.

Now we start the sketch of the proof. For any $i \in[m]$, we find a largest subset $C_{i} \subseteq B_{i}$ in convex position. By the definition of $\operatorname{ES}_{F}$, each $C_{i}$ has size at least $f$ poly $(m)$. Let $C_{i}=\left\{v_{1}^{(i)}, \ldots, v_{p}^{(i)}\right\}$ where the points are ordered along the convex polygon under clockwise order. We now color each line segment $v_{j}^{(i)} v_{j+1}^{(i)}$ as follows:

- if $l\left(v_{j}^{(i)}, v_{j+1}^{(i)}\right)$ crosses $C_{p}$ for some $c \in[m], c \neq i$, then give $v_{j}^{(i)} v_{j+1}^{(i)}$ color $p$;

- otherwise, we rotate the line $l\left(v_{j}^{(i)}, v_{j+1}^{(i)}\right)$ around $v_{j+1}^{(i)}$ clock-wise. Give $v_{j}^{(i)} v_{j+1}^{(i)}$ color $p$ if $C_{p}$ is the first block that the line crosses.

\subsection{The Erdős-Hajnal property and upper bounds for $k_{\mathcal{P}}^{*}$}

For every family $\mathcal{F}$ of configurations, let $\operatorname{ES}_{\mathcal{F}}(r)$ be the least $n$ such that every configuration $C \in$ $\operatorname{Forb}(\mathcal{F})$ with $|C|=n$ contains $r$ points in convex position. If $\mathcal{F}=\{F\}$, we denote $\mathrm{ES}_{\mathcal{F}}$ simply 
by $\mathrm{ES}_{F}$. Clearly, $\operatorname{ES}_{\mathcal{F}}(r) \leq \operatorname{ES}(r)$, where $\operatorname{ES}(r)$ is the least $n$ such that every $C$ with $|C|=n$ contains $r$ points in convex position. By a well known theorem of Erdös and Szekeres [ES35], we have that $\operatorname{ES}(r)$ is at most exponential in $r$. If $\operatorname{ES}_{\mathcal{F}}(r)=\operatorname{poly}(r)$, then $\mathcal{F}$ is said to satisfy the Erdös-Hajnal property (if $\mathcal{F}=\{F\}$, then we say that the configuration $F$ has Erdös-Hajnal property). In [KS06], Károyi and Solymosi exhibited some configurations $F$ that satisfy the ErdősHajnal property. Note that if $F$ satisfies the Erdös-Hajnal property, then so does every family $\mathcal{F}$ containing $F$. We prove the following result on the blow-up parameter $k_{\mathcal{P}}^{*}$.

Theorem 11.17. Let $\mathcal{P}=\operatorname{Forb}(\mathcal{F})$ with $\mathcal{F}$ finite. Then $k_{\mathcal{P}}^{*}(m) \leq \operatorname{EXP}(2 f \operatorname{poly}(m))$, where $f=$ $\max _{F \in \mathcal{F}}|F|$. In addition, if $\mathcal{F}$ satisfies the Erdös-Hajnal property, then $k_{\mathcal{P}}^{*}(m)=\operatorname{poly}(m, f)$.

Theorem 11.8 gives the following corollary of Theorems 11.2 and 11.3.

Corollary 11.18. Assume that $\mathcal{F}$ is a finite family satisfying the Erdös-Hajnal property and let $\mathcal{P}=\operatorname{Forb}(\mathcal{F})$. For every $\varepsilon>0, \mathcal{P}$ admits an $\varepsilon$-tester with sample complexity $q=\operatorname{poly}\left(\varepsilon^{-1}\right)$ for input configurations of size at least $n_{0}=\operatorname{poly}\left(\varepsilon^{-1}\right) ;$ moreover, $\mathcal{P}$ admits a one-sided canonical $\varepsilon$ tester with sample complexity $q=\operatorname{poly}\left(\varepsilon^{-1}\right)$.

In order to prove Theorem 11.17 we need the following lemma, which states that every large enough blow-up of a configuration is such that we can find 'line segments' $A_{i}$ within each block of the blow-up, where a 'line segment' $A_{i}$ is, roughly speaking, a collection of points in convex position for which every line defined by two of its points divides the plane in the same way.

Lemma 11.19. For any integers $m$ and $f$, and any (possible infinite)family $\mathcal{F}$, there is $k=$ $k_{11.19}(m, f, \mathcal{F}) \leq \operatorname{ES}_{\mathcal{F}}(f$ poly $(m))$ satisfying the following. Let $C$ be a configuration with $|C|=m$ and $C^{\prime}$ be a k-blow-up of $C$ with blocks $B_{1}, \ldots, B_{m}$. Assume that $C^{\prime} \in \operatorname{Forb}(\mathcal{F})$. Then there are subsets $A_{i} \subset B_{i}(i \in[m])$ such that, for every $i \in[m]$,

(i) $A_{i}$ is a set $\left\{v_{1}^{(i)}, \ldots, v_{f}^{(i)}\right\}$ of $f$ points in convex position and

(ii) for every $j \in[m] \backslash\{i\}$, there is $\sigma \in\{-1,1\}$ such that $\chi\left(v_{a}^{(i)}, v_{b}^{(i)}, w\right)=\sigma$ for every $1 \leq a<b \leq f$ and every $w \in A_{j}$. In particular, $\bigcup_{i=1}^{m} A_{i}$ is an $f$-blow-up of $C$.

The proof of Lemma 11.19 gives the bound $k_{11.19}(m, f, \mathcal{F}) \leq \operatorname{ES}_{\mathcal{F}}(f \operatorname{poly}(m)) \leq \operatorname{EXP}(f \operatorname{poly}(m))$ for general $\mathcal{F}$. Moreover, if $\mathcal{F}$ satisfies the Erdös-Hajnal property, then one obtains $k_{11.19}(m, F) \leq$ poly $(m, f)$. Thus, to prove Theorem 11.17 it suffices to prove the following theorem.

Theorem 11.20. Let $\mathcal{P}=\operatorname{Forb}(\mathcal{F})$, where $\mathcal{F}$ is a finite family of configurations, and $f=$ $\max _{F \in \mathcal{F}}|F|$. Then $k_{\mathcal{P}}^{*}(m) \leq k_{11.19}(m, f, \mathcal{F})$.

Proof. Let $k=k_{11.19}(m, f, \mathcal{F})$. By the definition of $k_{\mathcal{P}}^{*}$, we need to show that, for any configuration $C$ with $|C|=m$, either there is no $k$-blow-up of $C$ in $\operatorname{Forb}(\mathcal{F})$ or, for every $\ell>0$, there is an $\ell$-blow-up of $C$ in $\operatorname{Forb}(\mathcal{F})$. If $C \notin \operatorname{Forb}(\mathcal{F})$, then clearly the former holds. We therefore consider the case in which $C \in \operatorname{Forb}(\mathcal{F})$.

Suppose $C \in \operatorname{Forb}(\mathcal{F})$ admits a $k$-blow-up $C^{k}$ with $C^{k} \in \operatorname{Forb}(\mathcal{F})$. Fix an arbitrary $\ell>k$. We show that there is an $\ell$-blow-up $C^{\ell}$ of $C$ with $C^{\ell} \in \operatorname{Forb}(\mathcal{F})$.

We apply Lemma 11.19 with $C^{\prime}=C^{k}$ and obtain sets $A_{1}, \ldots, A_{m} \subset C^{k}$ each of size $f$ satisfying assertions(i) and (ii) from Lemma 11.19. Let $A_{i}=\left\{v_{1}^{(i)}, \ldots, v_{f}^{(i)}\right\}$ for all $i \in[m]$. One can add $\ell-f$ 
points to each $A_{i}$ in order to obtain sets $L_{1}, \ldots, L_{m}$ such that $(A) L_{i}$ is a set $\left\{u_{1}^{(i)}, \ldots, u_{\ell}^{(i)}\right\}$ of $\ell$ points in convex position, $(B)$ for every $j \in[m] \backslash\{i\}$, there is $\sigma \in\{-1,1\}$ such that $\chi\left(u_{a}^{(i)}, u_{b}^{(i)}, w\right)=\sigma$ for every $1 \leq a<b \leq \ell$ and every $w \in L_{j}$, and $(C)$ the configuration $C^{\ell}:=\bigcup_{i=1}^{m} L_{i}$ is an $\ell$-blow-up of $C$, with blocks $L_{1}, \ldots, L_{m}$.

We claim that $C^{\ell} \in \operatorname{Forb}(\mathcal{F})$. Indeed, suppose by contradiction that there is $F^{\prime} \subseteq C^{\ell}$ isomorphic to some of some member $F \in \operatorname{Forb}(\mathcal{F})$ in $C^{\ell}$. Let $F^{\prime}=\bigcup_{i=1}^{m}\left\{w_{1}^{(i)}, \ldots, w_{f_{i}}^{(i)}\right\}$, where $\left\{w_{1}^{(i)}, \ldots, w_{f_{i}}^{(i)}\right\} \subseteq$ $L_{i}\left(0 \leq f_{i} \leq f\right)$. We show that $F^{\prime \prime}:=\bigcup_{i=1}^{m}\left\{v_{1}^{(i)}, \ldots, v_{f_{i}}^{(i)}\right\}$ is isomorphic to $F^{\prime}=\bigcup_{i=1}^{m}\left\{w_{1}^{(i)}, \ldots, w_{f_{i}}^{(i)}\right\}$, whence $F \simeq F^{\prime \prime} \subset C^{k} \in \operatorname{Forb}(\mathcal{F})$, which is a contradiction. To prove that $F^{\prime \prime}$ and $F^{\prime}$ are isomorphic, define the bijection $\iota$ by $\iota\left(v_{j}^{(i)}\right)=w_{j}^{(i)}$ for any $i \in[m]$ and $j \in\left[f_{i}\right]$. We now prove that

$$
\chi\left(v_{j_{1}}^{\left(i_{1}\right)}, v_{j_{2}}^{\left(i_{2}\right)}, v_{j_{3}}^{\left(i_{3}\right)}\right)=\chi\left(w_{j_{1}}^{\left(i_{1}\right)}, w_{j_{2}}^{\left(i_{2}\right)}, w_{j_{3}}^{\left(i_{3}\right)}\right) .
$$

First, since both $F^{\prime}$ and $F^{\prime \prime}$ are subsets of blow-ups of $C$, if $i_{1}, i_{2}$ and $i_{3}$ are pairwise distinct, (11.4) holds. Secondly, since for any $i \in[m]$ both $\left\{w_{1}^{(i)}, \ldots, w_{f_{i}}^{(i)}\right\}$ and $\left\{v_{1}^{(i)}, \ldots, v_{f_{i}}^{(i)}\right\}$ are in convex position, we can make (11.5) hold if $i_{1}=i_{2}=i_{3}=i$ by choosing a suitable labeling of the points. Finally, without loss of generality, assume that $i_{1}=i_{2}$ and $i_{2} \neq i_{3}$. In this case (11.5) holds because of $(B)$ above and the fact that $A_{i} \subseteq L_{i}$. The claim is therefore proved. Theorem 11.20 follows. 


\section{Bibliography}

$\left[\mathrm{ADL}^{+} 94\right] \quad$ N. Alon, R. A. Duke, H. Lefmann, V. Rödl, and R. Yuster. The algorithmic aspects of the regularity lemma. J. Algorithms, 16(1):80-109, 1994. $\uparrow$ page 29

[AF15] Noga Alon and Jacob Fox. Easily Testable Graph Properties. Combin. Probab. Comput., 24(4):646-657, 2015. $\uparrow$ pages 30, 35, and 73

[AFdlVKK03] Noga Alon, W. Fernandez de la Vega, Ravi Kannan, and Marek Karpinski. Random sampling and approximation of MAX-CSPs. J. Comput. System Sci., 67(2):212-243, 2003. Special issue on STOC2002 (Montreal, QC). $\uparrow$ pages 21, 39, 46, and 47

[AFKS00] Noga Alon, Eldar Fischer, Michael Krivelevich, and Mario Szegedy. Efficient testing of large graphs. Combinatorica, 20(4):451-476, 2000. $\uparrow$ pages 25, 30, 34, 62, and 71

[AFNS09] Noga Alon, Eldar Fischer, Ilan Newman, and Asaf Shapira. A combinatorial characterization of the testable graph properties: it's all about regularity. SIAM J. Comput., 39(1):143-167, 2009. $\uparrow$ pages i, iii, 35, 38, 64, and 94

[AKK99] Sanjeev Arora, David Karger, and Marek Karpinski. Polynomial time approximation schemes for dense instances of NP-hard problems. J. Comput. System Sci., 58(1):193210, 1999. $\uparrow$ page 39

[Alo02] Noga Alon. Testing subgraphs in large graphs. Random Structures Algorithms, 21(3-4):359-370, 2002. Random structures and algorithms (Poznan, 2001). $\uparrow$ pages 29 and 35

[AS06] Noga Alon and Asaf Shapira. A characterization of easily testable induced subgraphs. Comb. Probab. Comput., 15(6):791-805, November 2006. $\uparrow$ pages 30 and 35

[AS08a] Noga Alon and Asaf Shapira. A characterization of the (natural) graph properties testable with one-sided error. SIAM J. Comput., 37(6):1703-1727, 2008. $\uparrow$ pages 30 and 34

[AS08b] Noga Alon and Asaf Shapira. Every monotone graph property is testable. SIAM J. Comput., 38(2):505-522, 2008. $\uparrow$ pages 29, 32, 34, and 94

[AS08c] Noga Alon and Asaf Shapira. A separation theorem in property testing. Combinatorica, 28(3):261-281, 2008. $\uparrow$ page 32

[ASS09] Noga Alon, Asaf Shapira, and Benny Sudakov. Additive approximation for edgedeletion problems. Ann. of Math. (2), 170(1):371-411, 2009. $\uparrow$ pages 36, 62, and 74

[BBS04] József Balogh, Béla Bollobás, and Miklós Simonovits. The number of graphs without forbidden subgraphs. J. Combin. Theory Ser. B, 91(1):1-24, 2004. $\uparrow$ page 61

$\left[\mathrm{BCL}^{+} 08\right]$ Christian Borgs, Jennifer T. Chayes, László Lovász, Vera T. Sós, and Katalin Vesztergombi. Convergent sequences of dense graphs. I. Subgraph frequencies, metric properties and testing. Adv. Math., 219(6):1801-1851, 2008. $\uparrow$ pages i, iii, 1, 21, 28, 35,36 , and 72 
[BLV78] Robert G. Bland and Michel Las Vergnas. Orientability of matroids. J. Combinatorial Theory Ser. B., 24(1):94-123, 1978. $\uparrow$ page 91

[BM15] Rémi Bardenet and Odalric-Ambrym Maillard. Concentration inequalities for sampling without replacement. Bernoulli, 21(3):1361-1385, 2015. $\uparrow$ page 6

[Bol98] Béla Bollobás. Hereditary properties of graphs: asymptotic enumeration, global structure, and colouring. In Proceedings of the International Congress of Mathematicians, Vol. III (Berlin, 1998), pages 333-342, 1998. $\uparrow$ page 61

[BS89] Jürgen Bokowski and Bernd Sturmfels. Computational synthetic geometry, volume 1355 of Lecture Notes in Mathematics. Springer-Verlag, Berlin, 1989. $\uparrow$ page 91

[BS11] József Balogh and Wojciech Samotij. The number of $K_{m, m}$-free graphs. Combinatorica, 31(2):131-150, 2011. 个 page 61

[BT97] Dimitris Bertsimas and John N. Tsitsiklis. Introduction to linear organisation, volume 6 of Athena scientific optimization and computation series. Athena Scientific, 1997. $\uparrow$ pages 40 and 41

[CF12] David Conlon and Jacob Fox. Bounds for graph regularity and removal lemmas. Geom. Funct. Anal., 22(5):1191-1256, 2012. $\uparrow$ pages 23, 24, 25, 29, 30, and 35

[CFSS17] Xi Chen, Adam Freilich, Rocco A. Servedio, and Timothy Sun. Sample-based highdimensional convexity testing. In Approximation, randomization, and combinatorial optimization. Algorithms and techniques, volume 81 of LIPIcs. Leibniz Int. Proc. Inform., pages Art. No. 37, 20. Schloss Dagstuhl. Leibniz-Zent. Inform., Wadern, 2017. $\uparrow$ page 91

[CGW89] F. R. K. Chung, R. L. Graham, and R. M. Wilson. Quasi-random graphs. Combinatorica, 9(4):345-362, 1989. $\uparrow$ pages 83 and 87

[CS01] Artur Czumaj and Christian Sohler. Property testing with geometric queries (extended abstract). In Algorithms-ESA 2001 (Arhus), volume 2161 of Lecture Notes in Comput. Sci., pages 266-277. Springer, Berlin, 2001. $\uparrow$ pages 91, 92, and 93

[CSZ00] Artur Czumaj, Christian Sohler, and Martin Ziegler. Property testing in computational geometry (extended abstract). In Algorithms-ESA 2000 (Saarbrücken), volume 1879 of Lecture Notes in Comput. Sci., pages 155-166. Springer, Berlin, 2000. $\uparrow$ pages 91 and 92

[dJdV19] Rémi de Joannis de Verclos. Chordal graphs are easily testable, 2019. Avaiable at arxiv:1902.06135v1. $\uparrow$ pages 35 and 73

[DLR95] Richard A. Duke, Hanno Lefmann, and Vojtěch Rödl. A fast approximation algorithm for computing the frequencies of subgraphs in a given graph. SIAM J. Comput., 24(3):598-620, 1995. $\uparrow$ page 61

[dlV96] W. Fernandez de la Vega. MAX-CUT has a randomized approximation scheme in dense graphs. Random Structures Algorithms, 8(3):187-198, 1996. $\uparrow$ page 39

[EFR86] Paul Erdős, Péter Frankl, and Vojtěch Rödl. The asymptotic number of graphs not containing a fixed subgraph and a problem for hypergraphs having no exponent. Graphs Combin., 2(2):113-121, 1986. $\uparrow$ pages 61 and 70

[EKK $\left.{ }^{+} 00\right] \quad$ Funda Ergün, Sampath Kannan, S. Ravi Kumar, Ronitt Rubinfeld, and Mahesh Viswanathan. Spot-checkers. J. Comput. System Sci., 60(3):717-751, 2000. 30th Annual ACM Symposium on Theory of Computing (Dallas, TX, 1998). $\uparrow$ page 91 
[EKR76] Paul Erdős, Daniel J. Kleitman, and Bruce L. Rothschild. Asymptotic enumeration of $K_{n}$-free graphs. In Colloquio Internazionale sulle Teorie Combinatorie (Rome, 1973), Tomo II, pages 19-27. Atti dei Convegni Lincei, No. 17. Accad. Naz. Lincei, Rome, 1976. $\uparrow$ page 61

[ES35] P. Erdős and G. Szekeres. A combinatorial problem in geometry. Compositio Math., 2:463-470, 1935. $\uparrow$ pages 95 and 100

[ES83] Paul Erdős and Miklós Simonovits. Supersaturated graphs and hypergraphs. Combinatorica, 3(2):181-192, 1983. $\uparrow$ pages 65 and 98

[FK99] Alan Frieze and Ravi Kannan. Quick approximation to matrices and applications. Combinatorica, 19(2):175-220, 1999. $\uparrow$ pages 22, 23, and 38

[FLZ17] Jacob Fox, László Miklós Lovász, and Yufei Zhao. On regularity lemmas and their algorithmic applications. Combin. Probab. Comput., 26(4):481-505, 2017. $\uparrow$ page 61

[FMS10] Eldar Fischer, Arie Matsliah, and Asaf Shapira. Approximate hypergraph partitioning and applications. SIAM J. Comput., 39(7):3155-3185, 2010. $\uparrow$ page 51

[FN07] Eldar Fischer and Ilan Newman. Testing versus estimation of graph properties. SIAM J. Comput., 37(2):482-501, 2007. $\uparrow$ pages i, iii, 1, 2, 35, 36, 62, and 74

[Fox11] Jacob Fox. A new proof of the graph removal lemma. Ann. of Math. (2), 174(1):561579,2011 . $\uparrow$ pages 29,35 , and 62

[FPS16] Jacob Fox, János Pach, and Andrew Suk. A polynomial regularity lemma for semialgebraic hypergraphs and its applications in geometry and property testing. SIAM J. Comput., 45(6):2199-2223, 2016. $\uparrow$ pages 91, 96, and 97

[FR20] Nimrod Fiat and Dana Ron. On efficient distance approximation for graph properties. CoRR, abs/2001.01452, 2020. $\uparrow$ pages 36, 39, and 76

[Für95] Zoltán Füredi. Extremal hypergraphs and combinatorial geometry. In Proceedings of the International Congress of Mathematicians, Vol. 1, 2 (Zürich, 1994), pages 1343-1352. Birkhäuser, Basel, 1995. ^ page 29

[FW18] Jacob Fox and Fan Wei. Fast property testing and metrics for permutations. Combin. Probab. Comput., 27(4):539-579, 2018. $\uparrow$ pages 91, 94, 96, and 98

[GGR98] Oded Goldreich, Shafi Goldwasser, and Dana Ron. Property testing and its connection to learning and approximation. J. ACM, 45(4):653-750, 1998. $\uparrow$ pages i, iii, 2, 29, $34,35,37,39,49$, and 51

[Gol11a] Oded Goldreich. A brief introduction to property testing. In Studies in complexity and cryptography, volume 6650 of Lecture Notes in Comput. Sci., pages 465-469. Springer, Heidelberg, 2011. $\uparrow$ page 34

[Gol11b] Oded Goldreich. Contemplations on testing graph properties. In Studies in complexity and cryptography, volume 6650 of Lecture Notes in Comput. Sci., pages 547-554. Springer, Heidelberg, 2011. $\uparrow$ page 30

[Gow97] W. T. Gowers. Lower bounds of tower type for Szemerédi's uniformity lemma. Geom. Funct. Anal., 7(2):322-337, 1997. $\uparrow$ pages 25 and 62

[GP83] Jacob E. Goodman and Richard Pollack. Multidimensional sorting. SIAM J. Comput., 12(3):484-507, 1983. $\uparrow$ page 91 
[GR11] Oded Goldreich and Dana Ron. On proximity-oblivious testing. SIAM J. Comput., 40(2):534-566, 2011. $\uparrow$ page 82

[GS16] Oded Goldreich and Igor Shinkar. Two-sided error proximity oblivious testing. Random Structures Algorithms, 48(2):341-383, 2016. $\uparrow$ pages i, iii, vi, 2, 73, 78, 82, $83,85,88$, and 89

[GS17] Lior Gishboliner and Asaf Shapira. Removal lemmas with polynomial bounds. In STOC'17-Proceedings of the 49th Annual ACM SIGACT Symposium on Theory of Computing, pages 510-522. ACM, New York, 2017. $\uparrow$ page 35

[GS19] Lior Gishboliner and Asaf Shapira. Efficient removal without efficient regularity. Combinatorica, 39(3):639-658, 2019. $\uparrow$ pages 30 and 35

[GSS20a] Lior Gishboliner, Asaf Shapira, and Henrique Stagni. Testing linear inequalities of subgraph statistics. In Thomas Vidick, editor, 11th Innovations in Theoretical Computer Science Conference, ITCS 2020, January 12-14, 2020, Seattle, Washington, USA, volume 151 of LIPIcs, pages 43:1-43:9. Schloss Dagstuhl - Leibniz-Zentrum für Informatik, 2020. $\uparrow$ page 3

[GSS20b] Lior Gishboliner, Asaf Shapira, and Henrique Stagni. Testing linear inequalities of subgraph statistics. Electron. Colloquium Comput. Complex., 27:107, 2020. $\uparrow$ page 3

[GT03] Oded Goldreich and Luca Trevisan. Three theorems regarding testing graph properties. Random Structures Algorithms, 23(1):23-57, 2003. $\uparrow$ pages 33, 34, 57, 84, 87, 89 , and 98

[GT05] Oded Goldreich and Luca Trevisan. Errata to the paper: Three theorems regarding testing graph properties. http://www.wisdom.weizmann.ac.il/ oded/PS/tt-err.ps, 2005. $\uparrow$ pages 33 and 87

[Hat10] Hamed Hatami. Graph norms and Sidorenko's conjecture. Israel J. Math., 175:125150, 2010. $\uparrow$ page 14

$\left[\mathrm{HKL}^{+}\right] \quad$ C. Hoppen, Y. Kohayakawa, R. Lang, H. Lefmann, and H. Stagni. On the query complexity of estimating the distance to hereditary graph properties. submitted. $\uparrow$ pages 3 and 36

$\left[\mathrm{HKL}^{+} 16\right]$ Carlos Hoppen, Yoshiharu Kohayakawa, Richard Lang, Hanno Lefmann, and Henrique Stagni. Estimating parameters associated with monotone properties. In APPROX/RANDOM 2016: Approximation, Randomization, and Combinatorial Optimization. Algorithms and Techniques, volume 60 of LIPIcs. Leibniz Int. Proc. Inform., pages 35:1-35:13. Schloss Dagstuhl. Leibniz-Zent. Inform., Wadern, 2016. $\uparrow$ pages 3 and 36

$\left[\mathrm{HKL}^{+} 17\right] \quad$ Carlos Hoppen, Yoshiharu Kohayakawa, Richard Lang, Hanno Lefmann, and Henrique Stagni. Estimating the distance to a hereditary graph property. Electronic Notes in Discrete Mathematics, 61:607-613, 2017. The European Conference on Combinatorics, Graph Theory and Applications (EUROCOMB '17). $\uparrow$ pages 3 and 36

$\left[\mathrm{HKL}^{+} 20\right] \quad$ C. Hoppen, Y. Kohayakawa, R. Lang, H. Lefmann, and H. Stagni. Estimating parameters associated with monotone properties. Combin. Probab. Comput., 29(4):616-632, 2020. $\uparrow$ pages 3,36 , and 58

[HKSS18] Jie Han, Yoshiharu Kohayakawa, Marcelo Tadeu Sales, and Henrique Stagni. Property testing for point sets on the plane. In LATIN 2018: Theoretical informatics, volume 10807 of Lecture Notes in Comput. Sci., pages 584-596. Springer, Cham, 2018. $\uparrow$ page 3 
[HKSS19] Jie Han, Yoshiharu Kohayakawa, Marcelo T. Sales, and Henrique Stagni. Extremal and probabilistic results for order types. In Proceedings of the Thirtieth Annual ACM-SIAM Symposium on Discrete Algorithms, pages 426-435. SIAM, Philadelphia, $\mathrm{PA}, 2019 . \uparrow$ page 91

[Hoe63] Wassily Hoeffding. Probability inequalities for sums of bounded random variables. J. Amer. Statist. Assoc., 58:13-30, 1963. $\uparrow$ pages 5 and 6

[Juk01] Stasys Jukna. Extremal combinatorics. Texts in Theoretical Computer Science. An EATCS Series. Springer-Verlag, Berlin, 2001. With applications in computer science. $\uparrow$ page 67

[KS06] Gyula Károlyi and József Solymosi. Erdős-Szekeres theorem with forbidden order types. J. Combin. Theory Ser. A, 113(3):455-465, 2006. $\uparrow$ pages 95 and 100

[Lon66] David London. Two inequalities in nonnegative symmetric matrices. Pacific J. Math., 16:515-536, 1966. $\uparrow$ page 13

[Lov12] László Lovász. Large networks and graph limits, volume 60 of American Mathematical Society Colloquium Publications. American Mathematical Society, Providence, RI, 2012. $\uparrow$ pages 10,26 , and 28

[LS07] László Lovász and Balázs Szegedy. Szemerédi's lemma for the analyst. Geom. Funct. Anal., 17(1):252-270, 2007. $\uparrow$ page 23

[McD89] Colin McDiarmid. On the method of bounded differences. In Surveys in combinatorics, 1989 (Norwich, 1989), volume 141 of London Math. Soc. Lecture Note Ser., pages 148-188. Cambridge Univ. Press, Cambridge, 1989. $\uparrow$ page 6

[MS08] Claire Mathieu and Warren Schudy. Yet another algorithm for dense max cut: go greedy. In Proceedings of the Nineteenth Annual ACM-SIAM Symposium on Discrete Algorithms, pages 176-182. ACM, New York, 2008. $\uparrow$ page 39

[MS19] Guy Moshkovitz and Asaf Shapira. A sparse regular approximation lemma. Trans. Amer. Math. Soc., 371(10):6779-6814, 2019. ^ page 29

[PRR06] Michal Parnas, Dana Ron, and Ronitt Rubinfeld. Tolerant property testing and distance approximation. J. Comput. System Sci., 72(6):1012-1042, 2006. $\uparrow$ page 35

[PS92] H. J. Prömel and A. Steger. The asymptotic number of graphs not containing a fixed color-critical subgraph. Combinatorica, 12(4):463-473, 1992. $\uparrow$ page 61

[PS96] Hans Jürgen Prömel and Angelika Steger. Counting $H$-free graphs. Discrete Math., 154(1-3):311-315, 1996. $\uparrow$ page 61

[RD85] V. Rödl and R. A. Duke. On graphs with small subgraphs of large chromatic number. Graphs Combin., 1(1):91-96, 1985. $\uparrow$ page 35

[RGZ97] Jürgen Richter-Gebert and Günter M. Ziegler. Oriented matroids. In Handbook of discrete and computational geometry, CRC Press Ser. Discrete Math. Appl., pages 111-132. CRC, Boca Raton, FL, 1997. ^ page 91

[RS78] I. Z. Ruzsa and E. Szemerédi. Triple systems with no six points carrying three triangles. In Combinatorics (Proc. Fifth Hungarian Colloq., Keszthely, 1976), Vol. II, volume 18 of Colloq. Math. Soc. János Bolyai, pages 939-945. North-Holland, Amsterdam-New York, 1978. $\uparrow$ page 29

[RV07] Mark Rudelson and Roman Vershynin. Sampling from large matrices: an approach through geometric functional analysis. J. ACM, 54(4):Art. 21, 19, 2007. $\uparrow$ page 21 
[Sid91] A. F. Sidorenko. Inequalities for functionals generated by bipartite graphs. Diskret. Mat., 3(3):50-65, 1991. $\uparrow$ page 13

[Sid93] Alexander Sidorenko. A correlation inequality for bipartite graphs. Graphs Combin., 9(2):201-204, 1993. $\uparrow$ page 13

[SS20] Asaf Shapira and Henrique Stagni. Partitions of a sample and applications. 2020. In preparation. $\uparrow$ page 3

[Sze78] Endre Szemerédi. Regular partitions of graphs. In Problèmes combinatoires et théorie des graphes (Colloq. Internat. CNRS, Univ. Orsay, Orsay, 1976), volume 260 of Colloq. Internat. CNRS, pages 399-401. CNRS, Paris, 1978. $\uparrow$ pages i, 24, 62, and 64 


\section{Symbol Index}

\begin{tabular}{|c|c|}
\hline, \pm 5 & $\mathrm{e}(G):$ for graph, 7 \\
\hline$\leq:$ for graphs, 7 & $\operatorname{ex}(\Gamma, \mathcal{F}), 70$ \\
\hline$G \leq \Gamma, 7$ & $\overline{\operatorname{ex}}(\Gamma, \mathcal{F}), 70$ \\
\hline$R \leq R^{\prime}, 7$ & $\operatorname{Forb}(\mathcal{F})$ : for graphs, 9 \\
\hline $\mathbb{Z}_{0}^{0}, 8$ & $\operatorname{Forb}(\mathcal{F})$ : for points, 92 \\
\hline$\|G\|_{1}, 9$ & $\operatorname{Forb}(F), 9$ \\
\hline$\|G\|_{2}, 9$ & Forb $_{>}(\mathcal{F}), 63$ \\
\hline$\|G\|_{\square}, 10$ & Forb $_{>}(R, \mathcal{F}), 63$ \\
\hline$\|G\|_{\triangle}, 10$ & $\operatorname{Forb}^{\bullet}(\mathcal{F}), 9$ \\
\hline$\|G\|_{\square_{f, g}^{+}}, 10$ & $\operatorname{Forb}^{\bullet}(F), 9$ \\
\hline$\|G\|_{\square_{f, g}}, 10$ & $F \rightarrow G, 11$ \\
\hline$\oplus, 17$ & $G / \mathcal{V}, 18$ \\
\hline $\mathcal{V} \oplus S, 17$ & $G_{\mathcal{V}}, 17$ \\
\hline$\preceq, 17$ & $G[X, Y], 8$ \\
\hline $\bar{S}, 5$ & $\mathcal{G}, 7$ \\
\hline $\mathrm{ACC} \mathrm{ACC}_{n}, 32$ & $\mathcal{G}_{Z}(V), 6$ \\
\hline$f$-attestable, 75 & $\mathcal{G}(V), 7$ \\
\hline $\operatorname{Aut}(G), 8$ & $\mathcal{G}^{*}(V), 7$ \\
\hline $\operatorname{aut}(G), 8$ & $G \leq \Gamma, 7$ \\
\hline$C_{4}, 8$ & $G[Q], 7$ \\
\hline$\chi\left(p_{1}, p_{2}, p_{3}\right), 92$ & $G(X, Y): G$ graph, 7 \\
\hline Conv, 92 & $H=(X, Y, E), 8$ \\
\hline $\mathcal{D}_{h, w, b}, 82$ & $\mathcal{H}_{Z}(X, Y), 8$ \\
\hline $\mathrm{d}_{G}(X, Y), 7$ & $\mathcal{H}(X, Y), 8$ \\
\hline $\mathrm{d}(X, Y), 7$ & $h_{\mathcal{F}}(\Gamma), 61$ \\
\hline $\mathrm{d}(H)$ : for bipartite graph, 8 & $\operatorname{Hom}(F, G), 12$ \\
\hline $\mathbf{d}_{1}\left(C, C^{\prime}\right)$ : for configurations, 93 & $\operatorname{hw}_{\varphi}(F ; G), 11$ \\
\hline $\mathbf{d}_{1}(G, H):$ for graphs, 9 & $\operatorname{hw}_{\varphi, \psi}(F ; H), 12$ \\
\hline $\mathbf{d}_{\mathcal{P}}, 34$ & $\mathrm{hw}_{\varphi, \psi}^{\mathbf{e}}(F ; H), 12$ \\
\hline $\mathbf{d}_{\mathcal{P}}^{(K, \varepsilon)}(\Gamma), 75$ & $\operatorname{hw}_{\varphi}^{e}(F ; G), 11$ \\
\hline$E(G), 7$ & $H(X, Y):$ for bipartite graph, 8 \\
\hline$E(H), \mathrm{e}(H)$ : for bipartite graph, 8 & $k_{\mathcal{P}}^{*}(C), 94$ \\
\hline $\mathrm{ES}_{\mathcal{F}}(r), 99$ & $k_{\mathcal{P}}^{*}(m), 95$ \\
\hline $\mathrm{ES}_{F}(r), 95$ & $d K_{X, Y}, 23$ \\
\hline $\mathrm{e}(A, B):$ for graph, 7 & $d K_{S}, 23$ \\
\hline
\end{tabular}


$N_{4}, 92$

$\mathrm{p}(F ; G), 12$

$\mathrm{p}^{\mathbf{e}}(F ; G), 12$

$\wp_{K}, \wp_{K}^{\leq}, 18$

$\Pi_{k, \bar{d}}, 51$

$\Pi_{k, \bar{d}, \bar{\gamma}}, 51$

$\Pi_{R}, 74$

$f$-recoverable, 64

$\operatorname{Reg}_{\mathscr{s}_{0}}(X, Y), 19$

$R \leq R^{\prime}, 7$

$\mathrm{t}(F ; G), 11$

$\mathrm{t}_{\hookrightarrow}(F ; G), 11$

$\ddot{\mathrm{t}}_{F}(A, B), 13$

$\ddot{\mathrm{t}}_{F}(H), 13$

$\mathrm{t}^{\bullet}(F ; G), 12$

$\mathrm{t}_{\hookrightarrow}^{\mathbf{O}}(F ; G), 12$

$\mathcal{V} \oplus S, 17$

$V(G), 7$ 